Dissertation zur Erlangung des Doktorgrades der Fakultät für Chemie und Pharmazie

der Ludwig-Maximilians-Universität München

\title{
Structure and Function of Hip, an Attenuator of the Hsp70 Chaperone Cycle
}

\section{Zhuo Li}

aus Dalian, Liaoning, China

München, 2013 


\section{$\underline{\text { Erklärung }}$}

Diese Dissertation wurde im Sinne von $\S 7$ der Promotionsordnung von 28. November 2011 von Herrn Prof. Dr. F. Ulrich Hartl betreut.

\section{Eidesstattliche Versicherung}

Diese Dissertation wurde selbständig, ohne unerlaubte Hilfsmittel erarbeitet.

München, den 03.12.2013

Dissertation eingereicht am: 03.12.2013

1. Gutachter

Prof. Dr. F. Ulrich Hartl

2. Gutachter:

Prof. Dr. Roland Beckmann

Mündliche Prüfung am: 13.02.2014 


\section{Acknowledgement}

I am particularly thankful to Prof. Dr. F. Ulrich Hartl for giving me the opportunity to work in his international and interdisciplinary department at Max Planck Institute of Biochemistry. I am grateful to Dr. Manajit Hayer-Hartl for her support and advice.

I especially express my deepest gratitude to my supervisor Dr. Andreas Bracher. It has been my pleasure and my luck to work with him, a knowledgeable, efficient, conscientious and distinguished scientist, who gave me many valuable and critical suggestions and managed many details throughout my entire $\mathrm{PhD}$ study. He patiently and meticulously guided me and encouraged me to undertake and keep our project going on schedule. I am especially thankful for his constant help and suggestions in my thesis corrections.

I would like to extend my gratitude to Dr. Swasti Raychaudhuri for his crucial contributions to this project. I am also deeply grateful to Dr. Mathias Stotz for his help and discussion during the various phases of my project. Furthermore, many great thanks to Dr. Qiaoyun Shi, Dr. Guoxin Jiang, Ayano Konagai, Dr. Yujin Kim, Dr. Joshi Jidnyasa, Dr. Young-Jun Choe, Dr. Niti Kumar, Sneha Kumar, Dr. Frédéric Frottin, Dr. Rajat Gupta and Dr. Taotao Chen. I extend my sincerest appreciation and thanks to my office members Dr. Sigrun Polier, Leonie Mönkemeyer, Tobias Neudegger, Dr. Cuimin Liu, Dr. Oliver Müller-Cajar, Dr. Candace Tsai, Dr. Amanda Windhof, Dr. Paulo Durao and Thomas Hauser. I am thankful to Evelyn Frey-Royston, Silke Leuze-Bütün, Andrea Obermayr-Rauter and Emmanuel Burghardt, Albert Ries as well as Dr. Stefan Pinkert for their invaluable supports. To the rest of the department, I really appreciated the open-minded scientific atmosphere and enjoyable working environment.

I want to thank all the support from Karina Valer and Sabine Pleyer for initial screening of crystallization conditions in MPIB Crystallization Facility. I am especially grateful to Dr. Debora Makino for collecting diffraction data and to Dr. Claire Basquin for her help with SAXS data analysis.

I am thankful for my thesis advisory board members Prof. Dr. Elena Conti and Dr. Roman Körner.

I also want to thank for the members of my Ph.D committee Prof. Dr. Roland Beckmann, Prof. Dr. Elena Conti, Prof. Dr. Jörg Nickelsen, Prof. Dr. Don Lamb and Dr. Petra Wendler for critical evaluation of this thesis.

I am grateful for my good friends Zijing Gong, Dr. Weihua Qin and Xixi Jia. I am really appreciated all the fun times we have shared and all the support they gave me.

Finally, I give a very special note of gratitude to my parents Xiuling Shi and Shijie Li, who have supported and encouraged me over the years. 


\section{Contents}

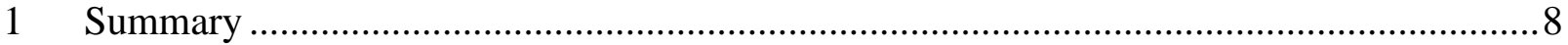

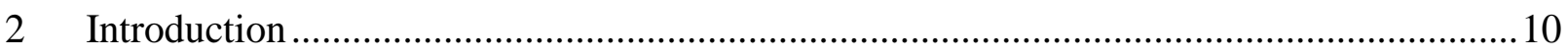

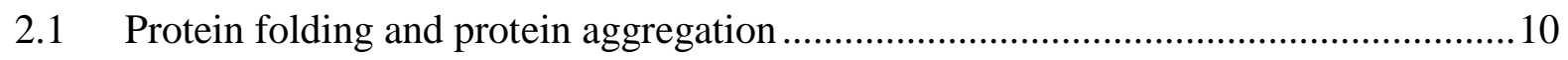

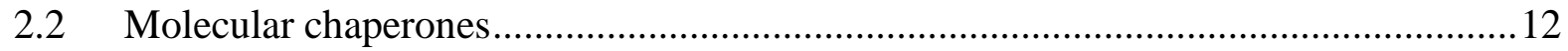

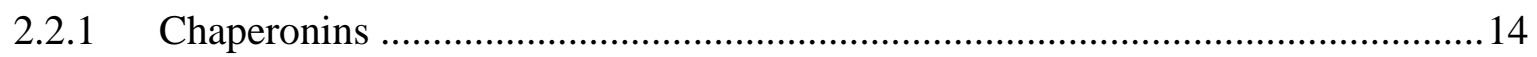

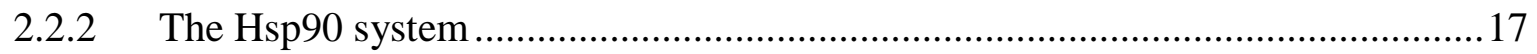

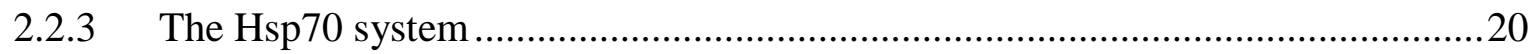

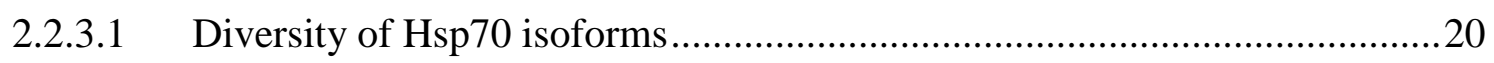

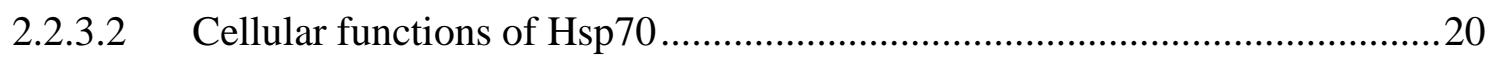

2.2.3.3 Structure and working cycle of Hsp70 .....................................................2

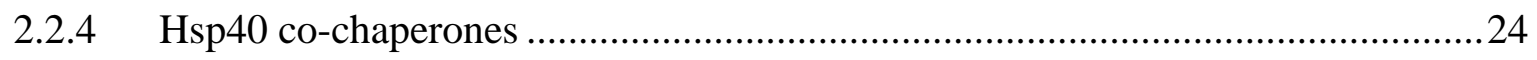

2.2.5 Nucleotide exchange factors (NEFs) ……………………….......................26

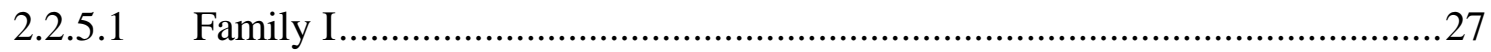

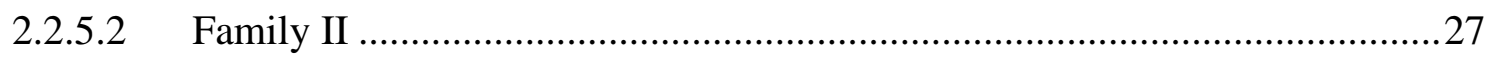

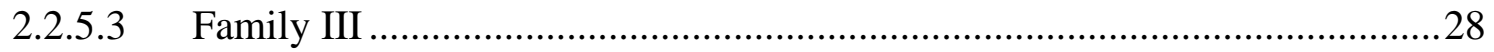

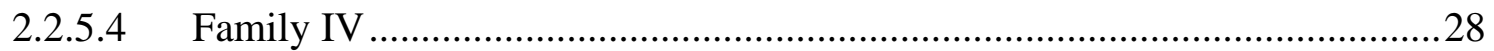

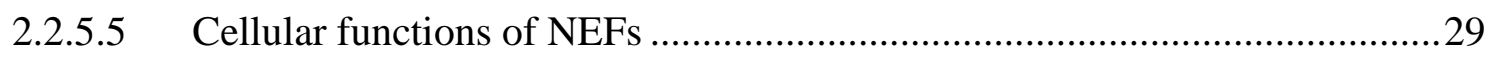

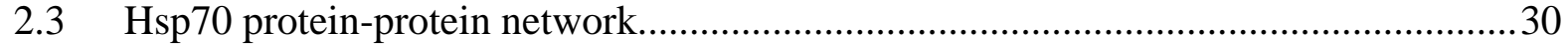

2.3.1 Eukaryotic Hsp70 protein-protein network ..........................................................30

2.3.2 Hsp70 interacting protein (Hip) ...................................................................

2.3.2.1 Predicted domain structure of Hip ............................................................. 32

2.3.2.2 The N-terminal dimerization domain of Hip ................................................... 33

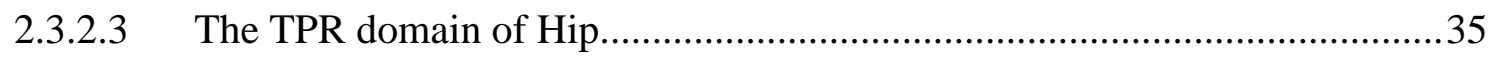

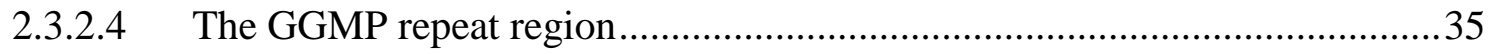

2.3.2.5 The DP domain of Hip..............................................................................

2.3.3 C-terminus of Hsc70 interacting protein (Chip) ................................................36

2.3.4 The Hsp70-Hsp90 organizing protein (Hop) ……….............................................37 
2.3.4.1 Similarities between Hip and Hop …………................................................39

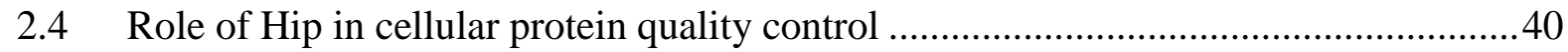

2.4.1 Regulation of progesterone receptor maturation .................................................40

2.4.2 Aggregation prevention in models of neurodegenerative disease...........................41

2.4.3 Hip promotes degradation of misfolded protein ...................................................43

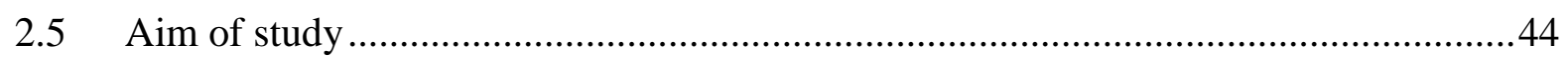

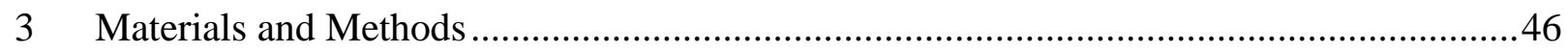

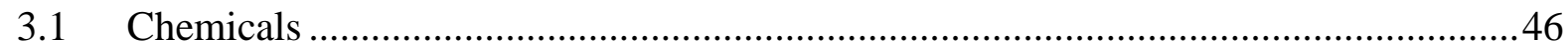

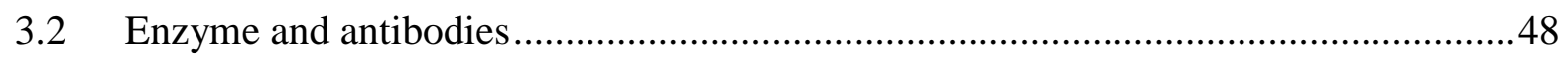

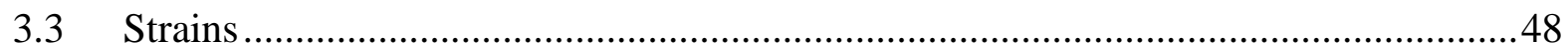

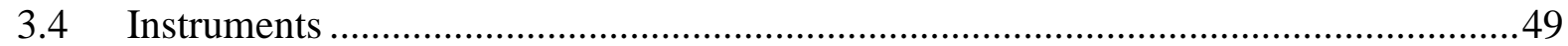

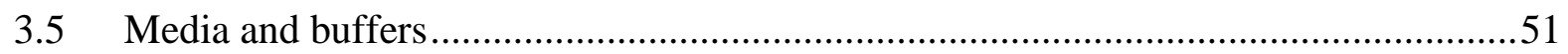

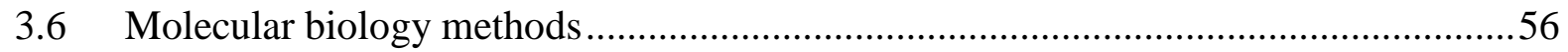

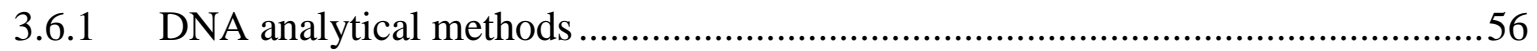

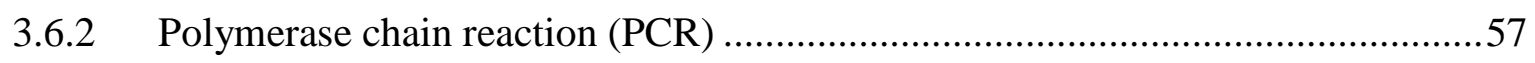

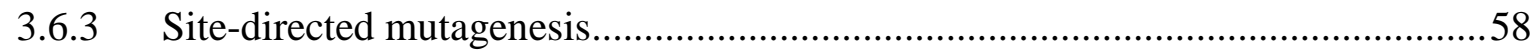

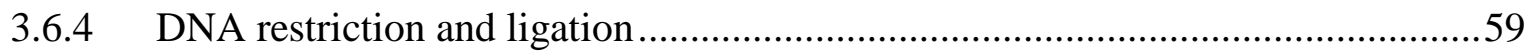

3.6.5 Plasmid DNA and DNA fragment purification..................................................60

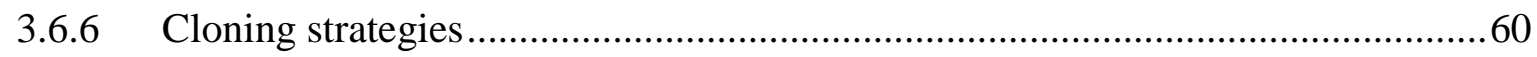

3.6.7 Competent E. coli cell preparation and transformation ......................................61

3.6.7.1 Chemocompetent E. coli cells and chemical transformation...........................61

3.6.7.2 Electrocompetent E. coli cells and electroporation ......................................62

3.6.8 Lithium acetate transformation of S. cerevisiae cells ..........................................63

3.7 Protein biochemical and biophysical methods .........................................................64

3.7.1 Protein expression and purification..................................................................64

3.7.1.1 Hip constructs ......................................................................................6

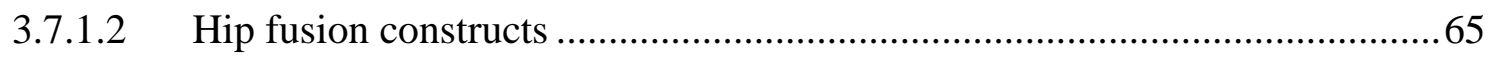

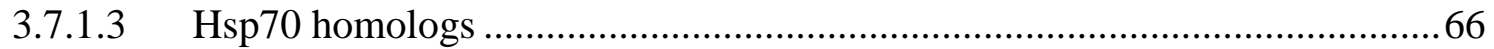

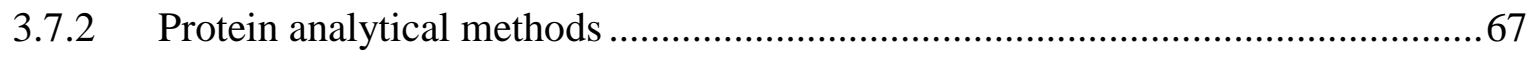

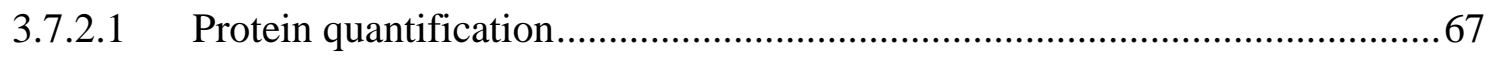

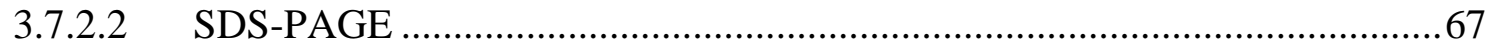




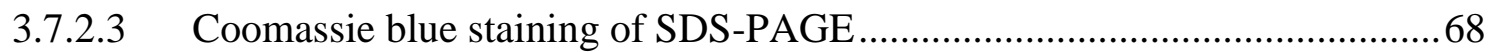

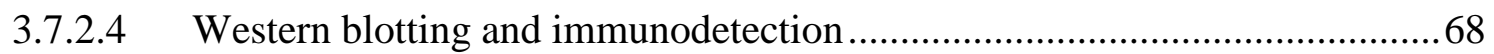

3.7.2.5 Circular dichroism spectroscopy (CD) .....................................................69

3.7.2.6 Small angle X-ray scattering (SAXS) .......................................................69

3.7.2.7 Multi-angle light scattering (MALS) ………............................................ 70

3.7.3 Protein crystallization and structure determination.............................................

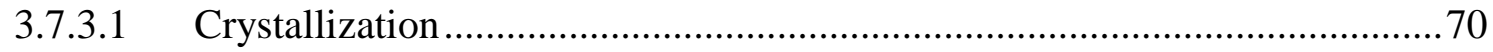

3.7.3.2 Data collection, structure solution and refinement ....................................... 71

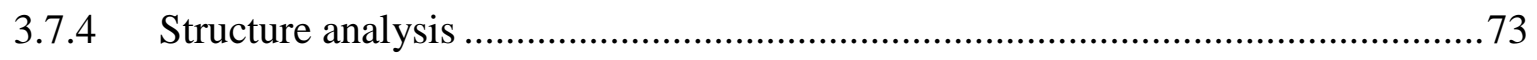

3.7.5 Functional characterization in vitro ...............................................................73

3.7.5.1 Limited proteolysis with Proteinase K.......................................................... 73

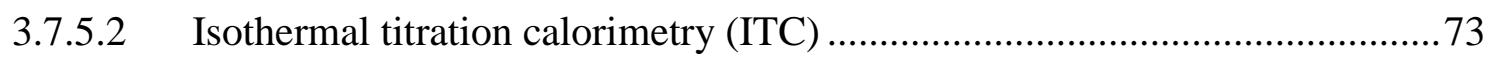

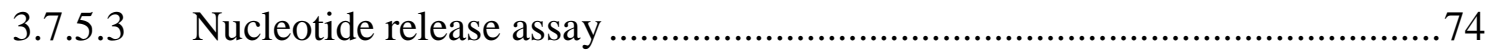

3.7.6 Functional characterization in vivo ..................................................................

3.7.6.1 $\quad \beta$-Galactosidase assay .............................................................................

3.7.6.2 Protein expression analysis by Western blotting .........................................75

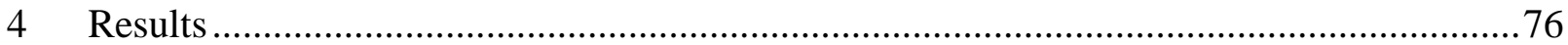

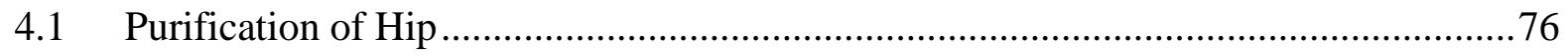

4.2 Characterization of the Hip-Hsp70 interaction by isothermal titration calorimetry ....79

4.2.1 Selectivity of Hip for the ADP-bound state of Hsp70 ………............................79

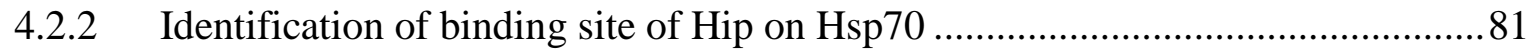

4.2.3 Identification of the Hip minimal Hsp70-binding domain....................................82

4.3 Hip binding decelerates nucleotide release from Hsp70 ........................................... 84

4.4 Nucleotide release is virtually abolished in a Hip-Hsp70 fusion protein......................8 87

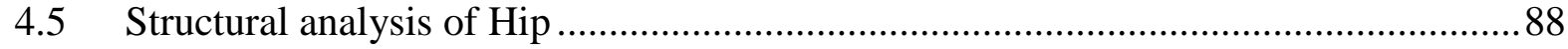

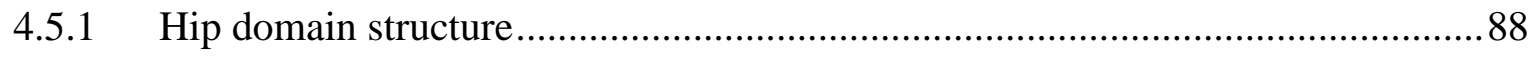

4.5.2 Circular dichroism spectroscopy of Hip domains ................................................89

4.5.3 The DP domain of Hip .................................................................................. 91

4.5.4 Crystallization of the Hsp70-interacting domain of Hip ......................................91

4.5.5 Structure determination and refinement of HipM ..............................................93

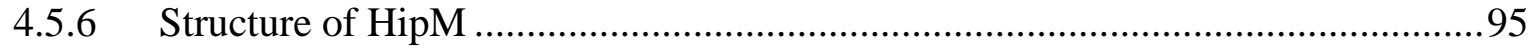


4.5.7 Small angle X-ray scattering (SAXS) .................................................... 97

4.6 Structural analysis of the Hip:Hsp70 complex ...................................................99

4.6.1 Crystallization of the Hip:Hsp70 core complex .............................................99

4.6.2 Structure determination of the Hsp70-Hip fusion protein.................................. 100

4.6.3 Two interaction interfaces between Hip and Hsp70 .................................... 102

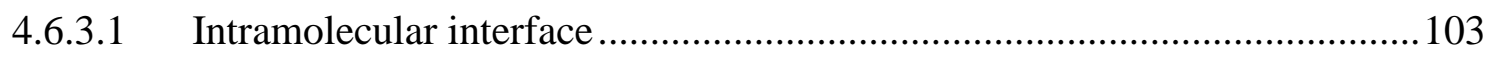

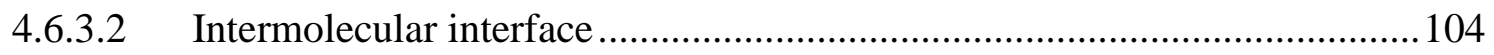

4.6.4 Structure of the Hip:Hsp70 core complex..................................................... 104

4.6.5 Mutational analysis of the binding interface between Hip and Hsp70 .............107

4.7 Interplay between Hip and Hsp70 NEFs ......................................................... 111

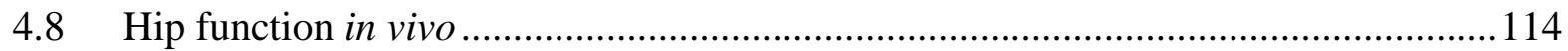

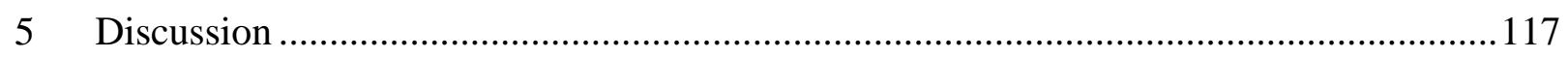

5.1 Comparison of the TPR domains in Hip and Hop............................................... 117

5.2 Tentative model for the overall structure of Hip dimer....................................... 119

5.3 Specific recognition of the Hsp70 ADP state by Hip .......................................... 120

5.4 Mechanism of Hip-mediated stabilization of Hsp70 in the ADP state .....................122

5.5 Antagonism between Hip and NEFs ........................................................ 123

5.6 Possible role of Hip in protein quality control ................................................. 124

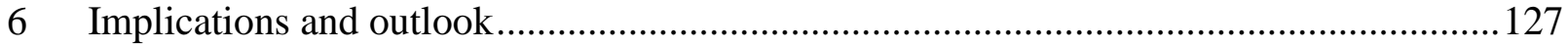

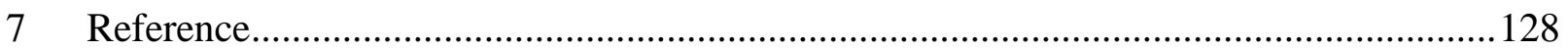

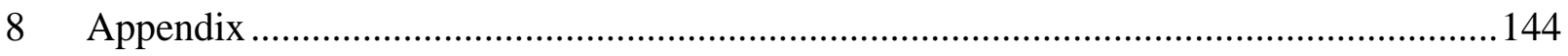

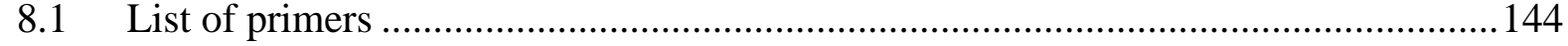

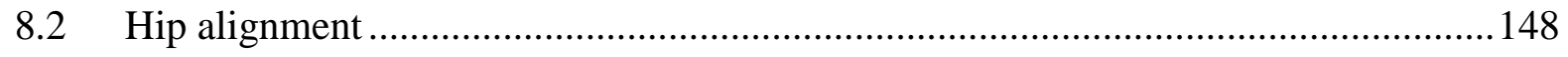

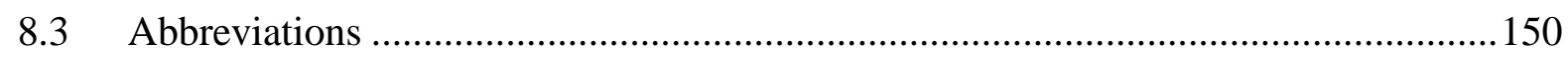

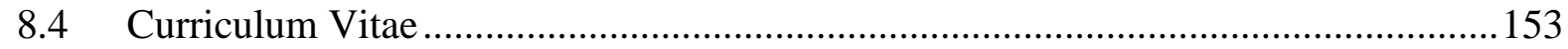




\section{Summary}

Protein must fold into unique three-dimensional structure in order to become functionally active. In the crowded environment of the cell, misfolded proteins easily clump together and form aggregates. Such protein aggregation contributes to neurodegenerative diseases, such as Alzheimer's, Parkinson's and Huntington's disease. Protein aggregation is prevented by specialized proteins called molecular chaperones, which recognize misfolded proteins and promote their productive folding.

The Hsp70 chaperone system is central to the cellular defense against toxic protein aggregation and for maintenance of protein homeostasis. Cytosolic Hsp70 mediates the folding of newlysynthesized and stress-denatured proteins in an ATP-dependent reaction cycle, but also helps in the transfer of misfolded protein species to the degradation machinery. To accomplish protein folding, Hsp70 is regulated by co-chaperones of the J-protein family and by nucleotide exchange factors (NEFs). These stimulate ATP hydrolysis and ADP dissociation, respectively. A further Hsp70 regulator, the protein Hip/ST13 (Hsp70 interacting protein), has been suggested to delay substrate release by slowing ADP dissociation from Hsp70.

Hip was first described in 1995, but its significance for protein homeostasis has only recently been appreciated. Hip, a dimer of $\sim 40 \mathrm{kDa}$ subunits, stabilizes Hsp70-substrate complexes in the ADP-bound state. Hip is conserved in metazoans and functions in stabilizing complexes of Hsp70 with aggregation-prone substrate proteins, including $\alpha$-synuclein in Parkinson's and Tau in Alzheimer's disease.

This study presents an in-depth structural and mechanistic analysis of the Hsp70 regulator Hip. In isothermal titration calorimetry (ITC) experiments Hip bound preferentially to the ADPbound form of Hsp70, however with relatively modest affinity. Binding of Hip slowed the dissociation of a fluorescent ADP analog from Hsp70. Circular dichroism spectrometry and small angle X-ray scattering (SAXS) showed that the structured units in the multi-domain protein Hip are flexibly linked by long tethers, resulting in an extended dimer structure. We determined the crystal structures of the $\mathrm{N}$-terminal dimerization domain and the tetratricopeptide repeat (TPR) middle domain of Hip by experimental phasing at $1.1 \AA$ and $2.6 \AA$ resolution, respectively. For 
the C-terminal DP domain, we constructed a homology model. The TPR middle domain is sufficient for Hsp70 binding. The C-terminal domain presumably mediates contacts to substrate proteins.

Furthermore, we solved the crystal structure of the core complex of Hip with the nucleotide binding domain (NBD) of Hsp70 at $2.7 \AA$ resolution. The complex structure showed that Hip forms a bracket over the ATPase domain of Hsp70, reducing its otherwise dynamic nature and locking the ADP-bound state. As a consequence, dissociation of ADP is retarded and Hsp70client protein complexes are stabilized. Structure-guided mutational analysis targeting the interaction interface fully validated the observed interactions between Hip and Hsp70.

Comparison of the structures of the Hip and NEF complexes suggested that Hip and NEF binding to Hsp70 are mutually exclusive. This was confirmed using a Hip-Hsp70 fusion protein, which forms a functional intramolecular interface. A yeast reporter assay employing the Hsp90 client protein glucocorticoid receptor (GR) showed that Hip must work as a dimer and interact also with the Hsp70-bound substrate protein to compete effectively with NEF binding to Hsp70. Removal of either the dimerization or the DP domain was functionally equivalent to mutations that block interactions with the NBD.

The presented data are in line with a model where association of Hip with specific Hsp70substrate complexes attenuates their active cycling. This mechanism explains why Hip enhances aggregation prevention by Hsp70 and facilitates the transfer of specific proteins to downstream chaperones such as Hsp90 or the proteasome. Pharmacological activation of Hip may prove useful in the removal of potentially toxic protein species. Our findings may thus inform new strategies for the treatment of protein deposition diseases. 


\section{Introduction}

Proteins constitute the largest fraction of the cell interior and fulfill numerous cellular functions, as metabolic enzymes, cellular regulators and in signal transductions. The different types of protein are synthesized as polymers of the common 20 amino acids. The functional properties of a protein are determined by its three-dimensional structure, which is encoded by the amino acid sequence. Therefore, determination of protein structures provides important clues about how proteins perform their function.

\subsection{Protein folding and protein aggregation}

To become biologically functional, newly synthesized proteins must correctly fold to their native state with a unique three-dimensional structure. The information for the structure of a protein is encoded in its primary sequence. This was demonstrated for the first time by Christian Anfinsen, who in 1972 received the Nobel Prize in Chemistry for his pioneering discovery of protein folding in vitro (Anfinsen, 1973; Anfinsen and Haber, 1961; Anfinsen et al., 1954).

Protein folding is not a random process. It follows a direct pathway from unfolded forms to the energetically stable native state. The main driving force of protein folding is the gain of entropy in the overall system through the hydrophobic effect, when hydrophobic regions are buried to form the hydrophobic core and the solvation water is released into the surrounding space. Additional stabilization comes from hydrogen bonding, salt bridges and van der Waals interactions.

The free enthalpy of the native state is around $10-15 \mathrm{kcal} / \mathrm{mol}$ compared to the unfolded state. Along the folding trajectory, metastable non-native intermediates can be formed. The folding properties of a protein can be illustrated by a funnel shaped energy landscape, representing the free-energy potential during protein folding (Fig. 1). 


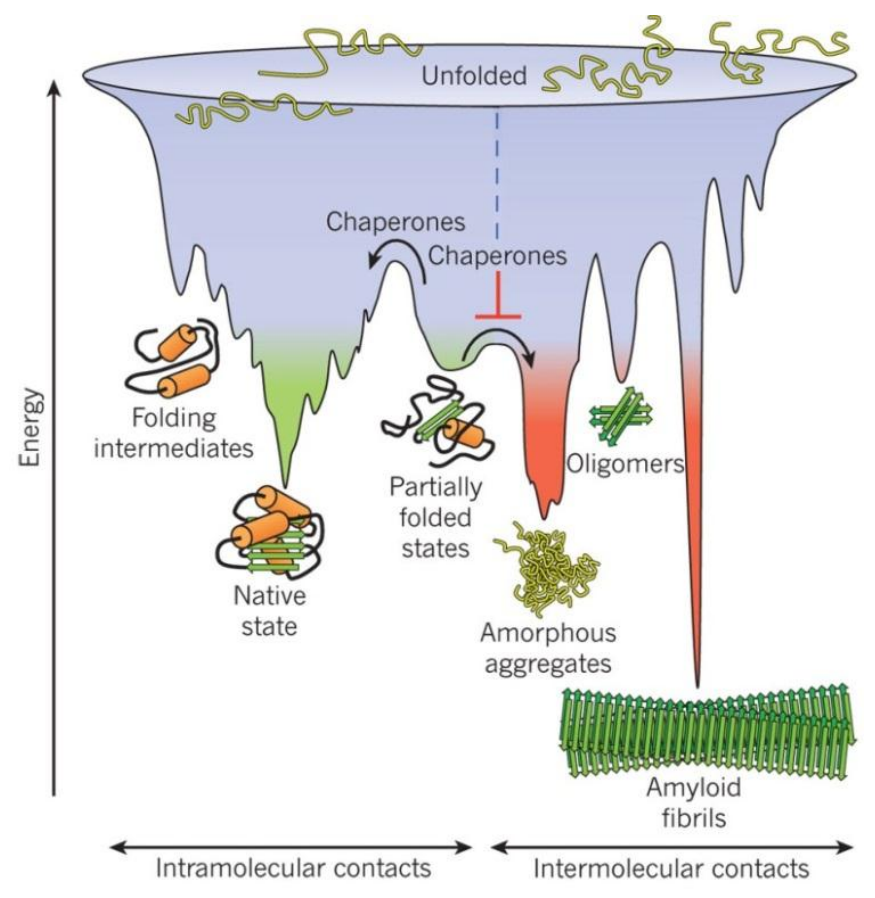

Figure 1: Energy landscape scheme of protein folding and aggregation. Local minima correspond to kinetically trapped intermediate states.

Left panel: Formation of intermediates towards native conformation via intramolecular interactions. Right panel: Misfolding through intermolecular interactions. The red color indicates the intermediates leading towards amorphous aggregates, toxic oligomers or amyloid fibrils via intermolecular contacts. Adapted from the review: (Hartl et al., 2011).

In the crowded environment of the cell, additionally intermolecular interactions contribute substantially to the folding energy landscape (Fig. 1). Partially folded or misfolded proteins tend to aggregate in a concentration-dependent manner, as they transiently expose hydrophobic residues to the solvent. Such aggregates are generally amorphous and their formation prevents productive folding. Alternatively, aggregation can lead to highly ordered, fibrillar aggregates named amyloid fibrils, in which $\beta$-strands run perpendicular to the long fibril axis (right panel in Fig. 1). Such forms appear to be energetically highly stable, perhaps more stable than the native state. These fibrillar amyloid aggregates are highly resistant to the cellular degradation machinery. The occurrence of such aggregates is common to neurodegenerative diseases such as Alzheimer's (AD), Parkinson's (PD), Huntington's (HD) disease as well as Amyotrophic lateral sclerosis (ALS) (Chiti and Dobson, 2006; Olzscha et al., 2011). A large load of misfolded species and aggregated protein are toxic to cells. 
Aggregation of nonnative proteins is a by-product of de novo protein synthesis in vivo, whereby aggregation competes with protein folding. In addition, the aggregation of partially folded proteins and chain compaction is predicted to be enhanced by the crowded macromolecular environment, equivalent to $\mathrm{a} \sim 300-400 \mathrm{mg} / \mathrm{ml}$ protein solution, which increases the affinities between interacting proteins (Ellis, 2001). Crowding also provides a nonspecific force for macromolecular compaction and association (Minton, 2000), including the collapse of protein chains during folding and the interaction of nonnative proteins with molecular chaperones (Martin and Hartl, 1997).

\subsection{Molecular chaperones}

While in the test tube proteins may fold into their native state spontaneously, under cellular condition the folding efficiency is often limited by the side reactions of misfolding and aggregation. To overcome the problem of protein misfolding and aggregation, the cells employ a group of otherwise unrelated proteins called "molecular chaperones".

Chaperones are highly conserved in all three branches of the tree of life, archaea, bacteria and eukaryotes. They only assist the protein folding and assembly process, but are not present in the final structures. Molecular chaperones interact with the nonnative protein during de novo folding and under stress conditions to increase the efficiency of protein folding or re-folding. They are distinguished from folding catalysts such as peptidyl-prolyl isomerases and protein disulphide isomerases (Schmid, 1993; Tuite and Freedman, 1994), while accelerate specific folding steps. Chaperones are constitutively expressed, but under stress conditions, their expression can be greatly increased, hence the term "heat-shock protein" or "stress protein". They are also key players in the regulation of the heat shock response pathway, which is mediated by the transcription factor heat shock factor 1 (Hsf1). Chaperones are mainly divided into five major classes according to molecular weight: small Hsps, Hsp60, Hsp70, Hsp90 and Hsp100.

The molecular chaperone mechanism was originally proposed in 1987 (Ellis, 1987). The universal feature of molecular chaperones is their ability to recognize and bind non-native proteins. Therefore, they are essential for the correct and efficient folding of proteins. They perform mainly three activities: (1) recognizing and interacting with non-native or denatured polypep- 
tide to form a stable chaperone protein complex; (2) preventing aggregation of unfolded and misfolded polypeptides and aiding their refolding; (3) helping the cell to maintain protein homeostasis. For the latter function, chaperones may transfer terminally misfolded proteins to proteolytic degradation by the ubiquitin proteasome system (UPS) or autophagy (Hartl et al., 2011; Rubinsztein, 2006). The cellular folding pathways mediated by molecular chaperones are well established (Fig. 2).

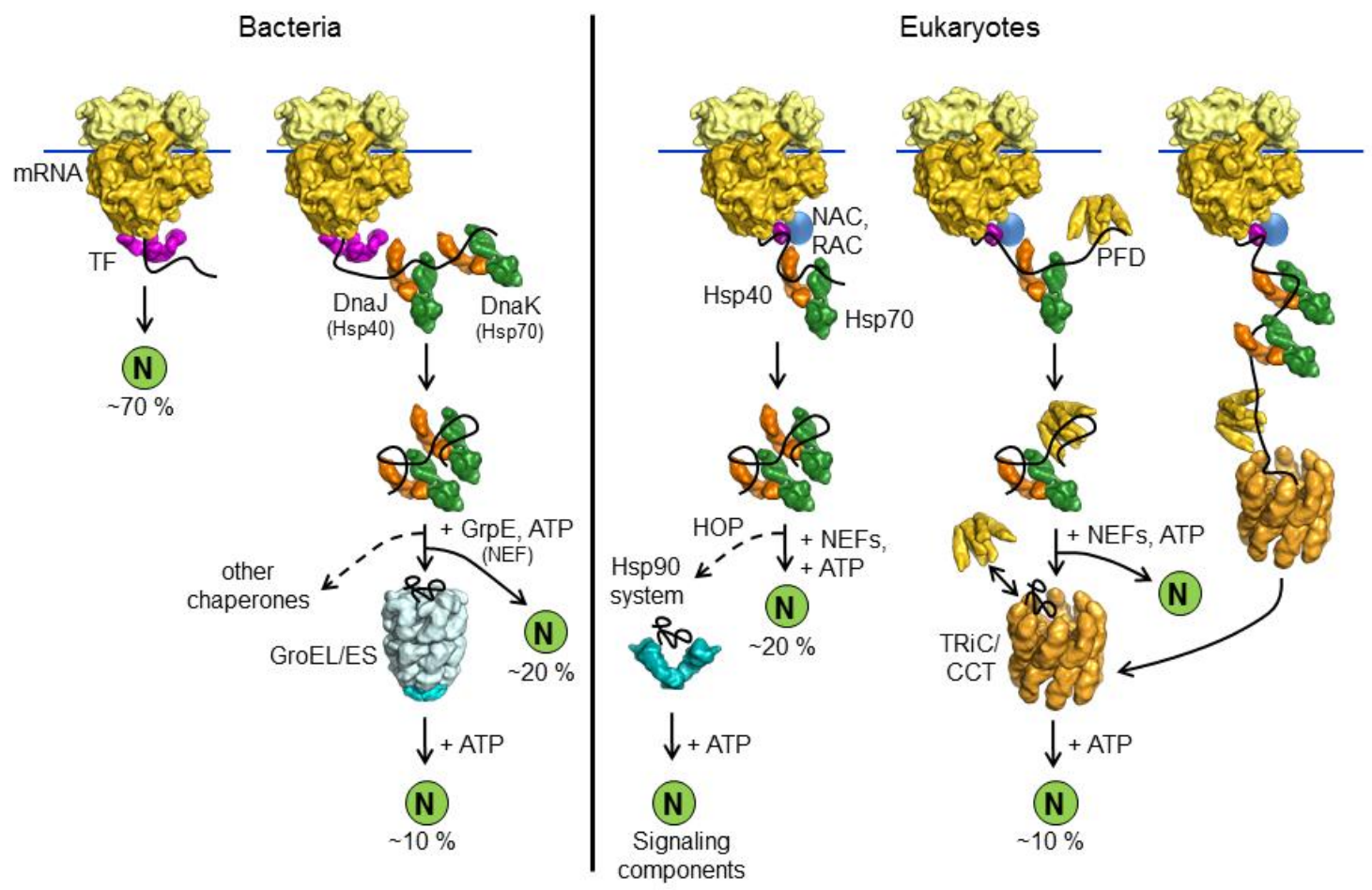

Figure 2: Model of the chaperone-mediated protein folding in the cytosol.

Left panel: In bacteria, newly synthesized polypeptide chains interact with trigger factor (TF) and around 70\% of total proteins may fold directly to native state. Besides that, $20 \%$ of total require the DnaK system, bacterial homo$\log$ of Hsp70. The remaining $10 \%$ are subsequently transferred to the downstream chaperonin GroEL/GroES system to fold to the native state. Right panel: In eukaryotes, nascent chain-associated complex (NAC) interacts with nascent chain, similar to trigger factor. The Hsp70 family functions as second-tier player for longer nascent chains and mediates the co- or post-translational folding. Around $20 \%$ polypeptide can reach their native state through the assistance of ribosome-associated chaperones (RAC), Hsp70 and Hsp40. Hsp70 system also contributes around 10\% protein to downstream chaperones to complete the folding, such as Hsp90 or chaperonin systems. Adapted from the review: (Hartl et al., 2011).

During synthesis of the polypeptide by the ribosome, part of the newly synthesized chain is shielded by the ribosomal exit tunnel. Beyond that, the hydrophobic side chains of partially synthesized polypeptide are exposed to the crowded environment of the cytosol. Thus the ex- 
tended polypeptide needs to be protected from aberrant interactions and misfolding during translation. Therefore, molecular chaperones begin to act cotranslationally. A particular class of chaperones specifically recognizes the newly synthesized nascent-chain emerging from the ribosome. These are ubiquitously present in all domains of life. In the bacterial system, it consists of trigger factor (TF) and the Hsp70 system. In the eukaryotic system, the ribosomeassociated chaperones (RAC) and nascent chain-associated complex (NAC) are involved besides Hsp70. The bacterial trigger factor is the best-characterized ribosome-associated chaperone. After capture by Hsp70, the polypeptide may be handed over to downstream chaperones such as the Hsp60 or Hsp90 systems for further folding and regulation. Therefore, we can summarize that protein folding starts cotranslationally and is completed posttranslationally.

The general understanding of the roles of chaperones in protein folding comes from the study of the chaperonins (Hsp60) and the Hsp70 system, which will be discussed below. The Hsp90 system will also be briefly described as an example for the complex mechanism of molecular chaperones.

\subsubsection{Chaperonins}

Chaperonins are a highly conserved class of macromolecular complexes. They form $\sim 800 \mathrm{kDa}$ large cylindrical folding compartments with a central cavity. Chaperonins are the downstream molecular machinery of Hsp70. The chaperonins have been classified into two families, groups I and II, with GroEL and TRiC as paradigms (Horwich et al., 2007; Tang et al., 2007).

Members of group I are found in bacteria (GroEL) and compartments derived from bacteria in eukaryotes, such as chloroplast (Cpn60) and mitochondria (mt-Hsp60). The chaperonin complexes of group I are composed of 14 subunits, forming two seven-membered rings. The double rings are stacked back-to-back and they cooperate with a co-chaperone (GroES, Cpn10 or mtHsp10), which acts as a lid and covers the top of the chaperonin central cavity (Fig. 3A).

The best-studied and characterized bacterial system is GroEL-GroES, which function as a protein-folding cage (Hartl, 1996; Hartl and Hayer-Hartl, 2002; Horwich et al., 2007). The folding ability of GroEL depends on its molecular architecture (Fig. 3A). GroEL is a double-ring consisting of 14 identical subunits of $\sim 57 \mathrm{kDa}$. Each subunit contains three structural parts: an 
equatorial ATPase domain, an intermediate hinge domain and an apical substrate binding domain. Figure 3B shows the mechanism of GroEL-GroES in detail (Kim et al., 2013).

A)
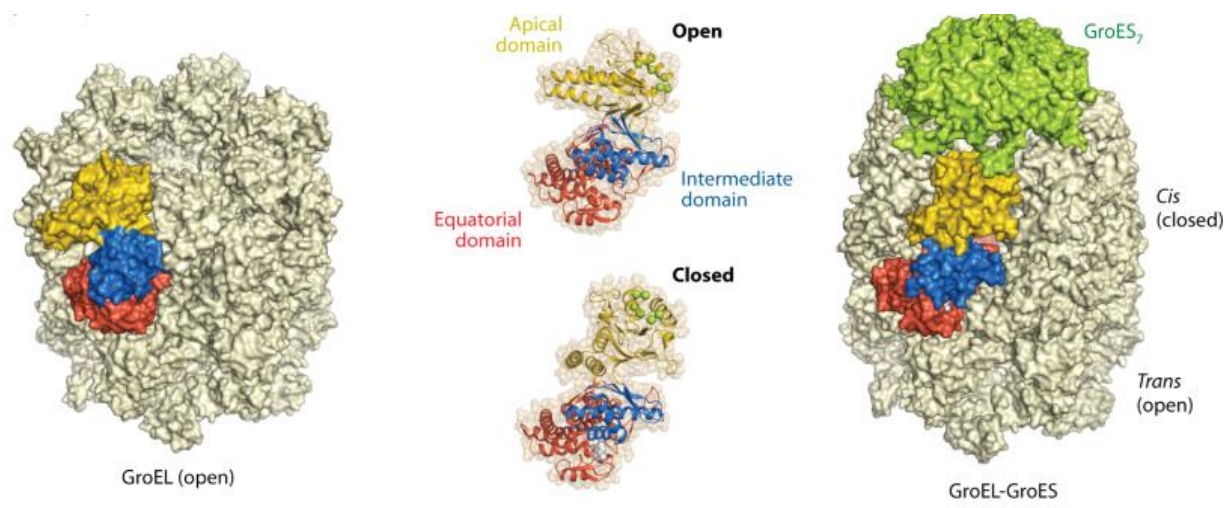

B)

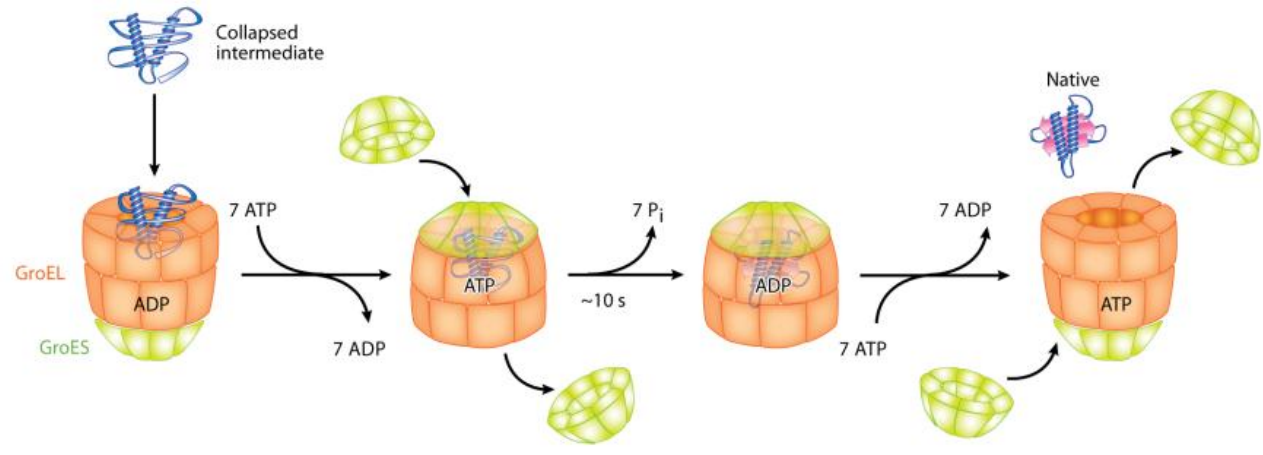

C)
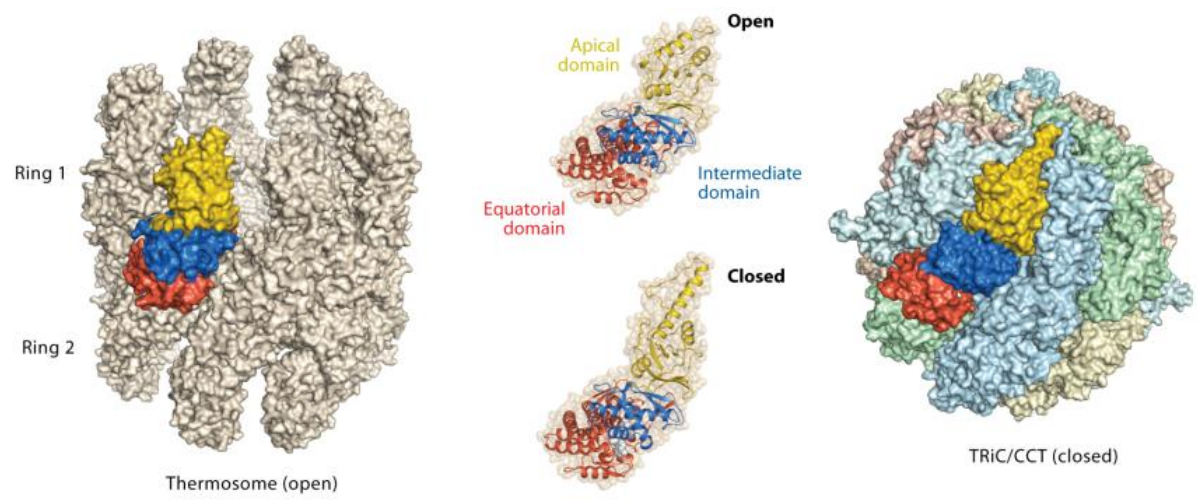

Figure 3: Structures and mechanism of the chaperonin system. 
A) Crystal structure of the group I chaperonin complex, GroEL-GroES. Left panel: The crystal structure of GroEL (PDB accession code 1SS8). Middle panel: Two conformational states of each subunit. The apical, intermediate and equatorial domains are colored in yellow, dark blue and red, respectively. Right panel: The asymmetrical GroEL-GroES complex (PDB accession code 1PF9).

B) GroEL-GroES reaction mechanism. Substrate is bound by the open GroEL cis-ring of the asymmetrical GroEL-GroES complex. Binding of ATP to each of the seven GroEL subunits causes a conformational change in the apical domains. GroES binding traps the substrate in the cage. The encapsulated substrate is free to fold in the GroEL during the time needed to hydrolyze the seven ATP molecules bound to cis-ring ( $10 \mathrm{~s})$. GroES dissociates from the ring and the substrate is released.

C) Crystal structures of group II chaperonins. Left panel: Open form of the homo-oligomeric thermosome (PDB accession code 3KFK). Middle panel: Subunit CCT3 of TRiC in two states, which is colored as follows to indicate its domain structures (equatorial nucleotide-binding domain in red; intermediated hinge domain in green and the apical substrate-binding domain in yellow). Right panel: Closed form of TRiC/CCT (PDB accession code 4D8Q).

The substrate is delivered from the upstream DnaK (Hsp70) system and is trapped on the GroEL ring. It may assume an extended or compact conformation. ATP-dependent apical domain movements result in sequential stretching and re-modeling the partially folded region. The binding of GroES traps the substrate in the cage. GroES is a dome-shaped homoheptamer of $\sim 10 \mathrm{kDa}$ subunits and regulates the ATPase activity of GroEL (Horwich et al., 2007; Xu et al., 1997). The substrate folds in the chaperonin cavity. After ATP hydrolysis, the substrate is released either in native state or as an incompletely folded intermediate state. The incompletely folded substrate is re-bound to GroEL and will start a new cycle. The whole folding process is regulated by ATP and GroES. Binding of GroES leads to allosteric conformational change of GroEL and results in an expansion of the central cavity (Hartl and Hayer-Hartl, 2009). Unlike Hsp70, GroEL recognizes a molten globule, locally expanded form of substrate, and promotes folding through cycles of encapsulation. Due to the volume of the cavity, the size of the trapped substrate is limited, ranging from $20-50 \mathrm{kDa}$. They have been identified by a proteomics analysis of GroEL/ES binding partners (Kerner et al., 2005).

Group II chaperonins such as thermosome and TRiC (TCP-1 ring Complex) exist in archaea and the eukaryotic cytosol (Fig. 3C). TRiC is also called CCT (Chaperonin Containing TCP-1). In contrast to group I chaperonins and thermosomes, TRiC is not stress inducible (Horwich et al., 2007). Though all chaperonins share a common cylindrical structure, substantial differences exist between groups I and II. TRiC contains eight different subunits per ring and each subunit is $\sim 60 \mathrm{kDa}$. In addition, group II TRiC subunits contain $\alpha$-helical protrusions that extend from the apical domain of the ring, which has been described as a built-in lid. Therefore, TRiC work independent from an additional GroES-like co-chaperone. Notably, TRiC subunits share se- 
quence homology with GroEL at the ATP binding site, but not the substrate binding site. Therefore, TRiC was assumed to be specialized for a small subset of substrate proteins (Gomez-Puertas et al., 2004). A recent study suggested however that $10 \%$ newly synthesized proteins are TRiC substrates, ranging from the obligate substrate such as actin and tubulin to tumor suppressors (Yam et al., 2008). The broad range of substrate suggested that TRiC may work by a mechanism different from group I GroEL-GroES system.

\subsubsection{The Hsp90 system}

Similar to Hsp70, Hsp90 is a highly conserved and essential molecular chaperone in the mammalian cytosol and nucleus. Hsp90 functions in the downstream of Hsp70 and associates with non-native protein substrates. It mediates the conformational regulation of a wide range of client proteins involved in cell cycle control, apoptosis and especially signal transduction (Burrows et al., 2004; Pearl and Prodromou, 2000). The glucocorticoid receptor (GR) was identified as the first client protein of Hsp90 (Sanchez et al., 1985). Signaling components such as steroid-hormone receptors including progesterone receptor (PR), androgen receptor (AR) and kinases are the best-understood clients of Hsp90 (McClellan et al., 2007; Picard, 2006). Hsp90 is also essential for the cell's response to stress and is a key player in maintaining cellular homeostasis (Taipale et al., 2010).

Though a comprehensive understanding of the cellular functions and underlying mechanism of Hsp90 is still elusive, the crystal structures of full-length Hsp90 in complex with its cochaperones provided deep insights into the structural dynamics and the regulation of Hsp90 (Ali et al., 2006; Pearl and Prodromou, 2000; Shiau et al., 2006). 


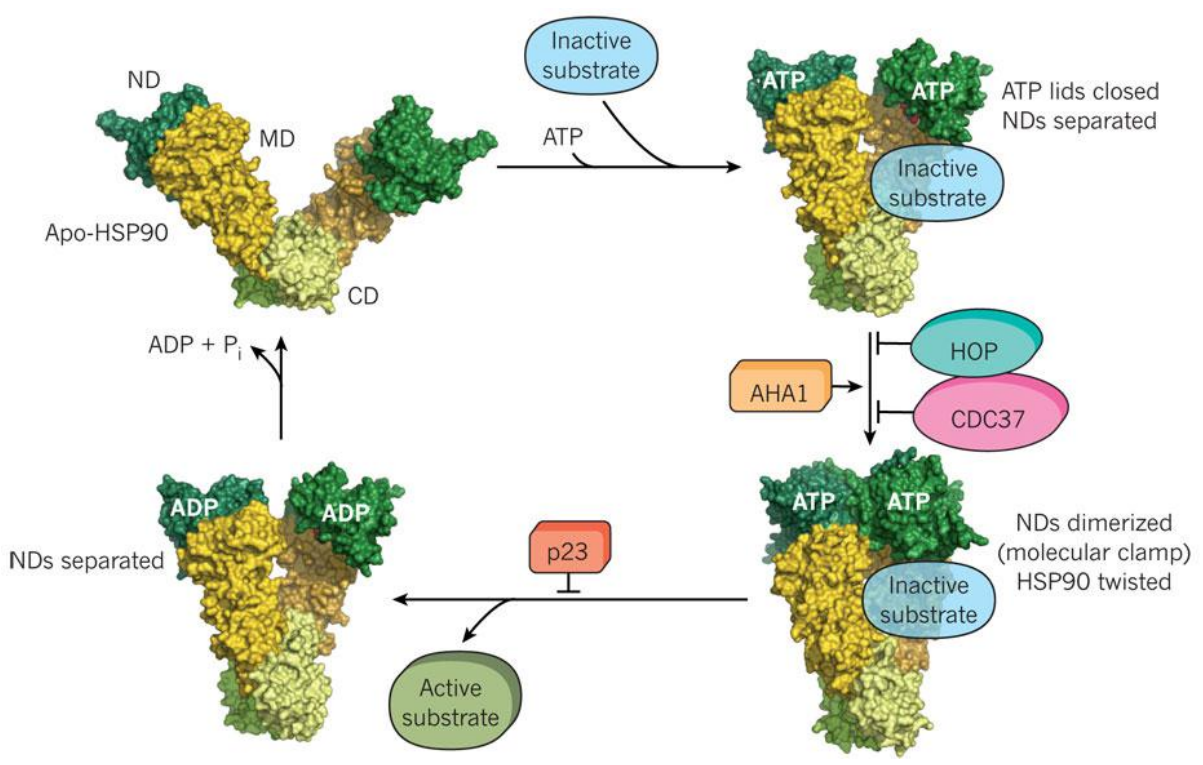

Figure 4: ATPase cycle of Hsp90.

Upon ATP binding, the N-terminal ATPase domain (ND) undergoes a conformational rearrangement, leading to the dimerization of ND. The dimerization forms a "molecular clamp" and results in a compact Hsp90 dimer. The ATP-bound state is metastable and committed to ATP hydrolysis. Transition to the ATP-hydrolysis competent state is regulated by co-chaperones. After ATP hydrolysis, the NDs dissociate and the protomers in the Hsp90 dimer remain connected by the $\mathrm{C}$-terminal domain (CD). It has been suggested that the inactive substrate interacts mostly with the middle domain (MD) and is conformationally activated, when Hsp90 proceeds through the ATPase cycle. Adapted from the review: (Hartl et al., 2011).

Hsp90 exists as a flexible dimer, connected by its C-terminal domains. Each monomer consists of three domains: an N-terminal nucleotide binding domain (ND), followed with a middle domain (MD) and C-terminal dimerization domain (CD) (Figure 4). The ND possesses a deep nucleotide binding pocket, where ATP is bound in an unusual kinked manner. Similar to other chaperones, Hsp90 mediates ATP hydrolysis-dependent folding of substrates. The ATPase activity is essential for the function of Hsp90. Figure 4 shows the ATPase cycle of Hsp90 with its highly dynamic conformational changes. Besides the C-terminal dimerization domain, ATP binding promotes the dimerization of $\mathrm{ND}$, resulting in a compact dimeric conformation of Hsp90. In this compact dimer, two monomers twist around each other and the NDs and MDs are also tightly associated. ATP hydrolysis leads to the dissociation of the NDs, resulting in the open conformational state of Hsp90, which enables the recognition of various substrates. Because of the broad extent of substrate specificities and the different structures of the known 
substrates, Hsp90 is assumed to have multiple binding sites to substrates and the identification of binding sites on Hsp90 is still ongoing (Wandinger et al., 2008).

In addition, the CD domain contains an EEVD peptide motif at the end of C-terminus, which serves as a specific binding site for several co-chaperones, such as Hop and Chip, which bind to Hsp90 via TPR domains. Hop facilitates the cooperation between Hsp90 and Hsp70. Chip facilitates the cooperation of Hsp90 and proteasome. Hop inhibits $\mathrm{N}$-terminal dimerization of Hsp90. It works as an adaptor for Hsp70 and Hsp90 and participates in steroid hormone receptor maturation by targeting clients to Hsp90 and forming the Hsp90-client complex. Moreover, Hsp90 is also targeted by several other co-chaperones without TPR domains, such as Cdc37, Aha1 and p23, which interact with Hsp90 in a sequential manner to regulate the chaperone machinery (Pratt and Toft, 2003; Smith, 1993). Cdc37 delivers specific kinase substrates to Hsp90 and acts as an ATPase inhibitor. Thereby, Cdc37 is thought to keep Hsp90 in an open state for transfer of the kinase. Conversely Aha1 stimulates ATP hydrolysis. In contrast to Aha1, p23 stabilizes the compact dimeric form of Hsp90 before ATP hydrolysis. In summary, the cochaperones may control the conformational changes of Hsp90 and thereby modulate specific substrate processing by Hsp90.

It is widely recognized that the Hsp90 system is multifunctional and multicomponent chaperone machinery in the protein folding apparatus of the cell. In addition to client protein folding, Hsp90 has been shown to cooperate with the degradation machinery of the cell. This lead to a particular focus on Hsp90 with regard to pharmacological modulation (Schneider et al., 1996). Since it is responsible for the maturation of approximately 200 client proteins, among which several are oncoproteins (Workman et al., 2007), Hsp90 has gained attention as a critical target in cancer therapy, especially through the use of small compounds like geldanamycin that act as specific inhibitors of Hsp90. These drugs bind to the ATP binding pocket of Hsp90 blocking ATP binding. Thereby they specifically inhibit the ATPase function and arrest the Hsp90 functional cycle. The growing number of Hsp90 inhibitors has been evaluated in preclinical and clinical trials, by which a proof-of-concept has been established (Kim et al., 2009; Sharp and Workman, 2006; Workman et al., 2007). 


\subsubsection{The Hsp70 system}

\subsubsection{Diversity of Hsp70 isoforms}

Hsp70s are the most ubiquitous chaperones and occur in all three domains of life: archaea, bacteria and eukaryotes (Hartl and Hayer-Hartl, 2002). The archaeal and bacterial Hsp70 is named DnaK. The eubacterium Escherichia coli has three Hsp70 isoforms (DnaK, HscA and HscC). The unicellular eukaryote S. cerevisiae contains four non-ribosome associated Hsp70s (Ssa1-4) and three ribosome-associated Hsp70s (Ssb1, Ssb2, Ssz1) in the cytosol, and organelle-specific isoforms in the endoplasmic reticulum (ER) and mitochondria (Lindquist and Craig, 1988; Nelson et al., 1992). In mammals, the constitutively expressed and stress-inducible isoforms are known as heat shock cognate 70 (Hsc70) and Hsp70, respectively, but more isoforms exist, 13 in total (Systematic names start with HSPA, followed by a number.). Hsc70 and Hsp70 share about $80 \%$ sequence identity. The Hsp70s present in mitochondria and ER are known as mtHsp70/Grp75 and Bip/Grp78/Kar2, respectively. mtHsp70 is involved in the import of nuclear-encoded proteins into mitochondria. In the ER lumen, Bip is responsible for protein folding and quality control of proteins of the secretory pathway. Additional versatility is achieved by the employment of a multitude of Hsp40 family proteins ( $\mathrm{J}$ proteins) and nucleotide exchange factors (NEFs) present in the eukaryotic cell, which act as co-chaperones. They control ATP hydrolysis of Hsp70 and recruit Hsp70 to specific substrate proteins.

\subsubsection{Cellular functions of Hsp70}

The Hsp70s have essential roles in protein metabolism under normal and stress conditions. They are central to many cellular processes, such as de novo protein folding, membrane translocation and misfolded protein degradation (Hartl and Hayer-Hartl, 2002; Neupert and Brunner, 2002; Ryan and Pfanner, 2001; Wang et al., 2013). One of the important roles of Hsp70 is to assist protein folding and turnover. Many neurodegenerative diseases involve aberrant accumulation of misfolded protein, and previous studies have linked Hsp70 to this process. In vitro studies showed that Hsp70 together with its co-chaperone Hsp40 can partially suppress the aggregation of huntingtin (htt) in the early prefibrillar state (Muchowski et al., 2000). Consistently, Hsp70 was found to co-localize with polyQ aggregates implicated in polyQ expansion diseases, and overexpression of Hsp70 and Hsp40 reduces polyQ aggregation and cytotoxicity 
(Jana et al., 2000). Moreover, overexpression of Ssa1 (yeast homolog of Hsp70) decreases aggregation of htt and increased its SDS solubility (Krobitsch and Lindquist, 2000). Furthermore, Hsp70 has been reported to be sufficient to inhibit amyloid fibril formation by $\alpha$-Synuclein, even in the absence of co-factors (Dedmon et al., 2005). Intriguingly, Hsp70 is able to recruit the co-chaperone ubiquitin ligase Chip for the ubiquitination of Tau, in contrast to Hsc70. One recent genetic study reported that Hsp70 directly stabilizes lysosomes and plays a key role in Niemann-Pick disease (Kirkegaard et al., 2010). All of the versatility of Hsp70 builds from its basic function. It binds the hydrophobic regions of unfolded polypeptides in an ATP-dependent manner.

In addition, Hsp70 appears to be a key component of cellular protein quality control. Hsp70 is abundantly expressed in many human tumors and this often correlates with metastasis and poor outcome in cancer patients (Brodsky and Chiosis, 2006; Patury et al., 2009). Up-regulated Hsp70 function is critical for the growth and survival of different human tumor cell lines (Brodsky and Chiosis, 2006; Patury et al., 2009; Powers et al., 2008). In light of the ample structural data available on this molecular chaperone, Hsp70 appears as an attractive putative drug target for the treatment of cancer.

\subsubsection{Structure and working cycle of Hsp70}

All the Hsp70 forms are comprised of two domains, a $45 \mathrm{kDa}$-terminal nucleotide binding domain (NBD) and a $\sim 25 \mathrm{kDa}$ C-terminal substrate binding domain (SBD), which contains a $\sim 10 \mathrm{kDa} \alpha$-helical "lid" domain and a $\sim 15 \mathrm{kDa} \beta$-sandwich (Fig. 5A). The NBD and SBD are connected via a highly conserved hydrophobic linker with 10-12 residues (Vogel et al., 2006). Structural studies have revealed that the NBD of Hsp70 is composed of two lobes, I and II. Each lobe consists of two subdomains named IA, IB and IIA, IIB, respectively. The two lobes form a heart-shaped structure with a large cleft at the bottom, where the nucleotide-binding site is located. The two subdomains look like a clamp that locks the nucleotide within the cleft. The nucleotide binding is coordinated by residues derived from all four subdomains. Subdomain IIB plays an essential role in the dissociation of nucleotide. It was well-known that NBD and SBD of Hsp70 in ADP-bound state are structurally and functionally almost independent (Chang et al., 2008; Swain et al., 2007). Conversely, in the ATP state, many biochemical and NMR 
studies have suggested that NBD and SBD contact with each other (Buchberger et al., 1995; Mayer, 2010; Swain et al., 2007). However, the structure of ATP-bound full length Hsp70 had remained elusive until the crystal structure of an intact Hsp70 became recently (Kityk et al., 2012; Qi et al., 2013). In the ATP-bound state, the NBD and SBD form extensive contacts, which cause a dramatic conformational change in the SBD. The $\alpha$-helical lid is separated from the $\beta$-sandwich domain and packs against the NBD, leading to an accessible peptide binding site. In the structure of an isolated SBD bound to a hydrophobic peptide, the $\beta$-sandwich harbors the peptide binding site, and the tightly associated $\alpha$-helical domain forms a lid over the bound peptide (Zhu et al., 1996).

As a molecular machine, Hsp70 performs its function through crosstalk between ATPase activity in the NBD and substrate binding in the SBD. Hsp70 binds the hydrophobic polypeptide with its SBD, hydrolyzes ATP and then directs its substrate into a variety of distinct fates (Evans et al., 2010). Substrates of Hsp70 are thought to include newly synthesized linear polypeptides and partially folded proteins.

A)

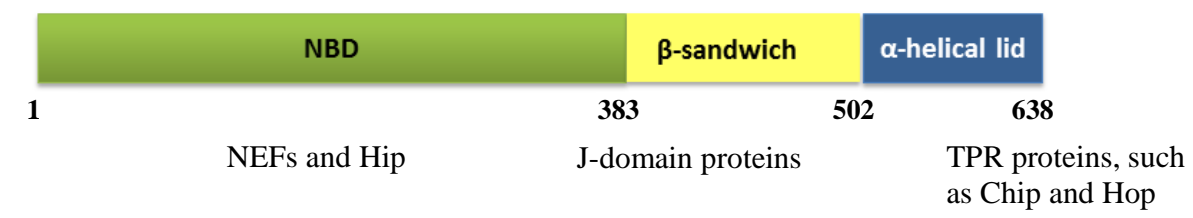

B)
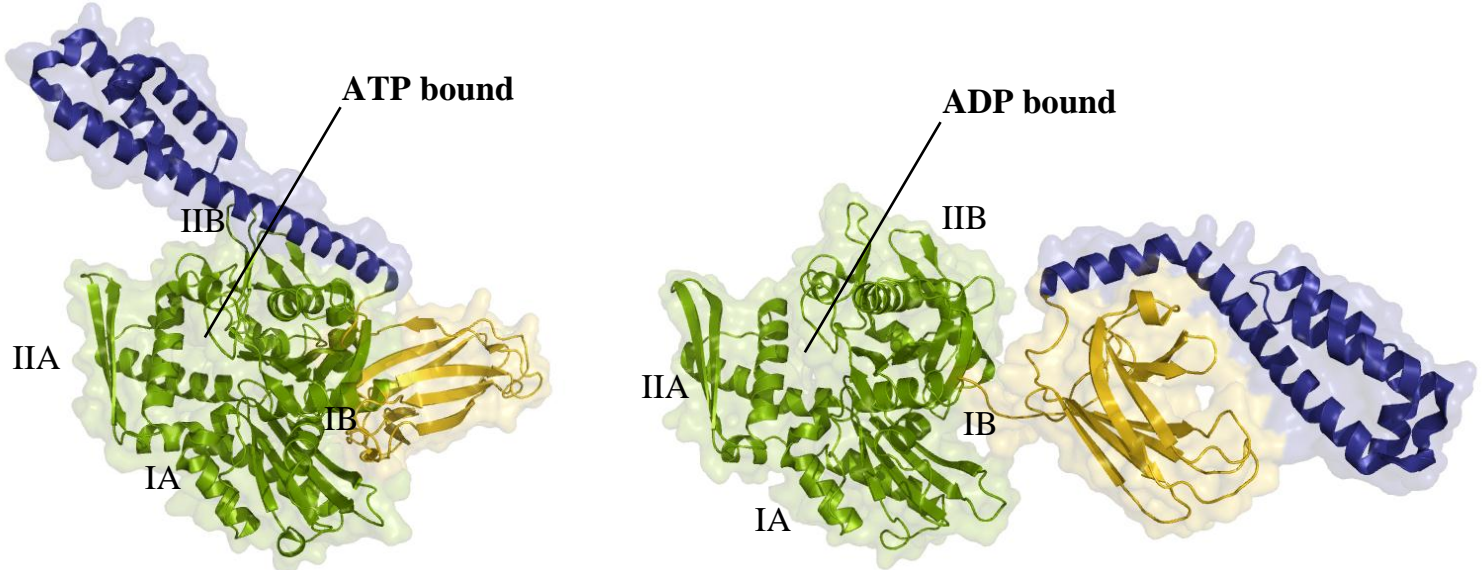
C)

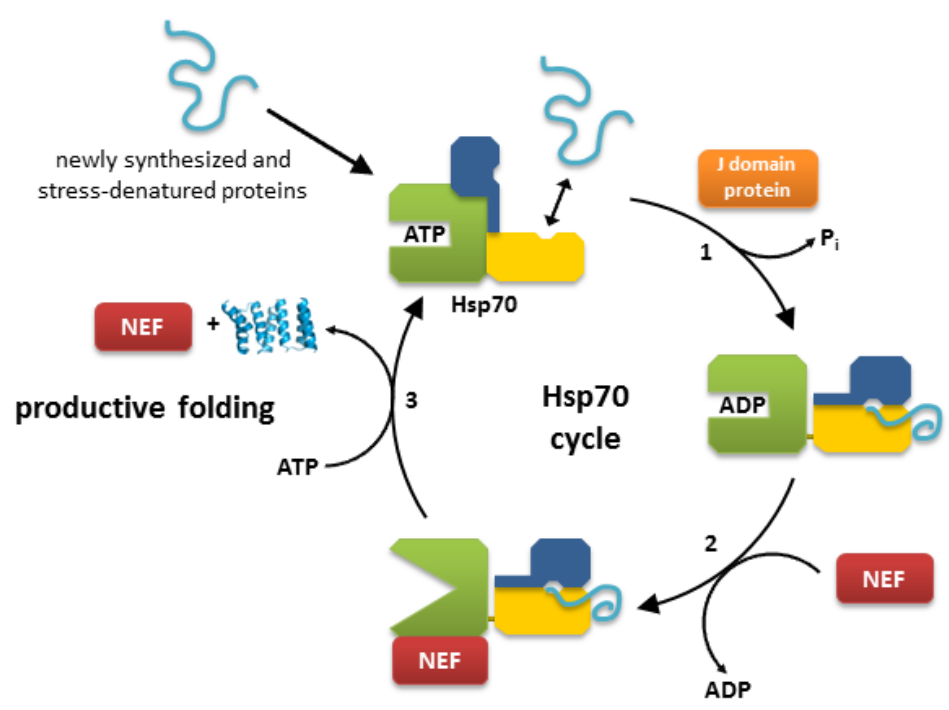

Figure 5: Structure and working cycle of Hsp70.

A) Schematic representation of domain architecture of Hsp70 in DnaK E.coli. Hsp70 consists of two domains, the nucleotide-binding domain (NBD) in green color and the substrate-binding domain (SBD), which is composed of a $\sim 15 \mathrm{kDa} \beta$-sandwich domain and $10 \mathrm{kDa} \alpha$-helical "lid" domain, shown in gold and dark blue, respectively. Co-chaperone interaction sites with Hsp70. Only eukaryotic Hsp70 in cytosol contain an EEVD sequence at their C-terminus, which mediates the interaction with tetratricopeptide repeat TPR proteins, such as C-terminus of Hsc70 interacting protein (Chip) and Hsp70-Hsp90 organizing protein (Hop).

B) Left panel: Crystal structure of bacterial Hsp70, DnaK in the ATP-bound open state. Right panel: Closed state in ADP-bound of E. coli DnaK (PDB: 2KHO), which was solved by NMR spectroscopy using the crystal structures of the individual domains.

C) The ATP-dependent reaction cycle of Hsp70, regulated by J-domain proteins and nucleotide-exchange factors (NEFs).

Hsp70 is an allosteric protein. It has two conformational states with distinct substrate binding properties: the open state with low affinity to substrate, and closed states with high affinity (Fig. 5B). Substrate binding stimulates ATP hydrolysis, while ATP binding enhances substrate release. Figure 5C shows the model of Hsp70 working cycle, which is well-understood for the bacterial Hsp70 homolog DnaK. To accomplish the ATPase cycle, distinct classes of cochaperones are required. The J-domain protein DnaJ is required for stimulation of ATP hydrolysis and the nucleotide exchange factor (NEF) GrpE for ADP dissociation from Hsp70. The collaboration with these important co-chaperones drives the transition of Hsp70 between the ATP and ADP states. ATP binding to the Hsp70 NBD increases the rate of substrate binding to SBD. Conversely, interactions between SBD and substrate increase the rate of ATP hydrolysis 
through communication between the two functional domains (Mayer et al., 2000). In the absence of J-domain proteins, Hsp70 is predominantly in the ATP-bound state, since the intrinsic ATPase activity of Hsp70 is low (Schmid et al., 1994).

The elucidation of the DnaK mechanism in detail has provided the paradigm for the function of the eukaryotic Hsp70 chaperone system. There are additional co-chaperones to control substrate binding by independently binding to specific substrates, leading to a kind of substrate selection for Hsp70 (Kota et al., 2009; Vos et al., 2008). Recently, a novel protein Hikeshi was discovered that recognizes the ATP-bound state of Hsp70 and mediates its transport into the nucleus under heat-shock stress condition (Kose et al., 2012). Hsp70 distribution between cytosol and nucleus might be important for the decision between re-folding and degradation.

\subsubsection{Hsp40 co-chaperones}

The working cycle of Hsp70 starts with the action of J-domain proteins. They are also referred to as Hsp40, which induce tight binding of substrate to Hsp70. Hsp40 proteins have been well established to recognize and stabilize unfolded substrates for delivery to the hydrophobic pocket of Hsp70 (Gamer et al., 1996). They were proposed to interact with the NBD and the highly conserved hydrophobic linker of Hsp70 through its J-domain and to coupling substrate with its C-terminal substrate binding domain (Karzai and McMacken, 1996). Furthermore, substrate and J-domain protein binding synergistically stimulated ATP hydrolysis by Hsp70 (Laufen et al., 1999).

J-proteins are a heterogeneous group of multi-domain proteins. Though they are thought to act as co-chaperones of Hsp70, their number of homologs far exceeds that of Hsp70. There are six J-domain proteins in E. coli, 20 in S. cerevisiae, 33 in C. elegans and presumably 44 in human cells (Qiu et al., 2006). Based on their domain composition, they are subdivided into three classes as depicted in Figure 6: Type I: DnaJA (DnaJ E.coli, Ydj1 yeast, HDJ-2 human), Type II: DnaJB (Sis1 yeast, HDJ-1 human) and Type III: DnaJC (pyJ polio virus) (Cheetham and Caplan, 1998). Type I has full domain conservation with E. coli DnaJ, which contains four domains: N-terminal J-domain, followed by a glycine-phenylalanine rich region, a Zn-binding domain and a C-terminal domain. The $\alpha$-helical J-domain is composed of $\sim 70$ amino acids and 
responsible for the interaction with Hsp70 (Wall et al., 1994). Type II is highly similar to Type I apart from missing the Zn-binding domain. Type III is more divergent as it only shares similarity to the J-domain segment of types I and II. Since the C-terminal domains are highly divergent, this class is less explored so far.

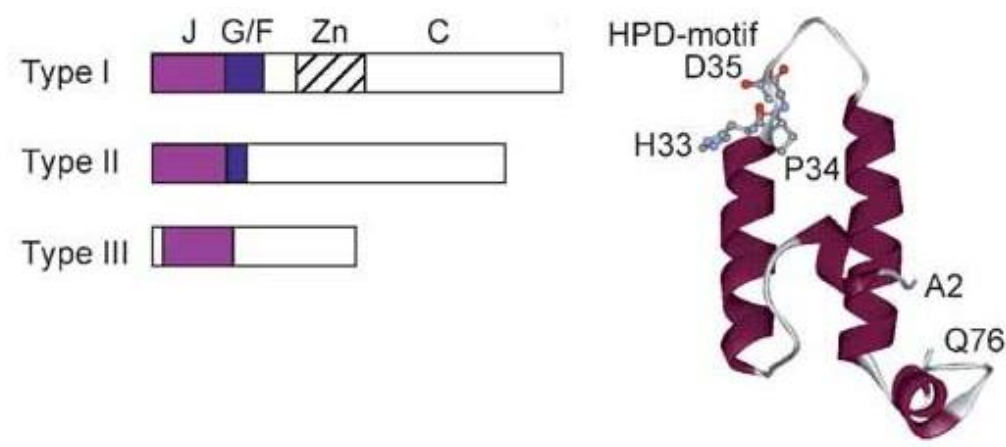

Figure 6: J-domain protein family.

Left panel: Schematic representation of domain architecture of three subfamilies of J-proteins. J: N-terminal Jdomain (magenta color). G/F: Gly-Phe rich region. $\mathrm{Zn}$ : $\mathrm{Zn}^{2+}$ binding domain. C: C-terminal domain of homology. Right panel: Ribbon representation of the NMR structure of J-domain of DnaJ in E. coli. Adapted from review: (Mayer and Bukau, 2005)

The structure of the $\mathrm{J}$-domain composed of four $\alpha$-helices is common to all $\mathrm{J}$-domain proteins (Fig. 6). The central two long anti-parallel $\alpha$-helices are connected by an exposed loop with a conserved signature sequence, histidine-proline-aspartate (HPD). NMR and mutagenesis studies revealed that both of these two anti-parallel $\alpha$-helices are necessary for the binding to Hsp70 and modulating ATP hydrolysis (Landry, 2003). The interactions between J-domain and Hsp70 trigger an allosteric conformation change, which accelerate ATP turnover by approximately seven-fold (Pierpaoli et al., 1997). In addition, a crystallographic study suggested that J-domain stimulated ATPase activity by directing the Hsp70 linker between NBD and SBD to displace their interactions (Jiang et al., 2007). Furthermore, binding of J-domain to Hsp70 displaced the SBD from NBD, which may activate Hsp70 for substrate binding through the SBD flexibility to capture diverse substrates (Jiang et al., 2007). Despite of its function in substrate targeting to Hsp70, the J-domains has been reported that it can stimulate the binding of Hsp70 to polypeptides (Misselwitz et al., 1998). The solid binding assay demonstrated that J-domain binding to Bip and activated Bip to bind more substrates, suggesting J-domain activation broadened the substrate binding range of Bip (Misselwitz et al., 1998). Therefore, it could be proposed that 
substrate specificity of Hsp70 could be largely influenced by its partner, J-proteins. Moreover, the cooperation of J-domain proteins with Hsp70 can prevent the aggregation of unfolded protein.

\subsubsection{Nucleotide exchange factors (NEFs)}

A distinct class of co-chaperones involved in the Hsp70 ATPase cycle is the nucleotide exchange factors (NEFs), which catalyze ADP dissociation. Nucleotide dissociation is a crucial step and therefore it is regulated by a variety of NEFs in eukaryotes. Four major NEF families in the cellular cytosol are classified as: GrpE-like, BAG proteins, HspBP1 and Hsp110 (Harrison, 2003; Kabani et al., 2002; Kabbage and Dickman, 2008; Shaner and Morano, 2007). In the bacterial system, ADP is firmly trapped in DnaK. In the absence of GrpE, the bound nucleotide cannot be released effectively. This will prolong substrate binding by DnaK to an extent that cell function is disrupted. GrpE is therefore essential in E. coli (Brehmer et al., 2004). However, unlike the bacterial DnaK, the eukaryotic Hsp70 has a considerable off-rate for ADP. In principle, it could perform its work in cells in a NEF-independent way. Thereby, nucleotide exchange is apparently not the rate-limiting step in eukaryotes. This is in sharp contrast to the essential role of GrpE for DnaK. However, eukaryotic Hsp70 can interact with nucleotide exchange factors to further accelerate the rate of nucleotide dissociation (Höhfeld et al., 1995).

A number of crystal structures of Hsp70-NEFs complexes has been determined (Harrison et al., 1997; Polier et al., 2008). All the NEFs appeared to trigger an outward rotation of subdomain IIB in the Hsp70 NBD, except for HspBP1. Consequently, the bound nucleotide can be released more easily. However, each family probably prefers a slight different mechanism.

Figure 7 shows an overview of the different forms of NEFs defined from different family. 


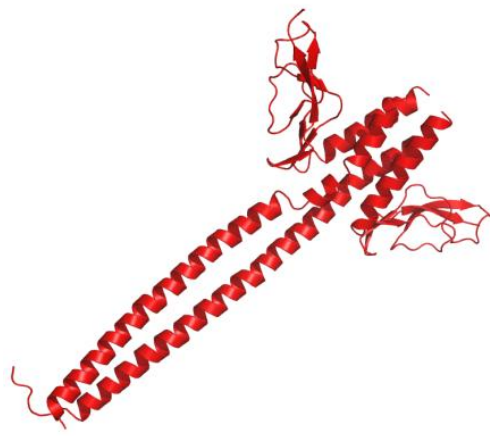

Family I

GrpE homologs
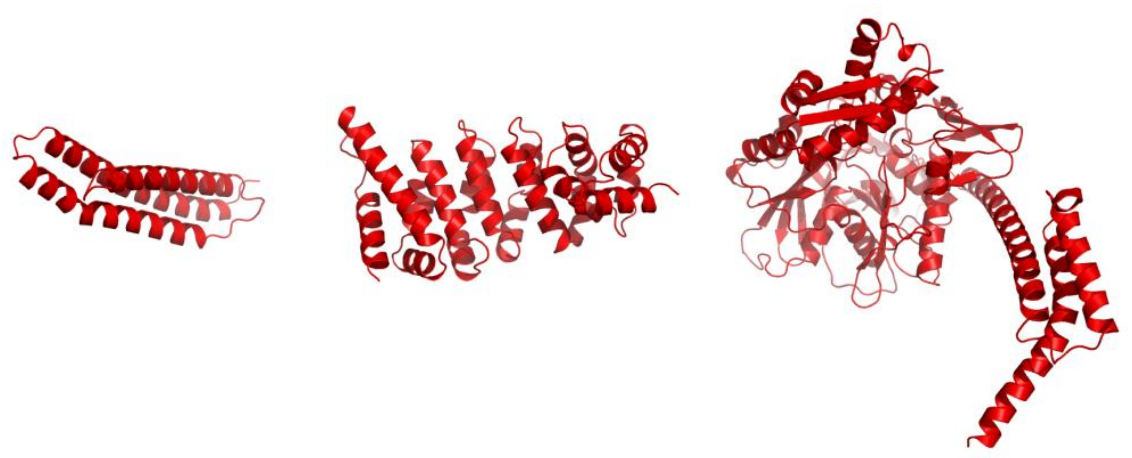

Family III

HspBP1
Family IV

Hsp110

Figure 7: Structures of the different NEFs shown in ribbon representation.

GrpE is the NEF of the E. coli homolog of Hsp70, DnaK. It forms a homodimer, which is mediated by the $\alpha$-helical domain. Form II, the BAG domain, is composed of three helices which are present in most BAG-domain proteins, except for BAG2. Family III shown here is the core domain of human HspBP1. It is an all- $\alpha$-helical protein. Sse1 of family IV is the yeast homolog of Hsp110, which is structurally highly similar as Hsp70, containing NBD, $\beta$ sandwich and three $\alpha$-helices domains. All structures are shown in ribbon representation and presented on the same scale. PDB accession numbers are as follows: 1DKG (Harrison et al., 1997), 1HX1 (Sondermann et al., 2001), 1XQS (Shomura et al., 2005) and 3D2F (Polier et al., 2008).

\subsubsection{Family I}

GrpE (GroP-like gene E) functions as a nucleotide exchange factor for bacterial Hsp70, DnaK. It is heat-inducible and essential in E. coli. GrpE eukaryotic homologs are found in mitochondria and chloroplasts cell organelles derived from bacteria. The yeast mitochondrial GrpE is named as Mge1, which is not heat-inducible in contrast to GrpE (Ikeda et al., 1994). Both share low sequence homology with only $34 \%$ identity and $57 \%$ similarity (Laloraya et al., 1994). Dimers of GrpE show an intriguing architecture with two almost parallel long $\alpha$-helices contributing to a four-helix bundle and two proximal $\beta$-sheet domains, which look like two wings (Fig. 7). In the co-crystal complex structure with DnaK, one monomer is bent towards DnaK, which leads to an asymmetric dimer appearance (Harrison et al., 1997).

\subsubsection{Family II}

There are six homologs in the family of BAG proteins in humans, named BAG1 to BAG6. All the homologs contain one BAG domain, except for BAG5, which is comprised of five putative 
BAG domains. In contrast to the $\alpha$ - $\beta$-dimer structure of GrpE, the BAG domain of BAG1 (Bcl2-associated athanogene) forms a three-helix bundle (Fig. 7). BAG-1 was initially thought as a cellular partner to bind the anti-apoptotic protein $\mathrm{Bcl}-2$ and to promote cell survival (Takayama et al., 1995). The six BAG proteins are modular multi-domain proteins and seem to recruit Hsp70 for specific functions, though they employ a rather similar way to induce conformational change on Hsp70. Besides the BAG domain, BAG1 isoforms also share an ubiquitin-like Ub1 domain which was implicated in proteasomal degradation, similar to the cochaperone Chip. BAG3 is involved in autophagy.

\subsubsection{Family III}

HspBP1 (Hsp70 binding protein 1) has another structural framework that was recruited for NEF function, a succession of armadillo repeats found in diverse structural contexts. HspBP1 is relatively abundant and it was initially described as an Hsp70-interacting protein that inhibited Hsp70-mediated protein refolding (Raynes and Guerriero, 1998). HspBP1 homologs represent a distinct class of conserved NEFs and relative abundant in cytosol, comparing to BAG domain proteins. The HspBP1 homolog in S. cerevisiae cytosol was named Fes1p. The structure of the core fragment of HspBP1 has an all- $\alpha$-helical structure containing four armadillo repeats (Fig. 7) (Shomura et al., 2005). In contrast to the other NEFs, HspBP1 binding to the NBD causes much more drastic structural changes. It was suggested that HspBP1 distorts the lobe I of Hsp70NBD and separates it from lobe II.

\subsubsection{Family IV}

The principal class of eukaryotic NEF as judged from its abundance appears to be Hsp110, which is predominantly located in cytosol and its homolog Grp170 in the ER-lumen. After Hsp70 and Hsp90, it is the third most abundant heat shock protein in most mammalian cell lines and tissues. There are three human Hsp110 homologs, Hsp105, Apg1 and Apg2, in mammalian cytosol. Two homologs, Sse1 and Sse2, are found in the cytosol of S. cerevisiae (Easton et al., 2000). They are highly homologous with $76 \%$ sequence identity. Both of them are upregulated upon stress. Sse1 is abundantly expressed under normal cellular conditions. Disrup- 
tion of Sse1 causes a growth defect and deletion of Sse 2 causes no distinct phenotype, however double deletion results in synthetic lethality (Mukai et al., 1993).

Interestingly, Hsp110 is a remote homolog of Hsp70 and shares the general domain composition with Hsp70. It consists of an NBD, a $\beta$-sandwich domain and a three-helix bundle domain (Fig. 7). The main difference with Hsp70 is the presence of an acidic insertion into the $\beta$ sandwich domain and an extended C-terminus. It was previously hypothesized that the Sse1ATP structure represents an evolutionary vestige of Hsp70-ATP (Liu and Hendrickson, 2007). Intriguingly, the recent available structure of DnaK-ATP showed a similar structure, confirming that Hsp70s and Hsp110 are homologs (Qi et al., 2013). The most remarkable difference between the DnaK-ATP and Sse1-ATP structures was the altered conformation of the $\beta$ sandwich and three-helix bundle domains.

\subsubsection{Cellular functions of NEFs}

Through their crucial role as nucleotide exchange factors, Hsp70 NEFs perform a wide variety of cellular functions, ranging from transcriptional regulation to the control of cell migration. In addition, some may participate in the targeting of substrates to the proteasome (Lüders et al., 2000). For the BAG-1 protein a role in coordinating chaperone and degradation pathways was suggested. The ER homolog of HspBP1 in humans, Sil1, has been revealed as an essential component of the endoplasmic-reticulum-associated protein degradation (ERAD) machinery and the unfolded protein response. Moreover, disruption of Sil1 leads to accumulation of misfolded protein in ER and nucleus, which causes a neurodegenerative disease, MarinescoSjögren syndrome (Anttonen et al., 2005; Senderek et al., 2005). Hsp110 is a distant Hsp70 homolog, possessing holdase activity, which can stabilize unfolded proteins and prevent aggregation. It was initially characterized as "holdase", as they can keep denatured protein in solution (Oh et al., 1997; Oh et al., 1999). In addition, it can bind to a misfolded protein, but alone lacks substrate refolding activity (Dragovic et al., 2006). Passive substrate binding has been attributed to Hsp110 which might be related to Hsp70-mediated folding (Polier et al., 2010). Furthermore, Hsp110s were found associated with amyloidogenic proteins and disease-related

protein aggregates such as from mutant superoxide dismutase 1 (SOD1) or tau (Eroglu et al., 2010; Olzscha et al., 2011; Yamashita et al., 2007). More recently, Hsp110 has been implicated 
to empower Hsp70-Hsp40 to efficiently resolubilize and reactivate substrate protein in vitro (Rampelt et al., 2012). Moreover, down-regulation of Hsp110 triggered formation of heat shock-induced luciferase aggregates in the model system C.elegans. This finding demonstrated that C. elegans unable to resolubilize luciferase aggregate in the absence of Hsp110, suggesting Hsp110 is an essential component of protein disaggregation machinery (Rampelt et al., 2012). In line with this finding, deletion of Hsp105 causes accumulation of hyper-phosphorylated tau and neurofibrillary tangles in mice (Eroglu et al., 2010). Taken together, the multiple functions of NEFs indicate the functional significance of NEFs in the Hsp70 molecular machinery.

\subsection{Hsp70 protein-protein network}

\subsubsection{Eukaryotic Hsp70 protein-protein network}

Hsp70 as a major chaperone in the mammalian cytosol and nucleus has been widely recognized not to act alone, but to cooperate with multiple cofactors and other chaperone systems to maintain a functional proteome (Buchner, 1999; Caplan, 1999; Frydman and Höhfeld, 1997). These cofactors do not only regulate the ATPase activity and substrate affinity of Hsp70, but also recruit Hsp70 to other chaperones and to components of the protein degradation machinery, thereby distributing functional proteins to functional complexes and organelles and damaged and misfolded proteins to proteolytic compartments. Figure 8 provides an overview of the main interactions of Hsp70 summarized by online database STRING (http://string-db.org/). 


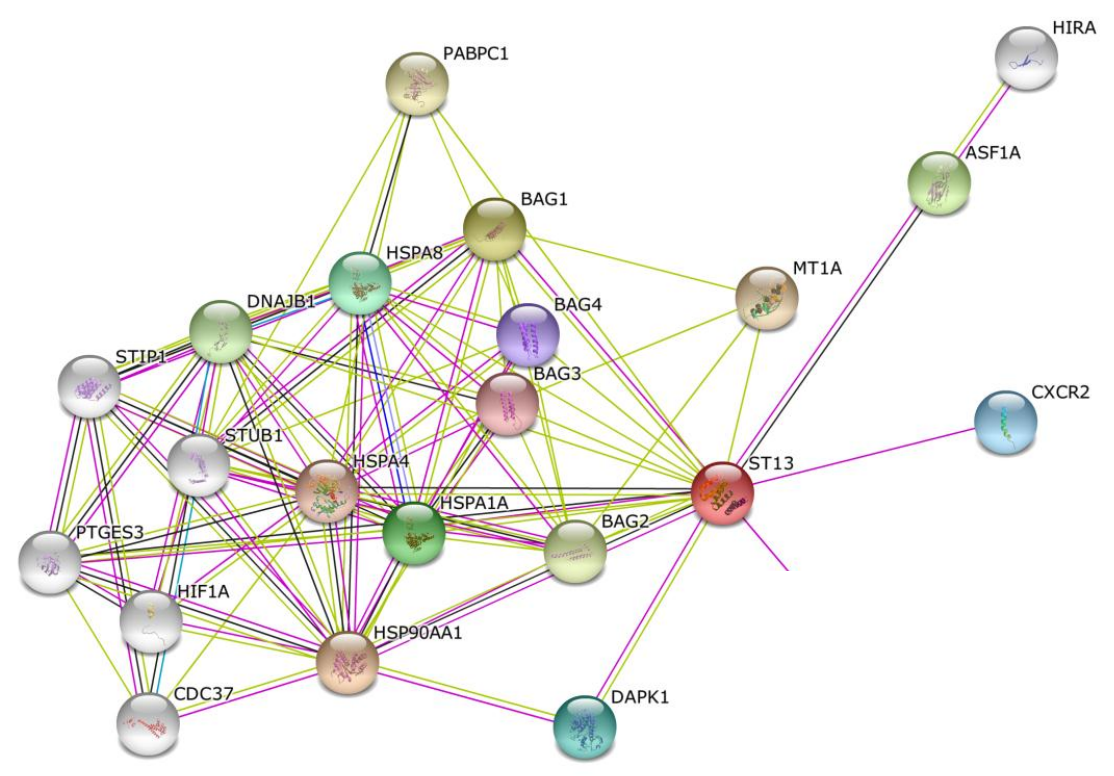

Figure 8: Overview of protein-protein interactions in the human Hs

The network was generated by database STRING. The interactions include direct and indirect associations.

For the cooperation of Hsp70 with the downstream Hsp90 system and the ubiquitin proteasome system (UPS), mainly three cofactors have been implicated in Figure 8: Hip (ST13), Hop (STIP1) and Chip (STUB1). In the following chapters, each of them is discussed in greater detail, but with special emphasis on Hip.

\subsubsection{Hsp70 interacting protein (Hip)}

Animals, plants and protozoa contain a conserved additional regulator of the Hsp70 reaction cycle, the cytosolic Hsp70 interacting protein (Hip). Alternative names for Hip are suppressor of tumorigenicity (ST13) and human p48. Human p48 shares over $91 \%$ identity with rat Hip. It is a highly conserved protein found in most eukaryotic lines, but absent in S. cerevisiae and other fungi (Appendix 8.2). The Hip concentration in reticulocyte lysate is $\sim 1 \mu \mathrm{M}$, similar to Hsc70. Hip is a dimeric protein of protomers with $\sim 43 \mathrm{kDa}$ molecular weight (human Hip). Hip was originally identified as a 368 residue protein binding specifically to the NBD of Hsp70 in the presence of $\mathrm{ADP}$ and participating in the regulation of eukaryotic Hsp70 protein (Höhfeld et al., 1995). Independently, Hip was identified as a component (p48) of a complex of a progesterone receptor folding intermediate, which also contained Hsp70 and Hsp90 (Smith et 
al., 1995). Hip was also found as interaction partner of the chemokine receptors C-X-C-motif chemokine receptors type 2 and 4 (CXCR2 and CXCR4), required for receptor internalization (Fan et al., 2002).

The existence of Hip suggests a regulatory mechanism in the eukaryotic cytosol distinct from bacterial DnaK. Hip was proposed to stimulate the chaperone activity of Hsp70 by preventing premature substrate release (Mayer and Bukau, 2005). Besides affecting the Hsp70 chaperone activities in vitro and in vivo, Hip possesses intrinsic molecular chaperone activity, as it binds specifically to unfolded proteins and prevents their aggregation (Höhfeld et al., 1995; Velten et al., 2002; Velten et al., 2000). Hip however cannot fold substrate proteins independently from Hsp70 and has no intrinsic ATPase activity (Bruce and Churchich, 1997; Höhfeld et al., 1995; Velten et al., 2000). Hip may be specifically recruited to Hsp70 by certain substrates. In addition, Hip was also proposed to compete with BAG1 for binding to the NBD of Hsp70, thereby preventing BAG1 stimulated ADP release (Höhfeld and Jentsch, 1997).

\subsubsection{Predicted domain structure of Hip}

Hip is thought to be composed of five domains, a dimerization domain at its N-terminus, followed by a tetratricopeptide repeats (TPR) domain flanked by highly negatively charged regions and a conserved region at its $\mathrm{C}$-terminus containing several GGMP peptide repeats and a DP domain. In the absence of structural data, most of these domains were proposed based on sequence alignment criteria, structural modelling and mutagenesis studies (Irmer and Höhfeld, 1997; Prapapanich et al., 1996b; Velten et al., 2000). The schematic representation of the domain structure of Hip is shown in Figure 9.

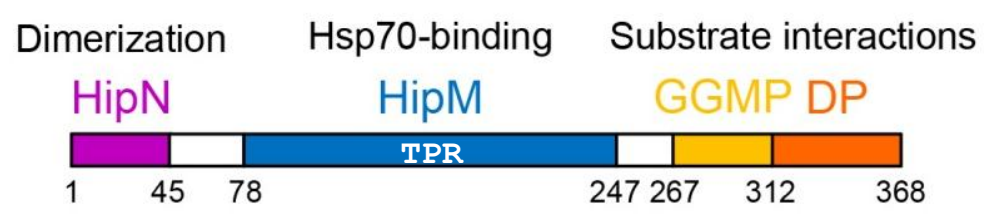

Figure 9: Overview of the domain structure of Hip. 
The residue numbers refer to Hip from Rattus norvegicus. The dimerization domain (HipN), Hsp70-binding TPR domain (HipM) and the DP domain are shown in purple, blue, and orange, respectively. White, acidic linker regions; Yellow, GGMP repeat segment.

\subsubsection{The $\mathrm{N}$-terminal dimerization domain of Hip}

Initially the number of Hip subunits in the Hip homo-oligomer was unclear (Höhfeld et al., 1995; Ziegelhoffer et al., 1996). Later Hip was unambiguously shown by a combination of size-exclusion chromatography and analytical ultracentrifugation to form elongated dimers (Velten et al., 2000). Deletion of 14 residues from the N-terminus of Hip resulted in the apparent loss of Hip homo-oligomerization. This indicates that dimerization was required for Hipassisted nuclear receptor maturation in vivo (Irmer and Höhfeld, 1997; Nelson et al., 2004; Nollen et al., 2001).

In the course of my master project, I cloned the conserved 44 residues at the $\mathrm{N}$-terminus of rat Hip, named Hip(1-44), and determined its crystal structure at 1.1 Å resolution (Zhuo Li, Master thesis). An exchange experiment using Hip(1-44) and full length Hip revealed slow exchange between the dimers (Figure 10C), suggesting stable dimerization on a molecular time scale (Zhuo Li, Master thesis).

A)

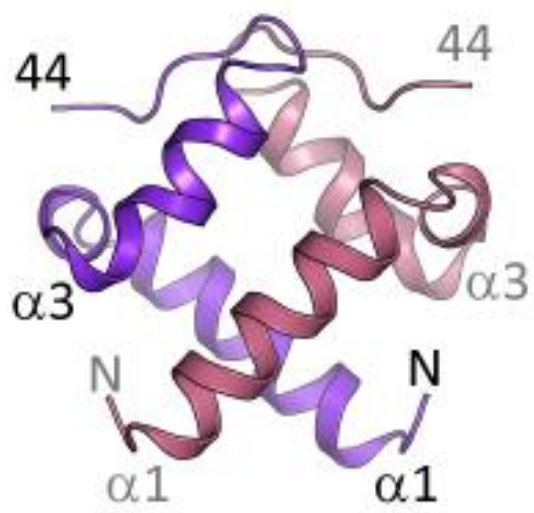


B)

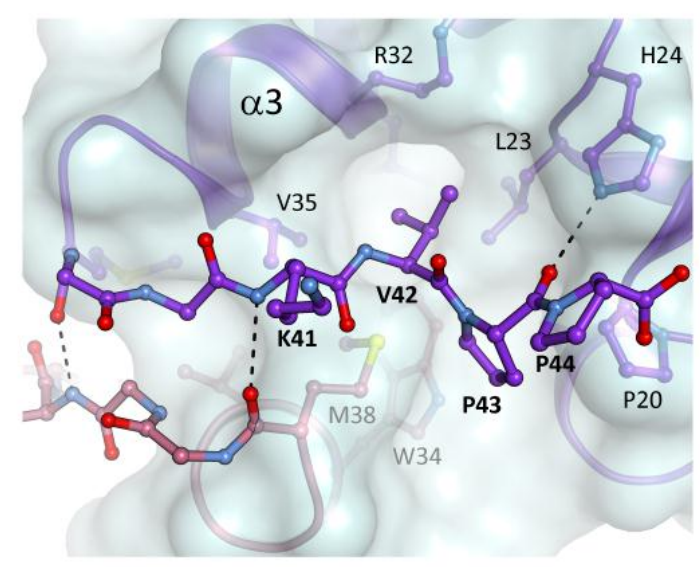

C)

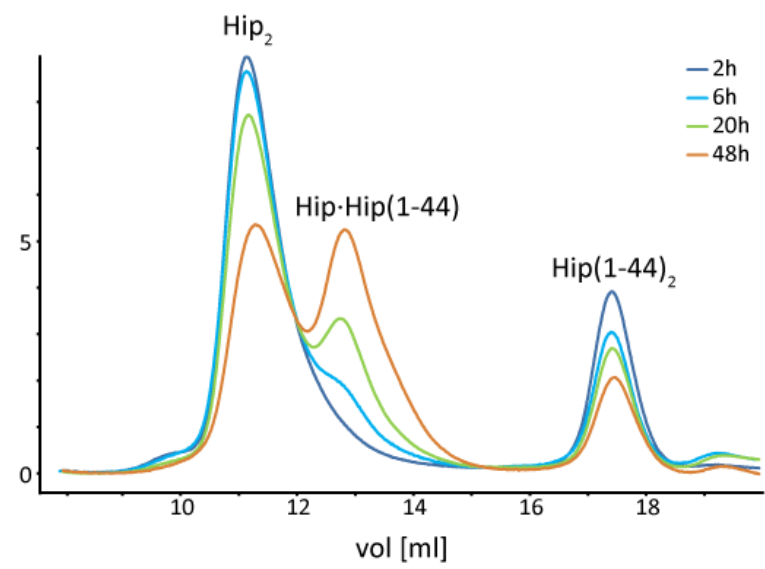

Figure 10: The dimerization domain of Hip.

A) Ribbon representation of the crystal structure of the dimerization domain of Hip. Protomers are shown in purple and salmon. Chain termini are indicated.

B) The dimer structure is stabilized by the contacts at the C-terminal end of the dimerization domain. The hydrogen bonds involved are indicated by dashed lines.

C) Slow subunit exchange between Hip dimers ( $\mathrm{Hip}_{2}$ and $\left.\mathrm{Hip}(1-44)_{2}\right)$ analyzed by size-exclusion chromatography. A stoichiometric mixture of Hip and $\mathrm{Hip}(1-44)$ was incubated at $20{ }^{\circ} \mathrm{C}$ and analyzed at the times indicated.

The structure showed a compact, $\alpha$-helical dimer with each subunit contributing three helices (Fig. 10A). Interestingly, the helices are oriented at nearly $90^{\circ}$ angles. This leads to a box-like parallel dimer structure, which is stabilized by van-der-Waals interactions of the side chains in the hydrophobic core. The C-terminal loops are stabilized by hydrogen bonds between the backbones at Lys41 and Met38 (Fig. 10B). No structural homologs were found in the Protein Data Bank with the DALI server. Similarly, no sequence homologs were found apart from sequences that were likely Hip homologs. This domain type might thus be unique to the Hip protein family.

To characterize the stability of the Hip dimer, the monomer exchange between $\mathrm{Hip}_{2}$ and Hip(1$44)_{2}$ at $20{ }^{\circ} \mathrm{C}$ was investigated over time by size-exclusion chromatography. During hours, the Hip-Hip(1-44) heterodimer complex band gradually increased, suggesting slow exchange (Fig. 10C). The estimated half-time of exchange is $\sim 6 \mathrm{~h}$. This indicates that Hip forms a constitutive dimer on a physiological timescale. 


\subsubsection{The TPR domain of Hip}

Tetratricopeptide repeat (TPR) domain is composed of TPR motif, which is composed of 34 amino acids (Lamb et al., 1995). Typically three successive TPR motifs and a C-terminal capping helix form a TPR domain. Specialized TPR domains recognizing the C-terminal EEVD motifs in Hsp70 and Hsp90 are present in their co-factors Hop, Chip, phosphoprotein phosphatase 5 (PP5), aryl hydrocarbon receptor interacting protein (AIP) and Hsp90-binding immunophilins Cyp-40, FK506-binding protein FKBP51 and FKBP52 (Ballinger et al., 1999; Buchner, 1999; Dolinski et al., 1998; Pratt and Toft, 1997; Scheufler et al., 2000).

Hip contains a predicted TPR domain between residues 113-214 (Höhfeld et al., 1995; Irmer and Höhfeld, 1997; Prapapanich et al., 1996b; Velten et al., 2002). Analysis of deletion constructs suggested that both the predicted TPR domain and the following positively charged region are required for efficient binding to the ATPase domain of Hsp70 (Prapapanich et al., 1996b). The TPR domain alone was not sufficient for binding to Hsp70 in the yeast two-hybrid system (Irmer and Höhfeld, 1997). The shortest construct displaying interaction with the ATPase domain of Hsp70 comprised residues 15-257 of Hip (Irmer and Höhfeld, 1997).

Truncation of the TPR domain from Hip unable it bind to Hsp70 (Prapapanich et al., 1996a), but its binding to CXCR2 was maintained and CXCR2-mediated mitogen-activated protein kinase activation can be preserved. However, overexpression of the TPR depletion mutant of Hip significantly attenuated the internalization of the chemokine receptors CXCR2 and CXCR4 (Fan et al., 2002).

\subsubsection{The GGMP repeat region}

Hip comprises seven imperfect Gly-Gly-(Met/Phe)-Pro (GGMP) tetrapeptide repeats between residues 278-311, and this motif is located close to the C-terminus of Hip together with an additional Sti1-related region (Höhfeld et al., 1995). It is highly conserved and is expanded to over 20 tandem repeats in a Plasmodium homolog of Hip (Uparanukraw et al., 1993). Similar repeats were also located in other chaperones, for example close to the peptide binding site of the C-terminus of Hsc70 as part of a regulatory motif (Höhfeld et al., 1995). Notably, inducible Hsp70 lacks this motif, as previously noted (Prapapanich et al., 1996a). It is interesting that Hip 
combines structural elements found in Hsc70, Hsc70 and Hsp90-associated co-chaperones. Because of its proline-rich nature, it was predicted that neither $\alpha$-helix nor $\beta$-sheet structure could be present in this region (Prapapanich et al., 1996a). The in vitro and in vivo studies suggested that this motif was not involved in regulating Hsp70 chaperone activity (Irmer and Höhfeld, 1997; Nollen et al., 2001). The exact function of the GGMP repeats in the interaction with Hsp70 or other cellular components is not yet understood.

\subsubsection{The DP domain of Hip}

The C-terminal region of Hip (residues 312-368) comprises two Asp-Pro (DP) repeat motifs with similarity to segments of the Hsp70-Hsp90 organizing protein (Hop) and Rad23. Little is known about how the DP region participates in co-chaperon function (Nelson et al., 2003).

The C-terminal domain of Hip was implicated in binding to the $\mathrm{C}$-X-C-motif chemokine receptors type 2 and 4 (CXCR2 and CXCR4). Truncation of the C-terminal 66 amino acids completely abolished the interaction of Hip with CXCR2 (Fan et al., 2002), suggesting substrate binding ability of C-terminus of Hip. In addition, it was previously found that mutants that destabilized the DP domain can stabilize interaction with Hsp70, while inhibiting PR assembly (Prapapanich et al., 1998). Moreover, a recent study identified Hip as a novel substrate of G protein-coupled receptor kinase 5 (GRK5), which recognizes Hip by a motif within the DP domain, residues 303-319, and phosphorylates Hip at Ser346 in vitro and in vivo (Barker and Benovic, 2011). GRK5 had previously not been implicated in signaling and trafficking of CXCR4. Phosphorylation of Hip by GRK5 was shown to play an essential role in modulating CXCR4 internalization.

\subsubsection{C-terminus of Hsc70 interacting protein (Chip)}

Aberrant proteins or protein aggregates destined for degradation need to be labeled with a multi-ubiquitin chain. The ubiquitination process is mediated by the cooperation of three enzymes: activator E1, ubiquitin conjugating E2 and ubiquitin ligase E3 enzyme. C-terminus of Hsc70 interacting protein, Chip, a $~ 35 \mathrm{kDa}$ protein, was the first known ubiquitin ligase that directly associated with a chaperone. Chip was initially identified in a screen for human TPR- 
containing protein (Ballinger et al., 1999). Chip is a dimeric protein with subunits containing TPR and U-box domains at N- and C-terminus, respectively. The TPR domain in Chip enables the binding to the C-terminal EEVD motifs of Hsp70 and Hsp90, and the U-box domain participates in the ubiquitin conjugation. Chip was shown to associate with Hsp70, block the ATPase cycle of Hsp70 and inhibit its ability to refold non-native protein (Ballinger et al., 1999). Similarly, Chip may abolish the cooperation of Hsp90 with other co-chaperones needed for productive chaperone function. However, based on the modular structure of Chip, it may also enable a direct link between Hsp70 and Hsp90, ubiquitinate the clients and target them to proteasome for degradation.

Chip was shown by isothermal titration calorimetry (ITC) to have a six-fold higher affinity for Hsp90 than Hsp70 in vitro (Stankiewicz et al., 2010). The same study also suggested that Chip preferentially ubiquitinated Hsp70-bound substrates, such as luciferase (Stankiewicz et al., 2010). Chip was however also shown to promote the degradation of glucocorticoid hormone receptor (GR) by eliciting release of the regulatory cofactor p23 from the ternary complex (Connell et al., 2001). In line with this finding, Chip targeted the degradation of the ubiquitinated Hsp90-associated immature cystic-fibrosis transmembrane-conductance regulator (CFTR) (Meacham et al., 2001). Importantly, overexpression of the U-box truncation fragment in Chip blocked CFTR ubiquitination, indicating the essential function of U-box domain for targeting substrate degradation (Meacham et al., 2001). In addition to CFTR, Chip has been shown to be involved in degradation of mutant superoxide dismutase 1 (SOD1), which is implicated in protein misfolded disease (Urushitani et al., 2004). In addition, Chip was implicated in mammalian longevity through defective protein control. A Chip-knockout mouse model exhibited a markedly reduced life span, along with accelerated age-related pathophysiological phenotypes (Min et al., 2008). Taken together, Chip appears to play a crucial role in maintaining protein quality control in cells by modulating the balance of folding and degradation of chaperone substrates.

\subsubsection{The Hsp70-Hsp90 organizing protein (Hop)}

The yeast homolog to Hop, Sti1, was initially identified in a genetic screen for proteins that mediate the heat-shock response in S. cerevisiae (Nicolet and Craig, 1989). Subsequently, in 
studies on the maturation of progesterone receptor (PR), mammalian Hop has been identified together with Hsp40, Hsp70 and Hip as an essential component for the recruitment of this steroid receptor to Hsp90 (Smith et al., 1993). Hop showed effective enhancement of the maturation of PR during in vitro reconstitution experiments. It is however not required for GR maturation in the same system (Morishima et al., 2000).

Hop is an abundant and highly conserved protein in eukaryotes. The homolog in S. cerevisiae is however not essential (Chang and Lindquist, 1994). Hop is a multi-domain adaptor protein that can bind Hsp70 and Hsp90 simultaneously. So far Hop has not been shown to act as a chaperone independently of Hsp70 and Hsp90 (Bose et al., 1996).

The various homologs of Hop are structurally characterized by the presence of three TPR domains, TPR1, TPR2A and TPR2B (residues 4-118, 223-352 and 353-477 in human Hop). The N-terminal TPR1 was shown to preferentially bind to the highly conserved EEVD motif at the C-terminus of Hsp70 (Scheufler et al., 2000). Interestingly, both TPR1 and TPR2B were identified to contribute to the Hsp70 interaction and they have redundant or overlapping functions in vivo (Flom et al., 2006). Conversely, TPR2A and TPR2B domains interact preferentially with the EEVD residues at the C-terminus of Hsp90 (Scheufler et al., 2000; Young et al., 1998). Notably, Hop contains two DP domains following TPR1 and TPR2B, called DP1 and DP2, respectively. Their sequences are $27 \%$ identical and they form the arrangement in TPR1-DP1TPR2A-TPR2B-DP2. Both of the two DP domains contribute to Hop functions (Carrigan et al., 2004; Chen et al., 1998), though one study has shown that DP2 domain is dispensable for the Hsp70 and Hsp90 interactions (Flom et al., 2007). Since Hop simultaneously interacts with Hsp70 and Hsp90 via separate TPR domains, Hop was postulated to be essential for in integrating Hsp70-Hsp90 interactions. This was demonstrated by using purified Hsp70 and Hsp90s in vitro, which associate with each other only when Hop was present (Chen et al., 1996). Thereby, it has been proposed that Hop plays a crucial role in the transfer of client proteins from Hsp70 to Hsp90. Surprisingly, Hop was also reported as an ADP/ATP exchange factor in the recycling of Hsp70 system (Gross and Hessefort, 1996). In line with this study, the yeast homolog Sti1 was found to stimulate ATPase activity of yeast Hsp70 Ssa1 by a factor of about 200, indicating that Sti1 is a potent novel effector for the Hsp70 ATPase activity (Wegele et al., 2003). Furthermore, Sti1 binds to the N-and C-terminal part of Hsp90 and prevents the N-terminal dimerization reaction that is required for efficient ATP hydrolysis by Hsp90. Therefore, we can 
surmise that Hop might work as a functional homolog to GrpE in the eukaryotic cytosol, promoting the dissociation of the substrate from Hsp70 for the efficient transfer to downstream Hsp90 chaperone system.

\subsubsection{Similarities between Hip and Hop}

Both Hip and Hop contain TPR domains, which mediate binding to chaperones. In addition, they also share DP motifs at their C-termini (Höhfeld et al., 1995; Nelson et al., 2003). Figure 11 shows a comparison of the domain structure. The first DP unit between Hip and Hop has the same sequence DPEV, but the second, DPAM, is slightly different in Hop. Interestingly, the combination of TPR and DP domains can only be found in Hip and Hop homologs. Besides sharing TPR and DP domains, both contain highly charged regions. Both the sequences at the end of the DP domain of Hop and between the TPR and DP domains of Hip show a preference for the residues such as D, E, K, Q, S and T.

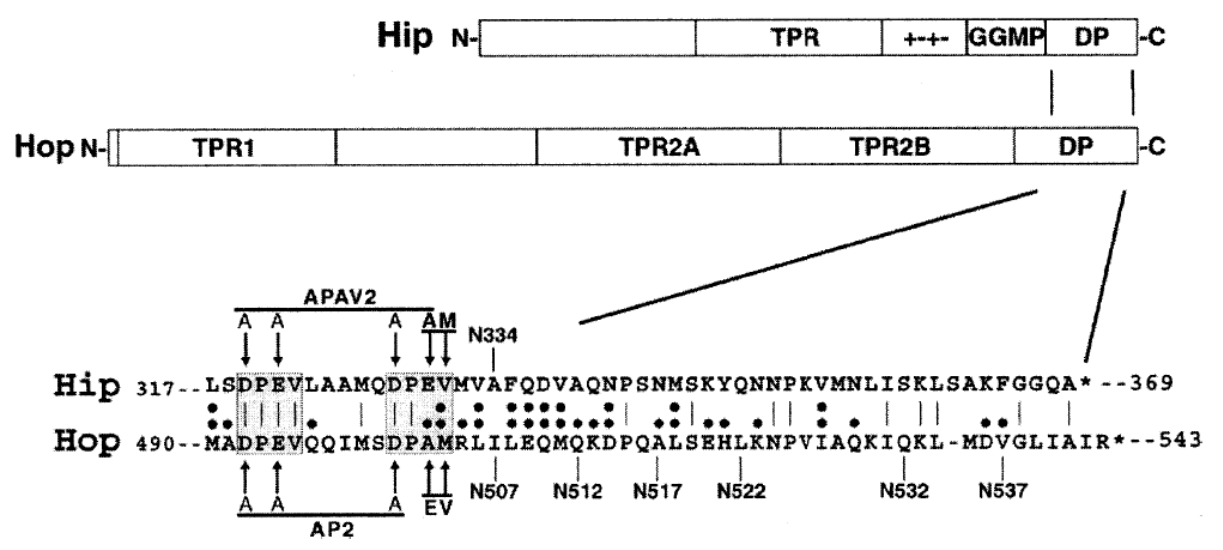

Figure 11: Diagram representation of Hip and Hop domains and shared C-terminal sequences.

Though the respective DP regions of Hip and Hop share sequence similarity, a functional defect in Hop was observed upon introduction of the double-point mutation DPAM to DPEV in the second DP motif of Hop, whereas the corresponding mutation in Hip maintains its function (Fig. 11) (Nelson et al., 2003). Therefore, they are not functionally equivalent. Moreover, both of them bind to Hsp70 but through distinct binding sites. For Hip binding, the N-terminal ATPase domain of Hsp70 is sufficient. Conversely, Hop recognizes the C-terminus of Hsc70 
(Demand et al., 1998). Notably, both of Hip and Hop are involved in the steroid receptor complex maturation. In Hop, truncation of the entire DP region or DP point mutants blocked Hop binding to Hsp70 and assembly into the progesterone receptor (PR) complex (Chen and Smith, 1998). In Hip, DP mutants bind to Hsp70 more stably than wild type Hip, but block recruitment of Hop and Hsp90 to the receptor complex (Prapapanich et al., 1998).

\subsection{Role of Hip in cellular protein quality control}

Hip has been shown to be important for stabilizing Hsp70 in the ADP-bound state (Höhfeld et al., 1995). It was implicated in progesterone receptor (PR) pathway and presumably mediates substrate binding to Hsp70 in early receptor complexes (Prapapanich et al., 1996a). In collaboration with Hsp70, Hip prevents the formation of large cytotoxic aggregates of $\alpha$-Synuclein and a polyQ-expanded mutant of androgen receptor (AR), hallmarks of Parkinson's disease and spinobulbar muscular atrophy (SBMA) (Kennedy's disease), respectively (Howarth et al., 2009; Roodveldt et al., 2009). Recently, Hip was also implicated in the ubiquitylation and proteasomal degradation of neuronal nitric oxide synthase and polyQ androgen receptor (Wang et al., 2013). Notably, a similar effect in promoting the degradation of polyQ androgen receptor and of hyper-phosphorylated Tau was achieved by the Hsp70-binding compounds MKT-077 and YM-1. These chemically related compounds stabilize Hsp70-substrate complexes and have been described as mimics of Hip function (Rousaki et al., 2011; Wang et al., 2013).

\subsubsection{Regulation of progesterone receptor maturation}

Steroid receptors are rapidly turned over in cells. In the absence of hormone, the receptor remains inactive. Upon hormone binding to the receptor, the complex disassembles, leading to release of the active receptor. The activation is mediated by intricate chaperone machinery in the cytosol. Hip was found to associate with Hsp70 in the ADP-bound state. It was suggested that Hip might contribute to the interaction of Hsc70 with various target proteins (Höhfeld et al., 1995). In addition, Hip was noted as a transient component during the cell-free assembly of progesterone receptor complex (Smith et al., 1995). Thereby, it was proposed to be an additional accessory factor paralleling Hsp70 in the assembly process. The identification of Hip as a 
component of the hormone receptor complex provides the possibility to understand the interactions between Hip, Hsp70 and Hsp90 in vivo, since all of them are components of the same multi-protein complex (Smith, 1993; Smith et al., 1995). Hsp90 and its co-factors, which are essential for hormone binding and the activation of receptor, were suggested to stabilize the receptor in the proper hormone binding conformation.

Therefore, the presence of Hip in the progesterone receptor complex provides us with an example for the intracellular cooperation between the molecular chaperones. Especially, Hip might works as a signaling protein to link Hsp70 with downstream Hsp90.

\subsubsection{Aggregation prevention in models of neurodegenerative disease}

Protein misfolding and aberrant self-assembly may cause cell toxicity, resulting in protein conformational disorders and neurodegeneration. Many neurodegenerative diseases such as Alzheimer's (AD), Parkinson's (PD) and Huntington's diseases (HD) are characterized by the deposition of insoluble protein inclusions (Chiti and Dobson, 2006). The role of small molecular co-chaperones in facilitating the removal of protein inclusions has been studied (Jana and Nukina, 2005).

A)
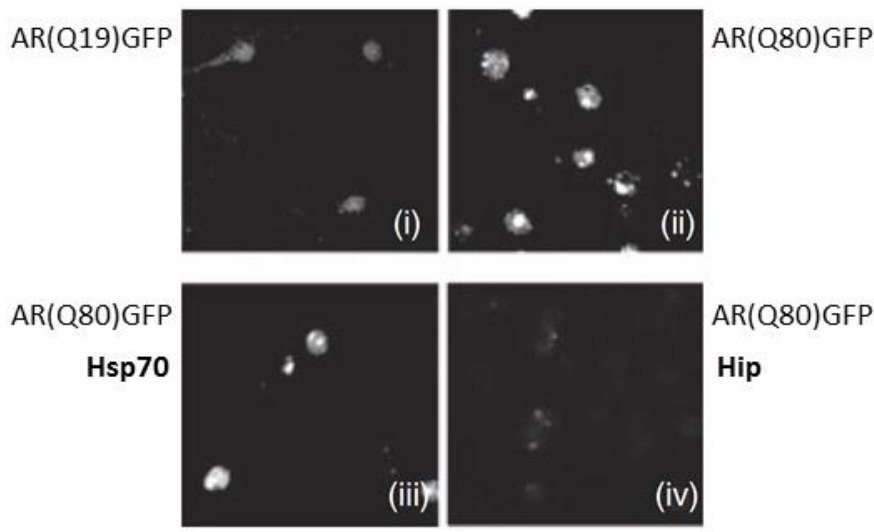

AR(Q80)GFP 
B)
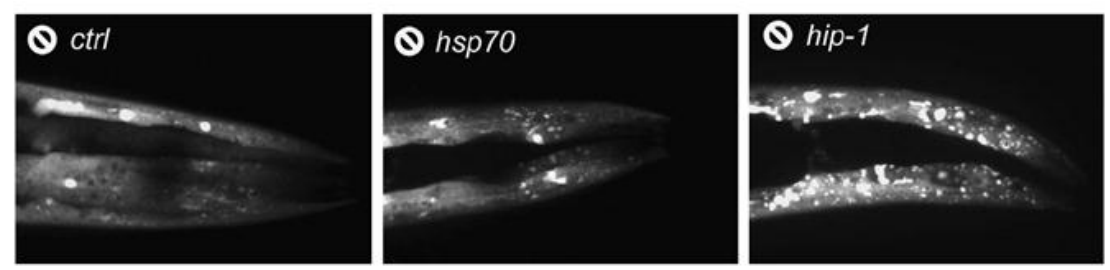

Figure 12: Suppression of protein deposit formation by Hip in vivo.

A) Cortical neurons were transduced with vectors expressing AR(Q19)GFP and AR(Q80)GFP fusion proteins with or without presences of additional chaperones. (i) vectors expressing AR(Q19)GFP; (ii) vectors expressing AR(Q80)GFP; (iii) AR(Q80)GFP in presence of Hsp70; (iv) AR(Q80)GFP in presence of Hip (Howarth et al., 2009).

B) $\alpha$-Syn inclusion formation in the head region of transgenic $C$. elegans worms. $\alpha$-Syn-YFP transgenic C.elegans were fed with bacteria expressing either empty vector (L4440), RNAi for knockdown of Hsp70 and knockdown of Hip. Quantification of the inclusion formation was performed in age-synchronized young adult C.elegans, after feeding RNAi bacteria for two generations (Roodveldt et al., 2009).

The effect of overexpressing Hip was initially investigated in a cellular polyglutamine (polyQ) model of spinal and bulbar muscular atrophy (SBMA) and Huntington's diseases (Howarth et al., 2009). Overexpression of Hip alone significantly reduced inclusion body formation (Fig. 12A). In addition, Parkinson's disease (PD) patients had consistently low Hip expression levels in their blood relative to healthy controls, even in the early stages of the disease (Scherzer et al., 2007). Intra-neuronal inclusions in the brains of PD patients known as Lewy bodies contain a large proportion of aggregated $\alpha$-Synuclein. The effect of Hip on $\alpha$-Synuclein aggregation was investigated in vitro and the C. elegans model system (Roodveldt et al., 2009). In the study, addition of Hip to Hsp70 in the presence of ATP efficiently suppressed $\alpha$-Synuclein fibrils' formation in vitro and no fibrils can be observed under transmission electron microscopy (TEM). Importantly, this anti-amyloidogenic activity of Hip is mediated by Hsp70, as overexpression of Hip alone cannot decrease the formation of $\alpha$-Synuclein fibrils. Depletion of Hsp70 by RNA interference caused only a non-significant increase of $\alpha$-Synuclein-YFP inclusions in transgenic worms, whereas knockdown of Hip significantly caused increased inclusion formation, suggesting an essential function of Hip in suppressing $\alpha$-Syn aggregation (Fig. 12B). It can be therefore postulated that depletion of Hip might impair the ability of proteins to resist aggregation and that Hip is required to maintain protein homeostasis. 


\subsubsection{Hip promotes degradation of misfolded protein}

Hip preferentially binds to Hsp70 in the ADP-bound state. Interestingly, a small molecule functional homolog of Hip named MKT-077 was identified, which also binds to the ADP-bound state of Hsp70, but not ATP state (Rousaki et al., 2011).

A)

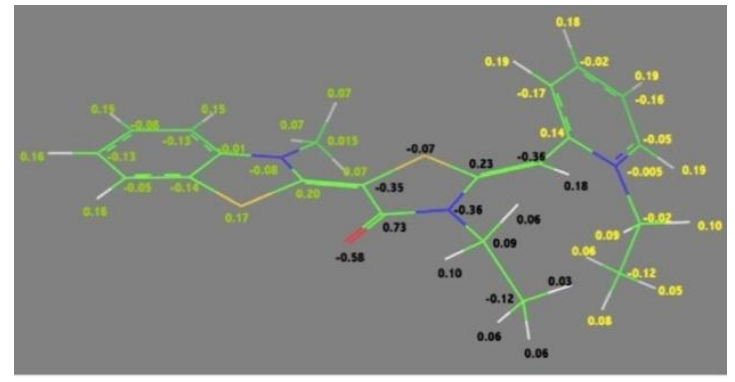

B)

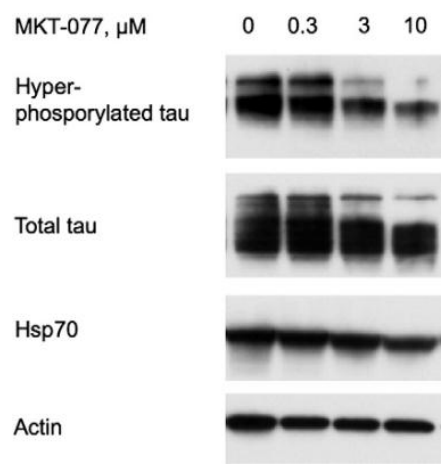

Figure 13: MKT-077, a compound mimicking Hip.

A) The molecular structure of MKT-077, based on quantum mechanical calculations.

B) MKT-077 promotes the clearance of hyper-phosphorylated tau in HeLa C3 cell line in a dose-dependent manner. SDS-PAGE analysis was performed after 24h incubation (Pitsis and Visouli, 2011).

MKT-077 is a rhodacyanine dye analog with the systematic name 1-ethyl-2-[[3-ethyl-5-(3methylbenzothiazolin-2-yliden)]-4-oxothiazolidin-2-ylidenemethyl] pyridinium chloride (Fig. 13A). MKT-077 has been identified initially as potent anticancer compound that interacted with mtHsp70 in pull-down studies (Deocaris et al., 2007; Wadhwa et al., 2000). Later MKT077 was proposed to act as an "allosteric drug", which blocks the cycle of cytosolic Hsp70 through stabilization of the ADP state (Rousaki et al., 2011). The $\mathrm{K}_{\mathrm{D}}$ value for the complex of MKT-077 and the ADP-bound NBD of Hsp70 was estimated to be in the range of $1-10 \mu \mathrm{M}$ by NMR titration. Selectivity towards the presumed binding site, a negatively charged pocket 
between subdomains IA and IIA of Hsp70 NBD, might be driven by the cationic character of MKT-077 (Rousaki et al., 2011). This location is close to the nucleotide binding site, but not identical to it.

In preclinical studies, MKT-077 was found to inhibit proliferation of multiple human cancer cell lines, including colon, breast carcinoma cells and showed no toxicity against normal kidney cells (Koya et al., 1996). However, because of renal toxicity in the clinical Phase I trial against solid tumors, testing of MKT-077 was stopped.

One of the hallmarks of Alzheimer's disease is the accumulation of hyper-phosphorylated Tau in neurons besides extracellular amyloid- $\beta$ deposits. Interestingly, MKT-077 promoted the clearance of hyper-phosphorylated Tau in a model of Alzheimer's disease, suggesting a potential link from Hip to the cellular degradation machinery (Fig. 13B) (Rousaki et al., 2011). Indeed, Hip overexpression facilitated ubiquitination and promoted proteasomal degradation of neuronal nitric oxide synthase (nNOS) and polyQ androgen receptor (AR) in cultured cells (Wang et al., 2013). A similar effect of increased ubiquitination and degradation of client proteins was observed with the compound YM-1, a stable and soluble derivative of MKT-077. As predicted, this compound also promoted binding of Hsp70 to denatured luciferase.

\subsection{Aim of study}

It had been suggested that Hsp70 interacting protein, Hip delays substrate release from Hsp70 by slowing the dissociation of Hsp70-bound ADP. However, the physiological role of Hip and its interplay with other Hsp70 co-factors were far from clear. Despite considerable achievements in understanding the Hip structure and its interaction with Hsp70, the exact threedimensional structure of Hip remained a mystery. The exact and detailed structural mechanism by which Hip regulates Hsp70 was unknown. Such data could provide the basis for rational drug design and novel therapeutic interventions.

Based on these considerations, this work focuses on a detailed structural and functional analysis of Hip. Our aim was to determine the crystal structures of Hip and the Hip:Hsp70 complex. To achieve our goal, we first delineated the probable domain boundaries in rat Hip from sequence alignment, which guided the design of various Hip constructs. Functional analyses by isother- 
mal titration calorimetry (ITC) and MABA-ADP dissociation assay identified the essential Hsp70 binding domain of Hip. With the information provided by the functional assays, the crystal structures of the middle domain of Hip (HipM) and the Hip:Hsp70 protein core complex were successfully determined. Subsequently, a detailed characterization of Hip:Hsp70 complex based on structure-guided site-directed mutagenesis of Hip was undertaken. These experiments characterized the Hip interface with Hsp70 and the structural requirement for the stabilization and regulation of Hsp70. Comparison of the Hip-Hsp70 and NEF-Hsp70 complex structures suggested Hip and NEF binding to Hsp70 are mutually exclusive. Thus, the association of Hip with Hsp70-substrate complexes attenuates their active cycling. Finally, the functional interaction between Hip and Hsp70 was probed in vivo, using the maturation of glucocorticoid receptor as a read-out.

In summary, the structural and biochemical analyses performed in this study were designed to provide a mechanistic model for the cooperation of Hip with Hsp70 in protein folding and aggregation prevention. 


\section{Materials and Methods}

\subsection{Chemicals}

Unless otherwise stated, chemicals were of pro analysis quality and purchased from Sigma-

Aldrich (Steinheim, Germany) or Merck (Darmstadt, Germany).

\begin{tabular}{ll}
\hline Suppliers & Chemicals \\
\hline Applied Biosystems (Foster, USA) & Gal-Screen chemiluminescence reagent \\
Biomol (Hamburg, Germany) & HEPES \\
Biozym (Hessisch Oldendorf, Germany) & Biozym LE Agarose \\
Clontech (Heidelberg, Germany) & Herring testis carrier DNA \\
Difco (Heidelberg, Germany) & Bacto Agar \\
& Bacto Tryptone \\
& Bacto peptone \\
& Bacto Yeast Extract \\
& Bacto Yeast Nitrogen Base \\
Fermentas (St. Leon-Rot, Germany) & GeneRuler 100 b und 1 kb DNA Ladder Page \\
Ruler Protein Ladder \\
PageRuler Prestained Protein Ladder \\
Fluka (Deisenhofen, Germany) & Polyethylene glycols of different molecular \\
& weights \\
Interchim (Montluçon, France) & Sodium cacodylate \\
Hampton Research (Aliso Viejo, USA) & Chloramphenicol \\
& MES \\
& Index Screen \\
& (MABA-ATP) \\
\hline
\end{tabular}




\begin{tabular}{|c|c|}
\hline \multirow[t]{3}{*}{ Invitrogen (Karlsruhe, Germany) } & pProEx-HtA, pProEx-HtB \\
\hline & protein marker for SDS-PAGE \\
\hline & SYBR Safe DNA gel strain \\
\hline J.M. Gabler Saliter GmbH \& Co.KG & Skimmed milk powder \\
\hline \multicolumn{2}{|l|}{ (Obergünzburg, Germany) } \\
\hline \multirow[t]{2}{*}{ Metabion (Martinsried, Germany) } & $\mathrm{dNTP}$ \\
\hline & Oligonucleotides (Primers) \\
\hline New England Biolabs (Frankfurt am Main, & BSA \\
\hline \multirow[t]{2}{*}{ Germany) } & Restriction endonucleases (Enzyme) \\
\hline & T4 DNA ligase (Enzyme) \\
\hline \multirow[t]{4}{*}{ Promega (Mannheim, Germany) } & Pfu polymerase (Enzyme) \\
\hline & Wizard Plus SV Miniprep DNA Purification \\
\hline & System \\
\hline & Wizard SV Gel and PCR Clean-Up System \\
\hline \multirow[t]{2}{*}{ Qiagen (Hilden, Germany) } & Qiagen Plasmid Midi Kit \\
\hline & Qiagen Plasmid Maxi Kit \\
\hline \multirow[t]{5}{*}{ Roche (Basel, Switzerland) } & DTT \\
\hline & EDTA-free Complete protease inhibitor cocktail \\
\hline & Hexokinase (Enzyme) \\
\hline & Proteinase K (Enzyme) \\
\hline & Shrimp alkaline phohsphatase (Enzyme) \\
\hline \multirow[t]{3}{*}{ Roth (Karlsruhe, Germany) } & Ampicillin \\
\hline & IPTG \\
\hline & $\mathrm{KCl}$ \\
\hline \multirow[t]{4}{*}{ Serva (Heidelberg, Germany) } & Acrylamide-Bis \\
\hline & Coomassie Blue R250 \\
\hline & PMSF \\
\hline & SDS \\
\hline
\end{tabular}




\subsection{Enzyme and antibodies}

\begin{tabular}{ll}
\hline Suppliers & Chemicals \\
\hline Fermentas (St. Leon-Rot, Germany) & Restriction enzyme EheI \\
New England Biolabs (Frankfurt am Main, & Restriction enzymes \\
Germany) & T4 ligase \\
Novagen /ToyoBo (Darmstadt, Germany) & KOD Hot Start DNA polymerasae \\
Promega (Mannheim, Germany) & Pfu polymerase \\
& T4 ligase \\
Department of Cellular Biochemistry, MPI & Rat polyclonal Anti-Hip, second bleed- \\
(Martinsried, Germany) & ing, dilution 1:1000 \\
Invitrogen (Karlsruhe, Germany) & Mouse polyclonal anti-PGK, dilution \\
& $1: 5000$ \\
Invitrogen (Karlsruhe, Germany) & Mouse monoclonal anti-Hsc70, dilution \\
& $1: 2500$ \\
Roche (Mannheim, Germany) & Mouse monoclonal anti-GFP, dilution \\
Sigma-Aldrich (Steinheim, Germany) & $1: 1000$ \\
\hline
\end{tabular}

Table 2: Enzyme and antibodies

\subsection{Strains}

\begin{tabular}{ll}
\hline Suppliers & Strains \\
\hline Novagen (Darmstadt, Germany) & E. coli DH5 $\alpha$ \\
Stratagene (Heidelberg, Germany) & E. coli BL21 (DE3) \\
Stratagene (Heidelberg, Germany) & E. coli BL21 (DE3) codon plus RIL \\
M.B. Cox (University of Texas, El Paso, & S. cerevisiae DSY-1100 \\
Texas, USA) & \\
\hline
\end{tabular}

Table 3: Strains 


\subsection{Instruments}

\begin{tabular}{|c|c|}
\hline Suppliers & Reagents \\
\hline Agilent Technologies (Santa Clara, USA) & Variable wavelength detector Agilent 1100 series \\
\hline Applied Photophysics (Surrey, UK) & SX. $18 \mathrm{~V}$ stopped-flow instrument \\
\hline Bachofer (Reutlingen, Germany) & Hybridization oven \\
\hline Beckman Coulter GmbH (Krefeld, & Centrifuges (GS-6R, Allegra-6R, Avanti J-25 \\
\hline \multirow[t]{5}{*}{ Germany) } & with rotors JKA 10.500 and JA 25.50, J6-MI \\
\hline & with rotor JS 4.2) \\
\hline & DU 640 UV/VIS Spectrophotometer \\
\hline & DU 800 UV/VIS Spectrophotometer \\
\hline & Optima LE $80 \mathrm{~K}$ ultracentrifuge with rotor $45 \mathrm{Ti}$ \\
\hline Bibby Scientific (Stone, UK) & Stuart rotating wheel \\
\hline Biometra (Göttingen, Germany) & PCR thermocycler \\
\hline \multirow[t]{5}{*}{ Bio-Rad (München, Germany) } & Chemidox XRS \\
\hline & Horizontal agarose gel electrophoresis \\
\hline & Mini Protean II electrophoresis cell \\
\hline & Power Pac 300 \\
\hline & (Wide) Mini-SUB CELL GT \\
\hline Biospec (Bartlesville, USA) & Bead Beater \\
\hline \multirow[t]{3}{*}{ Eppendorf (Hamburg, Germany) } & Centrifuges (5415D and 5417R) \\
\hline & Pipettes $(2,10,20,100,200,1000 \mu \mathrm{l})$ \\
\hline & Thermomixer comfort \\
\hline Fischer Scientific (Schwerte, Germany) & Accumet Basic $\mathrm{pH}$ meter \\
\hline \multirow[t]{3}{*}{ Fuji/Raytest (Straubenhardt, Germany) } & Fuji-LAS3000 luminescence and densitometry \\
\hline & system with Image Reader LAS-3000 \\
\hline & Gel imaging software: AIDA v.3.5.0 \\
\hline \multirow[t]{4}{*}{ GE-Healthcare (München, Germany) } & Äkta FPLC \\
\hline & Äkta Purifier/Explorer \\
\hline & Electrophoresis Power Supply - EPS600 \\
\hline & Ettan LC \\
\hline
\end{tabular}




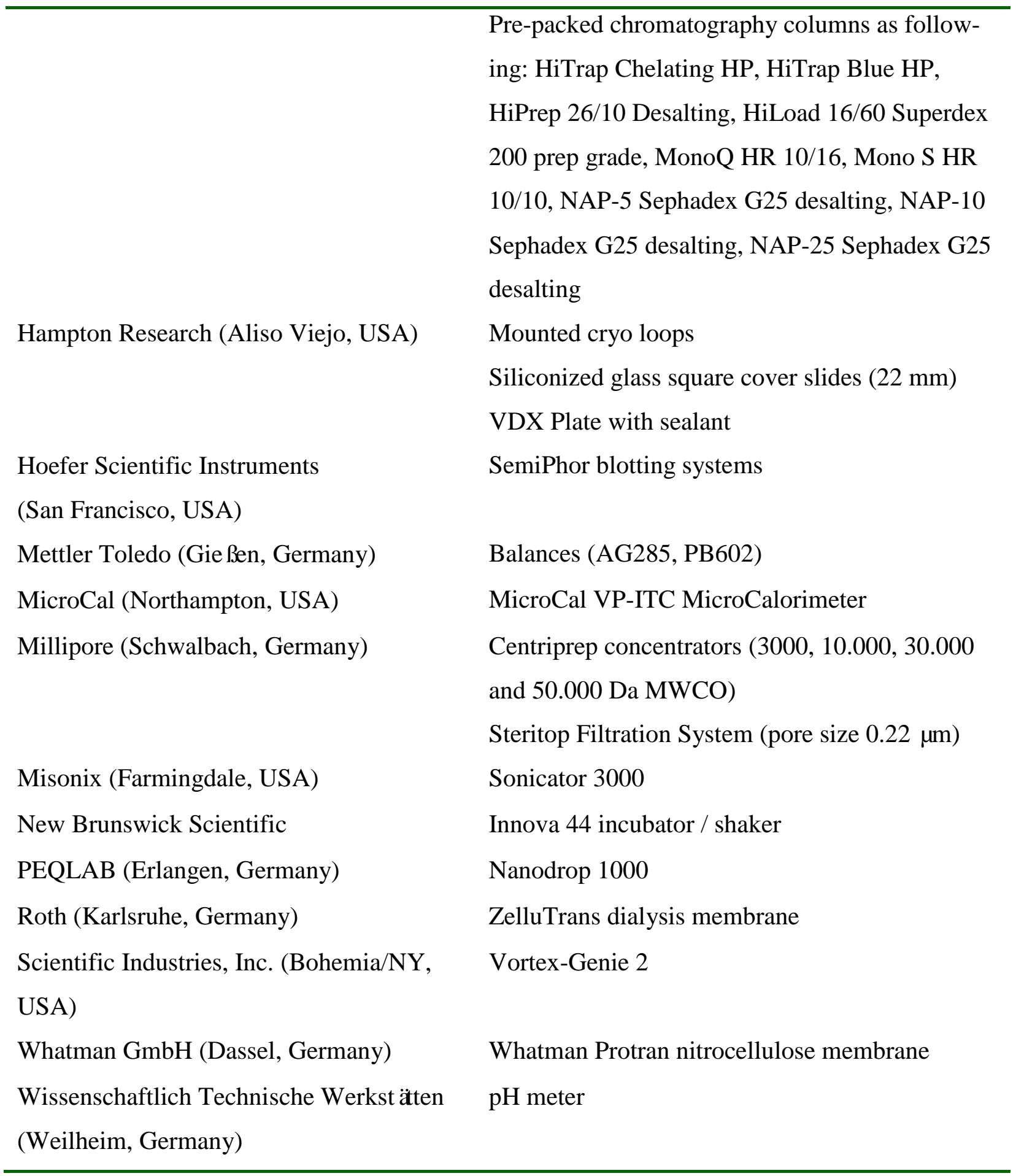

Table 4: Instruments 


\subsection{Media and buffers}

Media were prepared with deionized water and subsequently autoclaved. M9 minimal media was prepared with autoclaved water or sterile filtered ingredients.

\begin{tabular}{|c|c|}
\hline Media & Reagents \\
\hline \multirow[t]{5}{*}{ LB medium: } & $10 \mathrm{~g} / \mathrm{l}$ Bacto peptone \\
\hline & $5 \mathrm{~g} / 1$ Bacto Yeast Extract \\
\hline & $10 \mathrm{~g} / \mathrm{l} \mathrm{NaCl}$ \\
\hline & Adjust pH 7.0 with $5 \mathrm{M} \mathrm{NaOH}(\sim 0.7 \mathrm{ml} 5 \mathrm{M} \mathrm{NaOH} /$ liter$)$ \\
\hline & (15 g/l agar for solid medium) \\
\hline \multirow[t]{12}{*}{$\mathrm{SC}$ medium } & $6.7 \mathrm{~g} / \mathrm{l}$ Bacto Yeast Nitrogen Base \\
\hline & $2 \mathrm{~g} / \mathrm{l}$ drop-out mix \\
\hline & Adjust pH 5.6 with $5 \mathrm{M} \mathrm{KOH}$, autoclave \\
\hline & $40 \mathrm{ml} 50 \%(\mathrm{w} / \mathrm{v})$ glucose (sterile-filtered) \\
\hline & If required, the following amino acids were added in, which \\
\hline & were prepared in solution. \\
\hline & $55.3 \mathrm{mg} / \mathrm{l}$ adenine (autoclaved) \\
\hline & $62.9 \mathrm{mg} / \mathrm{l} \mathrm{L}$-histidine (autoclaved) \\
\hline & $22.4 \mathrm{mg} / \mathrm{l}$ uracil (autoclaved) \\
\hline & 81.7 mg/l L-tryptophan (sterile-filtered) \\
\hline & 182.6 mg/l L-lysine (autoclaved) \\
\hline & 219 mg/ml L-leucine (autoclaved) \\
\hline \multirow[t]{5}{*}{ SC plates: } & $20 \mathrm{~g} / \mathrm{l}$ Agar solution and SC medium (Bacto Yeast Nitrogen \\
\hline & Base/drop out mix) were autoclaved separately, as the low \\
\hline & pH of SC medium will cause acid hydrolysis of the agar. \\
\hline & After autoclaving, the agar solution was mixed with SC \\
\hline & medium. \\
\hline
\end{tabular}

Table 5: Media 


\begin{tabular}{|c|c|}
\hline Buffers & Reagents \\
\hline \multirow[t]{2}{*}{6 x DNA-loading buffer } & $0.25 \%(\mathrm{w} / \mathrm{v})$ bromophenol blue \\
\hline & $0.25 \%(w / v)$ xylene cyanol FF \\
\hline \multirow[t]{3}{*}{ Coomassie staining solution } & 0.1 (w/v) Serva Coomassie Blue R250 \\
\hline & $40 \%(\mathrm{v} / \mathrm{v})$ Ethanol \\
\hline & $10 \%(\mathrm{v} / \mathrm{v})$ Acetic acid \\
\hline \multirow[t]{2}{*}{ Coomassie destaining solution } & $10 \%(\mathrm{v} / \mathrm{v})$ Ethanol \\
\hline & $10 \%(\mathrm{v} / \mathrm{v})$ Acetic acid \\
\hline \multirow[t]{5}{*}{$2 \times$ SDS-loading buffer } & 100 mM Tris-HCl pH 6.8 \\
\hline & $4 \%(w / v)$ SDS \\
\hline & $200 \mathrm{mM}$ DTT \\
\hline & $20 \%(\mathrm{v} / \mathrm{v})$ glycerol \\
\hline & $0.2 \%(w / v)$ bromphenolblue \\
\hline \multirow[t]{3}{*}{$10 \mathrm{x}$ SDS-running buffer } & $3.02 \%(\mathrm{w} / \mathrm{v})$ Tris \\
\hline & $14.4 \%(\mathrm{w} / \mathrm{v})$ glycine \\
\hline & $1 \%(\mathrm{w} / \mathrm{v}) \mathrm{SDS}$ \\
\hline \multirow[t]{2}{*}{ Ponceau S stain } & $0.2 \%(\mathrm{w} / \mathrm{v})$ Ponceau S \\
\hline & $3 \%(\mathrm{v} / \mathrm{v})$ trichloro-acetic acid \\
\hline \multirow[t]{4}{*}{$10 \times \mathrm{PBS}$} & $92 \mathrm{mM} \mathrm{Na}_{2} \mathrm{HPO}_{4} \cdot 2 \mathrm{H}_{2} \mathrm{O}$ \\
\hline & $16 \mathrm{mM} \mathrm{NaH} 2 \mathrm{PO}_{4} \cdot 2 \mathrm{H}_{2} \mathrm{O}$ \\
\hline & $1.5 \mathrm{M} \mathrm{NaCl}$ \\
\hline & Adjust pH 7.2 with $\mathrm{NaOH}$ \\
\hline \multirow[t]{2}{*}{ 1x PBS- $\mathrm{T}$} & $1 \times \mathrm{PBS}$ \\
\hline & $0.1 \%(\mathrm{v} / \mathrm{v})$ Tween 20 \\
\hline
\end{tabular}




\begin{tabular}{ll}
\hline 2 x SDS-loading buffer & $100 \mathrm{mM}$ Tris-HCl pH 6.8 \\
& $200 \mathrm{mM}$ DTT \\
& $4 \%(w / v)$ SDS \\
& $0.2 \%(w / v)$ Bromophenol blue \\
& $20 \%$ glycerol
\end{tabular}

Stripping membrane buffer

$62.5 \mathrm{mM}$ Tris-HCl pH 6.8

$100 \mathrm{mM} \beta$-mercaptoethanol

$2 \%(w / v)$ SDS

$50 \times \mathrm{TAE}$

2 M Tris-acetate

50 mM EDTA pH 8.0

$10 \times$ TBS

$500 \mathrm{mM}$ Tris- $\mathrm{HCl} \mathrm{pH} 8.0$

$1.37 \mathrm{M} \mathrm{NaCl}$

$27 \mathrm{mM} \mathrm{KCl}$

$1 \times$ TBS-T

$50 \mathrm{mM}$ Tris- $\mathrm{HCl} \mathrm{pH} 8.0$

$137 \mathrm{mM} \mathrm{NaCl}$

$2.7 \mathrm{mM} \mathrm{KCl}$

$0.1 \%(\mathrm{v} / \mathrm{v})$ Tween 20

Tfb I buffer (200 ml)

30 mM K-Acetate

$100 \mathrm{mM} \mathrm{RbCl}$

$10 \mathrm{mM} \mathrm{CaCl}_{2}$

$15 \%(\mathrm{v} / \mathrm{v})$ glycerol

Adjust pH 5.8 with $0.1 \mathrm{M}$ acetic acid

Adjust final volume of $180 \mathrm{ml}$ with deionized $\mathrm{H}_{2} \mathrm{O}$

After autoclaving, $20 \mathrm{ml}$ of sterile-filtered $500 \mathrm{mM}$

$\mathrm{MnCl}_{2} \cdot 2 \mathrm{H}_{2} \mathrm{O}$ was added 
3 Materials and Methods

Tfb II buffer

Western Blot buffer

Yeast alkaline lysis buffer

Buffer A

Buffer B

Buffer C

Buffer D
$10 \mathrm{mM}$ MOPS

$75 \mathrm{mM} \mathrm{CaCl}_{2}$

$10 \mathrm{mM} \mathrm{RbCl}$

$15 \%$ glycerol

Adjust pH 6.5 with $0.5 \mathrm{M} \mathrm{NaOH}$, fresh sterile filtration

$50 \mathrm{mM}$ Tris

$20 \%(\mathrm{v} / \mathrm{v})$ Methanol

$1.441 \%(\mathrm{w} / \mathrm{v})$ glycine

$0.2 \mathrm{M} \mathrm{NaOH}$

$1 \%(w / v)$ SDS

20 mM HEPES-KOH, pH 7.4

$200 \mathrm{mM} \mathrm{KCl}$

$1 \mathrm{mM} \mathrm{MgCl} 2$

20 mM HEPES pH 7.4

$200 \mathrm{mM} \mathrm{KCl}$

$1 \mathrm{mM} \mathrm{MgCl} 2$

$1 \mathrm{M}$ imidazole

25 mM HEPES-KOH pH 7.4

$300 \mathrm{mM} \mathrm{KCl}$

$4 \mathrm{mM} \mathrm{MgCl} 2$

$5 \mathrm{mM} \beta$-mercaptoethanol

25 mM HEPES pH 7.4

$300 \mathrm{mM} \mathrm{KCl}$

$4 \mathrm{mM} \mathrm{MgCl} 2$

$1 \mathrm{M}$ imidazole 


\begin{tabular}{|c|c|}
\hline \multirow[t]{6}{*}{ Buffer E } & 40 mM HEPES-KOH pH 7.4 \\
\hline & $500 \mathrm{mM} \mathrm{KCl}$ \\
\hline & $20 \mathrm{mM} \mathrm{MgCl} 2$ \\
\hline & $5 \%(w / v)$ glycerol \\
\hline & $2 \mathrm{mM} \beta$-mercaptoethanol \\
\hline & $5 \mathrm{mM}$ ATP \\
\hline \multirow[t]{7}{*}{ Buffer F } & 40 mM HEPES pH 7.4 \\
\hline & $500 \mathrm{mM} \mathrm{KCl}$ \\
\hline & $20 \mathrm{mM} \mathrm{MgCl}_{2}$ \\
\hline & $1 \mathrm{M}$ imidazole \\
\hline & $5 \%(\mathrm{w} / \mathrm{v})$ glycerol \\
\hline & $2 \mathrm{mM} \beta$-mercaptoethanol \\
\hline & $5 \mathrm{mM}$ ATP \\
\hline \multirow[t]{2}{*}{ Buffer BA } & $20 \mathrm{mM} \mathrm{KH}_{2} \mathrm{PO}_{4}-\mathrm{KOH} \mathrm{pH} 8.0$ \\
\hline & $5 \mathrm{mM} \beta$-mercaptoethanol \\
\hline \multirow[t]{3}{*}{ Buffer BB } & $20 \mathrm{mM} \mathrm{KH}_{2} \mathrm{PO}_{4}-\mathrm{KOH} \mathrm{pH} 8.0$ \\
\hline & $1 \mathrm{M} \mathrm{NaCl}$ \\
\hline & $5 \mathrm{mM} \beta$-mercaptoethanol \\
\hline \multirow[t]{2}{*}{ Buffer CD } & $10 \mathrm{mM} \mathrm{NaKHPO}_{4}$-buffer $\mathrm{pH} 7.5$ \\
\hline & $0.5 \mathrm{mM} \mathrm{MgCl}_{2}$ \\
\hline \multirow[t]{3}{*}{ Buffer HKM2 } & 10 mM HEPES-KOH pH7.4 \\
\hline & $150 \mathrm{mM} \mathrm{KCl}$ \\
\hline & $1 \mathrm{mM} \mathrm{MgCl} 2$ \\
\hline \multirow[t]{2}{*}{ Buffer GF } & 20 mM HEPES-KOH pH7.4 \\
\hline & $100 \mathrm{mM} \mathrm{KCl}$ \\
\hline
\end{tabular}




\begin{tabular}{|c|c|}
\hline Buffer GFM & 20 mM HEPES-KOH pH7.4 \\
\hline & $100 \mathrm{mM} \mathrm{KCl}$ \\
\hline & $1 \mathrm{mM} \mathrm{MgCl} 2$ \\
\hline Buffer PK & $20 \mathrm{mM}$ Tris- $\mathrm{HCl} \mathrm{pH} 8.0$ \\
\hline & $100 \mathrm{mM} \mathrm{NaCl}$ \\
\hline & $1 \mathrm{mM}$ DTT \\
\hline Buffer QA & 10 mM HEPES-KOH pH7.4 \\
\hline & $1 \mathrm{mM} \mathrm{MgCl}{ }_{2}$ \\
\hline & $10 \mathrm{mM} \mathrm{KCl}$ \\
\hline Buffer QB & 10 mM HEPES-KOH pH7.4 \\
\hline & $1 \mathrm{mM} \mathrm{MgCl} 2$ \\
\hline & $1 \mathrm{M} \mathrm{KCl}$ \\
\hline Buffer SAXS & 20 mM HEPES-KOH pH7.4 \\
\hline & $100 \mathrm{mM} \mathrm{KCl}$ \\
\hline & $1 \mathrm{mM}$ DTT fresh addition \\
\hline
\end{tabular}

Table 6: Buffers

\subsection{Molecular biology methods}

\subsubsection{DNA analytical methods}

DNA concentrations were determined by UV spectroscopy at a wavelength of $260 \mathrm{~nm}$ with a Nanodrop 1000 spectrophotometer (PEQLAB), where one absorption unit corresponds to 50 $\mathrm{ng} / \mu 1$ double stranded DNA. DNA-nuclease free water served as a reference. The absorbance ratio 260/280 nm for pure DNA should be over 1.85. Deviations from this absorbance ration ratio indicate contaminations such as RNA or protein.

DNA electrophoresis was performed with $0.8 \%(\mathrm{w} / \mathrm{v})$ agarose gels submerged in $1 \mathrm{x}$ TAE buffer. To prepare the gels, the agarose was dissolved in TAE buffer containing SYBR-SAFE 
(1:5000). Prior to loading DNA samples on the gel, these were mixed with $6 \mathrm{x}$ DNA loading buffer. Agarose gel electrophoresis was carried out at a constant voltage of $80 \mathrm{~V}$ for 20 minutes.

The authenticity of cloned plasmid constructs was confirmed by restriction digestion with suitable restriction enzymes before DNA sequencing by the Core Facility at the MPI of Biochemistry in Martinsried, Germany. Sequencing chromatograms were visualized by the program Chromas (C. McCarthy, Griffith University, Australia). Comparative sequence analysis was performed by using the MultAlin (http://multalin.toulouse.inra.fr/multalin) analysis online program (Corpet, 1988).

\subsubsection{Polymerase chain reaction (PCR)}

To amplify DNA target sequences from plasmid or annealed PCR oligonucleotides was used. Two kinds of Polymerases, KOD and $P f u$, were used for the amplification of Hip constructs. The following standard protocols and cycling conditions were applied, respectively (Table $7 \&$ Table 8).

\begin{tabular}{ll}
\hline Component & Volume \\
\hline Template $[100 \mathrm{ng}]$ & $0.5 \mu \mathrm{l}$ \\
Primers $[0.5 \mu \mathrm{M}]$ & $1.5 \mu \mathrm{l}$ (each) \\
KOD buffer $10 \mathrm{x}$ & $5 \mu \mathrm{l}$ \\
$\mathrm{MgSO}_{4}$ & $3 \mu \mathrm{l}$ \\
$\mathrm{dNTPs}[2 \mathrm{mM}]$ & $5 \mu \mathrm{l}$ \\
$\mathrm{ddH}_{2} \mathrm{O}$ & $32.5 \mu \mathrm{l}$ \\
$\mathrm{KOD}_{\text {Polymerase }}$ & $1 \mu \mathrm{l}$ \\
Total volume & $50 \mu \mathrm{l}$
\end{tabular}

Table 7: Standard PCR amplification protocol using KOD polymerase. For $P f u$ Polymerase, see Table 9. 


\begin{tabular}{llcc}
\hline & \multicolumn{1}{c}{ Process } & Temperature & Duration \\
\hline Step 1 & Initial denaturation & $95^{\circ} \mathrm{C}$ & $2 \mathrm{~min}$ \\
Step 2 & Cycle denaturation & $94{ }^{\circ} \mathrm{C}$ & $20 \mathrm{~s}$ \\
Step 3 & Primer annealing & $53^{\circ} \mathrm{C}$ & $10 \mathrm{~s}$ \\
Step 4 & Primer extension & $70{ }^{\circ} \mathrm{C}$ & $1 \mathrm{~kb} / \mathrm{min}$ \\
& & & Steps 2-4 (x 35) \\
Step 5 & Final extension & $70{ }^{\circ} \mathrm{C}$ & $5 \mathrm{~min}$ \\
Step 6 & Cooling & $4{ }^{\circ} \mathrm{C}$ & $\infty$ \\
\hline
\end{tabular}

Table 8: Thermocycling conditions for PCR amplification.

\subsubsection{Site-directed mutagenesis}

For site-directed mutagenesis in existing plasmids, a whole-plasmid PCR was performed. A complete list of primer pairs used for site-directed mutagenesis introduced in this study can be found in the Appendix (Table 17). Table 9 and Table 10 list the standard protocol and thermocycling conditions. Modifications to the procedure were made when necessary. The success of the PCR amplification was checked by agarose gel electrophoresis. Afterwards, the product band was excised from the gel, dissolved and the DNA purified. $1 \mu 1 D p n I$ was added to $50 \mu 1$ PCR Clean-Up sample and incubated at $37^{\circ} \mathrm{C}$ for $1 \mathrm{~h}$. The DpnI specifically digest methylated DNA, i.e. the template DNA produced in E.coli. Therefore, the parent DNA strands were selectively removed from the sample (Weiner et al., 1994). Finally, $5 \mu$ l of the sample was directly transformed into E. coli DH5a.

\begin{tabular}{ll}
\hline Component & Volume \\
\hline Template $[100 \mathrm{ng}]$ & $0.5 \mu \mathrm{l}$ \\
Primers $[0.5 \mu \mathrm{M}]$ & $1.5 \mu \mathrm{l}$ (each) \\
Pfu buffer $10 \mathrm{x}$ & $5 \mu \mathrm{l}$ \\
$\mathrm{dNTPs}[2 \mathrm{mM}]$ & $5 \mu \mathrm{l}$ \\
$\mathrm{ddH}_{2} \mathrm{O}$ & $28.5 \mu \mathrm{l}$ \\
Pfu Polymerase & $1 \mu \mathrm{l}$ \\
Total volume & $50 \mu \mathrm{l}$
\end{tabular}

Table 9: Standard PCR amplification protocol using $P f u$ polymerase. 


\begin{tabular}{llcc}
\hline & \multicolumn{1}{c}{ Process } & Temperature & Duration \\
\hline Step 1 & Initial denaturation & $95{ }^{\circ} \mathrm{C}$ & $5 \mathrm{~min}$ \\
Step 2 & Cycle denaturation & $94{ }^{\circ} \mathrm{C}$ & $1 \mathrm{~min}$ \\
Step 3 & Primer annealing & $53{ }^{\circ} \mathrm{C}$ & $1 \mathrm{~min}$ \\
Step 4 & Primer extension & $70{ }^{\circ} \mathrm{C}$ & $1 \mathrm{~kb} / \mathrm{min}$ \\
& & & Steps 2-4 (x 18) \\
Step 5 & Final extension & $70{ }^{\circ} \mathrm{C}$ & $5 \mathrm{~min}$ \\
Step 6 & Cooling & $4{ }^{\circ} \mathrm{C}$ & $\infty$ \\
\hline
\end{tabular}

Table 10: Thermal cycling conditions for site-directed mutagenesis.

\subsubsection{DNA restriction and ligation}

The amplified PCR products or plasmids of interest were digested with the appropriate restriction enzymes at the respective restriction conditions as follows: $19.5 \mu \mathrm{l}$ water, $5 \mu \mathrm{l}$ plasmid $(\sim 0.5 \mu \mathrm{g}), 3 \mu \mathrm{l}$ of the appropriate buffer $\pm 0.5 \mu \mathrm{BSA}, 1 \mu \mathrm{l}$ of each restriction enzyme in $30 \mu \mathrm{l}$ total reaction volume; $50 \mu \mathrm{l}$ PCR product, $5.6 \mu \mathrm{l}$ of appropriate buffer $\pm 0.5 \mu \mathrm{l} \mathrm{BSA}, 1 \mu \mathrm{l}$ of each restriction enzyme. Samples were incubated at $37^{\circ} \mathrm{C}$ for $1 \mathrm{~h}$. The mixtures were separated by agarose gel electrophoresis, and the target bands directly extracted with the PCR Cleanup System (Promega).

For ligation, approximately $50 \mathrm{ng}$ digested plasmid DNA and a three-fold molar excess of insert DNA were mixed with $1 \mu 1$ T4 DNA ligase and $1 \mu \mathrm{T} 4$ ligase buffer in a total volume of $10 \mu$ l. As a control reaction for re-ligation of incompletely restricted plasmid DNA, the same reaction without addition of insert DNA was carried out. The ligations were either performed for $1 \mathrm{~h}$ at room temperature or for $3 \mathrm{~h}$ at $18{ }^{\circ} \mathrm{C}$. Afterwards, $5 \mu 1$ of the ligation product was directly transformed into E. coli DH5 $\alpha$ cells. The cells were subsequently spread on selective agar medium (AMP for pProEx) and incubated overnight at $37^{\circ} \mathrm{C}(\sim 15 \mathrm{~h})$. Successful ligation was confirmed by restriction analysis and subsequent DNA sequencing. 


\subsubsection{Plasmid DNA and DNA fragment purification}

E. coli DH5 $\alpha$ was used for the amplification of plasmid DNA. The transformed strains were grown in LB medium in presence of the appropriate antibiotics as selective markers for 8-16 $\mathrm{h}$ at $37{ }^{\circ} \mathrm{C}$ (Sambrook, 1989). Subsequently, plasmids were isolated by anion exchange chromatography using the Wizard Plus SV Miniprep DNA Purification System, according to the manufacturer's instructions, apart from reducing the washing step to $500 \mu 1$ Membrane Wash Solution in our protocol. For DNA Midiprep and Maxiprep, the Qiagen Plasmid Midi Kit and Qiagen Plasmid Maxi Kit were used, respectively.

To purify DNA fragments after agarose gel electrophoresis or enzymatic reactions (see section 3.6.1 and 3.6.4), the Wizard SV Gel and PCR Clean-Up system were used. Purification and isolation were performed according to the manufacturer's instructions.

\subsubsection{Cloning strategies}

For the present study, a complete list of plasmids constructed and primer pairs used can be found in the Appendix section 8.1, respectively. The expression vectors pProEx-HtA and pProEx-HtB were used for the heterologous expression of protein constructs in E. coli. Proteins were expressed with as an $\mathrm{N}$-terminal $\mathrm{His}_{6}$-tag fusion, which enables easy protein purification by immobilized metal-ion affinity chromatography (IMAC). The N-terminal His ${ }_{6}$-tag was subsequently cleaved with TEV protease.

Cloning of rat Hip constructs: Apart from Hip(1-44) and Hip(312-368), which were cloned into the pETM30 plasmid with restriction sites NcoI and HindIII (Zhuo Li, Master thesis), the Hip constructs were generated by PCR using pProEx-HtB-Hip(1-368) as a template (from Dr. Andreas Bracher), via primers flanked with EheI/HindIII or EheI/KpnI restriction sites. The trimmed amplificates were cloned into the expression plasmid pProEx-HtB (Invitrogen), which was cut with the same respective restriction sites. For generation of Hip mutants, whole plasmid site-directed mutagenesis was used to mutate the residues of interest in the respective plasmid via a PCR based strategy (see section 3.6.3) (Weiner et al., 1994). Primers are listed in Appendix 8.1 (Table 16 and 17). 
Cloning of Hip fusion proteins: To construct Hip(78-234)-linker-Hsp70N and HipM-linkerHsp70N fusion proteins, Hip(78-234) and Hip(78-247) (HipM) were amplified by PCR using primers introducing EheI and EcoRI restriction sites and cloned into plasmid pProEx-Hsp70N (plasmid stock, \#796, Department of Cellular Biochemistry of MPI, Martinsried) between the restriction sites for EheI and EcoRI. The coding sequence for the artificial linker sequences GGS, GGSGGS and GAGGGSGGS between Hip and Hsp70 was constructed by nested PCR method. For construction of the reverse fusion protein Hsp70N-HipM, no artificial linker was introduced, since the $\mathrm{N}$-terminal of Hip encodes an unstructured region, which might work as a flexible tether. XhoI restriction sites were introduced by PCR at the 3' end of Hsp70N and at the beginning of HipM. The EcoRI/XhoI and XhoI/HindIII digested PCR products were subsequently inserted into pProEx-HtA digested with EcoRI and HindIII. For generation of mutants of Hip fusion protein, site-directed mutagenesis with the appropriate primer pairs was employed via PCR (see section 3.6.3) (Weiner et al., 1994). Primers were listed in the Appendix 8.1 (Table 18).

Cloning of Hip constructs into yeast expression plasmids: For cloning the Hip constructs into the yeast expression plasmids p423GPD and p423ADH, SpeI and EcoRI restriction sites were introduced into the Hip constructs by PCR. Before cloning these Hip variants into the yeast plasmids, they were subcloned into a modified pProEx without the original SpeI site. For the Hip mutants, site-directed mutagenesis of this pProEx plasmid with the appropriate primer pairs was performed via PCR (Weiner et al., 1994). Primers are listed in the Appendix 8.1 (Table 19). All plasmid inserts and mutants were verified by DNA sequencing. Subsequently, the inserts were excised from the pProEx-HtB expression plasmid by digestion with SpeI and Eco$R \mathrm{I}$ and cloned into the yeast expression plasmids p423GPD and p423ADH (Mumberg et al., 1995), which were complementarily cleaved with SpeI and EcoRI. Amplification of the constructed yeast expression plasmids was performed in E. coli DH5a.

\subsubsection{Competent $E$. coli cell preparation and transformation}

\subsubsection{Chemocompetent $E$. coli cells and chemical transformation}

For preparation of chemically competent E. coli cells, the $\mathrm{RbCl}$ method was utilized (Hanahan, 1983). A single colony of the respective E. coli strain was grown in $5 \mathrm{ml}$ LB over-night culture. 
$200 \mathrm{ml}$ of fresh Psi broth were inoculated with $2 \mathrm{ml}$ of the overnight culture and grown to an $\mathrm{OD}_{600}$ of 0.5 at $37^{\circ} \mathrm{C}$. Cells were then chilled on ice for $15 \mathrm{~min}$ and mixed frequently to speed up the cooling process. Afterwards, the cell suspensions were transferred into $50 \mathrm{ml}$-Falcon and centrifuged at $4{ }^{\circ} \mathrm{C}$ for $20 \mathrm{~min}$ at $3700 \mathrm{rpm}$. The supernatant was removed, and the cell pellets resuspended by gentle shaking in $40 \mathrm{ml}$ freshly prepared, ice-chilled TfbI buffer. After incubation on ice for $15 \mathrm{~min}$, the mixture was centrifuged again for $10 \mathrm{~min}$ at $3700 \mathrm{rpm}$ at $4{ }^{\circ} \mathrm{C}$. Following decantation of the supernatant, the harvested cells were placed on ice and resuspended in $8 \mathrm{ml}$ ice-chilled TfbII buffer by gentle shaking during $15 \mathrm{~min}$ on ice. Subsequently, $50 \mu \mathrm{l}$ aliquots of the cell suspension were pipetted into pre-cooled tubes $\left(-20^{\circ} \mathrm{C}\right)$, and immediately frozen in liquid nitrogen. Afterwards, the tubes were stored at $-80^{\circ} \mathrm{C}$.

For transformation, $50 \mu \mathrm{l}$ chemically competent $E$. coli cells were mixed with $50-100 \mathrm{ng}$ plasmid DNA or $5 \mu \mathrm{l}$ ligation mixture, and incubated on ice for $30 \mathrm{~min}$. Subsequently, the cells were heat-shocked at $37^{\circ} \mathrm{C}$ for $5 \mathrm{~min}$ and afterwards immediately cooled on ice for $1 \mathrm{~min} .950$ $\mu \mathrm{l}$ of LB were added to the cells and incubated for $1 \mathrm{~h}$ at $37^{\circ} \mathrm{C}$ under gentle shaking (450 rpm). The cell suspension was then plated on plates with selective agar LB medium and incubated overnight at $37{ }^{\circ} \mathrm{C}(\sim 15 \mathrm{~h})$. Single colonies of transformants were picked and inoculated in liquid medium for further analysis.

\subsubsection{Electrocompetent $E$. coli cells and electroporation}

Electrocompetent $E$. coli cells were made by inoculating $500 \mathrm{ml}$ of LB medium with $1 \mathrm{ml}$ overnight culture and grown at $37^{\circ} \mathrm{C}$ to $\mathrm{OD}_{600}$ of 0.5 . Subsequently, cells were chilled on ice for $15 \mathrm{~min}$ and centrifuged for $15 \mathrm{~min}$ at $4200 \mathrm{rpm}$ (Beckmann centrifuge Avanti J-25 with rotor JS 4.2) at $4{ }^{\circ} \mathrm{C}$. Followed supernatant decantation, cell pellets were resuspended in $100 \mathrm{ml}$ cold sterile water followed by centrifugation as described above. The washing step was repeated and cells were resuspended in $50 \mathrm{ml}$ pre-cooled sterile $10 \%(\mathrm{v} / \mathrm{v})$ glycerol. Centrifugation was carried out for $10 \mathrm{~min}$ at $3700 \mathrm{rpm}$ (Beckmann centrifuge Avanti J-25 with rotor JA 25.50) at $4{ }^{\circ} \mathrm{C}$ and the cell pellet was resuspended in $1 \mathrm{ml}$ of pre-cooled sterile $10 \%(\mathrm{v} / \mathrm{v})$ glycerol. Each $50 \mu \mathrm{l}$ were aliquoted into pre-cooled tubes $\left(-20^{\circ} \mathrm{C}\right)$, flash-frozen in liquid nitrogen and stored at $-80{ }^{\circ} \mathrm{C}$. 
Before the start of the electroporation, electroporation cuvettes were pre-cooled and the competent cells were thawed on ice. $5 \mu \mathrm{l}$ of the ligation mix or $\sim 100 \mathrm{ng}$ of plasmid DNA were transferred to the cells, and this mixture was subsequently transferred into the electroporation cuvette and pulsed (1250 V, $25 \mu \mathrm{F}, 200 \Omega) .800 \mu \mathrm{LB}$-medium were immediately added into the cuvette after the signal tone. The mixture was subsequently transferred into an Eppendorf tube and incubated for $1 \mathrm{~h}$ at $37{ }^{\circ} \mathrm{C}$ with $450 \mathrm{rpm}$. Afterwards, the cells were pelleted for $1 \mathrm{~min}$ at $7000 \mathrm{rpm}$ (Eppendorf 5415D). Cells were resuspended in $\sim 100 \mu \mathrm{l}$ medium, plated on selective LB agar medium and incubated overnight at $37^{\circ} \mathrm{C}(\sim 15 \mathrm{~h})$.

\subsubsection{Lithium acetate transformation of $S$, cerevisiae cells}

All yeast transformations in this study were performed following to the lithium acetate (LiAc) method (Gietz et al., 1995; Schiestl and Gietz, 1989). A single colony of the S. cerevisiae strain DSY-1100 (Genotype MATa leu2-112, ura3-1, trp1-1, his3-11, 15 ade2-1, can1-100 GAL SUC2 carrying plasmids pDS-125 (GR/TRP) and pDS-362 (GRE-lacZ/URA)) was picked from a freshly streaked SC-Ade-Leu plate and inoculated into $5 \mathrm{ml} \mathrm{SC-Ade-Leu} \mathrm{medium} \mathrm{as} \mathrm{a} \mathrm{start}$ overnight culture, which was incubated at $30{ }^{\circ} \mathrm{C}$ with vigorous shaking $200 \mathrm{rpm}$. This culture was diluted with SC-Ade-Leu medium in a $200 \mathrm{ml}$ flask to an $\mathrm{OD}_{600}$ of $\sim 0.15$ in a total volume of $50 \mathrm{ml}$. Cells were harvested at $\mathrm{OD}_{600}$ of 0.5 by centrifugation for $5 \mathrm{~min}$ with a speed of 3700 rpm at $4{ }^{\circ} \mathrm{C}$. The yeast pellet was suspended in $25 \mathrm{ml}$ autoclaved water and centrifuged again. The resulting cell pellet was washed with $1 \mathrm{ml} 100 \mathrm{mM}$ LiAc and transferred into a $1.5 \mathrm{ml}$ Eppendorf tube, which was centrifuged for $15 \mathrm{~s}$ with top speed. The cell pellet was resuspended in $500 \mu \mathrm{l} 100 \mathrm{mM} \mathrm{LiAc}$ and chilled on ice. For each transformation, $50 \mu \mathrm{l}$ cells were needed. Cells were centrifuged at $2000 \mathrm{rpm}$ for 1 minute. After removal of the LiAc solution, the following components were added sequentially: $240 \mu 1$ sterile-filtered 50 \% PEG3350, $36 \mu 11 \mathrm{M}$ LiAc, $5 \mu 110 \mathrm{mg} / \mathrm{ml}$ herring testes carrier DNA, $60 \mu \mathrm{l}$ autoclaved water and $5 \mu \mathrm{l}$ plasmid DNA (p423GPD-Hip or p423ADH-Hip) at a concentration of $100 \mathrm{ng} / \mu \mathrm{l}$. Each tube was vigorously vortexed at room temperature until all the components were mixed well, then incubated in a shaker at $600 \mathrm{rpm}$ and $30{ }^{\circ} \mathrm{C}$ for $30 \mathrm{~min}$. Cells were then heat-shocked at $42{ }^{\circ} \mathrm{C}$ for $20 \mathrm{~min}$ and centrifuged at $8000 \mathrm{rpm}$ for $30 \mathrm{~s}$. Afterwards, the cell pellet was resuspended in $1 \mathrm{ml} \mathrm{SC}$ medium and incubated at $30{ }^{\circ} \mathrm{C}$ and $600 \mathrm{rpm}$. After $2 \mathrm{~h}$, the cells were harvested by centrifugation at 
$8000 \mathrm{rpm}$ for $1 \mathrm{~min}$ at room temperature and resuspended in $100 \mu \mathrm{l}$ of appropriate selective medium for plating on SC-Ade-Leu-His medium. These plates were then incubated for maximal two days at $30^{\circ} \mathrm{C}$.

\subsection{Protein biochemical and biophysical methods}

\subsubsection{Protein expression and purification}

For protein overexpression in a BL21 codon plus stain, 1 L of LB medium containing suitable antibiotics was directly inoculated with $\sim 1 \mathrm{ml}$ of the transformation mixture grown overnight at $33{ }^{\circ} \mathrm{C}$ with $180 \mathrm{rpm}$ of gentle shaking until next morning $(\sim 15 \mathrm{~h})$, following incubation, the cells were further grown at $37^{\circ} \mathrm{C}$ with $200 \mathrm{rpm}$ to $\mathrm{OD}_{600}$ of 0.7 .

Protein purifications were performed at $4{ }^{\circ} \mathrm{C}$ unless stated. All the steps of purification were monitored by SDS-PAGE, and protein concentrations were measured by Bradford assay and $\mathrm{A}_{280}$.

\subsubsection{Hip constructs}

Firstly, constructs Hip(1-368), Hip(1-267), Hip(78-267) and Hip(78-368) were cloned by Dr. Andreas Bracher. Genes encoding Hip(1-44) and Hip(312-368) were cloned into bacterial expression vector pETM30 (Zhuo Li, Master thesis) and the other constructs Hip(45-267), Hip(45-368), Hip(78-234), Hip(78-247) and Hip(107-267) were cloned into pProEx-HtB containing an N-terminal 6x His tag. The proteins were expressed in the E.coli strain BL21 (DE3) codon Plus RIL (Novagen) at $37{ }^{\circ} \mathrm{C}$ in LB medium containing $0.1 \mathrm{mg} / \mathrm{ml}$ AMP (ampicillin for pProEx) or $0.025 \mathrm{mg} / \mathrm{ml} \mathrm{Kan} \mathrm{(kanamycin} \mathrm{for} \mathrm{pETM30)} \mathrm{and} 0.034 \mathrm{mg} / \mathrm{ml} \mathrm{CM}$ (chloramphenicol). The cultures were grown at $37{ }^{\circ} \mathrm{C}$ until $\mathrm{OD}_{600}$ of 0.7 and expression was induced with 0.5 $\mathrm{mM}$ IPTG at $37{ }^{\circ} \mathrm{C}$ for $3 \mathrm{~h}(\mathrm{Hip}(1-44)$ and $\mathrm{Hip}(312-368))$ or at $18{ }^{\circ} \mathrm{C}$ for $16 \mathrm{~h}$ (all other constructs). The harvested cells were removed by centrifugation at $4000 \mathrm{rpm}$ (Beckman Coulter J6Micentrifuge with JS 4.2 rotor) and $4{ }^{\circ} \mathrm{C}$ for $25 \mathrm{~min}$. Each $1 \mathrm{~L}$ cell pellet was resuspended in 20 $\mathrm{ml}$ cell lysis buffer A and supplemented with $1 \mathrm{mM}$ PMSF. Subsequently the cells were mechanically disrupted by ultrasonication with a Misonix Sonicator 3000 cell disruptor. The cells were placed in an ice bath and treated with 12 cycles of $25 \mathrm{~s}$ pulsing with force 6.0 and $95 \mathrm{~s}$ 
chilling. Cell debris was clarified by centrifugation at a speed $20000 \mathrm{rpm}$ (Beckmann centrifuge Avanti J-25 with JA 25.50 rotor) and $4{ }^{\circ} \mathrm{C}$ for $40 \mathrm{~min}$. Supernatant was loaded onto a $\mathrm{Ni}$ -

Chelating Sepharose (GE Healthcare), which was equilibrated with $1 \%$ buffer B (dilution with buffer A) (Table 6). The bound protein was eluted with an imidazole gradient of increasing buffer B content, and fractions containing protein were pooled. For cleavage of the eluted Histagged protein, the protein was incubated with TEV protease at a mass ratio of 1:100 in the presence of $1 \mathrm{mM}$ DTT and $0.5 \mathrm{mM}$ EDTA at $4{ }^{\circ} \mathrm{C}$ overnight. The uncleaved His-tagged protein and TEV protease were removed on Ni-Chelating Sepharose (GE Healthcare). Before loading on Ni-Cleating Sepharose, the imidazole-containing buffer was removed by buffer exchange against buffer HKM2 (Table 6) using a HiPrep 26/10 Desalting column (GE Healthcare). If necessary, TEV-cleaved protein in flow-through fractions was further purified by additional anion-exchange chromatography (MonoQ, GE Healthcare) using a linear gradient of 0.01 to $1 \mathrm{M} \mathrm{KCl}$ mixed from buffers QA and QB (Table 6). Finally the proteins were purified by size exclusion chromatography on the Superdex 200 (GE Healthcare) using the buffer GF (Table 6) as equilibration and running buffer. The purity and molecular mass were verified by SDS-PAGE.

\subsubsection{Hip fusion constructs}

Hip-Hsp70 fusion proteins were expressed and purified similar to Hip variants. The modifications are described in the following. Overnight expression $(\sim 16 \mathrm{~h})$ of the fusion proteins at $18{ }^{\circ} \mathrm{C}$ was induced with $0.8 \mathrm{mM}$ IPTG. Harvested cell pellets from $2 \mathrm{~L}$ were resuspended in 40 $\mathrm{ml} 1 \%$ buffer D (diluted with buffer C) (Table 6), supplemented with $1 \mathrm{mM}$ PMSF and $1 \mathrm{x}$ Complete protease inhibitor cocktail. The His $_{6}$-tagged fusion protein was eluted using an imidazole gradient of increasing buffer D. In the size exclusion chromatography on the Superdex 200 (GE Healthcare), the running buffer was GF (Table 6) containing 1 mM DTT. The protein was directly pooled and concentrated to $16-20 \mathrm{mg} / \mathrm{ml}$. Aliquots of this material were flashfrozen in liquid nitrogen and stored at $-80^{\circ} \mathrm{C}$. 


\subsubsection{Hsp70 homologs}

All the full-length human Hsp70 constructs (wild type, Hsp70(D199A) and Hsp70(T204A))

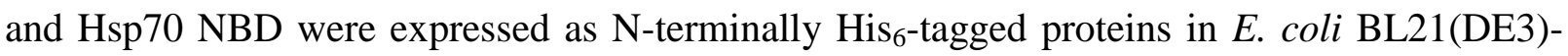
Codon Plus RIL cells. All the strains were grown at $37^{\circ} \mathrm{C}$ in LB medium containing $0.1 \mathrm{mg} / \mathrm{ml}$ ampicillin and $0.034 \mathrm{mg} / \mathrm{ml}$ chloramphenicol. At an $\mathrm{OD}_{600}$ of 0.7 , full-length proteins and Hsp70 NBD were induced with $0.2 \mathrm{mM}$ IPTG and $0.8 \mathrm{mM}$ IPTG, respectively. Cells were subsequently grown at $18{ }^{\circ} \mathrm{C}$ for $16 \mathrm{~h}$. Cells were harvested by centrifugation at $4{ }^{\circ} \mathrm{C}$ and $4200 \mathrm{rpm}$ for $25 \mathrm{~min}$. The pellets were suspended in $1 \%$ buffer $\mathrm{F}$ (diluted with buffer E) for full-length Hsp70 variants or $1 \%$ buffer D (diluted with buffer C) for Hsp70 NBD containing $1 \mathrm{mM}$ PMSF and Complete protease inhibitor cocktail and lysed by ultrasonication as described above in 3.7.1.1. After 30 minutes centrifugation at $4{ }^{\circ} \mathrm{C}$ with $20000 \mathrm{rpm}$, the supernatant will be loaded onto Ni-HiTrap Chelating Sepharose (GE Healthcare), which was pre-equilibrated with $50 \mathrm{ml} 1 \%$ buffer F (full-length Hsp70) or $1 \%$ buffer D (Hsp70 NBD) containing 10 mM imidazole. The bound protein was eluted with a linear gradient of imidazole. The fractions containing highly concentrated protein were pooled together. For cleavage of $\mathrm{His}_{6}$-tag, the pool of protein was incubated with $\mathrm{His}_{6}$-TEV protease at a molar ratio of 1:100 in the presence of $1 \mathrm{mM}$ DTT and $0.5 \mathrm{mM}$ EDTA at $4{ }^{\circ} \mathrm{C}$ overnight. To remove the residual cleaved His ${ }_{6}$-tagged protein and TEV protease, the proteins were first transferred into HKM2 buffer (Table 6) and then passed onto Ni-HiTrap Chelating Sepharose again, which was equilibrated with $2 \%$ buffer F (full-length Hsp70) or $2 \%$ buffer D (Hsp70 NBD) containing $20 \mathrm{mM}$ imidazole. The flowthrough containing the protein without $\mathrm{His}_{6}$-tag was pooled. After the Ni-affinity chromatography, anion exchange MonoQ column chromatography was performed. Before loading onto MonoQ (GE Healthcare), proteins were changed into buffer QA containing low salt by using HiPrep 26/10 Desalting column. When necessary, size exclusion chromatography on Superdex 200 using buffer GF (Table 6) followed as the final purification step. Purified proteins were flash-frozen in liquid nitrogen and stored at $-80{ }^{\circ} \mathrm{C}$.

To remove the bound nucleotides from the full-length Hsp70, after $2^{\text {nd }}$ Ni-affinity chromatography, the protein was incubated with $50 \mathrm{mM}$ EDTA on a rotation plate at room temperature for $1 \mathrm{~h}$. Subsequently the nucleotide was partially removed by the size exclusion chromatography on Superdex 200 (GE Healthcare) with running buffer GF. After Superdex 200, Hsp70 was incubated with Calf Intestinal Alkaline Phosphatase (CIP, New England Biolabs) at a ratio 
of $3 \mathrm{U} / \mathrm{mg} \mathrm{Hsp} 70$ at room temperature for $3 \mathrm{~h}$ and then incubated on ice overnight. CIP phosphatase and reaction products were removed by size exclusion chromatography on Superdex 200.

Another effective method to remove nucleotide of Hsp70 NBD is using the HiTrap Blue Sepharose column (GE Healthcare), which was described in a previous study (Arakawa et al., 2011). After Hsp70N was purified with anion exchange chromatography, protein buffer was exchanged to buffer BA (Table 6) using HiPrep 26/10 Desalting column (GE Healthcare). Protein was loaded onto a $5 \mathrm{ml}$ the HiTrap Blue Sepharose which was equilibrated with buffer BA, and eluted with buffer $\mathrm{BB}$ containing $1 \mathrm{M} \mathrm{NaCl}$ salt gradient in $0.5 \mathrm{ml} / \mathrm{min}$ flow-rate (Table 6).

\subsubsection{Protein analytical methods}

\subsubsection{Protein quantification}

Protein concentrations were determined either colorimetrically using the Bradford assay or spectrophotometrically by measuring the absorption at $280 \mathrm{~nm}$. Samples of cell lysate or protein mixtures were quantified by the Bradford assay according to the manufacturer's instructions (Bradford 1976). 1-5 $\mu \mathrm{l}$ protein were added to $1 \mathrm{ml}$ of a 1:4 dilution Bio-Rad protein assay reagent and the absorbance at $595 \mathrm{~nm}$ measured with a DU640 photometer (Beckman Coulter) against the reagent background. Concentrations of pure proteins were always determined by $\mathrm{A}_{280}$ using a Nanodrop 1000 (PEQLAB). As a reference, protein-free buffer was used. Concentration was determined with the theoretical molar extinction coefficient $\left(\mathrm{M}^{-1} \mathrm{~cm}^{-1}\right)$, which was calculated based on the protein sequence with the online program ExPASy ProtParam (http://web.expasy.org/protparam).

\subsubsection{SDS-PAGE}

To separate and analyze protein mixtures based on molecular weight, sodium dodecyl sulfate polyacrylamide gel electrophoresis (SDS-PAGE) was used (Laemmli, 1970). Protein analysis was performed under denaturing and reducing conditions. Gels were prepared as described in Table 11 and water was layered over the resolving gel while the gel matrix polymerized. Protein samples were mixed with 2 x SDS-sample buffer. Prior to loading, samples were heated for 
5 min at $95{ }^{\circ} \mathrm{C}$ and spun down shortly at maximum speed in a table centrifuge (Eppendorf 5415D). Gel electrophoresis was completed in Bio-Rad Mini-Protean 2 electrophoresis chambers filled with SDS-PAGE running buffer employing a constant voltage of $200 \mathrm{~V}$ for $45 \mathrm{~min}$.

\begin{tabular}{cccc|c}
\hline \multirow{2}{*}{$\begin{array}{c}\text { Solutions } \\
\text { (for 4 gels) }\end{array}$} & \multicolumn{3}{c|}{ Resolving gel } & Stacking gel \\
\cline { 2 - 5 } deionized water & $7.9 \mathrm{ml}$ & $6.6 \mathrm{ml}$ & $4.6 \mathrm{ml}$ & $2.7 \mathrm{ml}$ \\
$1.5 \mathrm{M}$ Tris-HCl pH 8.8 & $5 \mathrm{ml}$ & $5 \mathrm{ml}$ & $5 \mathrm{ml}$ & - \\
$1.0 \mathrm{M}$ Tris-HCl pH 6.8 & - & - & - & $0.5 \mathrm{ml}$ \\
$30 \%$ Acrylamide & $6.7 \mathrm{ml}$ & $8.0 \mathrm{ml}$ & $10.0 \mathrm{ml}$ & $0.67 \mathrm{ml}$ \\
$10 \%$ (w/v) SDS & $200 \mu \mathrm{l}$ & $200 \mu \mathrm{l}$ & $200 \mu \mathrm{l}$ & $40 \mu \mathrm{l}$ \\
$10 \%$ (w/v) APS & $200 \mu \mathrm{l}$ & $200 \mu \mathrm{l}$ & $200 \mu \mathrm{l}$ & $40 \mu \mathrm{l}$ \\
TEMED & $8 \mu \mathrm{l}$ & $8 \mu \mathrm{l}$ & $8 \mu \mathrm{l}$ & $4 \mu \mathrm{l}$ \\
\hline
\end{tabular}

Table 11: Composition of resolving and stacking gels for SDS-PAGE.

\subsubsection{Coomassie blue staining of SDS-PAGE}

Coomassie blue staining was used for the visualization of protein bands in SDS-PAGE. Coomassie blue dye is a triphenylmethane coloring agent that binds to proteins. The dye integrates with cationic, nonpolar and hydrophobic side chains of a polypeptide. Protein bands were fixed and stained in the gel matrix by immersion into freshly prepared Coomassie blue staining solution, followed by gentle shaking for 20 min. The background destaining was performed by incubation with Coomassie destaining solution for at least $1 \mathrm{~h}$ at RT under gentle shaking.

\subsubsection{Western blotting and immunodetection}

Proteins were separated on SDS-PAGE and subsequently proteins were transferred from the gel onto nitrocellulose membranes using a semi-dry western blot system (SemiPhor). Transfer was performed in Western blot buffer and accomplished by employing a constant current of $80 \mathrm{~mA}$ for $75 \mathrm{~min}$ for one or two gels. Transfer efficiency was determined by staining with Ponceau $\mathrm{S}$ solution for 2-3 min, followed by water destaining to remove background. Membranes were afterwards immediately subjected to immunodetection. 
Nitrocellulose membranes were blocked in 3-5\% milk powder in PBST/TBST solution for $1 \mathrm{~h}$ at RT. The primary antibody was diluted in blocking solution and incubated with the membrane for $2 \mathrm{~h}$ at room temperature or at $4{ }^{\circ} \mathrm{C}$ overnight. The membranes were washed for 3-4 times with TBST solution, each time 10 min long. After washing, the membrane was incubated with HRP-conjugated (horseradish peroxidase) secondary antibody at a dilution of 1:5000 in TBST for $1 \mathrm{~h}$ at RT. The membranes were washed again as above and the bound HRP-coupled antibodies were detected by enhanced chemiluminescence (ECL) staining using a 1:1 ratio of Rodeo ECL detection reagents 1 and 2. The detection reagent should completely cover the membranes. Chemiluminescence was detected and documented with the Fuji-LAS3000 luminescence and densitometry system. When necessary, membranes were stripped with stripping buffer at $70{ }^{\circ} \mathrm{C}$ for $45 \mathrm{~min}$.

\subsubsection{Circular dichroism spectroscopy (CD)}

The secondary structure of purified Hip variants was determined by CD spectroscopy. Proteins were exchanged into buffer CD by using Superdex 200 (Table 6). The CD spectra were measured with a Jasco-715 spectrometer equipped with a Peltier-thermostat. $200 \mu 1$ of each protein sample with concentration of $\sim 0.1 \mathrm{mg} / \mathrm{ml}$ or $2.5 \mu \mathrm{M}$ was analyzed in $0.1 \mathrm{~cm}$ glass cuvettes at $4{ }^{\circ} \mathrm{C}$. Wavelength scans ranging from 250-195 $\mathrm{nm}$ were recorded four times and averaged, followed by correction with the analysis buffer baseline. All the spectra were fitted with the programs CDPro analysis and CONTIN to estimate the secondary structure content.

\subsubsection{Small angle $X$-ray scattering (SAXS)}

Samples of Hip constructs for SAXS measurement were applied to an analytical Superdex 200 column operated at $18{ }^{\circ} \mathrm{C}$ in buffer SAXS (see Table 6). Three different concentrations: $\sim 2,5$ and $10 \mathrm{mg} / \mathrm{ml}$ were prepared. After concentrations were accurately measured with the Nanodrop photometer ( $2 \mu$ l loading), $1 \mathrm{mM}$ fresh DTT was added to each sample. The SAXS experiments were performed with a monochromatic X-ray beam of $0.931 \AA$ wavelength at ESRF beamline ID14-3. As a reference, a BSA sample was measured. For each Hip sample, ten measurements of $10 \mathrm{~s}$ exposures were recorded and averaged. The buffer background scattering curve was subtracted. The program Primus was used to process the protein scattering data 
(Konarev et al., 2003). Radii of gyration $\left(\mathrm{R}_{\mathrm{g}}\right)$ were determined using the Guinier approximation. Scattering curves were fitted with GNOM (Svergun, 1992).

\subsubsection{Multi-angle light scattering (MALS)}

For determination of molecular mass and hydrodynamic radium of Hip fusion proteins, size exclusion chromatography/multi-angle light scattering (MALS) was performed by Dr. Manajit Hayer-Hartl. $80 \mu \mathrm{l}$ of each Hsp70N-HipM and HipM-Hsp70N were respectively injected on a gel filtration column using a $100 \mu \mathrm{l}$ injection loop. The column was developed at a flow rate $0.2 \mathrm{ml} / \mathrm{min}$. The GFM buffer used as running buffer contained $1 \mathrm{mM}$ fresh DTT. The system was connected to DAWN EOS MALS detector (960 nm laser), variable wavelength detector (absorbance at $280 \mathrm{~nm}$, Agilent 1100 series), and Optilab DSP refractive index detector (690 $\mathrm{nm})$. Masses and molecular diameters were calculated using the ASTRA software with the $\mathrm{dn} / \mathrm{dc}$ value set to $0.185 \mathrm{mg} / \mathrm{g}$.

\subsubsection{Protein crystallization and structure determination}

\subsubsection{Crystallization}

Before setting up the crystallization screens, particles and precipitated protein were first removed from the protein solution by centrifugation with $12000 \mathrm{rpm}$ at $4{ }^{\circ} \mathrm{C}$ for $40 \mathrm{~min}$. Initial crystal screens were conducted at the Crystallization Facility of the MPI of Biochemistry, Martinsried. In the initial screening, the sitting drop vapor diffusion method was used mixing 100 $\mathrm{nl}$ protein solution and $100 \mathrm{nl}$ reservoir solution.

The precipitant kits Hampton Research Index, Qiagen Classics, Qiagen pH Clear 1 and Qiagen $\mathrm{pH}$ Clear 2 were used for initial screenings at $18{ }^{\circ} \mathrm{C}$ for Hip(78-247), named HipM in the following. Initial crystals of HipM were obtained from the Hampton Research Index and Qiagen $\mathrm{pH}$ Clear 1 screens with 1.4 M tri-sodium citrate, 0.1 M HEPES pH 7.5 and 2.4 M Na-malonate $\mathrm{pH}$ 7.0. Crystallization conditions were optimized by systematically varying the precipitant concentration. To get larger crystals, $1 \mu \mathrm{l} \mathrm{HipM}$ at the same concentration, $18 \mathrm{mg} / \mathrm{ml}$, was mixed with $1 \mu \mathrm{l}$ of reservoir solution and equilibrated against $500 \mu \mathrm{l}$ of reservoir solution at $18{ }^{\circ} \mathrm{C}$ using the hanging drop vapor diffusion method. Crystal grew in clusters of thin plates. 
Optimization of the tri-sodium citrate condition was not successful. With the Na-malonate $\mathrm{pH}$ 7.0 condition, single crystals can hardly be achieved. Thin plates were only observed after several rounds of micro-seeding. For this crystal form, experimental phasing using heavy atom soaks failed. Growing similar crystals of SeMet-substituted HipM also failed. This protein formed spheroids, and single crystal could not be obtained by seeding. However, a second crystal form was found at $\mathrm{pH} 6.0$ with sodium malonate as precipitant. Single, cube-shaped tetragonal crystals were obtained after three weeks in presence of $2.3 \mathrm{M} \mathrm{Na}$-malonate $\mathrm{pH} 6.0$ at $18{ }^{\circ} \mathrm{C}$. For cryo-protection of the crystals, they were transferred in two steps into a cryo-solution. First they were mixed with a 1:1 mixture of mother liquor and cryo-solution for $15 \mathrm{~min}$. The mother liquor contained 2.5 M Na-malonate $\mathrm{pH} 6.0$ and the cryo-solution with additionally $15 \%$ glycerol. After equilibration, they were transferred to $100 \%$ cryo-solution and incubated for 15 min, followed by flash-cooling in liquid nitrogen.

To crystallize the Hsp70N-HipM fusion protein, the precipitant kits Qiagen PEGs, Hampton Research Index and Qiagen Classics Lite were used for initial screening at $4{ }^{\circ} \mathrm{C}$ and $18{ }^{\circ} \mathrm{C}$. Initial crystals were obtained from Qiagen PEGs screening kit with $0.2 \mathrm{M} \mathrm{NH}_{4} \mathrm{I}$ and $20 \%$ PEG 3350 at $4{ }^{\circ} \mathrm{C}$ and $18{ }^{\circ} \mathrm{C}$. Improved crystals were achieved by hanging drop vapor diffusion method after micro-seeding in presence of $0.2 \mathrm{M} \mathrm{NH}_{4} \mathrm{I}$ and $17 \%$ PEG 3350 at $18{ }^{\circ} \mathrm{C}$. For cryoprotection, the original drop was first mixed with a 1:1 mixture of mother liquor and cryosolution for $15 \mathrm{~min}$. The mother liquor was $0.2 \mathrm{M} \mathrm{NH}_{4} \mathrm{I}, 20 \% \mathrm{PEG} 3350$ and cryo-solution with additional $15 \%$ glycerol. Finally, they were transferred to $100 \%$ cryo-solution and incubated for $15 \mathrm{~min}$, followed by flash-cooling in liquid nitrogen.

\subsubsection{Data collection, structure solution and refinement}

Diffraction data were collected at beamlines ID14-4, ID23-1, ID23-2 and ID29 of ESRF Grenoble, France. The data collection strategy was devised with iMosflm from two wedges of reflection images $90^{\circ}$ apart (Battye et al., 2011). The images were integrated with XDS (Kabsch, 2010) and converted to CCP4 format with Pointless (Evans, 2006). SCALA (Evans, 1997) was utilized for scaling as implemented in the CCP4i interface (Collaborative Computational Project, 1994). 
HipM crystal form I ( $\left.\mathbf{P 4}_{3} \mathbf{2}_{1} \mathbf{2}\right)$ : The native dataset was from a crystal soaked with hydroxylmercuribenzoic acid. The mercury ligand was however not bound or disordered in the structure. The form I structure of HipM was initially determined by single isomorphous replacement with anomalous scattering (SIRAS) from this native dataset and derivative dataset from a $\mathrm{K}_{2}\left[\mathrm{OsO}_{4}\right]$ soaked crystal by direct methods using Shelx-CDE as implemented in HKL2MAP at 5.6 resolution (Schneider and Sheldrick, 2002). SHARP was used to refine the three heavy atom sites and for calculating initial phases (Vonrhein et al., 2007). Phase extension and density modification was performed with Resolve, resulting in an interpretable electron density map (Terwilliger, 2000). The unit cell contained two copies of HipM, which were symmetrically associated via C-terminal interaction with the opposing neighbor molecule. Density for the residues 78-93 and 213-247 was not discernible. The respective portions of the protein were either disordered or cleaved off in both copies. The final model was refined against native dataset of $2.6 \AA$ resolution.

HipM crystal form II (P2 $)$ : The diffraction data was initially integrated in the orthorhombic space group C222 ${ }_{1}$ with lattice parameters of 68.9, 92.4 and $126.6 \AA$. This structure was solved by molecular replacement with the HipM fragment coordinates from the Hsp70N-HipM fusion protein using Molrep (Vagin and Isupov, 2001). However, refinement of the model failed to lower the R-factors. The actual space group turned out to be $\mathrm{P} 22_{1}$ with quite similar lattice parameters a and c with $57.29 \AA$ and $57.31 \AA$, respectively. The crystals were twinned and each unit cell contained four copies of HipM. Residues 82-241 were ordered in all chains.

Hsp70N-HipM fusion protein: This fusion protein was solved by molecular replacement using Molrep with the Hsp70(1-393)-ADP complex and the HipM monomer from crystal form I as search templates (Vagin and Isupov, 2001). Residues 1-382 of human Hsp70 and residues $78-243$ of rat Hip were defined in the density as well as $\mathrm{ADP}-\mathrm{Mg}^{2+}$ and 17 iodine ions. The positions of 17 resolved iodine ions from the precipitant solution were confirmed from the anomalous signal in the dataset collected from another crystal at $2.0 \AA$ A wavelength.

All of the models were built interactively using Coot (Emsley and Cowtan, 2004). Refinement was performed with REFMAC5 (Murshudov et al., 1997). Non-glycine residues without detectable side chain density facing solvent channels were modeled as alanines. Together with the 
previous solved dimerization domain of Hip, all of the atomic coordinates and structure factors of HipM and Hsp70N-HipM fusion protein have been deposited in the Protein Data Bank under the accession code of 4J8C (HipN), 4J8E (HipM, crystal form I), 4J8D (HipM, crystal form II) and 4J8F (Hsp70N-HipM). Structural figures were generated with PyMOL (Schrödinger, 2010).

\subsubsection{Structure analysis}

Coordinates were aligned with Lsqman (Kleywegt and Jones, 1994). The sequence alignment was prepared with ClustalW (Larkin et al., 2007; Thompson et al., 1994) and ESPript (Gouet et al., 1999). The interaction surface in the Hip:Hsp70 complex was analyzed with Areaimol (Lee and Richards, 1971), Contact (Skarzynski, 1988), Naccess (Hubbard and Thornton, 1993). Surface shape complementarity between Hip and Hsp70 complex was estimated using SC (Lawrence and Colman, 1993).

\subsubsection{Functional characterization in vitro}

\subsubsection{Limited proteolysis with Proteinase $\mathrm{K}$}

In order to determine stably folded protein fragments, protein samples were subjected to Proteinase $\mathrm{K}$ treatment. Aliquots of $6 \mu \mathrm{M}$ purified protein were incubated with a series of Proteinase $\mathrm{K}$ concentrations from 0.4 to $2.56 \mu \mathrm{g} / \mathrm{ml}$ on ice for $1 \mathrm{~h}$. The reaction was then quenched by addition of $100 \mathrm{mM}$ PMSF to a final concentration of $4 \mathrm{mM}$ PMSF, followed by 5 minutes incubation on ice. To remove insoluble PMSF, the samples were centrifuged for $1 \mathrm{~min}$. $10 \mu \mathrm{l}$ supernatant were mixed with $10 \mu 12$ x SDS loading buffer and heated to $95{ }^{\circ} \mathrm{C}$ for $5 \mathrm{~min}$. The samples were analyzed by SDS-PAGE on $15 \%$ acrylamide gels after Coomassie blue staining.

\subsubsection{Isothermal titration calorimetry (ITC)}

To measure the binding affinity of Hip to Hsp70 under various conditions, ITC experiments were performed with a MicroCal VP-ITC at $22{ }^{\circ} \mathrm{C}$. All of the reactant solutions were dialyzed against $20 \mathrm{mM}$ HEPES-KOH pH 7.4 and $100 \mathrm{mM} \mathrm{KCl}$ buffer at $4{ }^{\circ} \mathrm{C}$ overnight. The samples and some dialysis buffer were centrifuged at $12,000 \mathrm{rpm}$ and $20{ }^{\circ} \mathrm{C}$ for degassing and removal 
of particles. The sample cell chamber was rinsed with degassed buffer, then around $1.8 \mathrm{ml}$ Hsp70 or Hsp70N (164 - 214 $\mu \mathrm{M})$ was carefully injected into the chamber using a syringe with a long needle until liquid became visible in the aperture. Around $320 \mu 1$ Hip variant concentrate $(1.38-1.76 \mathrm{mM})$ sample were loaded into the injection syringe so that no air bubbles were trapped inside the syringe. During each injection, an $8 \mu \mathrm{l}$ portion of Hip protein from the syringe was added into the calorimeter chamber containing Hsp70. Injections were applied at 180 s intervals. The data were evaluated with MicroCal Origin software (v7.0), assuming independent binding sites.

\subsubsection{Nucleotide release assay}

To determine the effect of Hip on nucleotide release from Hsp70 and its competition with NEFs, we employed the fluorescent nucleotide analog MABA-ADP, which was enzymatically prepared from MABA-ATP (8-[(4-(N-methyl-anthraniloyl)-amino)butyl]-amino-adenosine-5'triphosphate, sodium salt, Interchim). The assay was performed with a SX.18V stopped-flow instrument (Applied Photophysics, Surrey, UK) as previously described (Gässler et al., 2001). All components were in GFM buffer containing additional 1 mM DTT. MABA-ADP complexes were formed by mixing $2.5 \mu \mathrm{M} H \operatorname{sp} 70 \mathrm{~N}$, Hsp70 or an Hsp70N fusion protein with an equimolar concentration of MABA-ADP, followed by incubation at $30{ }^{\circ} \mathrm{C}$ for $30 \mathrm{~min}$. The nucleotide exchange solutions contained $250 \mu \mathrm{M}$ ATP and protein factors as indicated. To determine the nucleotide release rates, equal volumes of the solutions were mixed at $30{ }^{\circ} \mathrm{C}$ in the stopped-flow apparatus. The release of nucleotide MABA-ADP fluorescence was monitored via the time-dependent decrease in fluorescence (excitation at $360 \mathrm{~nm}$ wavelength, emission cut-off filter $400 \mathrm{~nm}$ ) for $1-500 \mathrm{~s}$ at $30{ }^{\circ} \mathrm{C}$. The curves were fitted and analyzed assuming first order kinetics and a constant drift from bleaching. Rate constants were determined by averaging the kinetic constants from each three repeats in three independent experiments.

\subsubsection{Functional characterization in vivo}

\subsubsection{1 $\beta$-Galactosidase assay}


This $\beta$-Galactosidase assay was performed according to an established protocol (Nelson et al., 2004). The reporter strain S. cerevisiae DSY-1100 (Genotype MATa leu2-112, ura3-1, trp1-1, his3-11, 15 ade2-1, can1-100 GAL SUC2 carrying plasmids pDS-125 (GR/TRP) and pDS-362 (GRE-lacZ/URA)) expresses mammalian Glucocorticoid Receptor (GR) and the lacZ gene under control of a Glucocorticoid Responsive Element (GRE) promoter. This strain was transformed with plasmids p423GPD and p423ADH into which the different Hip constructs had been cloned to test the influence on $\beta$-Galactosidase (lacZ is the gene for $\beta$-Galactosidase) activity. The empty plasmids p423GPD and p423ADH were also transformed as background controls. In the assay, the resultant strains were grown in $2 \mathrm{ml} \mathrm{SC}$ medium lacking uracil and tryptophan at $30{ }^{\circ} \mathrm{C}$ overnight, diluted to $\mathrm{OD}_{600}$ of $0.05-0.10$ in a final volume of $1 \mathrm{ml}$ and grown at $25{ }^{\circ} \mathrm{C}$ and $600 \mathrm{rpm}$ for $90 \mathrm{~min}$. Cell growth was monitored spectrophotometrically to ensure that the cells were in the exponential growth phase. The glucocorticoid hormone Deoxycorticosterone (DOC) was then added to a final concentration of $50 \mathrm{nM}$. Hormone binding is needed for GR activation. 90 minutes after hormone addition, $50 \mu 1$ samples were withdrawn at 15-min intervals, the $\mathrm{OD}_{600}$ determined and an equal volume Gal-Screen chemiluminescence reagent (Applied Biosystems) added. After $2 \mathrm{~h}$ incubation in a dark box, the reactions were analyzed with a luminometer (Berthold LB 9507). The emitted light at the different time points was plotted against the $\mathrm{OD}_{600}$, and the slope was determined by linear regression as a measure of functional $\beta$-galactosidase expression. Cells transformed with $\mathrm{p} 423 \mathrm{GPD}$ and $\mathrm{p} 423 \mathrm{ADH}$ served as background controls. All the experiments were repeated at least three times.

\subsubsection{Protein expression analysis by Western blotting}

To analyze the expression levels of the Hip truncation constructs and Hip mutants in the p423ADH yeast strains, Western blotting of whole cell lysates was performed. The strain carrying the empty vector p423ADH was used as a background control. The overnight cultures were diluted to $\mathrm{OD}_{600} 0.1$ to 0.5 and grown to mid-log phase $\mathrm{OD}_{600} 0.5-1.0$ at $30{ }^{\circ} \mathrm{C}$ in selective medium lacking uracil and tryptophan. Equal amounts corresponding to yeast cell $\mathrm{OD}_{600} 2$ in $1.5 \mathrm{ml}$ were harvested by centrifugation at $8000 \mathrm{rpm}$ for 3 minutes at room temperature (Eppendorf 5417). Cell pellets were subjected to alkaline lysis buffer (Kushnirov, 2000). $10 \mu 1$ of the final samples were loaded on a $12 \%$ SDS-PAGE and further analyzed by Western blotting. 


\section{Results}

In order to dissect Hip into functional modules and to determine their crystal structures, a series of truncation mutants and fusion proteins was generated. The constructs are schematically shown in Figure 14A. The construct boundaries were chosen based on sequence conservation between distantly related Hip homologs (for the respective sequence alignment, see Appendix $8.2)$.

\subsection{Purification of Hip}

All the proteins used in this project were overexpressed in the E. coli BL21 codon plus strain as $\mathrm{N}$-terminal His-tag fusion proteins (Fig. 14A). Immobilized metal ion affinity chromatography (IMAC) of the cleared lysate on Ni-Chelating Sepharose (GE) served as first purification step. Proteins were eluted with a step gradient of increasing imidazole concentration. The purification step was monitored by SDS-PAGE analysis. Samples of whole cells (C), insoluble pellet (P), supernatant (lysate) (SN), flow-through (FT), wash steps (W) and elution fractions (E) were analyzed on SDS-PAGE gels. A representative example of such a Coomassie-stained gel from the purification of HipM, Hip(78-247), is shown in Figure 14B.

A)

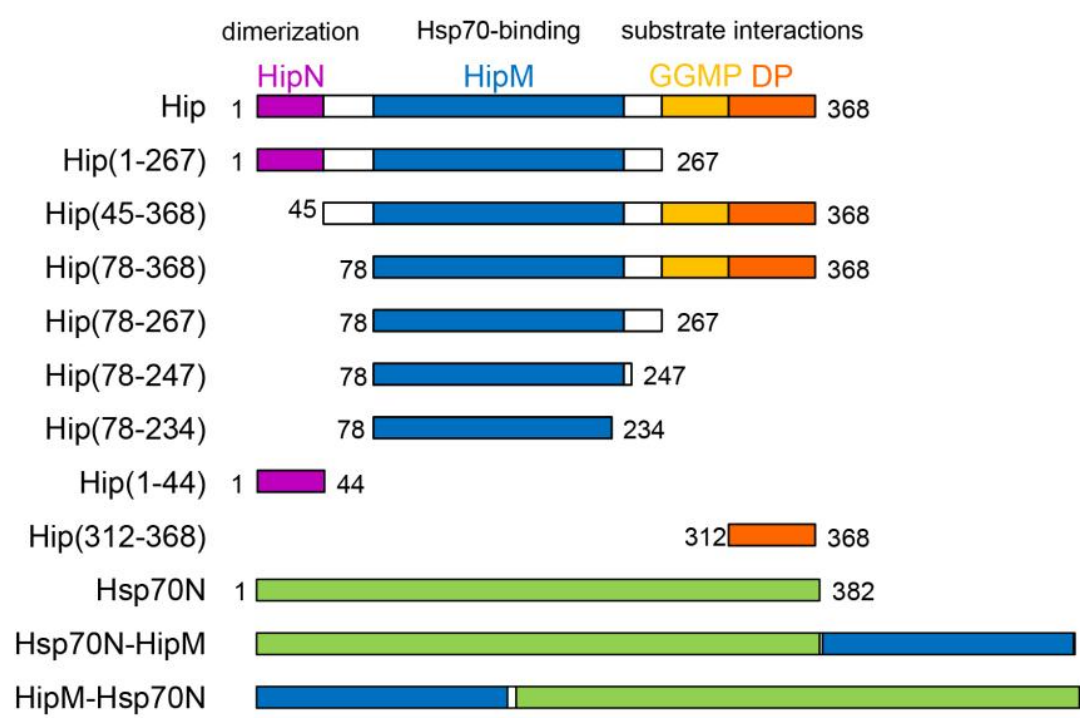


B)

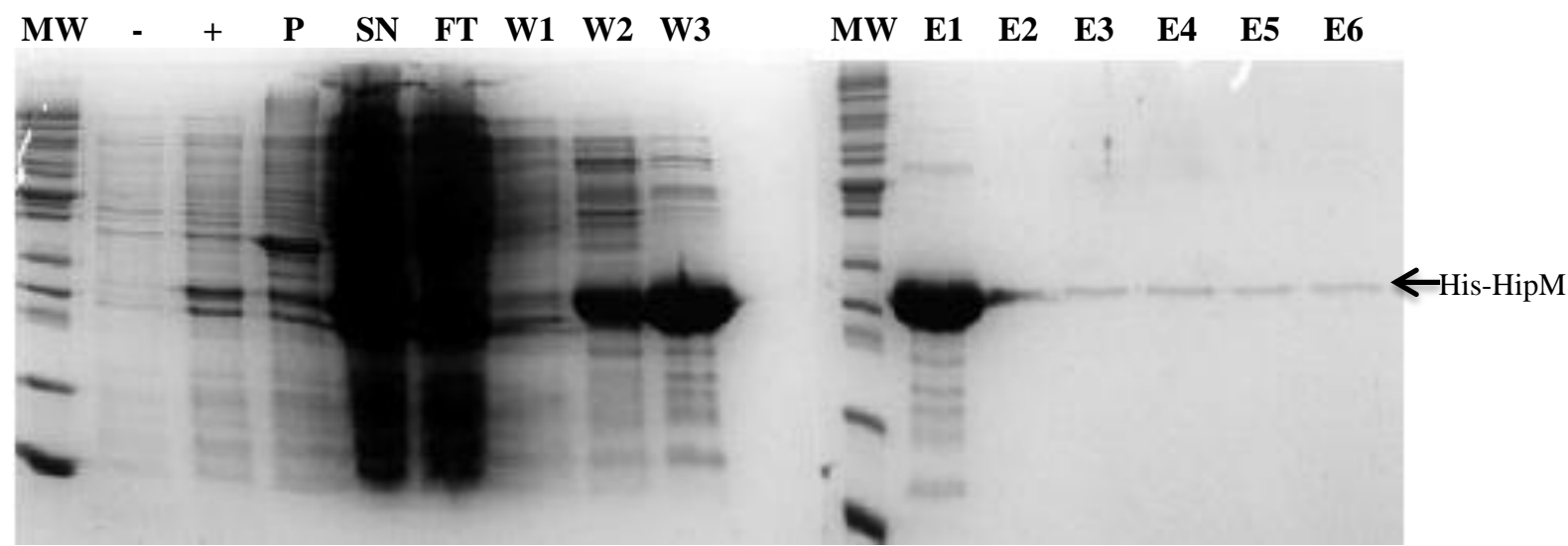

Figure 14: Partial purification of HipM on Ni-Chelating Sepharose.

A) Schematic representation of the Hip constructs analyzed. The dimerization domain (HipN), Hsp70-binding (HipM) and DP domains of rat Hip are shown in purple, blue and orange, respectively. The GGMP repeat segment is indicated in yellow; the acidic linker regions in white. The NBD of human Hsp70 used to construct the Hsp70N-HipM and HipM-Hsp70N fusion proteins is shown in green.

B) $15 \%$ SDS-PAGE gel with samples from a crude purification of His-tagged HipM by nickel-affinity chromatography under native conditions. MW: Molecular weight marker (lanes 1); Whole cell lysate before induction (lane 2); Sample after induction with $0.5 \mathrm{mM}$ IPTG at $20^{\circ} \mathrm{C}$ for 16 hours (lane 3); P: Insoluble pellet (lane 4); SN: Supernatant (lane 5); FT: Flow-through (lane 6); Wash fractions (W1-W3); Elution fractions (E1-E6).

Fractions $\mathrm{W} 3$ and E1 were pooled and subjected to TEV protease digestion performed at $4{ }^{\circ} \mathrm{C}$ overnight to cleave the His-tag segment. To remove imidazole, the protein was subjected to buffer exchange by application to a GE desalting column on the next morning, which was equilibrated with buffer HKM2. The pool of the protein-containing fractions from the desalting column was subsequently applied a second time to the Ni-Chelating Sepharose column to remove the His-tagged TEV protease, the cleaved His-tag and the remaining His-HipM.

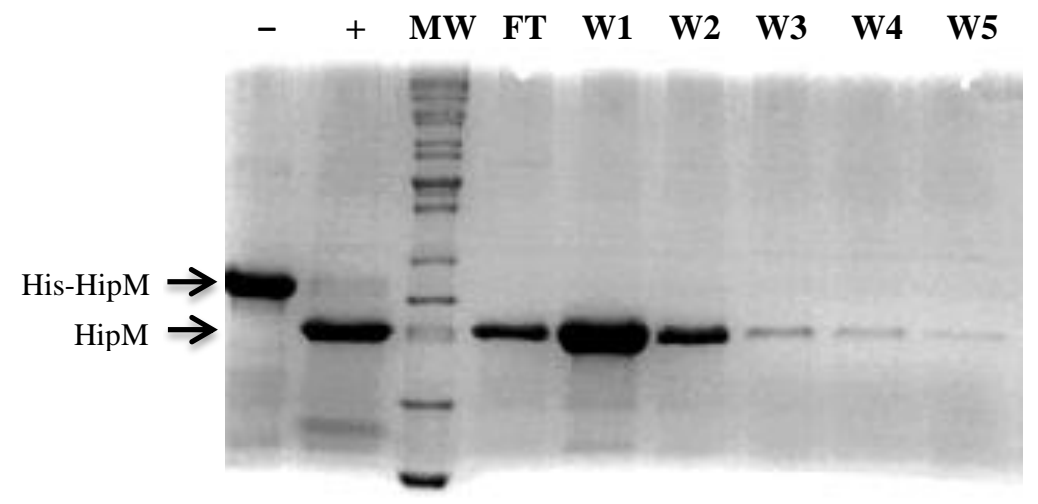

Figure 15: Nickel-affinity chromatography step after TEV protease cleavage in a representative purification of HipM construct. 
15\% SDS-PAGE analysis after TEV cleavage and further purification of HipM with Nickel-affinity chromatography. Sample before TEV cleavage (lane 1); Sample after cleavage over night at $4{ }^{\circ} \mathrm{C}$ (lane 2); Molecular marker (lanes 3); FT: Flow-through (lane 4); W1-W5: wash fractions

After the second run through Ni-Chelating Sepharose, the flow-through and wash fractions were loaded on $15 \%$ SDS-PAGE for checking the cleavage quality and elution efficiency (Fig. 15). It can be seen that almost complete cleavage took place. The His-tagged proteins were removed and HipM protein was eluted in the fractions flow-through, W1 and W2, which were pooled and concentrated to a total volume of $\sim 500 \mu$ l. The concentrate was subjected to size exclusion chromatography on a Superdex-200 column (GE) yielding mono-disperse and pure protein (Fig. 16A). HipM was eluted at an apparent molecular weight of $20 \mathrm{kDa}$, as analyzed by SDS-PAGE (Fig. 16B).

A)

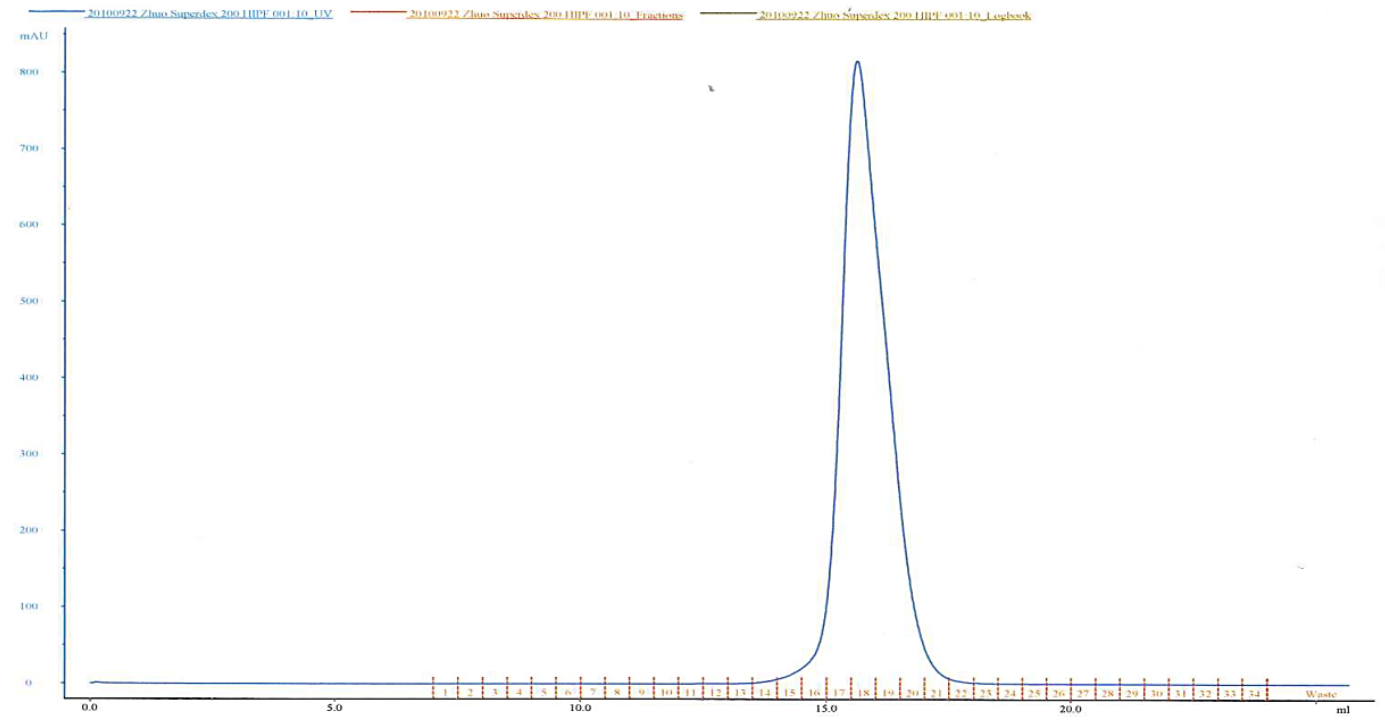

B)

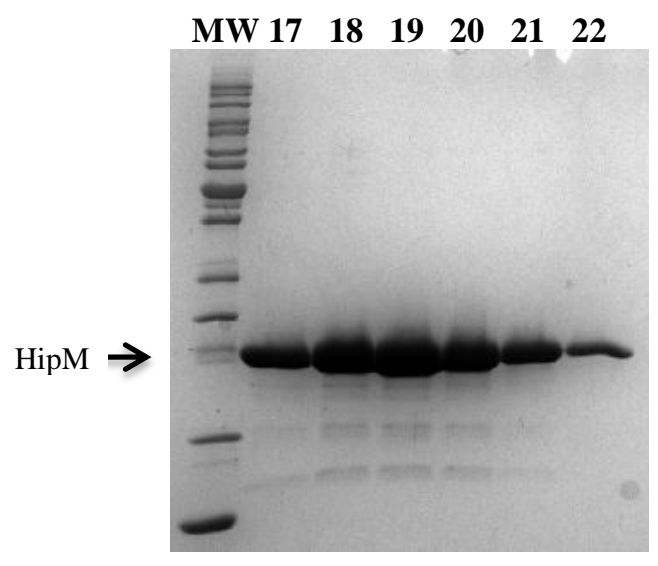


Figure 16: Size exclusion chromatography step of a representative purification.

A) Size exclusion chromatography by using Superdex 200 GL10/300 (GE).

B) $15 \%$ SDS-PAGE gel with fractions from the purification of HipM with size exclusion chromatography. The location of the HipM band is indicated. MW: Molecular weight marker (lane 1); Elution fractions: numbers are indicated above the gel.

After size exclusion chromatography, samples were pooled and concentrated to $~ 18-22 \mathrm{mg} / \mathrm{ml}$ for protein crystallization. The average yield was $\sim 20-30 \mathrm{mg}$ or pure protein from $1 \mathrm{~L} E$. coli BL21 codon plus grown in a shaking culture of LB medium. All the purification steps were performed within 4 days. The purified protein was subsequently stored at $-80^{\circ} \mathrm{C}$.

\subsection{Characterization of the Hip-Hsp70 interaction by isothermal titration calorimetry}

\subsubsection{Selectivity of Hip for the ADP-bound state of Hsp70}

To gain first insight into the mechanism by which Hip modulates the Hsp70 reaction cycle, we used isothermal titration calorimetry (ITC) to quantify Hip binding to Hsp70 and to determine the affinity constant. These experiments were performed with full-length rat Hip and human Hsp70, which shares $97.4 \%$ sequence identity with rat Hsp70. First, we analyzed the interaction of Hip with different conformational states of full-length Hsp70 in the absence of nucleotide (apo) and presence of ADP and ATP.

A)

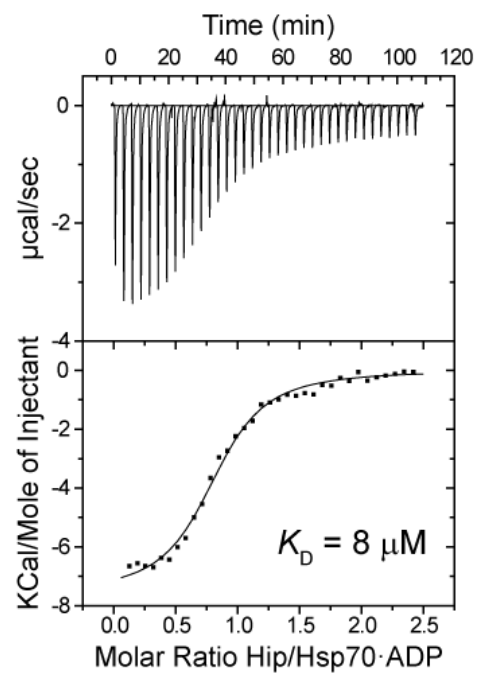

B)

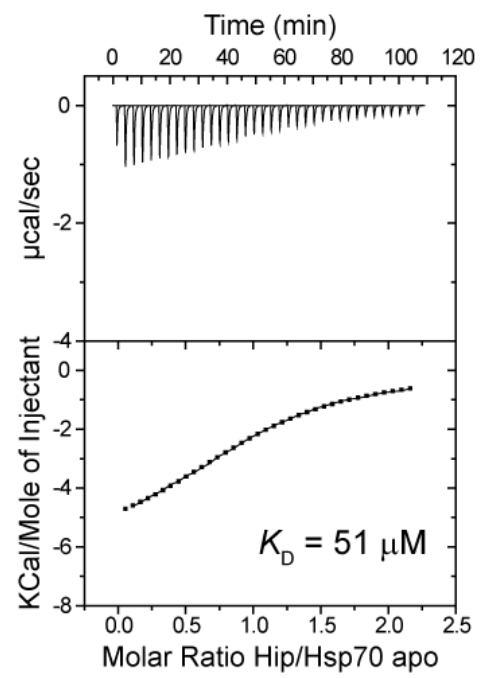

C)

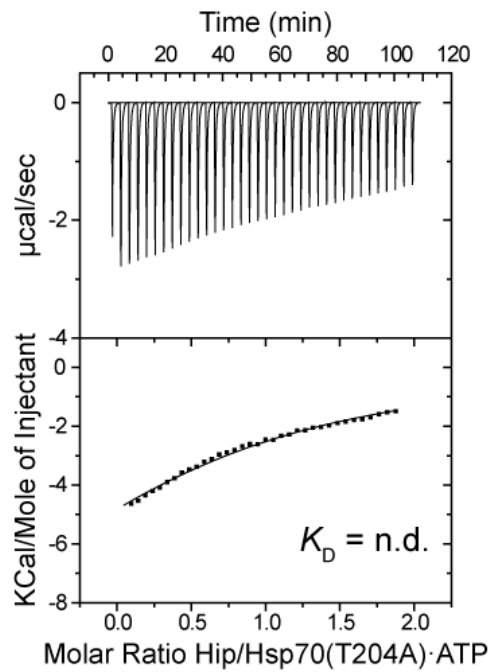


Figure 17: Affinity measurements by isothermal titration calorimetry (ITC) of Hip with Hsp70 in the apo, ADP and ATP-bound conformational states. Upper panel: Energy flow as a result of injection of Hip plotted against time. Lower panel: Heat release per injection plotted against the molar ratio of Hip:Hsp70.

A) Interaction of Hip with $\mathrm{Hsp} 70$ in the ADP-bound state. The experiments were performed at $22{ }^{\circ} \mathrm{C}$ using a buffer of $20 \mathrm{mM}$ HEPES-KOH pH 7.4 containing $100 \mathrm{mM} \mathrm{KCl.} 5 \mathrm{mM} \mathrm{Mg}$-ATP was added when indicated. All data were analyzed assuming that Hip protomers act as independent binding partners.

B) Interaction of Hip with nucleotide-free Hsp70 (apo).

C) Interaction of Hip with Hsp70 in the ATP-induced conformational state. The hydrolysis-defective mutant Hsp70(T204A) was used (Barthel et al., 2001; Kityk et al., 2012). The curve-fitting of the binding parameters for Hsp70(T204A)·ATP data was unstable.

\begin{tabular}{ccccc}
\hline Construct & $\Delta \mathbf{H}\left[\mathbf{c a l m o l}^{-1}\right]$ & $\Delta \mathbf{S ~}^{\left.-\mathbf{c a l m o l}^{-1} \mathbf{K}^{-1}\right]}$ & $\mathbf{N}[\mathbf{m o l a r}$ ratio] & $\mathbf{K}_{\mathbf{D}}[\boldsymbol{\mu M}]$ \\
\hline Hsp70•ADP & -7573 & -2.21 & 0.82 & 8 \\
Hsp70•Apo & -6232 & -1.49 & 1.01 & 51 \\
Hsp70•ATP & -6192 & -0.02 & 1.05 & 26 \\
Hsp70(T204A)•ATP & -11650 & -23.20 & 1 & n.d. \\
Hsp70(D199A)•ATP & -7332 & -2.84 & 1.04 & 16 \\
\hline
\end{tabular}

Table 12: The binding parameters as determined by curve-fitting the ITC experimental data of Hip with Hsp70.

Our data showed that Hip had a strong preference for the ADP-bound form of Hsp70 $\left(K_{D}=8\right.$ $\mu \mathrm{M})$, relative to the nucleotide-free apo state $\left(K_{D}=51 \mu \mathrm{M}\right)($ Fig. $17 \mathrm{~A} \& 17 \mathrm{~B}$, Table 12$)$. We also studied the affinity of Hip for Hsp70-ATP. In an ITC experiment with wild type Hsp70 in the presence of a large excess of ATP, we determined an affinity of $26 \mu \mathrm{M}$, close to the value of ADP-bound Hsp70 (Table 12, data not shown). Because we suspected that Hip might preferentially recognize and stabilize transiently formed ADP-bound Hsp70, we decided to also test ATP-hydrolysis-defective mutants of Hsp70. The Hsp70 mutation D199A is ATPase-inactive and fails to undergo an ATP-induced conformational transition to a more compact state (Buchberger et al., 1995; McCarty and Walker, 1991). Using this mutant we found an affinity for Hip of $16 \mu \mathrm{M}$, suggesting that ATP binding to Hsp70 has only a minor influence on Hip binding (Table 12, data not shown). In DnaK, T199A is a well-characterized mutation, which effectively abolishes ATPase activity, but maintains allosteric coupling. In the crystal structure, this mutant adopts a compact conformation, in which NBD and SBD are closely associated (Kityk et al., 2012; Qi et al., 2013). The co-existence of the "open" and "closed" conformations in the Hsp70 ensemble due to ATP hydrolysis appears to be largely suppressed in this mutant. Based on sequence alignment, the homologous mutation in human Hsp70 is T204A. In the 
presence of ATP, Hip bound with strikingly low affinity to the ATPase-inactive Hsp70 mutant T204A. The titration curve was too shallow for proper quantification (Fig. 17C).

Despite the strong preference of Hip for the ADP-bound state of Hsp70, it should be noted that the affinity to Hsp70.ADP is rather moderate $\left(K_{D}=8 \mu \mathrm{M}\right)$ in comparison to Hsp70 nucleotide exchange factors (NEFs), which all display substantially higher affinity for Hsp70 $\left(K_{D} \approx 0.1\right.$ $\mu \mathrm{M})$. This suggests that the interaction with Hip must be enhanced by additional contacts for allowing Hip to effectively compete with the binding of nucleotide exchange factors (NEFs). For example, interactions of Hip with Hsp70-bound substrate protein may contribute to the strength of association.

Besides the binding affinity, ITC curves may also reveal the binding stoichiometry and the existence of distinct binding sites in the receptor component. The ITC binding curves indicated that one Hip dimer bound two molecules of Hsp70-ADP (Table 12). We found no evidence for any negative interference between the Hsp70 binding sites in the Hip dimer. Thus avidity effects might enhance complex formation of Hip with entities containing multiple Hsp70 molecules.

\subsubsection{Identification of binding site of Hip on Hsp70}

We have demonstrated that Hip selectively binds to full length Hsp70 in the ADP-bound state, which is in line with previous studies (Höhfeld et al., 1995). But Hip was originally identified as binding partner of the NBD of Hsp70 (Höhfeld et al., 1995). We attempted to confirm this by ITC analysis. 
A)

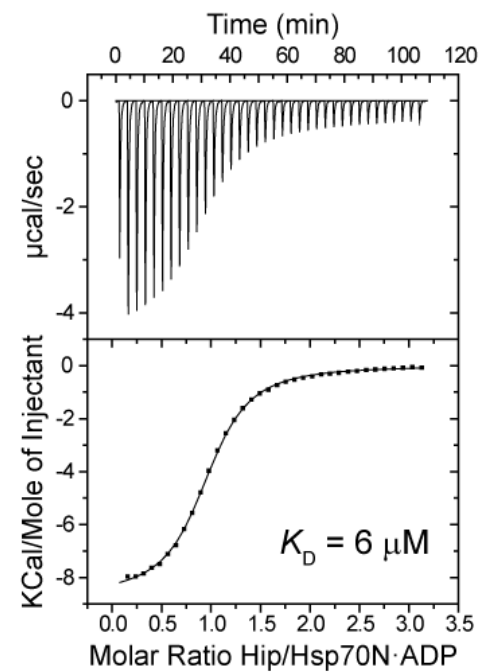

B)

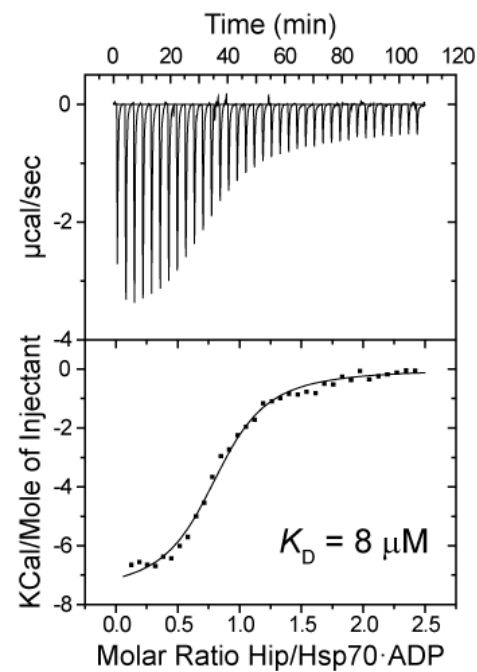

Figure 18: Hip affinity towards the ADP-bound states of Hsp70NBD and full length Hsp70, respectively.

A) ITC titration of full-length Hip with the NBD of Hsp70 (Hsp70N) in the ADP-bound conformational state. The data were analyzed based on the Hip protomer concentration. Prior to dialysis against GF buffer, $1 \mathrm{mM}$ ADP was added to Hsp70N.

B) ITC titration of full-length Hip with full-length Hsp70 in the ADP-bound conformational state.

Based on ITC, the affinity of full-length Hip for the ADP-bound Hsp70N $\left(K_{D}=6 \mu \mathrm{M}\right)$ is essentially the same as that for full-length Hsp70 $\left(K_{D}=8 \mu \mathrm{M}\right)$ (Fig. 18). Thus, the SBD of Hsp70 does not detectably contribute to the interaction with Hip in vitro. It might however influence Hip binding in presence of substrate in vivo.

\subsubsection{Identification of the Hip minimal Hsp70-binding domain}

For the exact identification of the region in Hip responsible for the binding to Hsp70N, truncation constructs were analyzed by ITC (Fig. 19). Höhfeld and coworkers had already found that both the TPR domain and the C-terminally adjacent charged region in Hip are needed for Hsp70 binding (Irmer and Höhfeld, 1997). Residues 107-241 were predicted to form a TPR domain. In a sequence alignment including distantly related Hip homologs (Appendix 8.2), this region is flanked by conserved segments, starting at residue 78 and ending at 267. For these regions, however, no secondary structure was predicted by J-Pred (data not shown), suggesting intrinsically poor ordering, which might be a potential obstacle for crystallization. To our sur- 
prise, the construct encompassing residues 107-267 was not expressed as a soluble protein in $E$. coli (data not shown).

A)

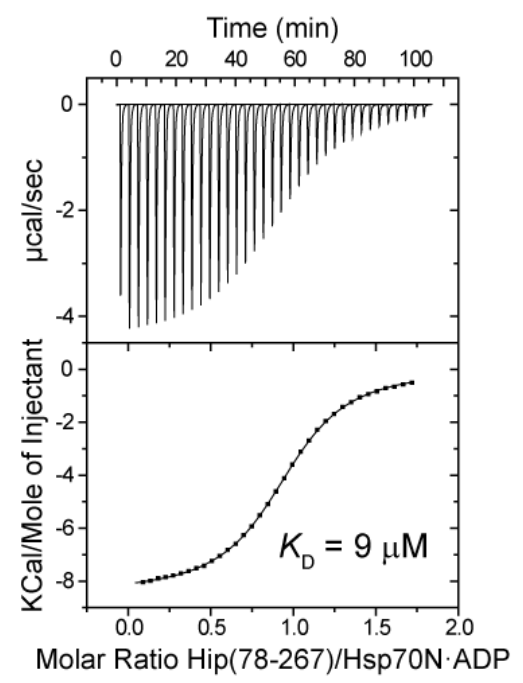

D)

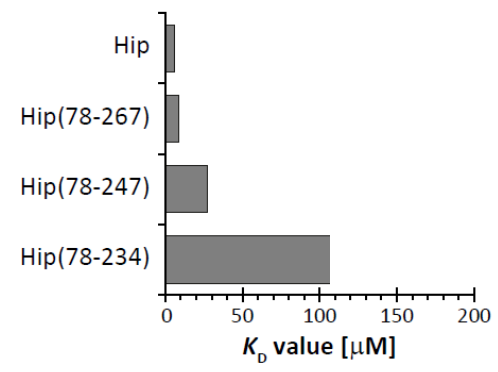

B)

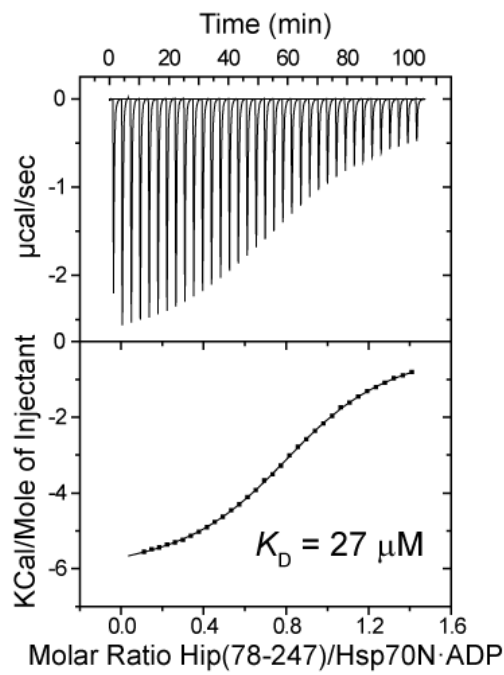

C)

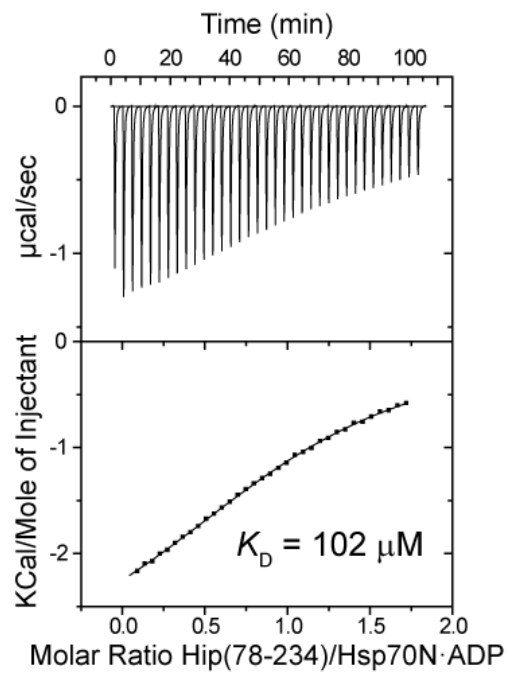

E)

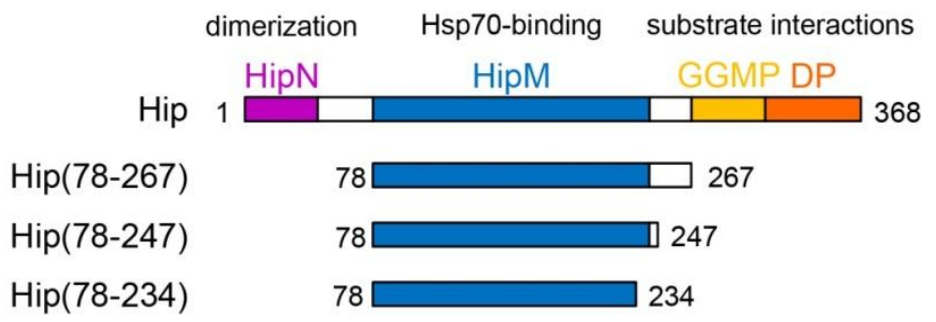

Figure 19: Affinity of Hip truncation constructs towards Hsp70N•ADP.

(A-C) ITC curves for binding of truncation constructs Hip(78-267), Hip(78-247) and Hip(78-234) to Hsp70N • ADP. Prior to dialysis against ITC buffer, $1 \mathrm{mM}$ ADP was added to Hsp70N.

D) Direct comparison of the binding affinities of the truncation constructs towards Hsp70N•ADP.

E) Schematic representation of the Hip truncation constructs analyzed.

Hip(78-267) contains the whole conserved segment and thus the complete Hsp70-binding region as predicted by Höhfeld and coworkers. Using ITC, we found virtually the same affinity to Hsp70N·ADP $\left(K_{D}=9 \mu \mathrm{M}\right)$ as for full-length Hip $\left(K_{D}=8 \mu \mathrm{M}\right)$, suggesting that this segment indeed contains the complete Hsp70-binding region in Hip. C-terminal truncation to Hip(78247) lowered the affinity slightly to $K_{\mathrm{D}}=27 \mu \mathrm{M}$ (Fig. 19B \& 19D). A sharp decrease in affinity 
was found for $\operatorname{Hip}(78-234)$ with $K_{\mathrm{D}}=102 \mu \mathrm{M}$, suggesting that residues 235-247 contribute substantially to Hsp70 binding (Fig. 19C, 19D \& 19E).

\subsection{Hip binding decelerates nucleotide release from Hsp70}

Our ITC analysis demonstrated that Hip preferentially binds to Hsp70 in the ADP-bound state. Hip might thereby also stabilize Hsp70 binding to ADP (Höhfeld et al., 1995). In presence of high ATP concentrations such as in the cytosol, this would only take effect however if ADP dissociation was also slowed down. In order to monitor nucleotide dissociation from Hsp70 in presence of Hip, we used the fluorescent ADP analog MABA-ADP as a reporter. Previous studies have shown that the Hsp70-binding and dissociation properties of MABA-ADP are closely similar to ADP (Brehmer et al., 2004). For the assay, Hsp70 or Hsp70N were incubated with a stoichiometric amount of MABA-ADP to prepare the Hsp70-MABA-ADP complex. Mixing with a great excess of ATP in a stopped-flow apparatus prevents re-binding of MABAADP to Hsp70, resulting in decreased fluorescence (Fig. 20A).

A)

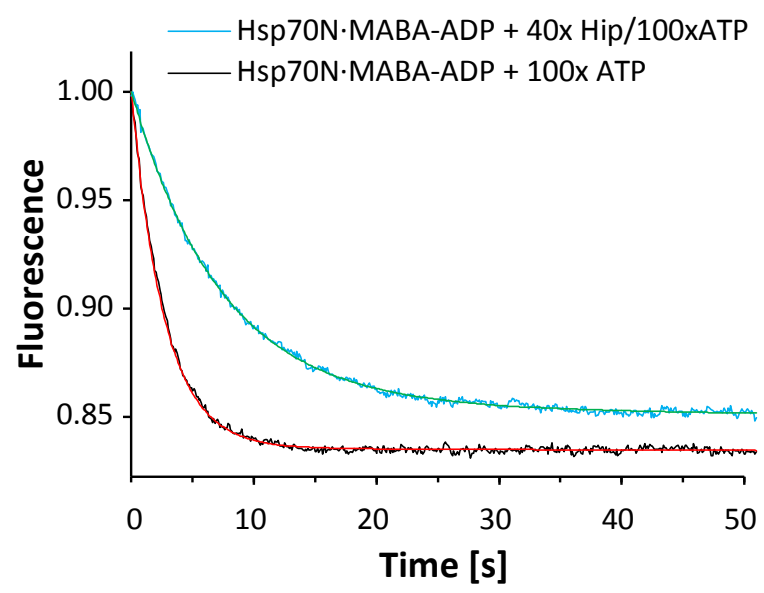


B)

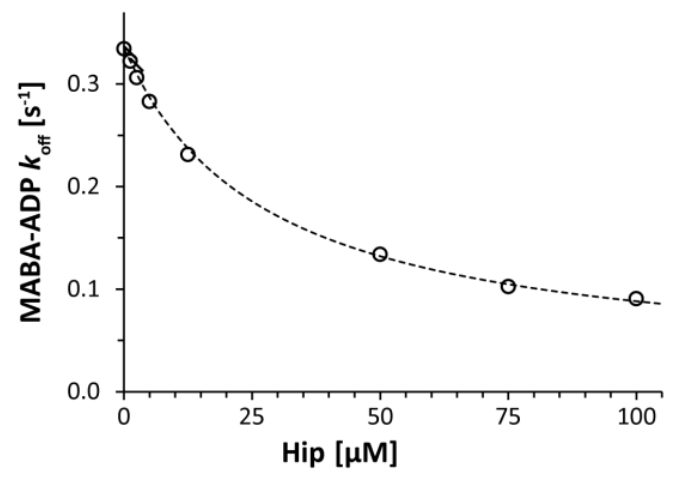

D)

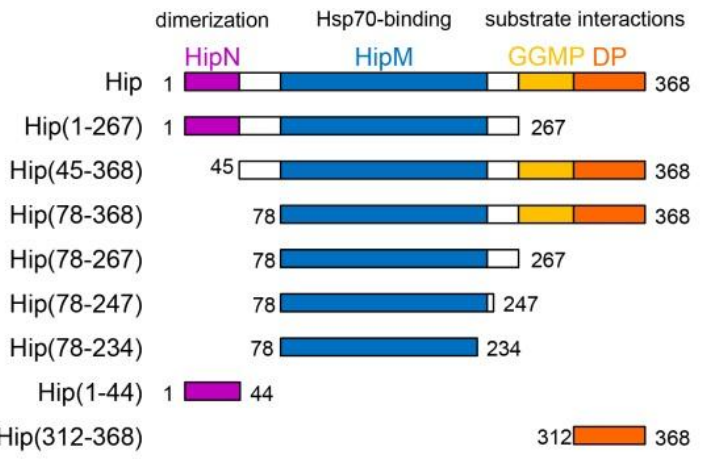

C)

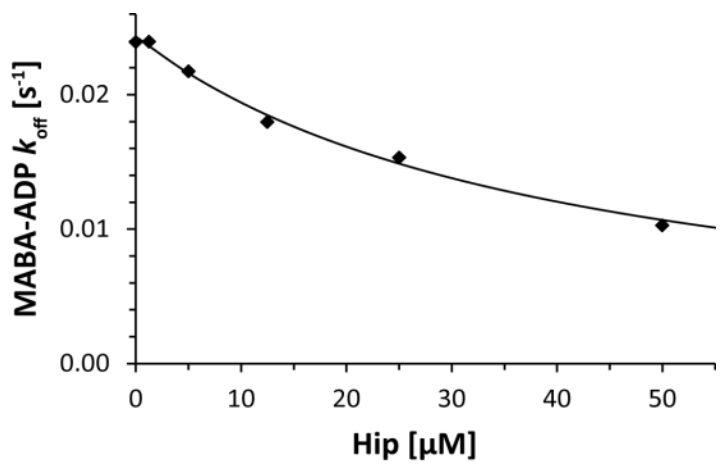

E)

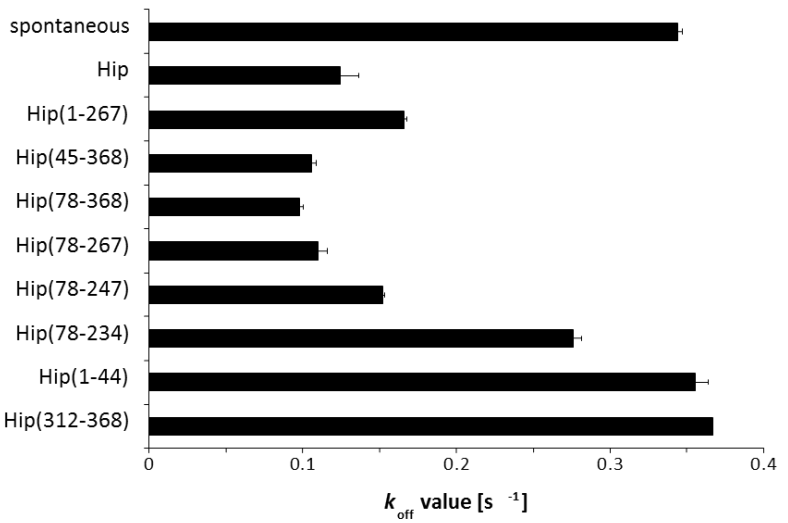

Figure 20: Deceleration of MABA-ADP release from Hsp70N by Hip.

A) Fluorescence traces reflecting the time-dependent dissociation of Hsp70N-bound MABA-ADP. The fluorescent MABA-ADP was incubated for $30 \mathrm{~min}$ with $2.5 \mu \mathrm{M} \mathrm{Hsp} 70 \mathrm{~N}$ at $30^{\circ} \mathrm{C}$, and then mixed in a stopped-flow apparatus with buffer containing $250 \mu \mathrm{M}$ ATP either with or without $100 \mu \mathrm{M}$ of Hip protein (i.e., 40-fold excess over Hsp70N). The fluorescence traces were curve-fitted using a model assuming single-exponential decay and a linear drift to account for bleaching. The red line is the spontaneous release of MABA-ADP from Hsp70N. The blue line is in the presence of $100 \mu \mathrm{M}$ of Hip.

B) Hip-mediated attenuation of MABA-ADP dissociation from Hsp70N in buffer without phosphate. For determining the concentration dependence, the Hip concentration in the ATP solution was varied. Otherwise the solution was as described above. Apparent $k_{\text {off }}$ rates were determined from curve-fitting the exponential decay of MABA-ADP fluorescence and plotted against the final Hip concentration.

C) Hip-mediated attenuation of MABA-ADP dissociation from Hsp70N in buffer containing $10 \mathrm{mM}$ phosphate.

D) Schematic representation of the Hip constructs analyzed. The dimerization domain (HipN), Hsp70-binding (HipM) and DP domains of rat Hip are shown in purple, blue and orange, respectively. The GGMP repeat segment is indicated in yellow; the acidic linker regions in white.

E) MABA-ADP dissociation as a proxy for estimating the affinity of different Hip truncation constructs towards Hsp70. Nucleotide dissociation rates from Hsp70N were measured in presence of each of the Hip truncation constructs. The Hsp70N-MABA-ADP complex was mixed with buffer containing $250 \mu \mathrm{M}$ ATP and $100 \mu \mathrm{M}$ of the respective Hip construct. Apparent $k_{\text {off }}$ rates were determined from curve-fitting the exponential decay 
of MABA-ADP fluorescence. The averages and standard deviations from three independent experiments are shown.

We first investigated the dependence of the MABA-ADP dissociation rate on the Hip concentration under condition of the release assay. In the absence of Hip, the spontaneous MABAADP off-rate was $\sim 0.34 \mathrm{~s}^{-1}$ (red line, Fig. 20A), similar to the ADP dissociation rate for the bacterial homolog of Hsp70, DnaK (Gässler et al., 2001). At an equimolar concentration relative to Hsp70N, Hip lowered the ADP-dissociation rate only marginally, in line with its modest affinity to Hsp70N (Fig. 20B). However, a substantial decrease of $k_{\text {off }}\left(\sim 0.13 \mathrm{~s}^{-1}\right)$ was observed upon the addition of full length Hip in 40-fold excess over Hsp70N (blue line, Fig. 20A), indicating that Hip can stabilize Hsp70 in the ADP bound state.

The ADP release rate from eukaryotic Hsp70 was reported to be greatly lowered in the presence of inorganic phosphate, a component of the eukaryotic cytosol (Brehmer et al., 2001). We suspected that Hip and phosphate might have a multiplicative effect on the dissociation rate. In presence of $10 \mathrm{mM}$ inorganic phosphate, a possible physiological concentration, the spontaneous MABA-ADP off-rate was lowered by a factor of $\sim 15$. However, in relative terms the dependence on Hip concentration did not change substantially in presence of phosphate ( $60 \%$ decrease at $50 \mu \mathrm{M}$ Hip and $1.25 \mu \mathrm{M}$ Hsp70N), arguing against a synergistic effect of Hip and phosphate (Fig. 20C).

Next, we used the MABA-ADP dissociation from Hsp70N as a functional assay for our Hip truncation constructs. To get a robust signal, a 40-fold excess of the respective construct over Hsp70N•MABA-ADP was used (Fig. 20D \& 20E).

The N-terminal dimerization domain Hip(1-44) and C-terminal DP repeat region Hip(312-368) alone did not change the MABA-ADP dissociation rate from Hsp70N, consistent with Hsp70 exclusively binding to the middle segment of Hip. Moreover, the dissociation rates in the presence of full-length Hip(1-368) and the C-terminally truncated construct Hip(1-267) were closely similar, suggesting that the C-terminal GGMP motifs and the DP repeat regions do not contribute to Hsp70-binding (Fig. 20D \& 20E). When we compared the off-rates in presence of dimeric full-length Hip(1-368) with monomeric Hip(45-368) and Hip(78-368), we found a 
slightly increased stabilizing effect with the N-terminal truncation mutants, indicating that dimerization might disturb the interaction with Hsp70 to some degree.

Hip(78-247) and Hip(78-267) slowed MABA-ADP dissociation from Hsp70N to similar extents, consistent with our ITC affinity data. Compared to Hip(78-247) and Hip(78-267), the shorter construct Hip(78-234) had a substantially lower activity, confirming that the functional MABA-ADP dissociation assay can be used as a proxy for affinity.

Taken together, the ITC experiments demonstrated that Hip has a clear preference for the Hsp70•ADP complex. In addition, the NBD of Hsp70 is sufficient for Hip binding. Consistent with the loose domain tethering in the structural model of the ADP complex of Hsp70, the SBD of Hsp70 is neutral towards Hip binding. Moreover, the ability of Hip truncation mutants to attenuate MABA-ADP release from Hsp70N correlated with their binding affinity to Hsp70NADP. The investigation of Hip truncation mutants revealed that both conserved flanking regions to the TPR domain are required for the interaction with Hsp70.

\subsection{Nucleotide release is virtually abolished in a Hip-Hsp70 fusion protein}

Even at a high 40-fold excess of Hip only a partial reduction of the MABA-ADP dissociation rate from Hsp70 was observed in vitro. To estimate the maximal effect of Hip on nucleotide dissociation under saturation conditions, we generated two fusion proteins of HipM and Hsp70N differing in the sequence of the domain segments, HipM-Hsp70N and Hsp70N-HipM, respectively (Fig. 21A).

A)
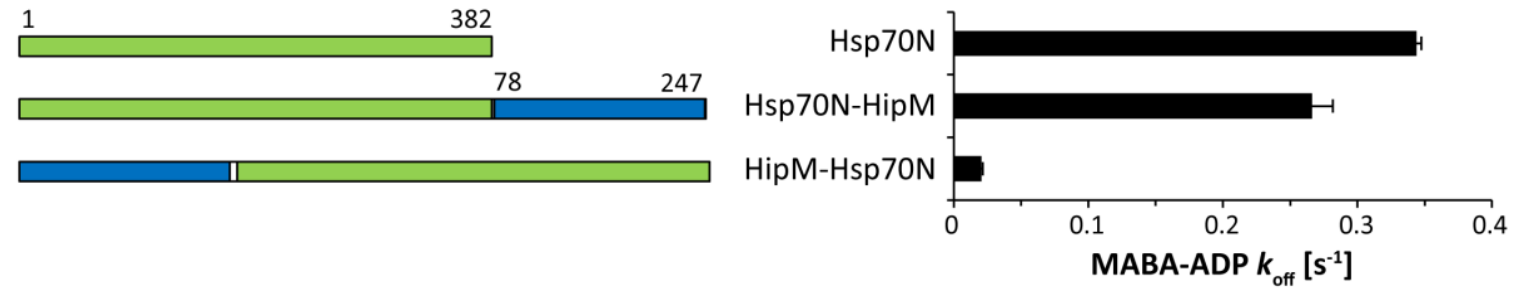
B)

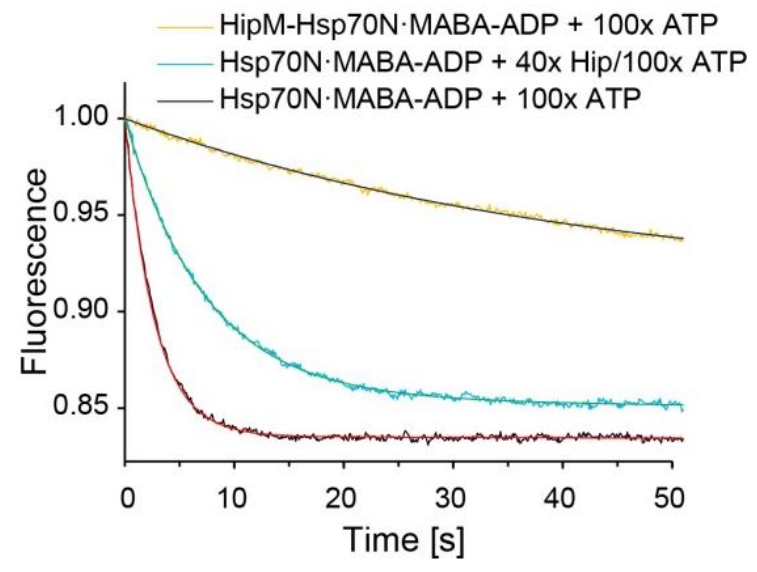

Figure 21: Analysis of the HipM-Hsp70N fusion proteins with the MABA-ADP dissociation assay.

A) Left panel: Schematic representation of the fusion protein constructs. The NBD of human Hsp70 is shown in green; HipM in blue. Right panel: MABA-ADP dissociation rates from the fusion proteins as determined by curve-fitting.

B) Fluorescence traces reflecting the time-dependent dissociation of MABA-ADP. The fluorescent MABAADP was incubated with $2.5 \mu \mathrm{M}$ of the respective protein at $30{ }^{\circ} \mathrm{C}$ for $30 \mathrm{~min}$, and then mixed in a stoppedflow apparatus with buffer containing $250 \mu \mathrm{M}$ ATP. The fluorescence traces were curve-fitted using a model assuming single-exponential decay and a linear drift to account for bleaching. The yellow line was recorded with the HipM-Hsp70N fusion protein. For comparison, the spontaneous dissociation from Hsp70N and the triggered dissociation in presence of a 40 -fold excess of Hip are shown in red and blue (same as in Figure 20A).

In the HipM-Hsp70N fusion construct, the spontaneous MABA-ADP dissociation rate was strongly reduced to a $k_{\text {off }}$ value of $0.02 \mathrm{~s}^{-1}$, indicating a functional intramolecular interface formed between the domains (Fig. 21A, and yellow line in Fig. 21B). This demonstrates that there is ample room for enhancement of the functional effect of Hip by additional interactions stabilizing the Hip:Hsp70 complex. Conversely, the reversed Hsp70N-HipM showed a $k_{\text {off }}$ value close to the spontaneous off-rate, indicating that this fusion protein failed to form a functional intramolecular interface in this orientation (Fig. 21A).

\subsection{Structural analysis of Hip}

\subsubsection{Hip domain structure}

We delineated the probable domain boundaries in rat Hip from the alignment with remote homologs from protists, which became available only after most of the previous functional 
work of Hip had been completed, shown in Appendix 8.2. Including these additional sequences revealed the functional, structured regions as boxes of increased conservation.

Limited proteolysis of full-length Hip gave unclear results (data not shown). The first block of high conservation encompassing the dimerization domain terminates at Pro44 in rat Hip. This is followed by an acidic linker of $~ 30$ amino acids, which is likely to be unstructured. The net negative charge might help to force a Hip dimer into an extended conformation by electrostatic repulsion. The following block of high sequence conservation, residues 78-242 in rat Hip, encompasses additional regions flanking the predicted core TPR domain (residues 107-232) and likely forms a structured middle domain. This is followed by another acidic stretch of $\sim 20$ residues and a variable segment of imperfect repeats of the motif Gly-Gly-(Met/Phe)-Pro (GGMP). At the C-terminus a conserved DP domain is located (residues 312-368 in rat Hip).

\subsubsection{Circular dichroism spectroscopy of Hip domains}

Hip has been proposed to be an almost all $\alpha$-protein (Prapapanich et al., 1998). The secondary structure of a polypeptide chain ( $\alpha$-helix, $\beta$-sheet and random coils) shows a characteristic circular dichroism (CD) spectra. Therefore, we analyzed our Hip truncation constructs using CD spectroscopy to test whether the conserved regions are indeed independently folded domains. The secondary structure content was estimated by deconvolution of the CD spectra with the program CONTIN (Fig. 22).

A)
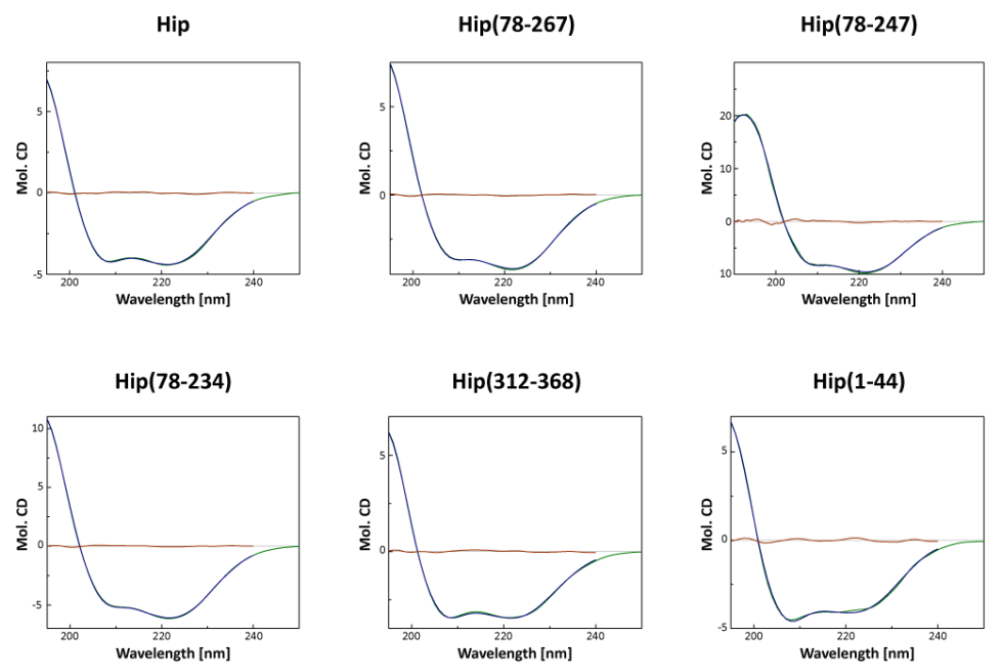
B)

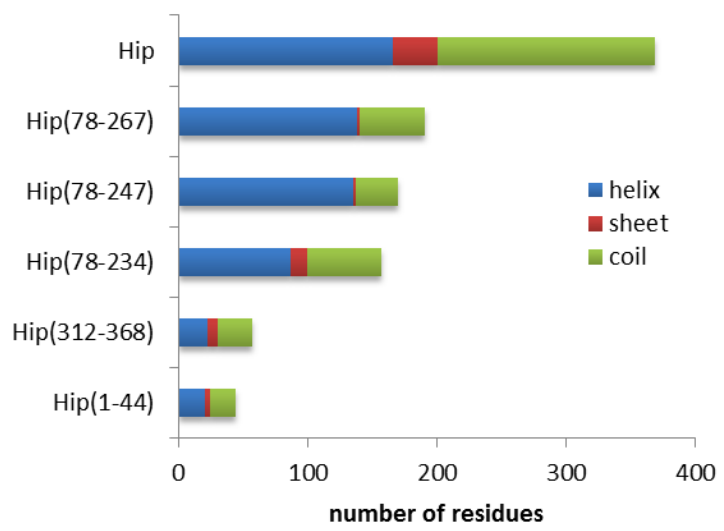

Figure 22: CD Spectroscopic analysis of Hip truncation constructs.

A) CD spectra of Hip constructs. A strong maximum at $195 \mathrm{~nm}$ and two strong minima at 208 and $222 \mathrm{~nm}$ are indicative of $\alpha$-helical structures. A minimum at $218 \mathrm{~nm}$ and maximum at $195 \mathrm{~nm}$ suggest $\beta$-sheet structures. The mean molar ellipticities per residue for indicated constructs are shown in green. Calculated spectra are shown in blue and residual differences in red.

B) Comparison of the putative secondary structure contents, represented as bar graphs. The $\alpha$-helical, $\beta$-sheet and random-coil structure is indicated in blue, red and green, respectively.

The CD spectra indicate that full-length Hip and the analyzed truncation constructs indeed contain a large amount of $\alpha$-helices (Fig. 22A). To compare the truncation constructs with each other, the secondary structure distribution for each construct is shown in Figure 22B. The secondary structure content of full-length Hip is almost identical to the sum of N-terminal dimerization domain Hip(1-44), middle domain Hip(78-247) and the DP domain Hip(312-368), suggesting that these regions indeed represent the structured domains in Hip. The linker regions and the GGMP repeats do not seem to form regular secondary structure.

Furthermore, the analysis of the secondary structure content of the middle domain constructs Hip(78-267), Hip(78-247) and Hip(78-234) revealed that Hip(78-267) and Hip(78-247) had approximately the same amount of $\alpha$-helical structure, suggesting that the $\mathrm{C}$-terminal charged flanking region is unstructured in isolation. Notably, Hip(78-234) had much less helical content, suggesting that residues 235-247 are required for full helix formation in the domain. These findings explain why among the three Hip constructs only Hip(78-247) was successfully crystallized (see section 4.5.4). 
In contrast to Hip(1-44), which was crystallized successfully, we were unable to obtain crystals of the C-terminal Hip(312-368) (data not shown). This suggests that the DP domain of Hip is not stably structured in isolation, and may only assume a defined structure in presence of a bound substrate.

\subsubsection{The DP domain of Hip}

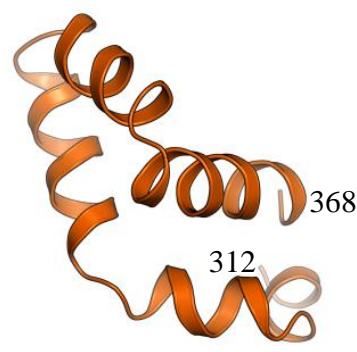

Figure 23: Structure model of the DP domain (312-368) in Hip.

The C-terminal DP domain beginning at residue 312 is quite well-conserved. Analysis of the CD spectra of the domain constructs versus full-length Hip suggested that DP domain of Hip is partially structured (Fig. 22). We further analyzed the domain constructs Hip(1-44), Hip(78234) and Hip(312-368) by one-dimensional 1H-NMR spectroscopy (Rehm et al., 2002) (Zhuo $\mathrm{Li}$, Master thesis). The dispersion of amide resonances indicated that the $\mathrm{N}$-terminal domain was stably structured, whereas this was less so in the spectrum of the C-terminal domain, suggesting that this region was structurally more dynamic (data not shown). Fortunately, the NMR structures of the DP domains in Sti1 (yeast Hop) were solved independently in the meantime (Schmid et al., 2012). Thereby we were able to create a homology model for the respective segment in Hip based on the DP1 domain of Sti1, which is largely consistent with our CD data (Fig. 22).

\subsubsection{Crystallization of the Hsp70-interacting domain of Hip}

Since our initial attempts to crystallize full-length Hip failed, we chose to determine the crystal structures of individual Hip segments (Zhuo Li, Master thesis). At the beginning of the doctoral 
study, we had already solved the structure of the N-terminal dimerization domain of Hip (Zhuo Li, Master thesis). In NMR spectra, the C-terminal DP domain appeared only partially structured. Consistently, crystallization of this segment did not succeed. Therefore, we concentrated on the structure of the Hsp70-binding domain of Hip, which has been predicted to contain a tetratricopeptide repeat (TPR) domain. All three truncation constructs Hip(78-267), Hip(78-247) and Hip(78-234) encompassing this putative TPR domain were tested in crystallization trials based on commercial and in-house factorial screens.

Only the domain construct Hip(78-247) was however successfully crystallized. In the following, this construct is designated HipM - for Hip middle domain. Initial crystals were obtained with two different precipitant compositions: $2.4 \mathrm{M}$ sodium malonate $\mathrm{pH} 7.0$ and $1.4 \mathrm{M}$ tri-sodium citrate, 0.1 M HEPES pH 7.5. These crystals grew in clusters of thin plates. To refine the crystal morphology, we varied the $\mathrm{pH}$ value from 7.0 to 6.0 in the sodium malonate condition. Single, cube-shaped tetragonal crystals appeared after three weeks (Fig. 24A). Conversely, single crystals could only be obtained from Na-malonate $\mathrm{pH} 7.0$ conditions through several rounds of micro-seeding. Unfortunately, the optimization of tri-sodium citrate condition was not successful, even when employing micro-seeding and streak-seeding.
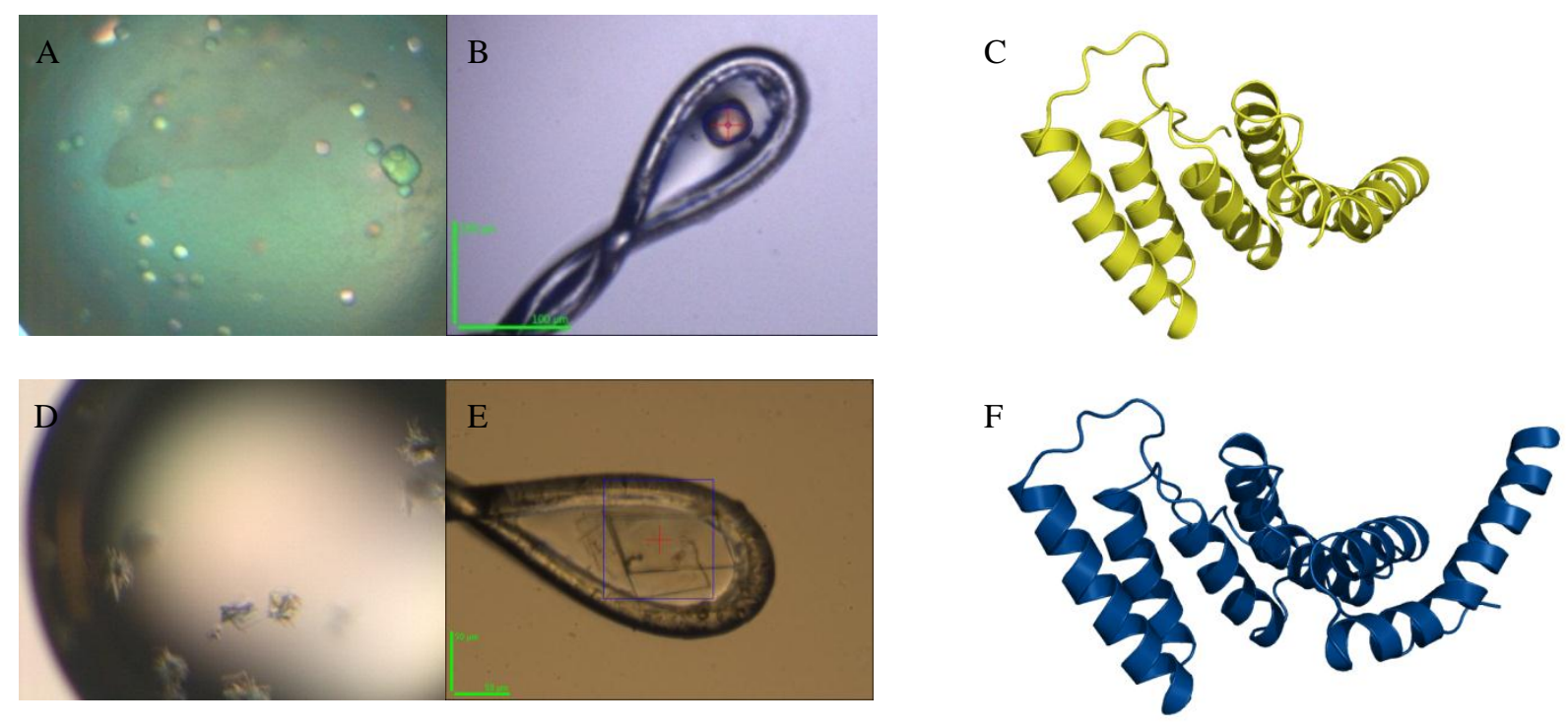

Figure 24: Representative images of Hip(78-247) crystal forms and structures. 
A) Crystals obtained in a hanging-drop setup with $2.1 \mathrm{M} \mathrm{Na}$-malonate $\mathrm{pH} 6.0$ as precipitant at $18{ }^{\circ} \mathrm{C}$.

B) A tetragonal crystal of Hip(78-247) from the Na-malonate condition at $\mathrm{pH} 6.0$ mounted in a cryo loop. The green scalebar corresponds to $100 \mu \mathrm{m}$.

C) Structure of the tetragonal crystal form. Crystal form I was solved by Os-SIRAS at a resolution of $2.6 \AA$. The $\mathrm{R}$ and $\mathrm{R}_{\text {free }}$ values are $18.4 \%$ and $24.8 \%$, respectively.

D) Crystals obtained in a hanging-drop setup with $2.15 \mathrm{M} \mathrm{Na}$-malonate $\mathrm{pH} 7.0$ at $18{ }^{\circ} \mathrm{C}$.

E) Monoclinic plate-shaped crystals of $\mathrm{Hip}(78-247)$ from the Na-malonate condition at $\mathrm{pH} 7.0$ mounted in a cryo loop. The green scalebar corresponds to $50 \mu \mathrm{m}$.

F) Structure of the monoclinic crystal form. Crystal form II was solved by molecular replacement at a resolution of $2.8 \AA$. The $\mathrm{R}$ and $\mathrm{R}_{\text {free }}$ values are $24.6 \%$ and $29.9 \%$, respectively.

\subsubsection{Structure determination and refinement of HipM}

From the construct HipM we obtained two crystal forms, I and II (Fig. 24B \& 24E), with native diffraction to $2.6 \AA$ and $2.8 \AA$, respectively. Table 13 provides an overview of data collection and refinement statistics. The structure of form I was crystallized in space group $\mathrm{P} 4_{3} 2_{1} 2$ with unit cell dimension of $\mathrm{a} \bullet \mathrm{b} \cdot \mathrm{c}=79.86 \AA \cdot 79.86 \AA \cdot 97.31 \AA$. The structure was initially solved by Os-SIRAS at a resolution of $4 \AA$, containing two protein chains per asymmetric unit. The Os substructure was found using the Shelx program package (ShelxC, ShelxD) and contained three Os-atom positions. The heavy atom positions were refined and initial phases were calculated in SHARP (Vonrhein et al., 2007). After further density modifications by Resolve (Terwilliger, 2000), an interpretable map was obtained, which was sufficient to manually build initial models with Coot (Emsley and Cowtan, 2004). The final model building was carried out against a high-resolution native dataset of $2.6 \AA$ resolution. Subsequently, several rounds of refinement were performed with Coot and REFMAC (Murshudov et al., 1997), respectively. The refinement statistics was greatly improved by the application of TLS parameterization in REFMAC. This could indicate that the Hip TPR domains undergo rigid body movements with respect to each other in the crystal lattice. The final structure of form I (Fig. 24C) was refined to a crystallographic R-factor of $18.4 \%\left(\mathrm{R}_{\text {free }} 24.8 \%\right)$.

The structure of the second crystal form II (Fig. 24F) was solved by molecular replacement only after the Hsp70N-HipM crystal structure had been determined (see below), using the HipM segment as a search template. The crystals are twinned, belonging to space group P $2_{1}$ with lattice parameters of $\mathrm{a} \cdot \mathrm{b} \cdot \mathrm{c}=57.29 \AA \cdot 127.00 \AA \cdot 57.31 \AA$, and harbor four copies of HipM per asymmetric unit. The best native diffraction data at $2.8 \AA$ resolution was collected at the European Synchrotron Radiation Facility (ESRF). 
Compared to form I, in which residues 95-212 are ordered, almost the complete HipM construct (residues 81-241 in all chains) was visible in crystal form II. Therefore this crystal form will be discussed first.

\begin{tabular}{|c|c|c|c|c|c|c|}
\hline \multirow{2}{*}{$\begin{array}{l}\text { Construct } \\
\text { Dataset }\end{array}$} & \multicolumn{2}{|c|}{ Hip(1-44) } & \multicolumn{3}{|c|}{ Hip(78-247) } & \multirow{2}{*}{$\begin{array}{c}\text { Hsp70N-Hip } \\
\text { native }\end{array}$} \\
\hline & $\mathrm{EtHgPO}_{4}$ & native & $\mathrm{K}_{2}\left[\mathrm{OsO}_{4}\right]-\mathrm{I}$ & native-I & native-II & \\
\hline $\begin{array}{l}\text { Space group } \\
\text { Cell dimensions }\end{array}$ & $P 2_{1}$ & $P 2_{1}$ & $P 4_{3} 2_{1} 2$ & $P 4_{3} 2_{1} 2$ & $P 2_{1}$ & $P 4_{1}$ \\
\hline a, b, c $(\AA)$ & $\begin{array}{c}28.80,43.89 \\
32.24\end{array}$ & $\begin{array}{c}28.78,43.98 \\
32.21\end{array}$ & $\begin{array}{c}\text { 79.05, 79.05 } \\
96.82\end{array}$ & $\begin{array}{c}79.86,79.86 \\
97.31\end{array}$ & $\begin{array}{c}57.29,127.00 \\
57.31\end{array}$ & $\begin{array}{c}65.60,65.60 \\
141.47\end{array}$ \\
\hline$\alpha, \beta, \gamma\left({ }^{\circ}\right)$ & $90,93.40,90$ & $90,93.44,90$ & $90,90,90$ & $90,90,90$ & $90,105.93,90$ & $90,90,90$ \\
\hline Wavelength (Å) & 1.005 & 0.9000 & 1.140 & 1.005 & 0.9189 & 0.8726 \\
\hline Resolution $(\AA)^{*}$ & $\begin{array}{l}43.90-1.57 \\
(1.67-1.57)\end{array}$ & $\begin{array}{l}43.98-1.1 \\
(1.16-1.1)\end{array}$ & $\begin{array}{l}48.41-4.0 \\
(4.22-4.0)\end{array}$ & $\begin{array}{l}48.84-2.6 \\
(2.74-2.6)\end{array}$ & $\begin{array}{l}45.74-2.8 \\
(2.95-2.8)\end{array}$ & $\begin{array}{l}48.10-2.65 \\
(2.79-2.65)\end{array}$ \\
\hline$R_{\text {merge }} *$ & $0.104(0.375)$ & $0.057(0.323)$ & $0.187(0.432)$ & $0.054(0.338)$ & $0.110(0.427)$ & $0.068(0.491)$ \\
\hline$I / \sigma I^{*}$ & $16.9(6.7)$ & $13.4(4.1)$ & $13.4(6.2)$ & $24.0(5.5)$ & $8.3(2.5)$ & $16.1(2.9)$ \\
\hline Completeness (\%) * & 99.9 (99.7) & $98.6(97.5)$ & $99.8(99.0)$ & $99.5(98.5)$ & $98.7(96.0)$ & $99.9(99.8)$ \\
\hline Redundancy * & $7.3(7.3)$ & $3.6(3.5)$ & $13.1(13.4)$ & $6.3(6.6)$ & $3.1(3.1)$ & $5.1(5.1)$ \\
\hline \multicolumn{7}{|l|}{ Refinement } \\
\hline Resolution ( & - & $20-1.1$ & - & $20-2.6$ & $20-2.8$ & $20-2.7$ \\
\hline No reflections & - & 30476 & - & 9666 & 18235 & 15540 \\
\hline $\begin{array}{l}R_{\text {work }} / R_{\text {free }} \\
\text { Number of atoms }\end{array}$ & - & $0.135 / 0.162$ & - & $0.184 / 0.248$ & $0.246 / 0.299$ & 0.194 / 0.261 \\
\hline Protein & - & 819 & - & 1865 & 4884 & 4243 \\
\hline Ligand/ion & - & 6 & - & 12 & - & 50 \\
\hline $\begin{array}{l}\text { Water } \\
B \text {-factors }\end{array}$ & - & 147 & - & 11 & - & 45 \\
\hline Protein & - & 11.73 & - & 50.14 & 36.26 & 55.25 \\
\hline Ligand/ion & - & 22.63 & - & 61.42 & - & 47.46 \\
\hline $\begin{array}{l}\text { Water } \\
\text { R.m.s. deviations }\end{array}$ & - & 32.10 & - & 30.41 & - & 41.76 \\
\hline Bond length ( & - & 0.015 & - & 0.013 & 0.007 & 0.010 \\
\hline Bond angles $\left({ }^{\circ}\right)$ & - & 1.722 & - & 1.363 & 1.039 & 1.243 \\
\hline
\end{tabular}

Values in parenthesis for outer shell.

Table 13: Summary of Hip data collection and refinement statistics. 


\subsubsection{Structure of HipM}

HipM comprising residues 78-247 is composed of two parts, the N-terminal extension (residues 78-106) and TPR module, consisting of three TPR repeats (residues 107-211) and a capping helix $\alpha 10$ (residues 214-241) (Fig. 25A). Interpretable electron density was visible for residues 81-241 in all four, almost identical copies of HipM in the asymmetric unit of crystal form II (Fig. 25B).

A)

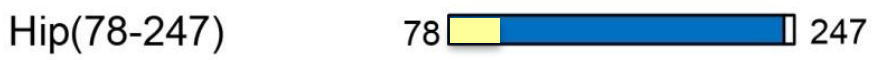

B)
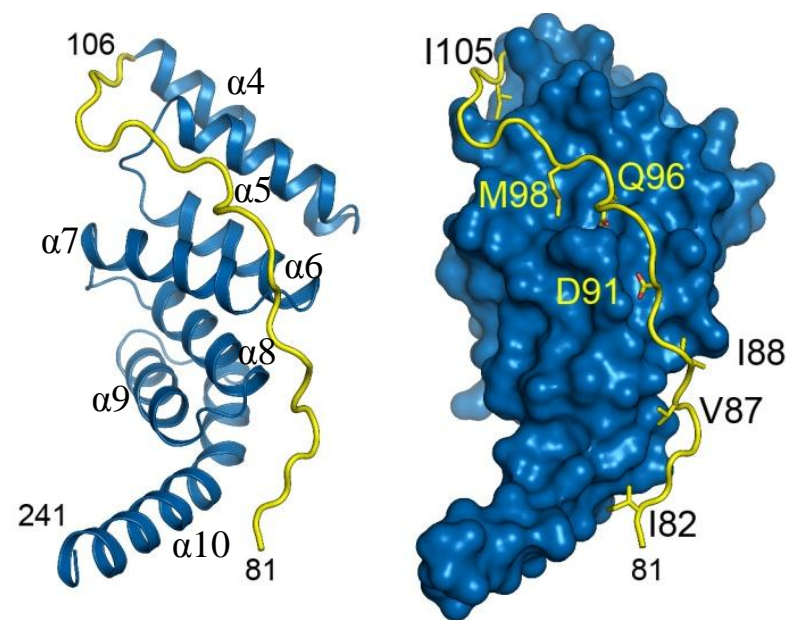

Figure 25: Crystal structure of the Hsp70-binding domain of Hip, HipM.

A) Representation of the architecture of the HipM domain. N-terminal extension (residues 81-106) is highlighted in yellow. The core TPR domain is shown in dark blue.

B) Left panel: Cartoon representation of the overall structure of HipM. Right panel: Detailed view of the interactions between the $\mathrm{N}$-terminal extension and the core TPR domain. The $\mathrm{N}$-terminal extension is shown against the molecular envelope of the TPR domain. Selected side chains in the N-terminal linker extension are labeled and highlighted in stick representation.

The N-terminal extension traverses the helix bundle in the groove between helices $\alpha 5$ and $\alpha 7$ of TPR core. Subsequently, it sneaks along the opposite edge to reach the C-terminal capping helix $\alpha 10$, which protrudes beyond the classical TPR domain structure, bringing $\mathrm{N}$ - and Cterminal segments into close proximity (Fig. 25B). As a result of this arrangement, the negatively charged residues 81-85 are placed in the vicinity of the positively charged C-terminus of 
helix $\alpha 10$. The N-terminal segment is firmly attached to the TPR core by extensive hydrophobic (Ile82, Val87, Ile88 and Ile105) and polar contacts (Asp91, Gln96 and Met98) (Fig. 25B). The buried surface area is $1086 \AA^{2}$, suggesting a stable interaction between the segments in HipM. Consistently, HipM also appeared stable in solution, which was determined by CD spectrometry with a melting point of $56{ }^{\circ} \mathrm{C}$ (data not shown).

Notably, truncation of N-terminal residues 78-106 might destabilize the TPR domain by exposing large hydrophobic surface areas. This is consistent with the insolubility of the construct Hip(107-267). Remarkably, the C-terminal extended final helix $\alpha 10$ might not be stably structured. This is suggested by two lines of evidence. A previous study found that Hip was proteolytically truncated at residues 226 and 237 (Velten et al., 2002). Furthermore, the final helix $\alpha 10$ is not present in crystal form I (Fig. 26A), indicating that it was disordered or cleaved during crystallization. Perhaps prior proteolysis in the $\mathrm{N}$-terminal extension is required to stabilize helix $\alpha 10$. Figure 26 provides an structure overview of the two crystal forms of HipM.

A)

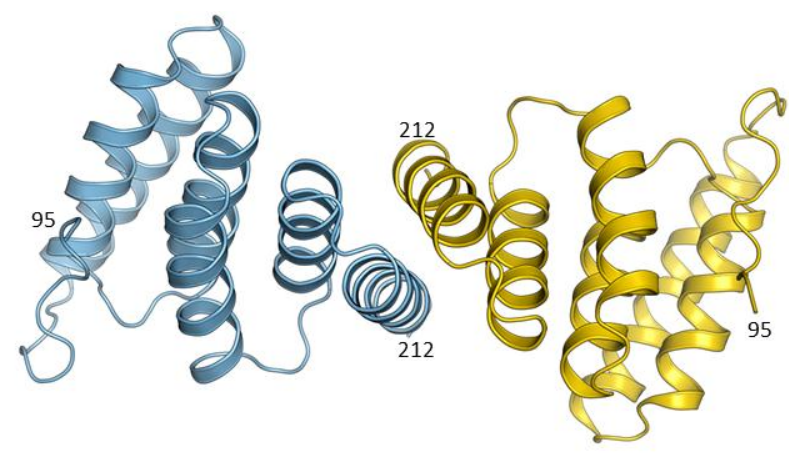

B)

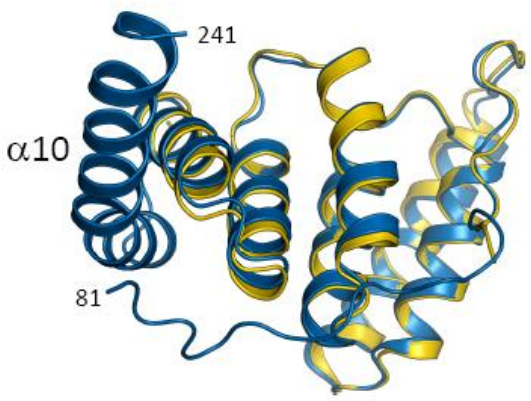

Figure 26: Comparison of the two crystal forms of HipM.

A) Structure of the HipM dimer in crystal form I. The two segments are shown in ribbon representations in light blue and gold, respectively. Residue numbers are indicated for the termini of the ordered segment. 
B) Superposition of the HipM units in crystal forms I and II. For clarity, only one representative chain from each crystal form is shown. The chain from crystal form II is shown in dark blue. The additional helix is indicated as $\alpha 10$.

Form I contained two protein chains per asymmetric unit, forming a symmetrical dimer (Fig. 26A). Only residues 95-212 were ordered in both copies, i.e. density for the capping helix $\alpha 10$ was completely absent. Otherwise, the structured part was closely similar to crystal form I as shown in Figure 26B. Subsequent SDS-PAGE analysis of dissolved crystals revealed an approximate 1:1 mixture of two chain lengths, one similar to full-length HipM (data not shown), suggesting partial proteolytic degradation. The exact composition of the crystals remains therefore unclear.

Size exclusion chromatography gave no indication for dimerization of HipM in solution (Fig. 16A). The buried surface area of $\sim 600 \AA$ at the interface is probably too small for a stable interaction. Two opposed cysteines were found at the interface, however no disulfide bond formation was observed. Therefore, this dimer formation is probably a consequence of crystal packing.

\subsubsection{Small angle X-ray scattering (SAXS)}

Since full-length Hip could not be crystallized, the structural features of Hip as a whole remained unknown. Small angle X-ray scattering (SAXS) analysis offers complementary information about macromolecular folding, conformation and assembly state in solution with resolution range of about $50 \AA$ to $10 \AA$. Together with the solved three-dimensional structures of individual domains of Hip, we attempted to create a model for a complete Hip dimer.

Mainly two parameters can be estimated from SAXS data, the particle radius of gyration $\mathrm{R}_{\mathrm{g}}$ and the maximum linear dimension of the scattering particle $D_{\max } . R_{g}$ can be obtained by curvefitting with the programs Guinier and GNOM using Guinier's law and $\mathrm{D}_{\max }$ can be calculated with GNOM. When the atomic structures are available, the theoretical curves can be calculated from the pdb files by evaluating all the interatomic distances in the structure. In the direct com-

parison of such theoretical curves with SAXS data, the Chi value $\left(\chi^{2}\right)$ is used to estimate the 
agreement between the atomic model with the experimental data from SAXS, which is similar to the R-factor in crystallography by indicating the goodness-of-fit.

Following this methodology, an $\mathrm{R}_{\mathrm{g}}$ of $6.05 \mathrm{~nm}$ was identified for full-length Hip. The respective pair distance distribution function $p(r)$ is shown as insert, suggesting a large $D_{\max }$ of $202 \AA$ (Fig. 27A). These dimensions are consistent with previous observations (Dores-Silva et al., 2012; Velten et al., 2000), suggesting an elongated conformation for the complete $\sim 80 \mathrm{kDa}$ Hip dimer with a maximum extension of $\sim 200 \AA$ (Fig. 27A). The Kratky plot representation of the full-length Hip data indicated that a substantial fraction of Hip is unstructured (Fig. 27B), which is consistent with the secondary structure composition derived from CD spectroscopy.

A)

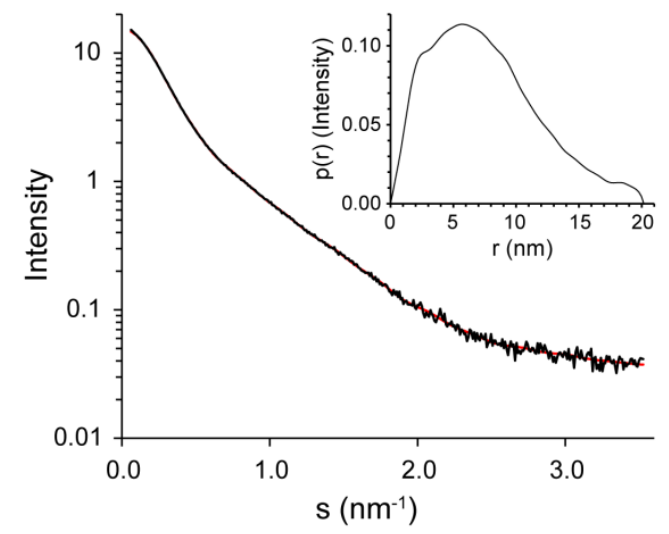

B)

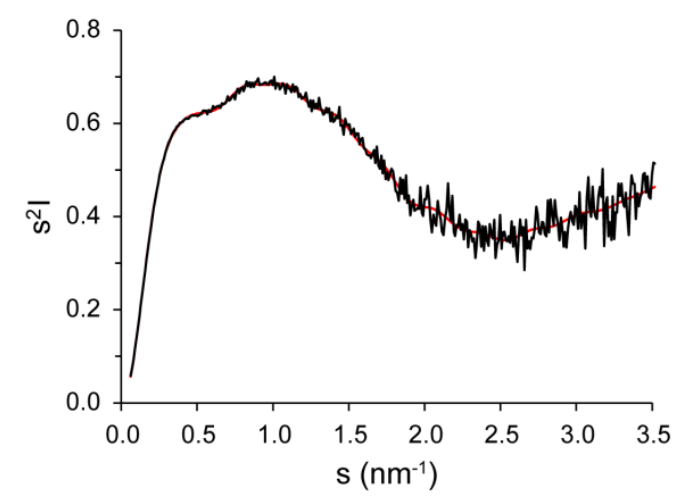

Figure 27: SAXS Structural analysis of the Hip dimer.

A) X-ray scattering curve of Hip at $1.8 \mathrm{mg} / \mathrm{ml}$ in the presence of $20 \mathrm{mM}$ HEPES, $100 \mathrm{mM} \mathrm{KCl}$ and $1 \mathrm{mM} \mathrm{DTT}$, $\mathrm{pH}$ 7.4. The red line indicates the best fit obtained with the indirect Fourier transform method using the program GNOM (Svergun, 1992). The respective pair distance distribution $p(\mathrm{r})$ is shown as insert, suggesting a large $\mathrm{D}_{\max }$ of $202 \AA$ for the $80 \mathrm{kDa}$ particle. The radius of gyration $\left(\mathrm{R}_{\mathrm{g}}\right), 6.05 \mathrm{~nm}$, was determined using the Guinier approximation, as implemented in PRIMUS (Konarev et al., 2003).

B) Kratky plot representation of the scattering data. The high signal of $\mathrm{s}^{2} \cdot \mathrm{I}$ at $\mathrm{s}>2.5 \mathrm{~nm}$ indicates that a substantial fraction of Hip is unstructured.

Besides full-length Hip, two Hip truncation mutants, Hsp70 and the HipM-Hsp70 fusion protein were also studied (data not shown). Table 14 summarizes the key results derived from SAXS data. 


\begin{tabular}{lllllc}
\hline \multicolumn{1}{c}{ Construct } & \multicolumn{2}{c}{ SAXS Scattering curve } & \multicolumn{2}{c}{ Atomic model } & Chi fit $\left(\chi^{2}\right)$ \\
\cline { 2 - 5 } & $\mathbf{R}_{\mathbf{g}}[\mathbf{n m}]$ & $\mathbf{D}_{\max }[\mathbf{n m}]$ & $\mathbf{R}_{\mathbf{g}}[\mathbf{n m}]$ & $\mathbf{D}_{\mathbf{m a x}}[\mathbf{n m}]$ & \\
Hip & $6.05 \pm 0.04$ & 20.18 & - & - & - \\
Hip(1-267) & $5.08 \pm 0.01$ & 17.77 & - & - & - \\
Hip(78-247) & $2.25 \pm 0.01$ & 8.31 & 1.78 & 6.50 & 14.64 \\
Hip(78-234) & $1.98 \pm 0.004$ & 6.33 & 1.68 & 6.02 & 10.78 \\
Hsp70N & $2.44 \pm 0.01$ & 8.39 & 2.10 & 7.17 & 5.197 \\
HipM-Hsp70N & $2.73 \pm 0.01$ & 9.56 & 2.54 & 8.51 & 4.567 \\
\hline
\end{tabular}

Table 14: Comparison of structural data from SAXS and crystallographic analysis

Both Hip(78-247) and Hip(78-234) showed quite high Chi values. One explanation could be that these two constructs might show more open conformations in solution than suggested from the crystal structure of HipM, Hip(78-247). Especially the SAXS data for the functional fusion protein HipM-Hsp70N are in good agreement with the determined Hip:Hsp70 core complex crystal structure (see below). Furthermore, the $\mathrm{D}_{\max }$ of the fusion protein is similar to Hsp $70 \mathrm{~N}$, indicating a compact conformation of this protein in solution.

\subsection{Structural analysis of the Hip:Hsp70 complex}

\subsubsection{Crystallization of the Hip:Hsp70 core complex}

To gain further insight into the mechanism by which Hip stabilizes Hsp70, we determined the crystal structure of Hsp70 in complex with Hip.

Since the individual crystal structures of Hsp70N and the Hsp70-binding domain of Hip, HipM, were known, we first tried to co-crystallize Hsp70N and HipM. ITC experiments had shown that the binding ratio of these two proteins was $1: 1$ with a modest binding affinity $\left(K_{\mathrm{D}}=27\right.$ $\mu \mathrm{M})$. The co-crystallization trials were first performed at a molar ratio of $1: 1$. In order to increase complex formation, we modified the molar ratio of 1:1.2 and 1:1.5, assuming that HipM was less likely to crystallize by itself than Hsp70N, which crystallized readily. Unfortunately, the co-crystallization of Hip with $\mathrm{Hsp} 70 \mathrm{~N}$ failed. The crystals invariably contained only Hsp70N in the apo or ADP-bound form, depending on whether exogenous ADP was added or not (data not shown). To obtain the structure of a Hip:Hsp70 complex, we therefore changed 
our strategy by employing the fusion proteins of HipM and Hsp70N. The proximity of the domains was assumed to enforce intramolecular interactions. As described above, we designed two different types of fusion proteins, the functional HipM-Hsp70N and the rather nonfunctional Hsp70N-HipM (Fig. 21A). While crystals could not be obtained from the HipMHsp70N fusion protein, the reverse construct, Hsp70N-HipM, produced crystals. These appeared as needles in the initial screen with $0.2 \mathrm{M} \mathrm{NH}_{4} \mathrm{I}$ and $20 \%$ PEG 3350 as a precipitant at both $4{ }^{\circ} \mathrm{C}$ and $18{ }^{\circ} \mathrm{C}$ (Fig. 28A \& 28B). To improve crystal quality, the crystallization condition was optimized and micro-seeding was performed at $18{ }^{\circ} \mathrm{C}$. A single rod crystal could be obtained by micro-seeding with $0.2 \mathrm{M} \mathrm{NH} 4 \mathrm{I}$ and $17 \%$ PEG 3350 as precipitant using the hanging drop method (Fig. 28D).
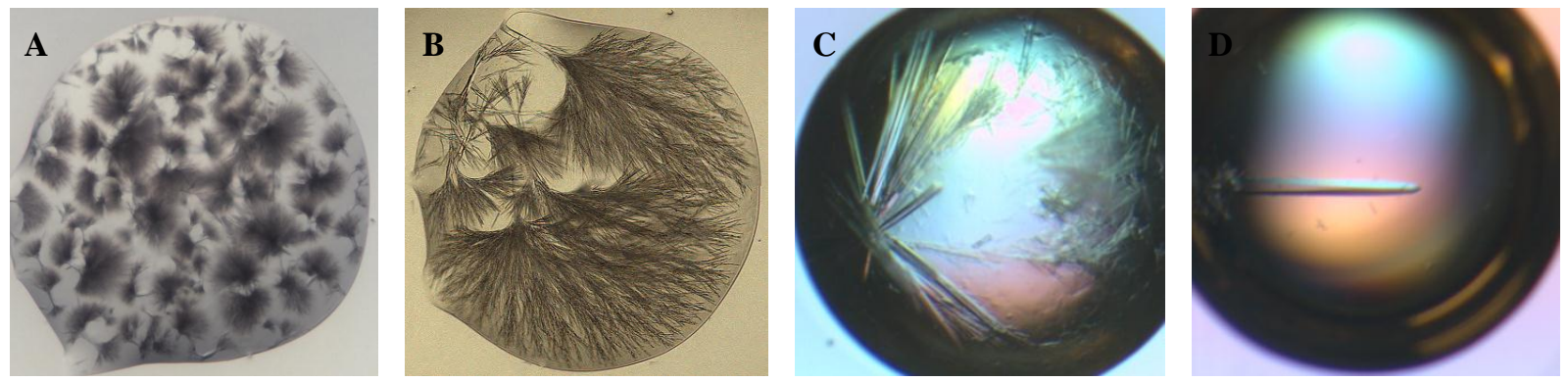

Figure 28: Representative images of the HipM-Hsp70N crystal form.

A) Initial crystals obtained in the sitting-drop setup under the condition using $0.2 \mathrm{M} \mathrm{NH}_{4} \mathrm{I}$ and $20 \%$ PEG 3350 at $4{ }^{\circ} \mathrm{C}$

B) Initial crystals obtained in the sitting-drop setup under the same conditions at $18{ }^{\circ} \mathrm{C}$

C) Crystals obtained in the hanging-drop setup with of $0.2 \mathrm{M} \mathrm{NH}_{4} \mathrm{I}$ and $17 \%$ PEG 3350 at $18{ }^{\circ} \mathrm{C}$

D) A single crystal obtained after seeding in the hanging-drop setup using $0.2 \mathrm{M} \mathrm{NH}_{4} \mathrm{I}$ and $17 \%$ PEG 3350 as a precipitant.

\subsubsection{Structure determination of the Hsp70-Hip fusion protein}

X-ray diffraction data were collected from the single rod crystal to $2.7 \AA$ resolution. The analysis showed that the space group is $\mathrm{P} 4{ }_{1}$ with lattice parameters of $\mathrm{a} \cdot \mathrm{b} \cdot \mathrm{c}=65.5 \AA \cdot 65.5 \AA$ • $141.47 \AA$ (Table 13). The structure of the Hsp70N-HipM fusion protein was solved by molecular replacement using the program Molrep. Hereby, the Hsp70(1-393)-ADP complex and the HipM monomer form I were used as the searching templates. Hsp70N-HipM was almost fully defined in the density including the human Hsp70 (residues 1-382), a short linker and rat Hip (residues 78-243), as well as bound ADP. $\mathrm{P}_{\mathrm{i}} \cdot \mathrm{Mg}^{2+} \cdot 17$ structurally defined iodine ions from the 
precipitant solution were confirmed with the anomalous signal in a dataset collected from a different crystal at $2.0 \AA$ wavelength. Several of these iodide ions are located at the domaindomain interface and at crystal contacts, presumably stabilizing crystal packing interactions (Fig. 29A \& 29B). Therefore, the presence of $200 \mathrm{mM} \mathrm{NH}_{4} \mathrm{I}$ in the crystallization condition presumably plays an important role in crystal stabilization.
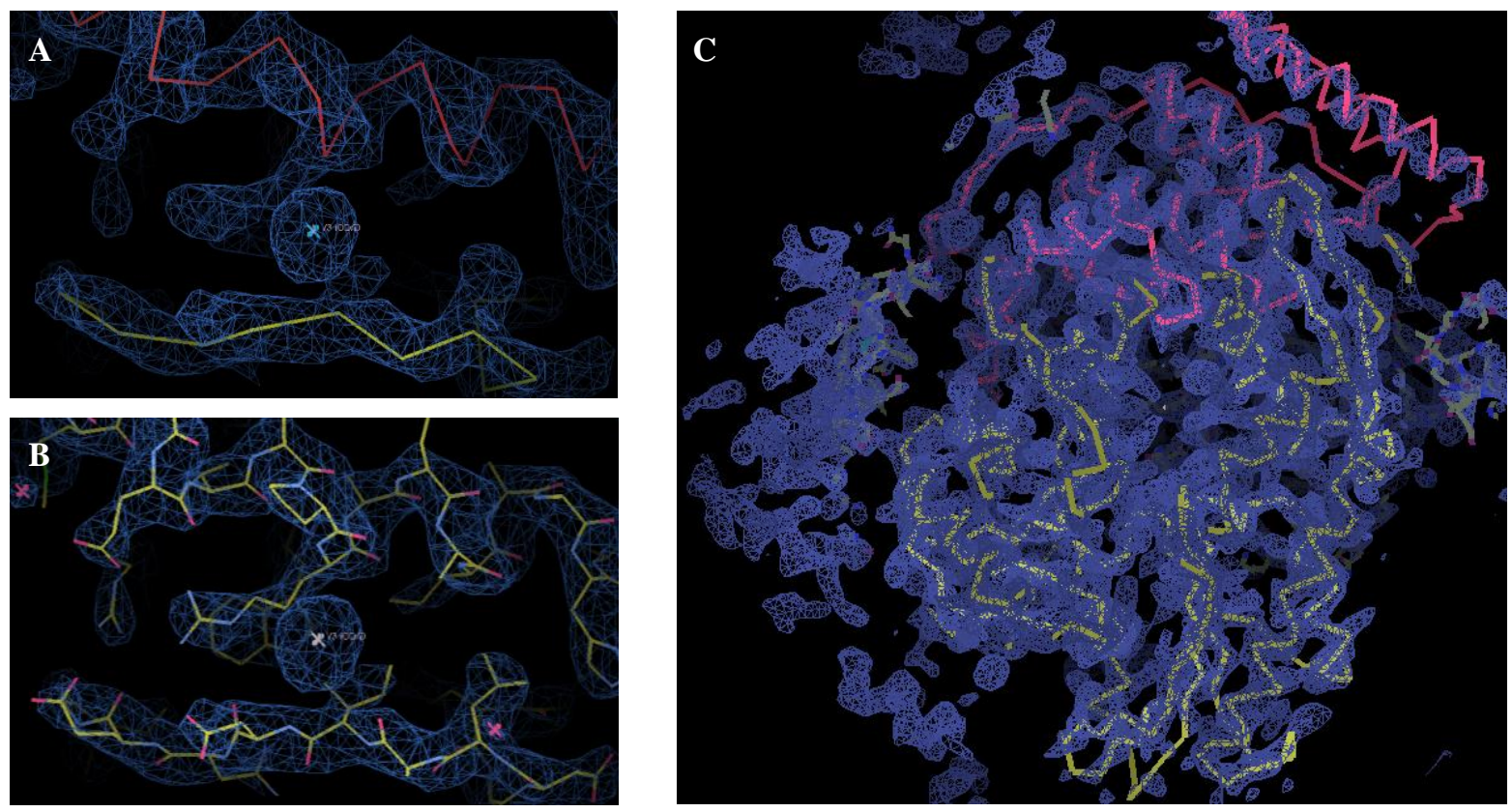

Figure 29: Electron density map of the fusion protein Hsp70N-HipM.

A) Representative portion of an initial electron density map. The intermolecular interface of HipM (red), Hsp70N (yellow) and resolved iodine ion shown in cross.

B) The final structural model is overlaid and shown as color coded stick representation.

C) The representative intermolecular interface of the fusion protein in the initial electron density map.

The complex structure was refined to an $\mathrm{R}$-factor of $19.4 \%\left(\mathrm{R}_{\text {free }}\right.$ of $\left.26.1 \%\right)$. All models were built interactively using the program Coot. Refinement was performed with the program REFMAC. Residues (apart from glycine) facing solvent channels were modeled as alanine, when side chain density was not discernible. Interestingly, besides the intramolecular interface within the fusion protein Hsp70N-HipM, the likely intermolecular interface between Hsp70N and HipM could be observed in the electron density map (Fig. 29C). 


\subsubsection{Two interaction interfaces between Hip and Hsp70}

In the electron density map, two plausible interfaces between Hsp70N and HipM domains were observed. Figure 30A and 30B show the two different interfaces between Hip and Hsp70 together with the surface conservation in Hip at the respective region.

A)

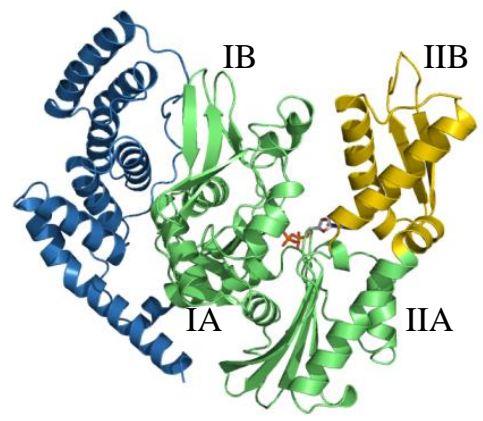

D)

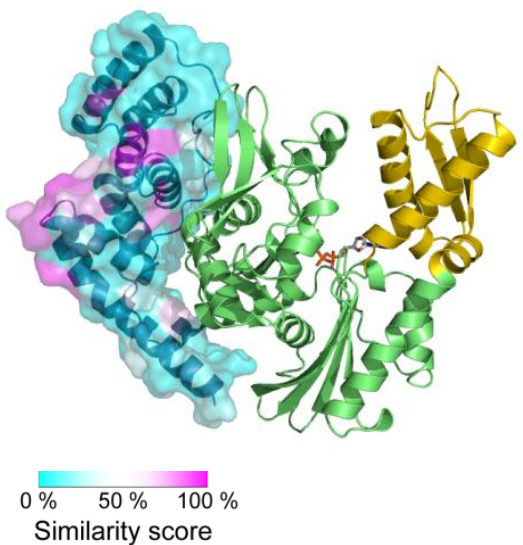

B)

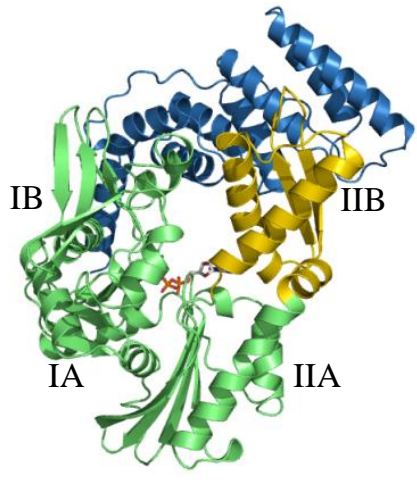

E)

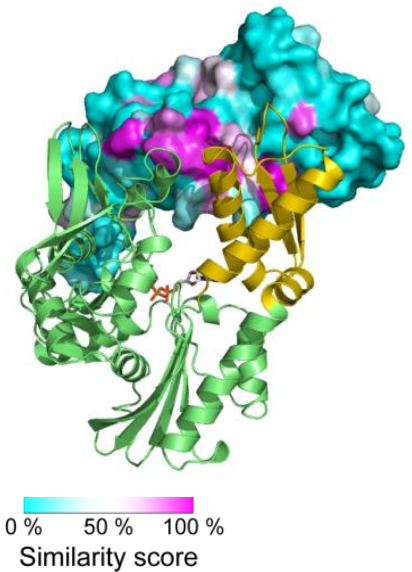

C)

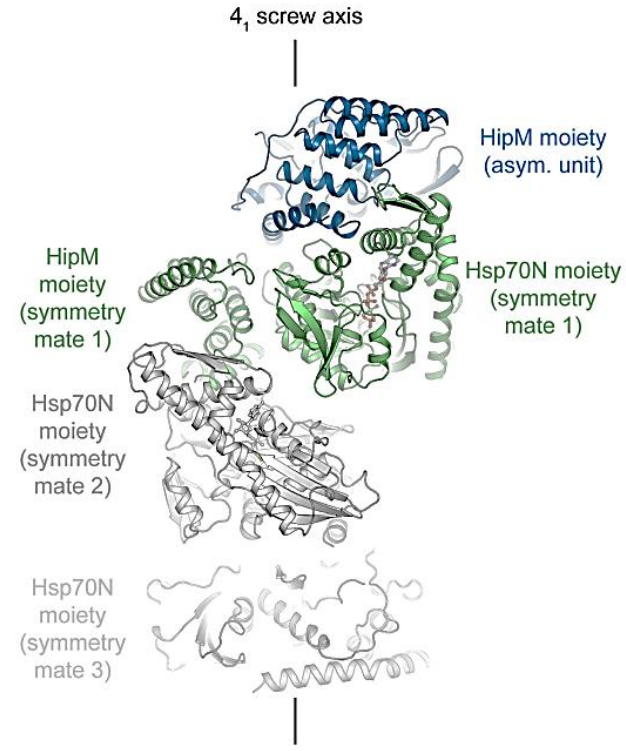

Figure 30: Intramolecular- and intermolecular interfaces of the Hsp70N-HipM fusion protein.

A) Crystal structure of the Hsp70N-HipM fusion protein showing the intramolecular domain-domain interface. The protein structure is shown in ribbon representation. The Hsp70N and HipM segments are colored green and dark blue, respectively; subdomain IIB of Hsp70N is highlighted in yellow. The bound ADP is shown in stick representation.

B) Intermolecular interface between HipM and Hsp70N with functional contacts.

C) Packing of Hsp70N-HipM fusion protein units in the crystal lattice. The functional contact between Hsp70N and HipM moieties from symmetry mates in the crystal lattice is high-lighted in blue (HipM partner) and green (Hsp70N partner). The fusion proteins pack via these functional HipM-Hsp70N contacts into suprahelical fibers along the $4_{1}$ vertical screw axis.

D) Lack of surface conservation of HipM at the intramolecular interface of the non-functional Hsp70N-HipM fusion protein.

E) Increased surface conservation of HipM at the intermolecular interface of functional Hsp70N-HipM fusion protein. 


\subsubsection{Intramolecular interface}

The fused Hsp70N and HipM domains were tightly associated in the crystal structure (Fig. 30A). The observed intramolecular domain-domain interface buried $949 \AA^{2}$ of surface area, potentially sufficient for a stable interaction. Hip is located on the side of subdomain I of Hsp70 and makes no contacts to subdomain IIB of Hsp70N, which is a well-known to regulate access to the nucleotide binding pocket (Liu et al., 2010). It is hard to see how this binding mode of Hip could stabilize Hsp70 in the ADP-bound state. The interaction of Hip with Hsp70 is on the convex face of the TPR domain instead of the concave face (Fig. 30D). Moreover, this interface proved to be poorly conserved, which is in conflict with surface sequence conservation normally found at protein-protein interfaces because of co-evolution of the binding partners. Of note, the surface of the NBD of Hsp70 is strongly conserved all over. Together this raises strong doubts on the functional relevance of the intramolecular interface.

Moreover, this interaction is apparently forced by favorable contacts to the artificial linker between Hsp70 and HipM, residues Leu76 and Glu77, which were introduced during cloning, the respective sequence serving as a restriction site between the Hsp70 and HipM coding sequences (Fig. 31).

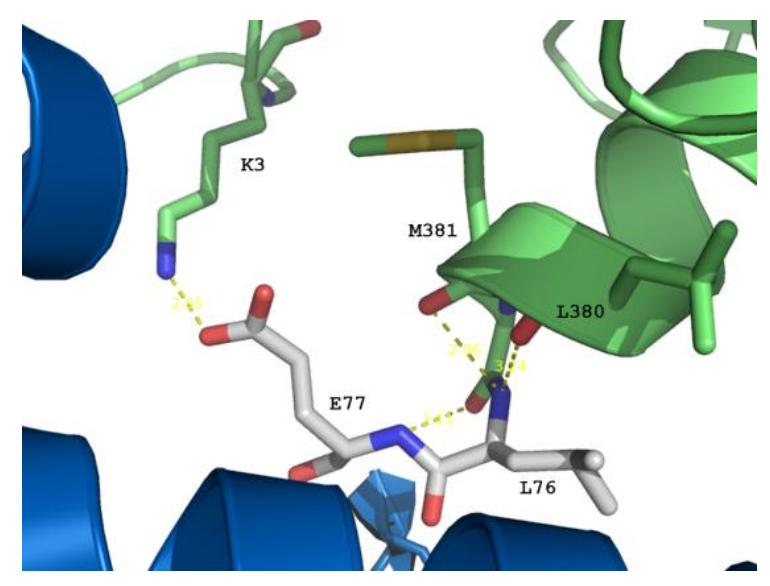

Figure 31: Detailed interactions between the artificial linker, Hsp70 and HipM.

The linker residues undergo multiple polar interactions. Back bone hydrogen bonds are formed between Leu76/Glu77 and Leu380/Met381 at the C-terminus of Hsp70N (color green), respectively. In addition, a likely salt bridge between Glu77 and side chain of Lys3 of Hsp70 was observed. 
Remarkably, the linker was structured, forming an extension to the C-terminal final helix of Hsp70N. To prove that the intramolecular interface is without function, the double mutation H196S/E199A targeting interruption of the intramolecular interface was investigated in our functional assays (see below). We confirmed that this mutation is indeed neutral, suggesting that the contact is an experimental artifact.

\subsubsection{Intermolecular interface}

Surprisingly, an intermolecular interface of Hsp70N-HipM fusion proteins forming a contact between symmetry mates in the crystal lattice showed the characteristics of a functional interface (Fig. 30B \& 30E). The interaction buries $1039 \AA^{2}$ surface area, similar to the intramolecular interface $\left(949 \AA^{2}\right.$ ). Moreover, the surface of HipM shows the highest conservations score between residue 176-181 and 210-214 (Fig. 32), which contact subdomains IA, IB and IIB of Hsp70N in the intermolecular interface (Fig. 30B \& Fig. 30E). This is in line with the regulatory role of subdomain IIB of Hsp70. The intermolecular contact therefore provides a promising candidate for the functionally relevant interface between Hip and Hsp70.

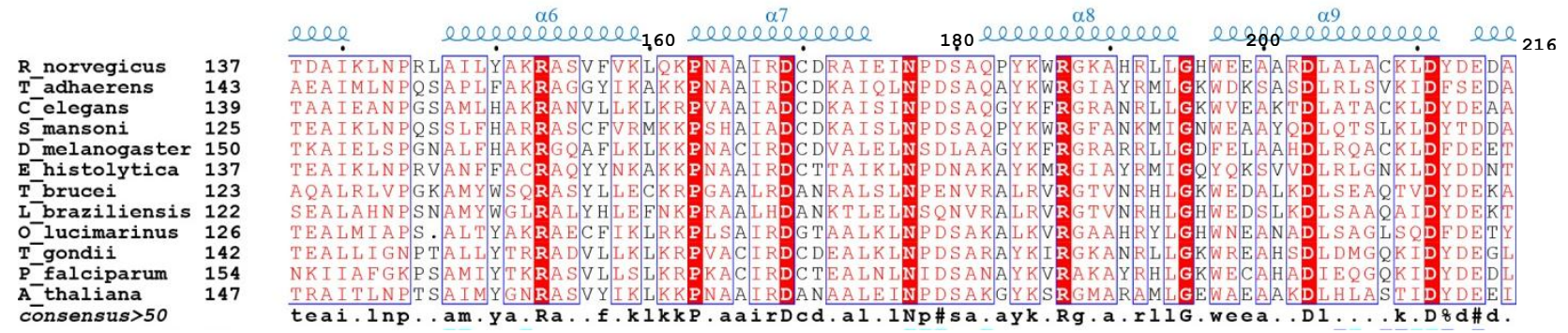

Figure 32: Evolutionary conservation of the central part of HipM, from residues 137 to 216.

In this section of the alignment of Hip sequences similar residues are shown in red and identical residues in white on red background, respectively.

\subsubsection{Structure of the Hip:Hsp70 core complex}

As mentioned above, the crystal lattice suggested a promising candidate for the interface between Hip and Hsp70. This interface and some key residue contacts are described in detail be- 
low (Fig. 33). We later confirmed the functional significance of this interface by mutational analysis (see below).

The NBD of Hsp70 is thought to be conformationally dynamic, with subdomain IIB acting as a mobile regulator for the nucleotide (Liu et al., 2010). The crystal structure of HipM:Hsp70 core complex showed that Hip forms a bracket over the NBD, arresting the otherwise mobile subdomain IIB in a closed conformation and locking the nucleotide in the binding pocket (Fig. $33 \mathrm{~A})$.

A)

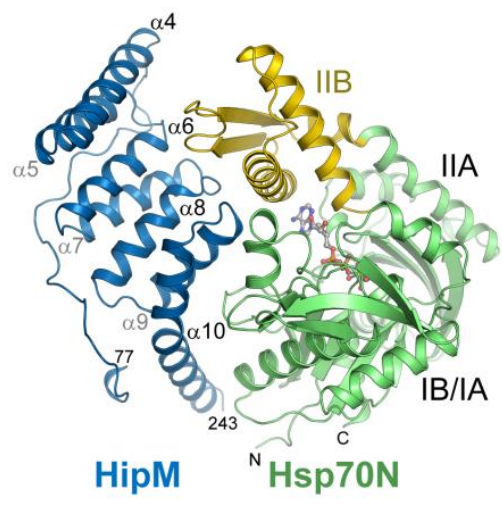

B)

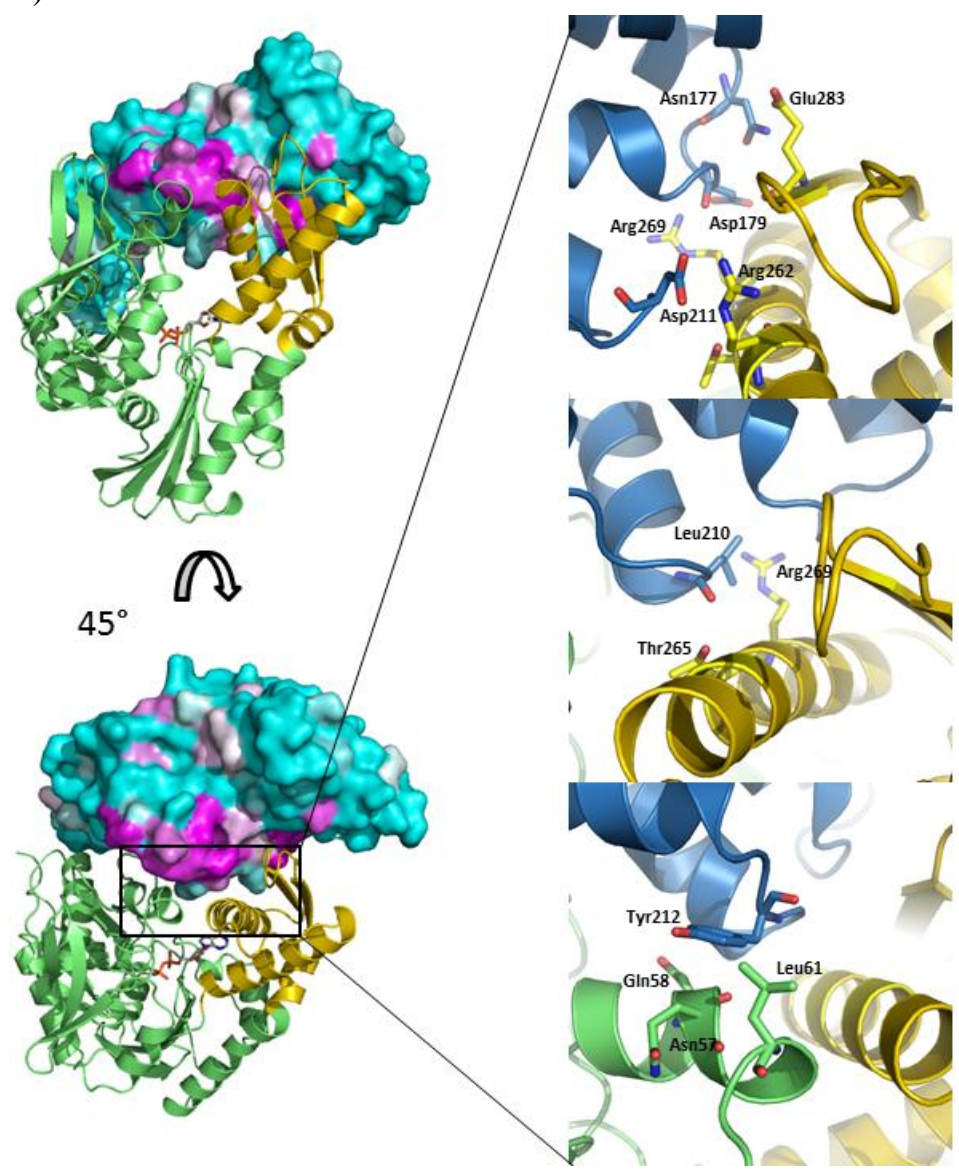

Figure 33: Structure of core complex of Hip and Hsp70.

A) Ribbon representation of the HipM:Hsp70 complex. HipM and Hsp70N are colored blue and green, respectively. Subdomain IIB of Hsp70N is highlighted in yellow. The bound ADP-phosphate- $\mathrm{Mg}^{2+}$ complex are shown in ball-and stick representation. The subdomain structure of the Hsp70N is indicated.

B) Left panel: Surface conservation of the interface between HipM and Hsp70N. The similarity score from thesequence alignment (Appendix 8.2) is mapped onto the molecular surface of HipM using a color gradient from magenta (high similarity) to cyan (low similarity). Right panel: Key contacts between HipM and Hsp70N are shown in detail. 
The conformation of $\mathrm{Hsp} 70 \mathrm{~N}$ in complex with HipM is virtually identical to that of the ADP.Mg.P $P_{i}$ complex of the NBD of bovine Hsc70 (Wilbanks and McKay, 1995), with the nucleotide being deeply buried in the center of the structure (Fig. 34A). Conversely, Hsp70 NEFs accelerate ADP release by stabilizing Hsp70N in an open conformation.

A)

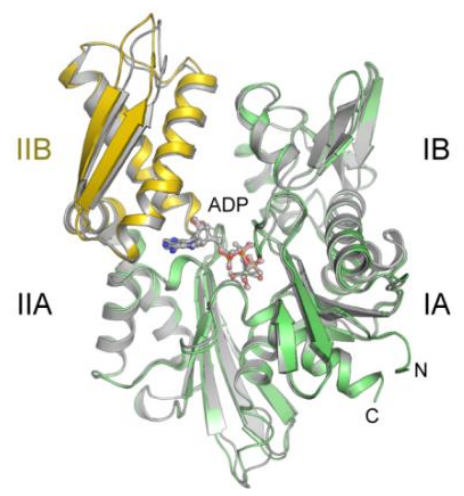

B)

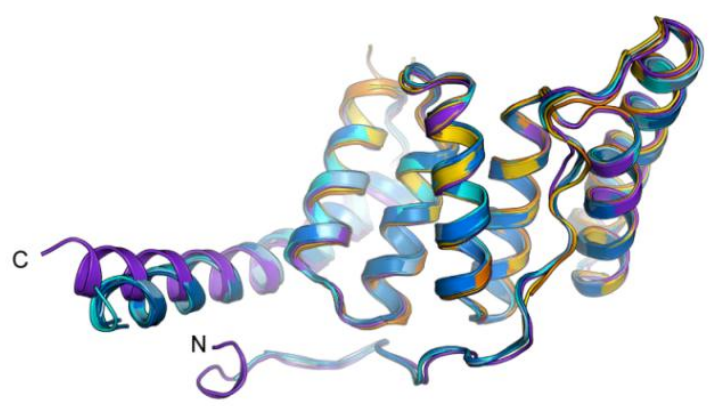

Figure 34: Analysis of Hip:Hsp70 interaction.

A) Superposition of Hsp70N from the Hsp70N-HipM structure with the ADP. $\mathrm{P}_{\mathrm{i}} \cdot \mathrm{Mg}^{2+}$ complex of the NBD from bovine Hsc70. The latter structure is shown in gray (PDB code 1HPM (Wilbanks and McKay, 1995)). The subdomain structure is indicated. The Hsp70N fragment has essentially the same structure with an r.m.s.d. of $0.674 \AA$, consistent with Hip recognizing and stabilizing the ADP-bound state.

B) Superposition of HipM structures. The copies of HipM in the asymmetric units of crystal forms I (two copies) and II (four copies) were superposed on the respective segment in the structure of the fusion protein. Backbone traces from crystal forms I and II are indicated by yellow and blue, respectively. The backbone of the Hip segment in the fusion protein is shown in purple. Both of the $\mathrm{N}$ - and $\mathrm{C}$-termini are indicated.

The structure of HipM in the Hip:Hsp70 complex is essentially identical to the structure of HipM alone in crystal form II (r.m.s.d.s $0.834-0.873 \AA$ ), indicating that the functionally active conformation is favored also in absence of Hsp70. Since the conformation of HipM in the complex with Hsp70N is virtually indistinguishable from that of the isolated domain, the surface of HipM for Hsp70 binding is probably pre-formed (Fig. 34B). The extended interface with Hsp70N at the upper edge of the Hip TPR domain is complementary in shape to the surroundings of the Hsp70 nucleotide binding cleft. This mode of interaction differs markedly from other TPR modules, most of which bind extended peptides in a groove at the concave face of the TPR solenoid (D'Andrea and Regan, 2003). 
The intermolecular interface comprises a large number of polar and hydrophobic interactions, shown in right panel of Figure 33B: (i) Asn177 of HipM forms a hydrogen bond with Glu283 in Hsp70N; (ii) Asp179 and Asp211 engage in salt bridges to Arg269 and Arg262 in Hsp70N, respectively; (iii) Tyr212 of HipM is in van-der-Waals contact to residues Asn57, Gln58 and Leu61 in lobe IB of Hsp70N; (iv) Leu210 forms hydrophobic contacts to residues Arg269 and Thr265 of Hsp70N. The interaction of the TPR core domain with subdomains IA, IB and IIB of Hsp70N is reinforced by contacts of the elongated terminal helix $\alpha 10$ of Hip with subdomain IA. This rather shallow interface appears to be stabilized by electrostatic interactions between numerous basic residues (233-HRRKYERKR-241) at the C-terminus of helix $\alpha 10$ and the helix dipole of helix $\alpha 4$ in Hsp70N.

\subsubsection{Mutational analysis of the binding interface between Hip and Hsp70}

Since a large number of residues within the conserved interface were involved in the interaction between Hip and Hsp70, we next probed the functional significance of the interface by mutation of key residues in Hip, using the MABA-ADP dissociation assay as a read-out.

The mutations were introduced in HipM, Hip residues 78-247 (Fig. 35B), because this fragment exhibited a similar effect on MABA-ADP dissociation as full-length Hip (Fig. 35A). The assay is a suitable proxy for the Hsp70 binding ability.

A)

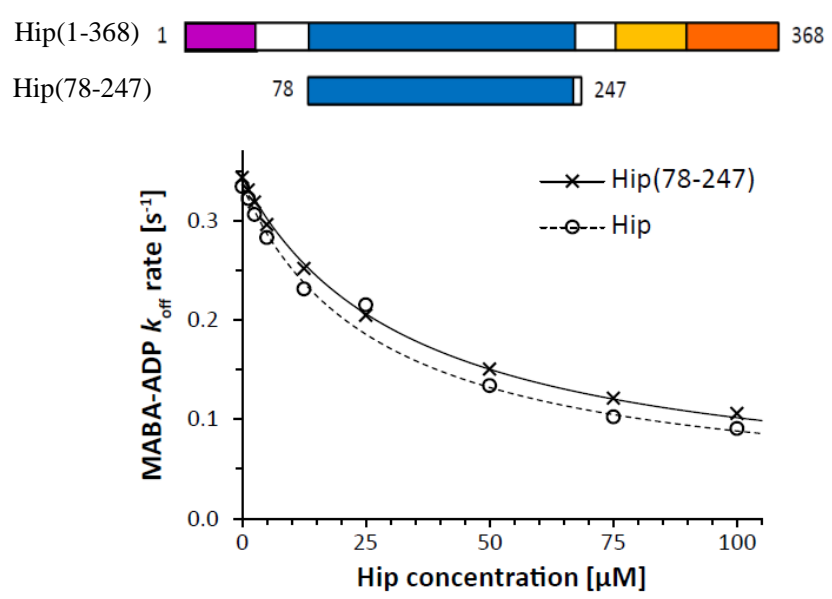

B)

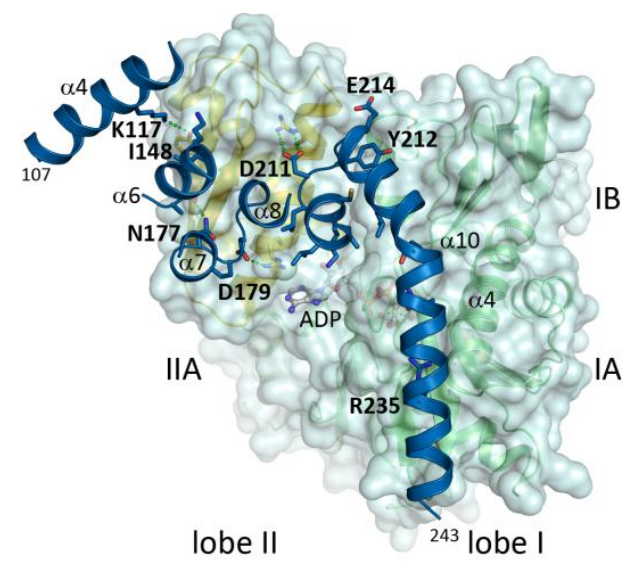


C)

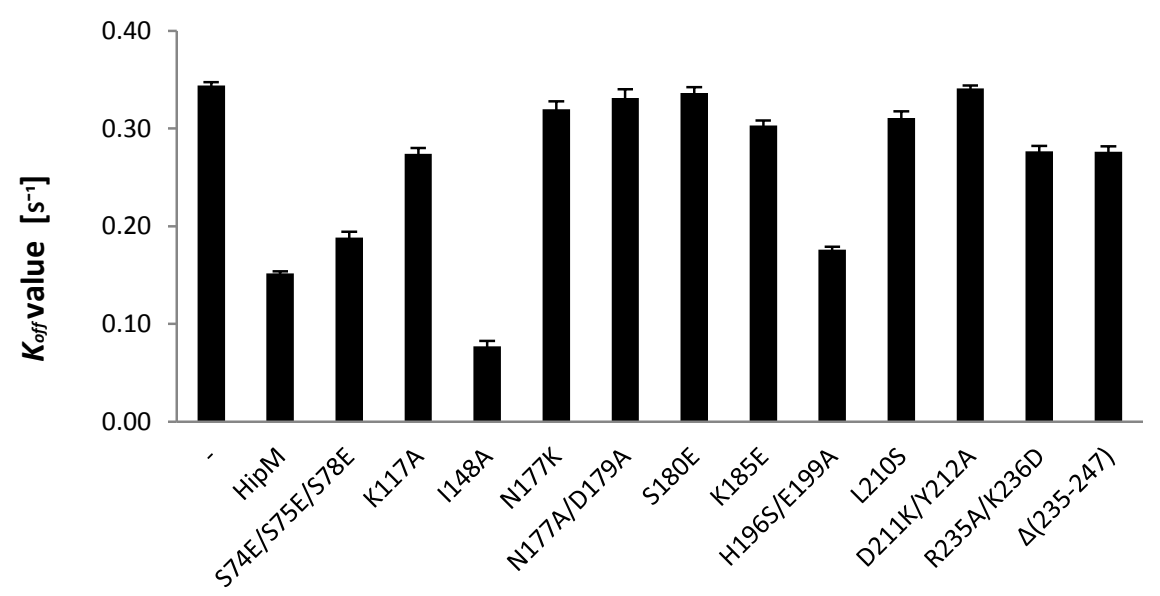

D)

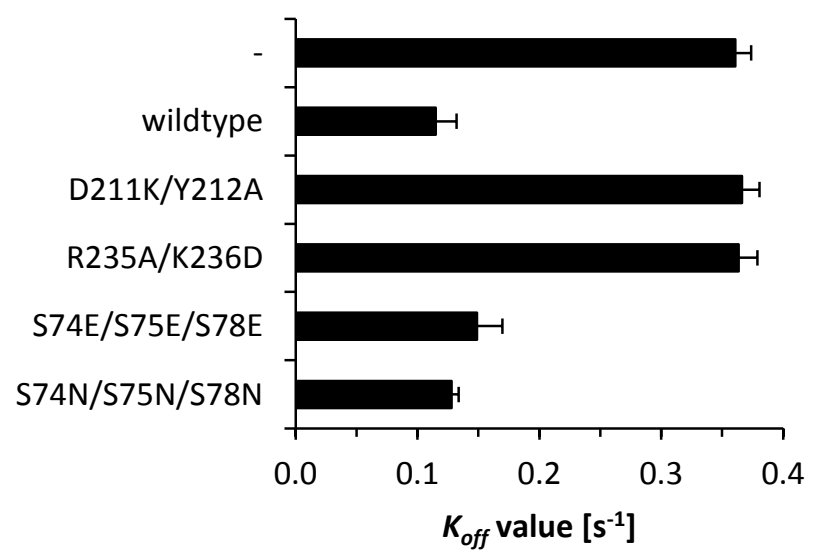

Figure 35: Mutational analysis of the interaction between Hip and Hsp70.

A) Schematic representation of the full-length Hip(1-368) and Hip(78-247) (HipM) and the analysis of MABAADP release assay. Hip-mediated attenuation of MABA-ADP dissociation from Hsp70N. In a stopped-flow apparatus, $2.5 \mu \mathrm{M} H \operatorname{Hsp} 70 \mathrm{~N}$ with MABA-ADP complex was mixed with $250 \mu \mathrm{M}$ ATP solution containing increasing concentrations of full-length Hip or HipM at $30^{\circ} \mathrm{C}$. Apparent $k_{\text {off }}$ rates were determined from the exponential decay of MABA-ADP fluorescence and plotted against the final Hip concentration.

B) Key contacts in the functional HipM:Hsp70N interface. The contacting elements of HipM are shown above the molecular surface of Hsp70N. Interacting side chains are highlighted in stick representation. Key polar contacts are indicated by green dotted lines. The bulk of the HipM TPR domain in the foreground was removed for clarity.

C) Probing the interface between Hip and Hsp70 by HipM mutation. Hsp70N•MABA-ADP was mixed with a 40 -fold excess of the respective HipM mutant. $K_{\text {off }}$ rates of MABA-ADP obtained from the resultant fluorescence traces are shown. Error bars represent standard deviations from at least three independent experiments.

D) Probing the interface of full-length Hip by mutation. Hsp70N•MABA-ADP was mixed with a 40-fold excess of the full-length Hip mutants. 
Mutations N177K, N177A/D179A, L210S and D211K/Y212A in the central region of the interface resulted in a virtual loss of the ability of Hip to decelerate the dissociation of MABAADP from Hsp70N (Fig. 35B \& 35C). The mutation N177K resulted in very weak binding to Hsp70-ADP, as determined by ITC (Table 15). The double mutant D211K/Y121A resulted in virtually complete loss of binding in ITC. The inactivating mutation K185E disrupts a conserved intramolecular salt bridge with Asp213, and thus presumably destabilizes Hip. Mutations K117A, R235A/K236D and $\Delta(235-247)$ at the periphery of the interface exhibited a similar milder functional impairment. The double mutation R235A/K236D at the C-terminus of HipM was intended to prevent dissipation of the dipole moment in $\alpha 4$ helix in Hsp70N by Hip. It induced a similar $K_{\text {off }}$ rate as the truncation construct of HipM, $\Delta(235-247)$, consistent with an important role of dissipation of the dipole moment for binding.

\begin{tabular}{cc}
\hline HipM mutation & $\mathbf{K}_{\mathbf{D}}$ value $[\boldsymbol{\mu M}]$ \\
\hline N177K & 192 \\
K185E & 85 \\
D211K/Y212A & n.d. \\
R235A/K236D & 68 \\
\hline
\end{tabular}

Table 15: The dissociation constants of selected HipM mutants determined by ITC. As expected, the mutations showed lower binding affinity to Hsp70N than wild type HipM $\left(K_{D}=27 \mu M\right)$, consistent with the MABA-ADP release assay.

Notably, mutation L210S (L211S in human Hip) was previously identified in a screen for lossof-function mutants of human Hip (Place, 2011). In contrast to the above listed mutants, the double mutation H196S/E199A targeting the non-conserved intramolecular interface of the Hsp70N-HipM fusion protein had no effect on HipM activity (Fig. 35C), a result confirming that this interface is not functionally relevant.

Subsequently, two phosphomimetic mutations, S74E/S75E/S78E and S180E, were tested. Cell cycle-dependent phosphorylation of Hip at S74, S75 and S78 was previously detected in a large scale proteomics studies (Olsen et al., 2006). Phosphorylation at Hip residue S180 was independently found (Olsen et al., 2010). We found that the phosphor-mimetic mutant S180E located at the interface was inactive (Fig. 35C). However, the triple mutation S74E/S75E/S78E had activity similar to wild type, which is most likely because of its location far from the Hsp70 
interface. Interestingly, the mutation I148A located within the functional Hsp70N-HipM interface was reported before to increase Hsp70 binding activity (Nelson et al., 2004). This surprising finding could be confirmed with our functional assay. The mutation might enhance polar contacts of Lys117 and Lys152 with Asp292, Tyr294 and Asp285 of Hsp70N, respectively. The finding that the I148A mutation has not been selected for during evolution suggests that in vivo the affinity of Hip for Hsp70 must be carefully balanced with that of other Hsp70 regulators.

To complete the analysis, we further tested selected mutations in the context of full-length Hip (Fig. 35D). The selected mutations all exhibited the same trend in the functional assay as the respective mutations in HipM. Only the double mutation R235A/K236D showed a more serious functional impairment in full-length Hip compared to the HipM mutant. No clear difference was observed between the phosphor-mimetic S74E/S75E/S78E mutation and the phosphorylation-resistant mutant S74N/S75N/S78N, showing that modification at this site can only indirectly affect Hip activity.

In order to test for subtle differences between mutations, which virtually abolish Hip function in the HipM construct, some of these mutations were introduced into the HipM-Hsp70N fusion protein with the functional intramolecular interface, which has been identified in section 4.4 (Fig. 21).

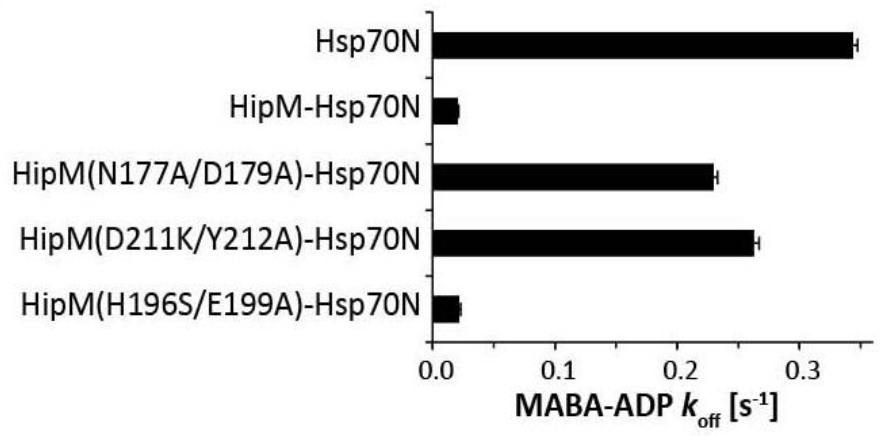

Figure 36: Point mutations in the intramolecular interface of the HipM-Hsp70N fusion protein. Mutants markedly accelerate MABA-ADP dissociation, whereas the mutation H196S/E199A in the HipMHsp70N interface is neutral.

The double mutation D211K/Y212A in HipM-Hsp70N almost restored the MABA-ADP offrate to the level of free Hsp70N (Fig. 36). The double mutant N177A/D179A had a somewhat 
milder effect. As expected, the double mutation H196S/E199A in the non-functional interface left the off-rate of HipM-Hsp70N unchanged (Fig. 36).

Taken together, the mutational analysis fully validated the intermolecular contacts in the Hsp70N-HipM crystal structure as the functional binding interface between Hip and Hsp70.

\subsection{Interplay between Hip and Hsp70 NEFs}

Two co-chaperones target the ADP state of Hsp70. Hip thereby stabilizes this state, whereas NEFs stimulate the release of ADP and the progression of the Hsp70 folding cycle. Therefore, we hypothesized that Hip and NEFs might act as direct competitors for Hsp70 binding.

To explore this idea, we mapped the binding areas of Hip and NEFs on Hsp70N based on the solved complex structures. Figure 37A shows that the binding surfaces on the Hsp70 NBD strongly overlap, suggesting mutually exclusive binding of Hip and NEFs to Hsp70 (Polier et al., 2008; Schuermann et al., 2008; Shomura et al., 2005; Sondermann et al., 2001; Xu et al., 2008).

A)

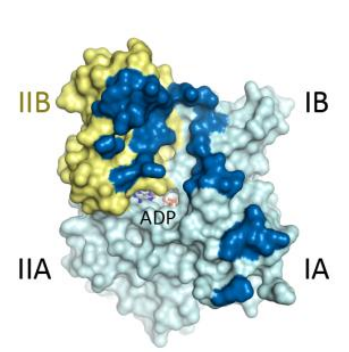

Hip

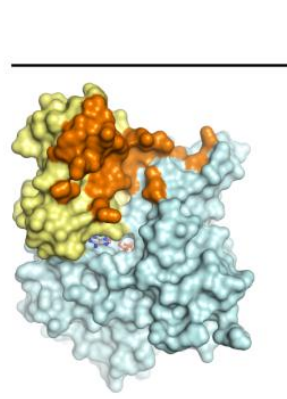

BAG1

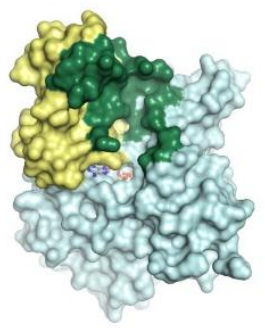

BAG2

NEFS

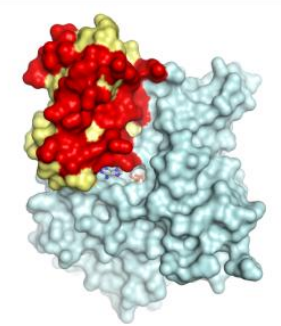

HspBP1

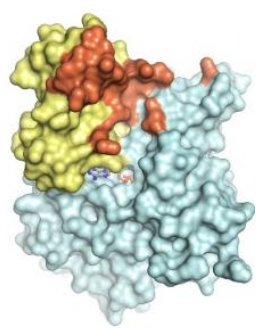

BAG5

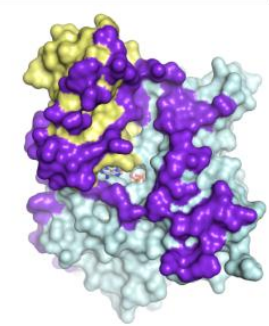

Sse1 (yeast Hsp110) 
B)

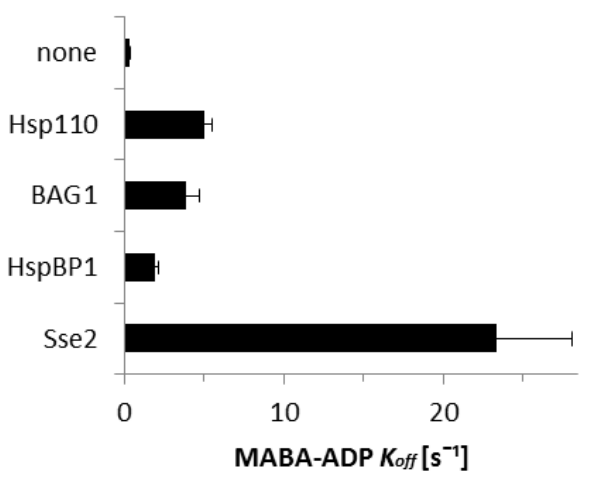

D)

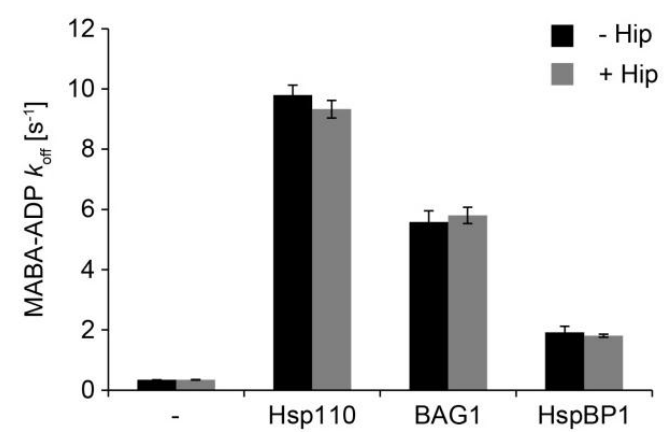

C)

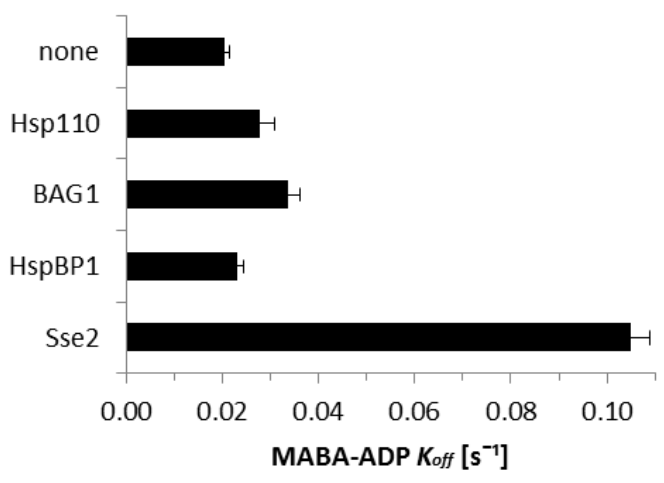

E)

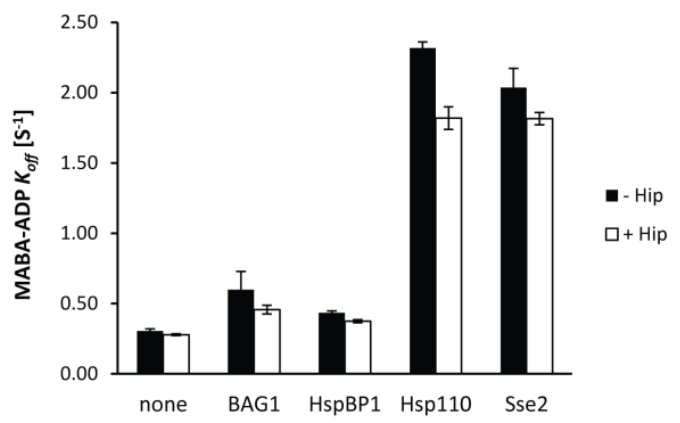

Figure 37: Interplay of Hip and Hsp70 NEFs.

A) Comparison of Hip- and NEF-binding surfaces on Hsp70N. Residues of the NBD that form contacts with Hip, BAG1, BAG2, BAG5, HspBP1 and Sse1 in the respective crystal structures are indicated on the surface of the human Hsp70NBD in the ADP state. Subdomain IIB of the NBD of Hsp70 is highlighted in yellow. In the crystal structures of NEF-Hsp70 NBD complexes, subdomains IIB are rotated outwards. The nucleotide binding pocket is located in the center of the molecule.

B) Effect of NEFs on MABA-ADP dissociation from the Hsp70N•MABA-ADP complex in the absence of Hip. Equimolar concentrations $(2.5 \mu \mathrm{M})$ of the Hsp70 and NEFs (Hsp110, BAG1, HspBP1 and Sse2) were used in the assay. Error bars represent standard deviations from three independent experiments.

C) Rates of MABA-ADP dissociation from the functional HipM-Hsp70N fusion protein in the presence of NEFs (Hsp110, BAG1, HspBP1 and Sse2). Equimolar concentrations of the proteins (2.5 $\mu \mathrm{M})$ were used. Please note the 100-fold difference in scale compared to panel B

D) Effect of Hip on MABA-ADP dissociation from Hsp70N in the presence of NEFs (Hsp110, BAG1, HspBP1). Equimolar amounts of the proteins were used $(2.5 \mu \mathrm{M})$. MABA-ADP $K_{\text {off }}$ rates for Hsp70N (black) and an equimolar mixture of Hip and Hsp70N (gray) are shown. Error bars represent standard deviations from three independent experiments.

E) Competition at likely cytosolic concentration ratios. The complex of MABA-ADP and Hsp70N (2.5 $\mu \mathrm{M})$ was mixed with buffer containing ATP $(250 \mu \mathrm{M})$ and NEFs (BAG1, HspBP1, Hsp110, Sse2) $(0.5 \mu \mathrm{M})$ either in presence or absence of Hip $(2.5 \mu \mathrm{M})$ at $30^{\circ} \mathrm{C}$ in a stopped-flow apparatus. $K_{\text {off }}$ rates for Hsp70N (black) and an equimolar mixture of Hip and Hsp70N (white) are shown. Error bars represent standard deviations from three independent experiments. 
First we tested the stimulatory effect of NEFs on the MABA-ADP dissociation from the Hsp70N•MABA-ADP complex in the absence of Hip. Equimolar amount of Hsp70 and NEFs (Hsp110, BAG1, HspBP1 and Sse2) were studied (Fig. 37B). Compared to spontaneous ADP dissociation from Hsp70 $\left(k_{o f f} \sim 0.34 \mathrm{~s}^{-1}\right)$, all the NEFs strongly accelerated ADP release. Addition of equimolar amounts of Hip had almost no effect (Fig. 37D), in line with the roughly 100-fold higher affinity of NEFs for Hsp70 found in previous studies (Raviol et al., 2006; Shomura et al., 2005; Sondermann et al., 2001). However, the maximal stabilization effect of Hip can be observed by employment of the fusion protein HipM-Hsp70N (Fig. 37C). Interestingly, the yeast Hsp110 protein Sse2 competed somewhat more efficiently, perhaps because a Hip homolog does not exist in yeast (Fig. 37B).

Hip was reported to be present in the cytosol at concentrations similar to Hsc70 ( $1 \mu \mathrm{M})$ (Höhfeld et al., 1995), while most NEFs are present only at 10-fold lower levels. To mimic a more physiological ratio in the MABA-ADP release assay, equimolar amounts of Hip and Hsp70 in 5-fold molar excess to NEFs were used (Fig. 37E). This excess was however not sufficient to suppress NEF action on Hsp70.Therefore, Hip cannot efficiently interfere with the binding of NEFs to Hsp70N under the assay conditions.

Since the free Hip cannot efficiently compete with the binding of NEFs under the assay conditions, we employed the functional fusion protein HipM-Hsp70N as a model for maximum Hip binding (Fig. 38).

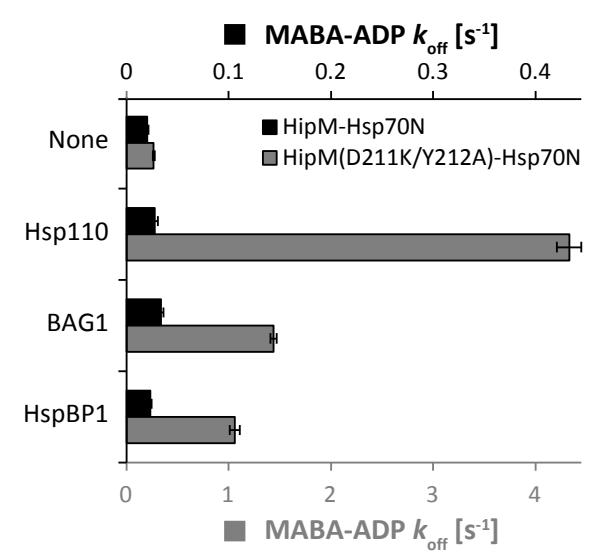

Figure 38: Effect of mutations in the HipM-Hsp70N interface on NEF-triggered MABA-ADP release. Rates of MABA-ADP dissociation from HipM-Hsp70N fusion proteins in the presence of NEFs (Hsp110, BAG1, HspBP1). Equimolar concentrations of the proteins were used. $K_{\text {off }}$ rates for the functional HipM-Hsp70N fusion protein (black) and the inactive mutant HipM(D211K/Y212A)-Hsp70 (gray) are shown with standard deviations from three independent measurements. Note the difference in scale of the $\mathrm{X}$-axes. 
Virtually no acceleration of MABA-ADP release was observed, when stoichiometric amounts of the mammalian NEFs (Hsp110, BAG and HspBP1) were added to the MABA-ADP complex of the functional HipM-Hsp70N fusion protein (black bar in Fig. 38). The $k_{\text {off }}$ rate of the MABA-ADP dissociation remained below $\sim 0.04 \mathrm{~s}^{-1}$. The NEF effect was restored in the double mutation HipM(D211K/Y212A)-Hsp70N, which disrupts the functional interface between Hip and Hsp70, reflecting that presence of HipM attached to Hsp70N (gray bar in Fig. 38).

In the presence of saturating concentrations of Hip, the NEFs thus do not effectively bind to Hsp70. When Hip binding is reinforced by other means, Hip should therefore effectively compete with NEFs.

\subsection{Hip function in vivo}

To evaluate the functional relevance of the dimerization and of the putative substrate binding region of Hip in vivo, we employed a $\beta$-galactosidase reporter (GR). This established in vivo model system evaluates the effects of various molecular chaperones on the hormone-dependent maturation of glucocorticoid receptor (GR) in S. cerevisiae. (Nelson et al., 2004). In this model system lacking endogenous Hip, GR activity is measured via the expression of a steroiddependent $\beta$-galactosidase reporter (Fig. 39A). Almost complete conservation of the Hip contact residues in the yeast Hsp70s Ssa1 and Ssa2 suggests that mammalian Hip interacts functionally with yeast Hsp70. At limiting hormone levels, Hip expression increased the reporter activity $\sim 3$-fold, reflecting the ability of Hip to enhance the conformational maturation of GR (Fig. 39C)

A)

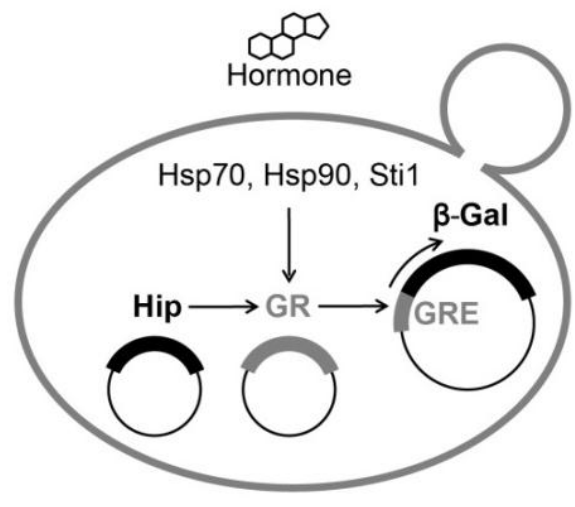


B)

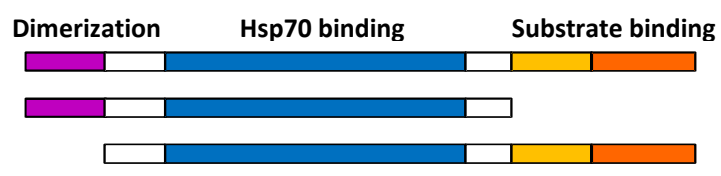

C)

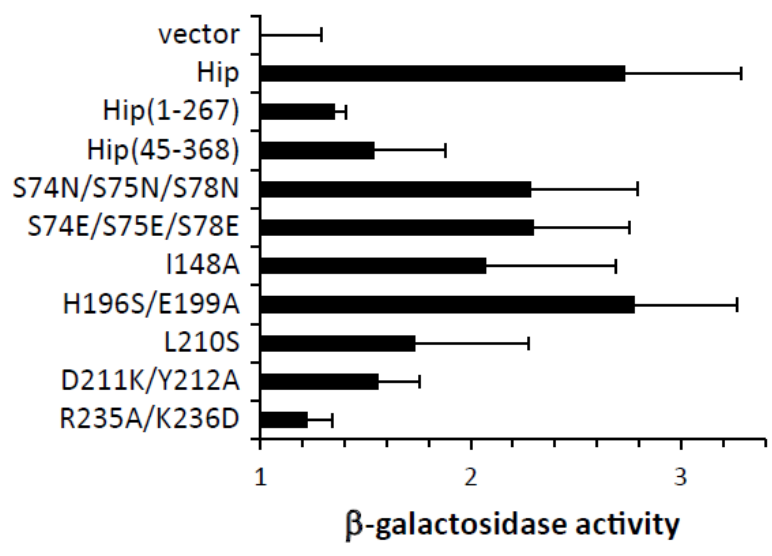

F)

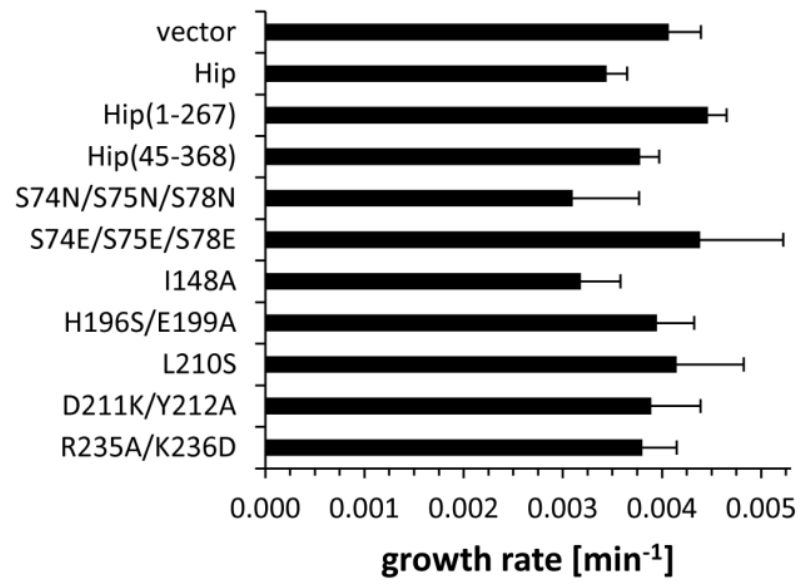

Figure 39: Hip functional study in vivo.

A) The model system. Hip constructs and the glucocorticoid receptor (GR) protein from R. norvegicus were coexpressed in a $S$. cerevisiae reporter strain expressing $\beta$-galactosidase ( $\beta$-Gal) under control of the hormonedependent GR promoter; GRE (Riggs et al., 2003). Hip and GR functionally interact with the yeast Hsp70 and Hsp90 machinery (Hsp70, Hsp90, Sti1).

B) Schematic representation of the Hip, Hip(1-267) and Hip(45-368) constructs.

C) Reporter gene activity upon expression of Hip mutants. $\beta$-Gal levels were measured upon the expression of Hip constructs. The relative amounts of functional GR compared to control cells without expressing Hip are indicated. Averages and standard deviations (s.d.) are from five independent experiments. 
D) Expression levels of Hip variants in yeast cells. Western blot of yeast reporter strains. S. cerevisiae cultures were grown to OD 1.5-2.0 at $30{ }^{\circ} \mathrm{C}$ in selective medium. Equal amounts of cells were harvested and subjected to alkaline lysis. Cell extracts were separated on $12 \%$ SDS-PAGE and probed by Western blotting against Hip using a polyclonal antiserum (Höhfeld et al., 1995). The strain transformed with p423ADH (empty vector) served as a control for background; phosphoglycerate kinase (PGK) was used as a loading control.

E) Western blot signal of purified Hip and Hip(45-368). Left panel: Equimolar amounts of purified Hip and Hip(45-368) were analyzed by Ponceau-S protein stain; Right panel: Western blotting with anti-Hip antibody.

F) The growth rate of the reporter strains. The growth rate for the different Hip variants in liquid shaking culture was analyzed by $\mathrm{OD}_{600}$ measurements during the assay.

To dissect the impact of different features of Hip on its in vivo functionality, we also expressed selected Hip mutants in the model system. All Hip variants had similar expression levels (Fig. 39D). The growth rates of the reporter strains were closely similar (Fig. 39F). In accordance with previous findings (Nelson et al., 2004), the monomeric construct Hip(45-368) had significantly reduced activity (Fig. 39B \& 39C), although the protein was expressed similar to wildtype Hip (Fig. 39D). This finding strongly suggests that avidity plays an important role in the interaction of Hip with Hsp70-substrate complexes. In addition, direct contacts of the substrate with the two arms of Hip might be important. This notion is supported by the poor in vivo activity of Hip(1-267), lacking the putative substrate binding region. However, in our in vitro MABA-ADP release assay, Hip(1-267) was as active as full-length Hip (Fig. 20E), suggesting that the direct interaction with Hsp70 is unperturbed in this mutant.

The mutants L210S, D211K/Y212A and R235A/K236D, which disrupted the Hip-Hsp70 interface similarly, diminished the ability of Hip to support GR activation. Thus, in vivo function of Hip depends on direct interactions with Hsc70 (Fig. 39C). Interestingly, expression of the hyper-active mutant I148A slightly reduced GR maturation relative to wild-type Hip. This is again evidence for careful control of Hip activity in vivo. Expression of Hip under a stronger promotor caused a temperature-sensitive phenotype in the reporter system (Nelson et al., 2004). The phosphomimetic S74E/S75E/S78E mutant and the S74N/S75N/S78N mutation used as negative control for in vivo phosphorylation were again more-or-less neutral (Fig. 39C), similar to the MABA-ADP release assay (Fig. 35D).

In summary, the in vivo studies of Hip suggested that function requires Hip dimerization and substrate binding capacity in addition to the capability to directly interact with Hsc70. 


\section{Discussion}

Protein folding to a well-defined conformation is essential to perform biological function. Moreover, misfolded proteins cause neurodegenerative diseases such as Alzheimer's, Parkinson's and Huntington's disease. The Hsp70 chaperones are central to the cellular defense against toxic protein aggregation through an ATP-dependent reaction cycle, which is regulated by co-chaperones of the J-protein family and by nucleotide exchange factors (NEFs).

Hip, Hsp70 interacting protein, is so far the only known Hsp70 co-chaperone that stabilizes Hsp70-client protein complexes by retarding the dissociation of ADP from Hsp70. In this study, we present the first complete structural model of this novel regulator of metazoan Hsp70 including the crystal structures of the dimerization domain and the TPR domain. We performed an in-depth mechanistic analysis of the interaction of the Hip core domain with the nucleotide binding domain (NBD) of human Hsp70. The structure-guided mutational analysis showed that Hip forms a bracket over the ATPase domain, reducing its otherwise dynamic nature and locking the ADP-bound state. Moreover, Hip directly counteracts the binding of various NEFs, which catalyze ADP dissociation and facilitate substrate release upon ATP re-binding. To become fully functionally effective, Hip must interact not only with the NBD but also with the Hsp70-bound client protein. Our data explains how Hip enhances aggregation prevention by Hsp70 and suggests how Hip would potentially divert the client proteins to the downstream proteostasis machinery, such as Hsp90 or the proteasome.

In the following sections, we contrast and compare the results of this study with previous studies and present an integrated model for the Hsp70 mechanism.

\subsection{Comparison of the TPR domains in Hip and Hop}

The basic function of TPR domains is to mediate protein-protein interactions, particularly facilitating interactions with heat shock proteins. The binding residues are normally presented on the concave face of the TPR solenoid and bind with high specificity to the target peptide (D'Andrea and Regan, 2003). Hop is the paradigm for TPR domain interaction with Hsp70 and Hsp90. The crystal structures of the TPR domain-peptide complexes revealed that Hop specifi- 
cally recognizes the C-terminal pentapeptides of Hsp70 and Hsp90 through distinct TPR domain modules (Scheufler et al., 2000).

A)

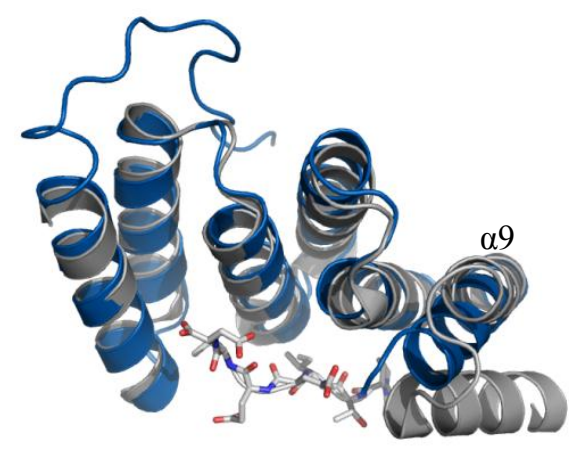

B)
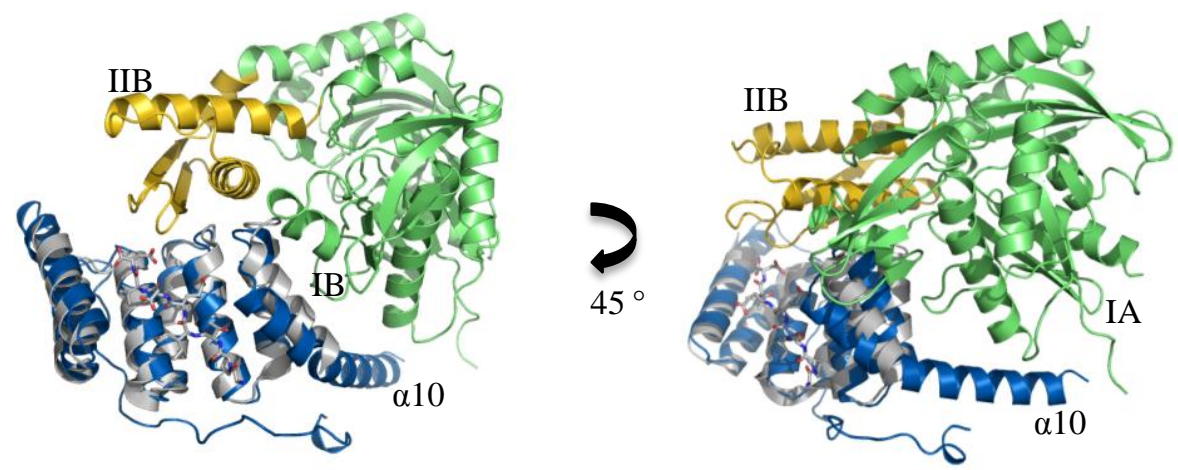

Figure 40: Comparison of the binding sites in the TPR domains of Hip and Hop.

A) Overlay of crystal form I of Hip(78-247) (dark blue) and the Hop-TPR1 peptide complex (gray). The proteins are shown in ribbon representation. The backbone of the Hop-bound peptide is represented as stick model. The last $\alpha$-helix of Hop is absent in form I of Hip(78-247).

B) Ribbon representation of the overlay between the complex structures of the Hip-Hsp70 and Hop-peptide. Hsp70N is shown in green with subdomain IIB in gold. Hip(78-247) is shown in blue.

In comparison to the TPR1 domain of Hop, the solenoid formed by the TPR repeats in Hip has a more pronounced curvature. However, unlike Hop, Hip does not employ the concave face of the solenoid for binding of Hsp70. It instead employs the upper edge of the solenoid, which is shape-complementary to the Hsp70 surfaces around the closed nucleotide binding cleft in subdomains IB and IIB (left panel in Fig. 40B). In addition, the elongated C-terminal helix $\alpha 10$ of Hip contacts lobe IA in the NBD of Hsp70 (right panel in Fig. 40B). This rather flat interface appears to be stabilized by electrostatic interactions with the negative end of one $\alpha$-helix dipole in Hsp70. Substitution of the positively charged residues Arg235 and Lys236 had a surprisingly 
strong effect on Hip function (see Table 15, Fig. 35C, Fig. 35D and Fig. 39C). The crystal structures of the middle domain of Hip show that the TPR domain is a versatile structural scaffold for protein-protein interactions that can support both interactions with extended peptide motifs as well as surface motifs in folded proteins.

\subsection{Tentative model for the overall structure of Hip dimer}

With structural models of the folded domains at hand, we can now construct a tentative model for the complete Hip dimer (Fig. 41).

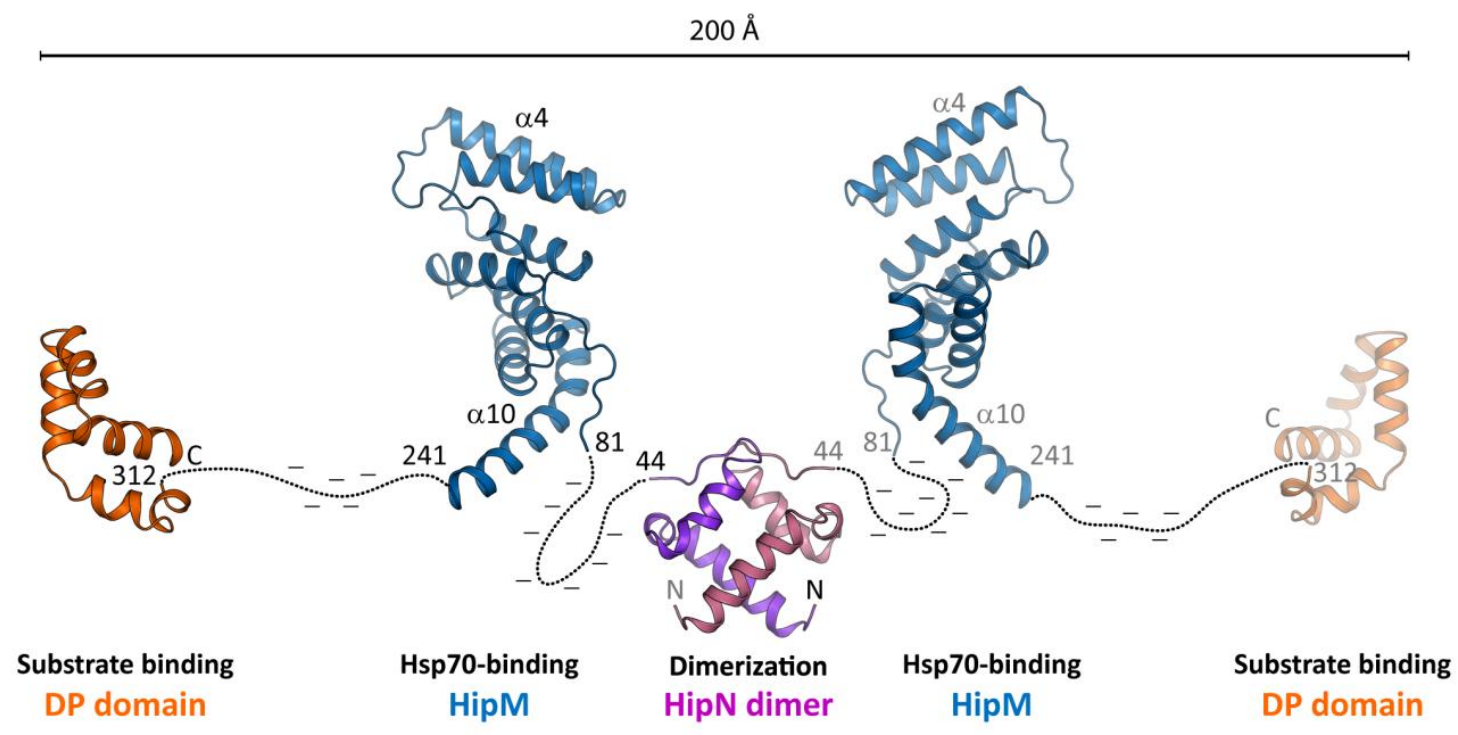

Figure 41: Tentative model of dimeric full-length Hip.

Consistent with previous observations (Dores-Silva et al., 2012; Velten et al., 2000), our small angle X-ray scattering (SAXS) analysis indicated an elongated conformation for the complete $\sim 80 \mathrm{kDa}$ Hip dimer with a maximum extension of $\sim 200 \AA$. In the model, it is important to note that all the folded domain structures are connected by long, negatively-charged linkers, which could span much greater distances (assuming a contour length of $3.6 \AA$ per residue). Considering the elongated shape of HipM, the DP domains at the ends of long tethers might not be resolved in the SAXS experiments at all, because of limited contrast against the solvent background. Electrostatic repulsion between the pronounced negative charges in the linkers might 
force the Hip dimer into an open, extended conformation, allowing independent and simultaneous binding of two Hsp70 molecules at each arm of the dimer.

Analysis of the CD spectra and the Kratky plot suggests the absence of regular secondary structure and disorder for most of the linker parts in Hip. This disorder may provide the conformational flexibility for Hip to recognize a wide range of Hsp70-substrate protein complexes.

The helices of the dynamic DP domains form hydrophobic grooves, which could serve as binding sites for exposed hydrophobic segments in non-native substrate proteins. Since the DP domains are expendable for Hsp70 binding in vitro, but important for the enhancement of the conformational maturation of GR in vivo, such interactions appear to play an important role for Hip functionality. This appears to work however only in dimeric Hip, suggesting an avidityenhanced binding mechanism requiring multiple recognition sites in the substrate complex.

\subsection{Specific recognition of the Hsp70 ADP state by Hip}

Consistent with the earlier proposal by Höhfeld et al. in 1995, our detailed ITC analysis demonstrated preferential binding of Hip to ADP-bound Hsp70 $\left(\mathrm{K}_{\mathrm{D}}=8 \mu \mathrm{M}\right)$, compared to the apo- and ATP states of Hsp70 $\left(\mathrm{K}_{\mathrm{D}}=51 \mu \mathrm{M}\right.$ and not determinable, respectively). Our analysis showed that the presence of ATP in the binding pocket of Hsp70 as such had only a minor influence.

Superposition of the Hip-Hsp70 core complex with the recently solved crystal structure of a mutant full-length bacterial Hsp70 (DnaK) in complex with ATP indicates how Hip might discern the ADP-bound from the ATP-bound form of the molecular chaperone (Kityk et al., 2012) (Fig. 42). 


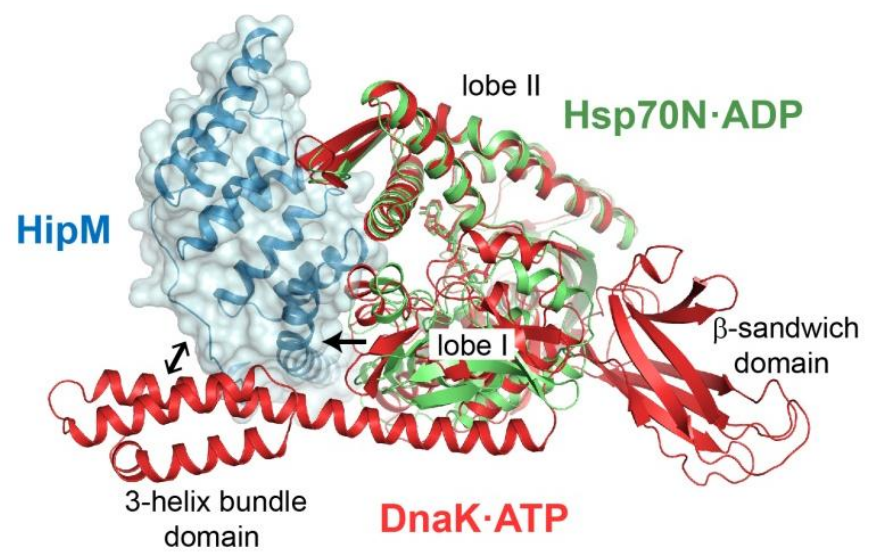

Figure 42: Comparison of the crystal structures of DnaK-ATP and the HipM-Hsp70N-ADP complex. An overlay of the two structures is shown. For superposition of the structures, the lobe II parts (subdomains IIA and IIB) of the NBDs were aligned. DnaK-ATP is shown in red, Hsp70N-ADP in green and HipM in blue ribbons, respectively. HipM is enveloped by a molecular surface.

In the ATP bound state, DnaK is in a compact conformation and the 3-helix bundle domain of the Hsp70 SBD is associated with the flank of the NBD. ATP binding to Hsp70 furthermore induces a twist between lobes I and II of the Hsp70 NBD, causing a slight rotation of lobe I of DnaK·ATP around the vertical axis, resulting in a large (4 $\AA$ ) relative shift of the top-left part of lobe I compared to ADP-bound Hsp70. This part contributes critically to the interface with Hip (indicated by an arrow). The ATP-induced shift would cause a severe clash with HipM. Therefore, Hip can no longer bind to both lobes of Hsp70 in the ATP-bound conformation simultaneously. Furthermore, the 3-helix bundle domain of the Hsp70 SBD would clash with the N-terminal region of HipM, which is indicated by a double arrow (Fig. 42). In the ADP-bound state, the 3-helix bundle domain of DnaK forms a lid over the peptide binding $\beta$-sandwich domain, which is loosely tethered via a flexible linker roughly opposite from the Hip interaction site. Thus it is unlikely to interfere with Hip binding.

The nucleotide-free state of Hsp70 (apo-state) is probably lowly populated in the nucleotiderich environment of the cytosol. In principle, it should have protein substrate affinity similar to the ADP-bound state, and as such should be a target for Hsp70-substrate complex stabilization. The lower affinity of Hip to this state is probably caused by increased flexibility of the Hsp70 NBD, as suggested by molecular dynamic simulations (Zhuravleva and Gierasch, 2011). This would transiently disrupt the simultaneous contacts of Hip to subdomains IB and IIB in Hsp70. 


\subsection{Mechanism of Hip-mediated stabilization of Hsp70 in the ADP state}

The crystal structure of the Hip:Hsp70 core complex revealed the molecular details of the functional interaction. Through a large number of polar and hydrophobic interactions, Hip stabilizes Hsp70N by forming a bracket over the nucleotide binding cleft, locking ADP in the nucleotide pocket. The binding interface involves the highly conserved edge of the TPR domain (residue 176-181 and 210-214) in Hip, which contacts subdomains IA, IB and IIB of Hsp70N.

A)

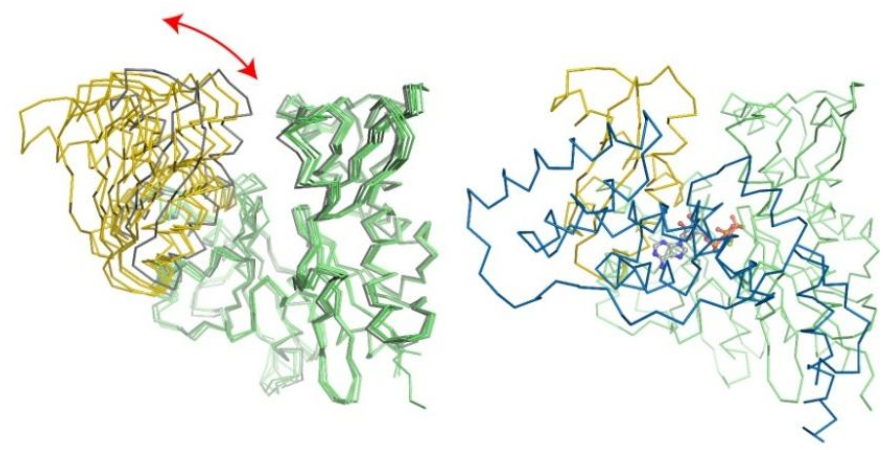

B)

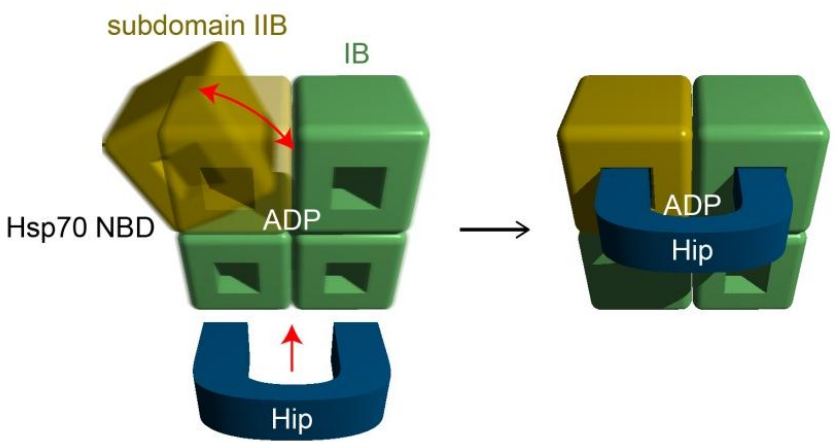

Figure 43: Interpretation of the Hip:Hsp70 structure.

A) Left panel: Superposition of Hsp70 NBD structures. Subdomain IIB indicated in yellow is attached via a flexible hinge to the rest of the NBD (green). This becomes apparent by comparing the conformations of the NBD in crystal structures of NBD:NEF complexes and the free ADP-bound form (gray) (Arakawa et al., 2010; Polier et al., 2008; Schuermann et al., 2008; Sondermann et al., 2001; Wilbanks and McKay, 1995; Xu et al., 2008). Right panel: The structure of the Hip:Hsp70 core complex is shown. Peptide backbones are represented as $\alpha$-carbon traces.

B) Schematic representation of structural dynamics of the Hsp70 NBD and the mechanism for stabilization of the closed conformation by Hip. The outward rotation of subdomain IIB - indicated by a curved red arrow breaks the respective contacts to ADP, thereby accelerating nucleotide dissociation (Liu et al., 2010). The contacts of the middle domain of Hip (dark blue) to subdomains IIB and IB/IA lock the domain in the closed conformation with ADP buried in the center. Absence of nucleotide greatly enhances the dynamics of the Hsp70 NBD, which would prevent stable Hip binding. In the ATP-bound conformation, the halves of the NBD are twisted with respect to each other, displacing the Hip-binding interface segments. 
Figure 44 shows an overview of the structural dynamics of the NBD of Hsp70. It is interesting to note the motion of subdomain IIB to allow ADP release, which is otherwise firmly surrounded by Hsp70 and cannot easily escape. A simulation of the rotatory movement is shown in Figure 44A by comparing the NBD in crystal structures of NBD:NEF complexes and the ADPbound form, which is closely similar to the structure of the NBD in the core complex with Hip. Interaction of Hip with both subdomain IIB and the opposing lobe I dampens this dynamic movement, thereby slowing dissociation of ADP.

\subsection{Antagonism between Hip and NEFs}

In principle, Hip and NEFs have opposing effects on the reaction cycle of Hsp70. Hip binding would rather slow or inhibit the cycle, while NEFs together with J-domain proteins would drive it forward. Comparison of the respective structures and our biochemical data show that Hip and NEF binding to Hsp70 are mutually exclusive.

Notably, Hip has much lower binding affinity to Hsp70 in vitro $\left(\mathrm{K}_{\mathrm{D}} \sim 10 \mu \mathrm{M}\right)$ than the NEFs, which have remarkably similar affinities $\left(\mathrm{K}_{\mathrm{D}} \sim 0.1 \mu \mathrm{M}\right)$ (Raviol et al., 2006; Shomura et al., 2005; Sondermann et al., 2001). The interaction between Hip and substrate-free Hsp70 appears to be largely, if not exclusively, mediated by the TPR domain of Hip (HipM) and the Hsp70 NBD, but with an affinity probably too low to be biologically relevant. In the cytosol, this may be partially compensated by a favorable concentration ratio between Hip and NEF (NEFs inhibit Hsp70 at 1:1 ratio). While the competition under physiological conditions remains to be experimentally investigated, our preliminary analysis of Hip-mediated attenuation of MABAADP dissociation from Hsp70N in the presence of $10 \mathrm{mM}$ phosphate suggests that increased concentrations of this metabolite might be in favor of Hip binding (Fig. 20C). In presence of phosphate, ADP appears to bind more tightly to Hsp70 (Arakawa et al., 2011), suggesting that the dynamics of the NBD is decreased. The binding characteristics of Hip as judged from the concentration dependence in presence of $10 \mathrm{mM}$ phosphate, appear unaltered (Fig. 20C). NEFs however appear to merely capture and stabilize the open conformation of the NBD, which will be less populated under such circumstances. Stabilization of ADP complexes of eukaryotic Hsp70 by phosphate might also explain why NEFs are required in the eukaryotic cytosol, alt- 
hough the spontaneous off-rate is sufficient to drive the folding reaction in vitro. This is in contrast to the bacterial homolog DnaK, which also depends on the NEF GrpE in vitro. The finding that the hyper-activating mutation I148A of Hip has not been selected during evolution indicates that Hip can effectively compete with NEFs under physiological conditions.

Under non-stressed conditions, Hip likely needs direct or indirect interactions with Hsp70bound substrate to effectively compete with NEFs. We were unable to demonstrate this effect in vitro with denatured firefly luciferase or carboxymethylated bovine $\alpha$-lactalbumin using our MABA-ADP release assay (data not shown). As judged from GR maturation, the putative substrate binding capability of Hip is as important as its capacity to interact with Hsp70 in vivo. However, avidity could greatly enhance the binding of Hip dimers to both substrates and Hsp70, as long as they form complexes with multiple binding sites.

Our findings therefore suggest that in order to effectively compete with NEF binding to Hsp70, Hip dimers would have to interact simultaneously with two Hsp70 molecules bound to different regions of the same substrate protein or to Hsp70s associated with small protein aggregates. The binding of multiple Hsp70 molecules may reflect the requirement of a specific substrate protein for prolonged chaperone stabilization. Thus, conformational properties of the client protein would determine the extent to which Hip decelerates Hsp70 cycling. Future experiments will have to test this element of the model, and the 140-residue protein $\alpha$-synuclein might be a promising substrate candidate, which was shown to undergo nucleotide-dependent interactions with Hsp70 (Roodveldt et al., 2009).

\subsection{Possible role of Hip in protein quality control}

Hip is so far the only known Hsp70 co-chaperone that stabilizes Hsp70-client protein complexes by retarding the dissociation of ADP from Hsp70. Previous model suggested the regulation of Hip as a tetramer to stabilize Hsp70 in the ADP-bound state (Ziegelhoffer et al., 1996).

Hsp70 is both involved in the folding of newly made and stress-denatured proteins as well as in the timely degradation of terminally misfolded proteins. Figure 44 shows an overview for the potential role of Hip in the cellular protein homeostasis machinery. 


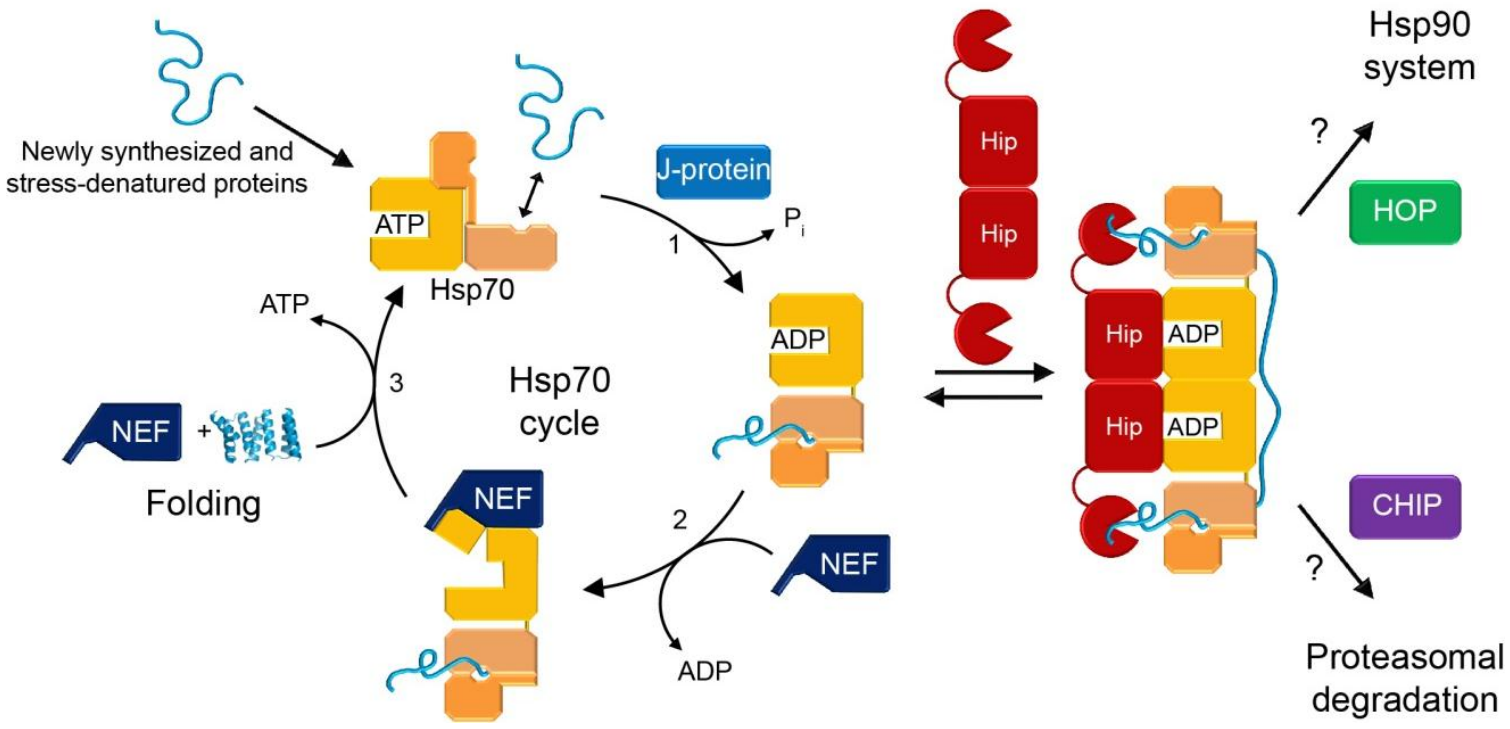

Figure 44: Model for the role of Hip in protein quality control.

A schematic model for the cooperation of Hsp70 and its cochaperones in protein folding is depicted. Hsp70 and Hip are indicated in yellow and red color, respectively. J-domain proteins and NEFs (blue color) drive the Hsp70 cycle of substrate binding and release. Hip attenuates Hsp70 cycling by stabilizing the ADP state, thereby increasing the substrate "holdase" activity of Hsp70 and diverting certain Hsp70-ADP-substrate complexes towards Hsp90 or proteasomal degradation.

J-domain proteins and NEFs drive the Hsp70 cycle of substrate binding and release. For both factors multiple isoforms co-exist in the cytosol, presumably adapting Hsp70 cycling rates to specific substrates.

Hip attenuates Hsp70 cycling by stabilizing its ADP state, thereby increasing the substrate "holdase" activity of Hsp70. This might occur by specific recognition of the bound substrate, enabling slow cycling and limiting unnecessary ATP expenditure on recalcitrant substrates. In addition, Hip preferentially targets and stabilizes Hsp70·ADP-substrate complexes containing multiple Hsp70 molecules. Binding of multiple Hsp70 molecules may reflect the requirement of a specific client protein for prolonged chaperone stabilization. Notably, the conformational properties of the client protein would determine the extent to which Hip decelerates Hsp70 cycling. Prolonged residence on Hsp70 mediated by Hip may prevent aggregation or facilitate substrate transfer to downstream Hsp90 or to the degradation machinery. The decision to fold 
or degrade the client might depend on the competition between downstream co-chaperones, Hop/Sti1 and the ubiquitin ligase Chip. Both of them target the C-terminal EEVD motif of Hsp70 (Scheufler et al., 2000; Zhang et al., 2005). Previous studies showed that Hip cooperated with both factors. Hop and Hip synergize in the maturation of GR (Nelson et al., 2004) and Hip and Chip support increased proteasomal degradation of iNOS (Wang et al., 2013).

We have demonstrated that Hip binds to the NBD domain of Hsp70. It was known that Hop binds to the SBD of Hsp70. Thus a simultaneous interaction of Hop and Hip with Hsp70 seems possible. Consistently, a complex containing Hsp70, Hsp90, Hip and Hop has been identified as an intermediate during progesterone receptor activation (Smith et al., 1995). Besides Hop, Chip also interacts with Hsp70 and plays an essential role in balancing substrate protein folding and degradation. It was reported that Chip associated with Hsp70 and blocked the ATPase cycle of the chaperone (Ballinger et al., 1999). Apparently Hsp70 selects substrates for Chipmediated ubiquitylation. Thereby Chip might associate with Hsp70 to promote the ubiquitylation of diverse substrates.

The balance between the diverse pathways, active protein folding by Hsp70, hand-over to Hsp90 or to the protein degradation machinery, apart from the cellular concentrations of the components and phosphate levels - might also be regulated by the post-translational modifications of Hip. Phosphorylation of Hip at serine residues 74, 75 and 78 adjacent to its Hsp70binding domain and within the DP domain likely modulates the activity of Hip, providing additional regulation of substrate flux (Barker and Benovic, 2011; Olsen et al., 2006).

Both Hop and Chip could also be involved in the interaction of Hip with Hsp70 and substrate, but the underlying mechanism remains elusive. One possibility to study the simultaneous interactions of Hip-Hsp70 with Hop or Chip in detail would be to determine the crystal structures of complexes of the full-length proteins. 


\section{Implications and outlook}

Determination of three-dimensional structures of Hip and the Hip-Hsp70 core complex was the main achievement in this study. We presented the crystal structures of the N-terminal dimerization domain of Hip, the TPR binding domain for Hsp70 and the core complex with the ATPase domain of Hsp70. Thus, this work provides novel insights into the function of Hip at the molecular level.

The biochemical and structural data presented in this work lead to a detailed model for the function of metazoan Hsp70 and provide a preliminary model of the regulatory role of Hip in protein quality control. Hip may function as a central scaffolding protein that coordinates the interplay between multiple chaperones such as Hsp40, Hsp70 and Hsp90 and the proteasomal degradation machinery. Since Hip associates with Hsp70-substrate complexes, an important and interesting future goal will be to identify the client proteins of Hip. If the substrates include signaling molecules known to interact with Hsp90, this would provide clear evidence that Hip contributes to the conformational regulation of these clients and cooperates with Hsp90 in their biogenesis.

Increasing the level of Hip has been shown to reduce pathologic protein aggregation in cellular models of Parkinson's and Huntington's disease (Howarth et al., 2009; Roodveldt et al., 2009). Enhancing Hip activity or mimicking its effect on Hsp70 pharmacologically (Wang et al., 2013) may prove useful in facilitating the proteolytic clearance of toxic, aggregation-prone proteins. Similarly, boosting Hip function might be advantageous in cancer therapy (Zhou et al., 2012) by accelerating the clearance of metastable, oncogenic mutant proteins.

Given that mammalian cells likely possess multiple isoforms of J-proteins and different types of NEFs in the same compartment, controlling the ATPase activity of Hsp70 appears to be one central determinant for regulation of chaperone activity. Hip competes with NEFs for the binding to the ADP state of Hsp70, which might have far-reaching effects. It will be interesting to see whether retarding ADP-release from Hsp70 is also utilized more extensively in the regulation of protein quality control. This might create new opportunities for drug design and therapeutic interventions. 


\section{Reference}

Ali, M.M., Roe, S.M., Vaughan, C.K., Meyer, P., Panaretou, B., Piper, P.W., Prodromou, C., and Pearl, L.H. (2006). Crystal structure of an Hsp90-nucleotide-p23/Sba1 closed chaperone complex. Nature 440, 1013-1017.

Anfinsen, C.B. (1973). Principles that govern the folding of protein chains. Science 181, 223230.

Anfinsen, C.B., and Haber, E. (1961). Studies on the reduction and re-formation of protein disulfide bonds. The Journal of biological chemistry 236, 1361-1363.

Anfinsen, C.B., Redfield, R.R., Choate, W.L., Page, J., and Carroll, W.R. (1954). Studies on the gross structure, cross-linkages, and terminal sequences in ribonuclease. The Journal of biological chemistry 207, 201-210.

Anttonen, A.K., Mahjneh, I., Hamalainen, R.H., Lagier-Tourenne, C., Kopra, O., Waris, L., Anttonen, M., Joensuu, T., Kalimo, H., Paetau, A., et al. (2005). The gene disrupted in Marinesco-Sjogren syndrome encodes SIL1, an HSPA5 cochaperone. Nature genetics 37, 1309-1311.

Arakawa, A., Handa, N., Ohsawa, N., Shida, M., Kigawa, T., Hayashi, F., Shirouzu, M., and Yokoyama, S. (2010). The C-terminal BAG domain of BAG5 induces conformational changes of the Hsp70 nucleotide-binding domain for ADP-ATP exchange. Structure 18, 309-319.

Arakawa, A., Handa, N., Shirouzu, M., and Yokoyama, S. (2011). Biochemical and structural studies on the high affinity of Hsp70 for ADP. Protein science : a publication of the Protein Society 20, 1367-1379.

Ballinger, C.A., Connell, P., Wu, Y., Hu, Z., Thompson, L.J., Yin, L.Y., and Patterson, C. (1999). Identification of CHIP, a novel tetratricopeptide repeat-containing protein that interacts with heat shock proteins and negatively regulates chaperone functions. Molecular and cellular biology 19, 4535-4545.

Barker, B.L., and Benovic, J.L. (2011). G protein-coupled receptor kinase 5 phosphorylation of hip regulates internalization of the chemokine receptor CXCR4. Biochemistry 50, 6933-6941.

Barthel, T.K., Zhang, J., and Walker, G.C. (2001). ATPase-defective derivatives of Escherichia coli DnaK that behave differently with respect to ATP-induced conformational change and peptide release. Journal of bacteriology 183, 5482-5490.

Battye, T.G., Kontogiannis, L., Johnson, O., Powell, H.R., and Leslie, A.G. (2011). iMOSFLM: a new graphical interface for diffraction-image processing with MOSFLM. Acta crystallographica Section D, Biological crystallography 67, 271-281.

Bose, S., Weikl, T., Bugl, H., and Buchner, J. (1996). Chaperone function of Hsp90-associated proteins. Science 274, 1715-1717. 
Brehmer, D., Gassler, C., Rist, W., Mayer, M.P., and Bukau, B. (2004). Influence of GrpE on DnaK-substrate interactions. The Journal of biological chemistry 279, 27957-27964.

Brehmer, D., Rudiger, S., Gassler, C.S., Klostermeier, D., Packschies, L., Reinstein, J., Mayer, M.P., and Bukau, B. (2001). Tuning of chaperone activity of Hsp70 proteins by modulation of nucleotide exchange. Nature structural biology 8, 427-432.

Brodsky, J.L., and Chiosis, G. (2006). Hsp70 molecular chaperones: emerging roles in human disease and identification of small molecule modulators. Current topics in medicinal chemistry $6,1215-1225$.

Bruce, B.D., and Churchich, J. (1997). Characterization of the molecular-chaperone function of the heat-shock-cognate-70-interacting protein. European journal of biochemistry / FEBS 245, 738-744.

Buchberger, A., Theyssen, H., Schroder, H., McCarty, J.S., Virgallita, G., Milkereit, P., Reinstein, J., and Bukau, B. (1995). Nucleotide-induced conformational changes in the ATPase and substrate binding domains of the DnaK chaperone provide evidence for interdomain communication. The Journal of biological chemistry 270, 16903-16910.

Buchner, J. (1999). Hsp90 \& Co. - a holding for folding. Trends in biochemical sciences 24, 136-141.

Burrows, F., Zhang, H., and Kamal, A. (2004). Hsp90 activation and cell cycle regulation. Cell cycle $3,1530-1536$.

Caplan, A.J. (1999). Hsp90's secrets unfold: new insights from structural and functional studies. Trends in cell biology 9, 262-268.

Carrigan, P.E., Nelson, G.M., Roberts, P.J., Stoffer, J., Riggs, D.L., and Smith, D.F. (2004). Multiple domains of the co-chaperone Hop are important for Hsp70 binding. The Journal of biological chemistry 279, 16185-16193.

Chang, H.C., and Lindquist, S. (1994). Conservation of Hsp90 macromolecular complexes in Saccharomyces cerevisiae. The Journal of biological chemistry 269, 24983-24988.

Chang, Y.W., Sun, Y.J., Wang, C., and Hsiao, C.D. (2008). Crystal structures of the 70-kDa heat shock proteins in domain disjoining conformation. The Journal of biological chemistry $283,15502-15511$.

Cheetham, M.E., and Caplan, A.J. (1998). Structure, function and evolution of DnaJ: conservation and adaptation of chaperone function. Cell stress \& chaperones 3, 28-36.

Chen, S., Prapapanich, V., Rimerman, R.A., Honore, B., and Smith, D.F. (1996). Interactions of p60, a mediator of progesterone receptor assembly, with heat shock proteins hsp90 and hsp70. Molecular endocrinology 10, 682-693.

Chen, S., and Smith, D.F. (1998). Hop as an adaptor in the heat shock protein 70 (Hsp70) and hsp90 chaperone machinery. The Journal of biological chemistry 273, 35194-35200. 
Chen, S., Sullivan, W.P., Toft, D.O., and Smith, D.F. (1998). Differential interactions of p23 and the TPR-containing proteins Hop, Cyp40, FKBP52 and FKBP51 with Hsp90 mutants. Cell stress \& chaperones 3, 118-129.

Chiti, F., and Dobson, C.M. (2006). Protein misfolding, functional amyloid, and human disease. Annual review of biochemistry 75, 333-366.

Collaborative Computational Project, N. (1994). The CCP4 suite: programs for protein crystallography. Acta crystallographica Section D, Biological crystallography 50, 760-763.

Connell, P., Ballinger, C.A., Jiang, J., Wu, Y., Thompson, L.J., Hohfeld, J., and Patterson, C. (2001). The co-chaperone CHIP regulates protein triage decisions mediated by heat-shock proteins. Nature cell biology 3, 93-96.

Corpet, F. (1988). Multiple sequence alignment with hierarchical clustering. Nucleic acids research 16, 10881-10890.

D'Andrea, L.D., and Regan, L. (2003). TPR proteins: the versatile helix. Trends Biochem Sci $28,655-662$.

Dedmon, M.M., Christodoulou, J., Wilson, M.R., and Dobson, C.M. (2005). Heat shock protein 70 inhibits alpha-synuclein fibril formation via preferential binding to prefibrillar species. The Journal of biological chemistry 280, 14733-14740.

Demand, J., Luders, J., and Höhfeld, J. (1998). The carboxy-terminal domain of Hsc70 provides binding sites for a distinct set of chaperone cofactors. Molecular and cellular biology $18,2023-2028$.

Deocaris, C.C., Widodo, N., Shrestha, B.G., Kaur, K., Ohtaka, M., Yamasaki, K., Kaul, S.C., and Wadhwa, R. (2007). Mortalin sensitizes human cancer cells to MKT-077-induced senescence. Cancer letters 252, 259-269.

Dolinski, K.J., Cardenas, M.E., and Heitman, J. (1998). CNS1 encodes an essential p60/Sti1 homolog in Saccharomyces cerevisiae that suppresses cyclophilin 40 mutations and interacts with Hsp90. Molecular and cellular biology 18, 7344-7352.

Dores-Silva, P.R., Silva, E.R., Gomes, F.E., Silva, K.P., Barbosa, L.R., and Borges, J.C. (2012). Low resolution structural characterization of the Hsp70-interacting protein - Hip - from Leishmania braziliensis emphasizes its high asymmetry. Arch Biochem Biophys 520, 88-98.

Dragovic, Z., Broadley, S.A., Shomura, Y., Bracher, A., and Hartl, F.U. (2006). Molecular chaperones of the Hsp110 family act as nucleotide exchange factors of Hsp70s. The EMBO journal 25, 2519-2528.

Easton, D.P., Kaneko, Y., and Subjeck, J.R. (2000). The hsp110 and Grp1 70 stress proteins: newly recognized relatives of the Hsp70s. Cell stress \& chaperones 5, 276-290.

Ellis, J. (1987). Proteins as molecular chaperones. Nature 328, 378-379. 
Ellis, R.J. (2001). Macromolecular crowding: an important but neglected aspect of the intracellular environment. Current opinion in structural biology 11, 114-119.

Emsley, P., and Cowtan, K. (2004). Coot: model-building tools for molecular graphics. Acta crystallographica Section D, Biological crystallography 60, 2126-2132.

Eroglu, B., Moskophidis, D., and Mivechi, N.F. (2010). Loss of Hsp110 leads to age-dependent tau hyperphosphorylation and early accumulation of insoluble amyloid beta. Molecular and cellular biology 30, 4626-4643.

Evans, C.G., Chang, L., and Gestwicki, J.E. (2010). Heat shock protein 70 (hsp70) as an emerging drug target. Journal of medicinal chemistry 53, 4585-4602.

Evans, P. (2006). Scaling and assessment of data quality. Acta crystallographica Section D, Biological crystallography $62,72-82$.

Evans, P.R. (1997). SCALA. Jonit CCP4 and ESF-EACBM Newsletter 33, 22-24.

Fan, G.H., Yang, W., Sai, J., and Richmond, A. (2002). Hsc/Hsp70 interacting protein (hip) associates with CXCR2 and regulates the receptor signaling and trafficking. The Journal of biological chemistry 277, 6590-6597.

Flom, G., Behal, R.H., Rosen, L., Cole, D.G., and Johnson, J.L. (2007). Definition of the minimal fragments of Sti1 required for dimerization, interaction with $\mathrm{Hsp} 70$ and $\mathrm{Hsp} 90$ and in vivo functions. The Biochemical journal 404, 159-167.

Flom, G., Weekes, J., Williams, J.J., and Johnson, J.L. (2006). Effect of mutation of the tetratricopeptide repeat and asparatate-proline 2 domains of Sti1 on Hsp90 signaling and interaction in Saccharomyces cerevisiae. Genetics 172, 41-51.

Frydman, J., and Höhfeld, J. (1997). Chaperones get in touch: the Hip-Hop connection. Trends in biochemical sciences $22,87-92$.

Gamer, J., Multhaup, G., Tomoyasu, T., McCarty, J.S., Rudiger, S., Schonfeld, H.J., Schirra, C., Bujard, H., and Bukau, B. (1996). A cycle of binding and release of the DnaK, DnaJ and GrpE chaperones regulates activity of the Escherichia coli heat shock transcription factor sigma32. The EMBO journal 15, 607-617.

Gässler, C.S., Wiederkehr, T., Brehmer, D., Bukau, B., and Mayer, M.P. (2001). Bag-1M accelerates nucleotide release for human Hsc70 and Hsp70 and can act concentrationdependent as positive and negative cofactor. The Journal of biological chemistry 276, 3253832544.

Gietz, R.D., Schiestl, R.H., Willems, A.R., and Woods, R.A. (1995). Studies on the transformation of intact yeast cells by the LiAc/SS-DNA/PEG procedure. Yeast 11, 355-360.

Gomez-Puertas, P., Martin-Benito, J., Carrascosa, J.L., Willison, K.R., and Valpuesta, J.M. (2004). The substrate recognition mechanisms in chaperonins. Journal of molecular recognition : JMR 17, 85-94. 
Gouet, P., Courcelle, E., Stuart, D.I., and Metoz, F. (1999). ESPript: multiple sequence alignments in PostScript. Bioinformatics 15, 305-308.

Gross, M., and Hessefort, S. (1996). Purification and characterization of a 66-kDa protein from rabbit reticulocyte lysate which promotes the recycling of hsp 70. The Journal of biological chemistry $271,16833-16841$.

Hanahan, D. (1983). Studies on transformation of Escherichia coli with plasmids. Journal of molecular biology 166, 557-580.

Harrison, C. (2003). GrpE, a nucleotide exchange factor for DnaK. Cell stress \& chaperones 8 , 218-224.

Harrison, C.J., Hayer-Hartl, M., Di Liberto, M., Hartl, F., and Kuriyan, J. (1997). Crystal structure of the nucleotide exchange factor GrpE bound to the ATPase domain of the molecular chaperone DnaK. Science 276, 431-435.

Hartl, F.U. (1996). Molecular chaperones in cellular protein folding. Nature 381, 571-580.

Hartl, F.U., Bracher, A., and Hayer-Hartl, M. (2011). Molecular chaperones in protein folding and proteostasis. Nature 475, 324-332.

Hartl, F.U., and Hayer-Hartl, M. (2002). Molecular chaperones in the cytosol: from nascent chain to folded protein. Science 295, 1852-1858.

Hartl, F.U., and Hayer-Hartl, M. (2009). Converging concepts of protein folding in vitro and in vivo. Nature structural \& molecular biology 16, 574-581.

Höhfeld, J., and Jentsch, S. (1997). GrpE-like regulation of the Hsc70 chaperone by the antiapoptotic protein BAG-1. Embo Journal 16, 6209-6216.

Höhfeld, J., Minami, Y., and Hartl, F.U. (1995). Hip, a novel cochaperone involved in the eukaryotic Hsc70/Hsp40 reaction cycle. Cell 83, 589-598.

Horwich, A.L., Fenton, W.A., Chapman, E., and Farr, G.W. (2007). Two families of chaperonin: physiology and mechanism. Annual review of cell and developmental biology 23, 115-145.

Howarth, J.L., Glover, C.P., and Uney, J.B. (2009). HSP70 interacting protein prevents the accumulation of inclusions in polyglutamine disease. Journal of neurochemistry 108, 945-951.

Hubbard, S.J., and Thornton, J.M. (1993). NACCESS

Ikeda, E., Yoshida, S., Mitsuzawa, H., Uno, I., and Toh-e, A. (1994). YGE1 is a yeast homologue of Escherichia coli grpE and is required for maintenance of mitochondrial functions. FEBS letters 339, 265-268.

Irmer, H., and Höhfeld, J. (1997). Characterization of functional domains of the eukaryotic cochaperone Hip. The Journal of biological chemistry 272, 2230-2235. 
Jana, N.R., and Nukina, N. (2005). BAG-1 associates with the polyglutamine-expanded huntingtin aggregates. Neuroscience letters 378, 171-175.

Jana, N.R., Tanaka, M., Wang, G., and Nukina, N. (2000). Polyglutamine length-dependent interaction of Hsp40 and Hsp70 family chaperones with truncated N-terminal huntingtin: their role in suppression of aggregation and cellular toxicity. Human molecular genetics 9, 20092018.

Jiang, J., Maes, E.G., Taylor, A.B., Wang, L., Hinck, A.P., Lafer, E.M., and Sousa, R. (2007). Structural basis of J cochaperone binding and regulation of Hsp70. Molecular cell 28, 422-433.

Kabani, M., McLellan, C., Raynes, D.A., Guerriero, V., and Brodsky, J.L. (2002). HspBP1, a homologue of the yeast Fes 1 and Sls1 proteins, is an Hsc70 nucleotide exchange factor. FEBS letters 531, 339-342.

Kabbage, M., and Dickman, M.B. (2008). The BAG proteins: a ubiquitous family of chaperone regulators. Cellular and molecular life sciences : CMLS 65, 1390-1402.

Kabsch, W. (2010). Integration, scaling, space-group assignment and post-refinement. Acta crystallographica Section D, Biological crystallography 66, 133-144.

Karzai, A.W., and McMacken, R. (1996). A bipartite signaling mechanism involved in DnaJmediated activation of the Escherichia coli DnaK protein. The Journal of biological chemistry 271, 11236-11246.

Kerner, M.J., Naylor, D.J., Ishihama, Y., Maier, T., Chang, H.C., Stines, A.P., Georgopoulos, C., Frishman, D., Hayer-Hartl, M., Mann, M., et al. (2005). Proteome-wide analysis of chaperonin-dependent protein folding in Escherichia coli. Cell 122, 209-220.

Kim, Y.E., Hipp, M.S., Bracher, A., Hayer-Hartl, M., and Hartl, F.U. (2013). Molecular chaperone functions in protein folding and proteostasis. Annu Rev Biochem 82, 323-355.

Kim, Y.S., Alarcon, S.V., Lee, S., Lee, M.J., Giaccone, G., Neckers, L., and Trepel, J.B. (2009). Update on Hsp90 inhibitors in clinical trial. Current topics in medicinal chemistry 9, 1479-1492.

Kirkegaard, T., Roth, A.G., Petersen, N.H., Mahalka, A.K., Olsen, O.D., Moilanen, I., Zylicz, A., Knudsen, J., Sandhoff, K., Arenz, C., et al. (2010). Hsp70 stabilizes lysosomes and reverts Niemann-Pick disease-associated lysosomal pathology. Nature 463, 549-553.

Kityk, R., Kopp, J., Sinning, I., and Mayer, M.P. (2012). Structure and dynamics of the ATPbound open conformation of Hsp70 chaperones. Molecular cell 48, 863-874.

Kleywegt, G.T., and Jones, T.A. (1994). A super position. CCP4/ESF-EACBM Newsletter on Protein Crystallography 31, 9-14.

Konarev, P.V., Volkov, V.V., Sokolova, A.V., Koch, M.H.J., and Svergun, D.I. (2003). PRIMUS: a Windows PC-based system for small-angle scattering data analysis. J Appl Crystallogr 36, 1277-1282. 
Kose, S., Furuta, M., and Imamoto, N. (2012). Hikeshi, a nuclear import carrier for Hsp70s, protects cells from heat shock-induced nuclear damage. Cell 149, 578-589.

Kota, P., Summers, D.W., Ren, H.Y., Cyr, D.M., and Dokholyan, N.V. (2009). Identification of a consensus motif in substrates bound by a Type I Hsp40. Proceedings of the National Academy of Sciences of the United States of America 106, 11073-11078.

Koya, K., Li, Y., Wang, H., Ukai, T., Tatsuta, N., Kawakami, M., Shishido, and Chen, L.B. (1996). MKT-077, a novel rhodacyanine dye in clinical trials, exhibits anticarcinoma activity in preclinical studies based on selective mitochondrial accumulation. Cancer research 56, 538-543.

Krobitsch, S., and Lindquist, S. (2000). Aggregation of huntingtin in yeast varies with the length of the polyglutamine expansion and the expression of chaperone proteins. Proceedings of the National Academy of Sciences of the United States of America 97, 1589-1594.

Kushnirov, V.V. (2000). Rapid and reliable protein extraction from yeast. Yeast 16, 857-860.

Laemmli, U.K. (1970). Cleavage of structural proteins during the assembly of the head of bacteriophage T4. Nature 227, 680-685.

Laloraya, S., Gambill, B.D., and Craig, E.A. (1994). A role for a eukaryotic GrpE-related protein, Mge1p, in protein translocation. Proceedings of the National Academy of Sciences of the United States of America 91, 6481-6485.

Lamb, J.R., Tugendreich, S., and Hieter, P. (1995). Tetratrico peptide repeat interactions: to TPR or not to TPR? Trends in biochemical sciences 20, 257-259.

Landry, S.J. (2003). Structure and energetics of an allele-specific genetic interaction between dnaJ and dnaK: correlation of nuclear magnetic resonance chemical shift perturbations in the Jdomain of Hsp40/DnaJ with binding affinity for the ATPase domain of Hsp70/DnaK.

Biochemistry 42, 4926-4936.

Larkin, M.A., Blackshields, G., Brown, N.P., Chenna, R., McGettigan, P.A., McWilliam, H., Valentin, F., Wallace, I.M., Wilm, A., Lopez, R., et al. (2007). Clustal W and Clustal X version 2.0. Bioinformatics 23, 2947-2948.

Laufen, T., Mayer, M.P., Beisel, C., Klostermeier, D., Mogk, A., Reinstein, J., and Bukau, B. (1999). Mechanism of regulation of hsp70 chaperones by DnaJ cochaperones. Proceedings of the National Academy of Sciences of the United States of America 96, 5452-5457.

Lawrence, M.C., and Colman, P.M. (1993). Shape complementarity at protein/protein interfaces. Journal of molecular biology 234, 946-950.

Lee, B., and Richards, F.M. (1971). The interpretation of protein structures: estimation of static accessibility. Journal of molecular biology 55, 379-400.

Lindquist, S., and Craig, E.A. (1988). The heat-shock proteins. Annual review of genetics 22, 631-677. 
Liu, Q., and Hendrickson, W.A. (2007). Insights into Hsp70 chaperone activity from a crystal structure of the yeast Hsp110 Sse1. Cell 131, 106-120.

Liu, Y., Gierasch, L.M., and Bahar, I. (2010). Role of Hsp70 ATPase domain intrinsic dynamics and sequence evolution in enabling its functional interactions with NEFs. PLoS Comput Biol 6.

Lüders, J., Demand, J., and Höhfeld, J. (2000). The ubiquitin-related BAG-1 provides a link between the molecular chaperones Hsc70/Hsp70 and the proteasome. The Journal of biological chemistry 275, 4613-4617.

Martin, J., and Hartl, F.U. (1997). The effect of macromolecular crowding on chaperoninmediated protein folding. Proceedings of the National Academy of Sciences of the United States of America 94, 1107-1112.

Mayer, M.P. (2010). Gymnastics of molecular chaperones. Molecular cell 39, 321-331.

Mayer, M.P., and Bukau, B. (2005). Hsp70 chaperones: cellular functions and molecular mechanism. Cellular and molecular life sciences : CMLS 62, 670-684.

Mayer, M.P., Schroder, H., Rudiger, S., Paal, K., Laufen, T., and Bukau, B. (2000). Multistep mechanism of substrate binding determines chaperone activity of Hsp70. Nature structural biology 7, 586-593.

McCarty, J.S., and Walker, G.C. (1991). DnaK as a thermometer: threonine-199 is site of autophosphorylation and is critical for ATPase activity. Proceedings of the National Academy of Sciences of the United States of America 88, 9513-9517.

McClellan, A.J., Xia, Y., Deutschbauer, A.M., Davis, R.W., Gerstein, M., and Frydman, J. (2007). Diverse cellular functions of the Hsp90 molecular chaperone uncovered using systems approaches. Cell 131, 121-135.

Meacham, G.C., Patterson, C., Zhang, W., Younger, J.M., and Cyr, D.M. (2001). The Hsc70 co-chaperone CHIP targets immature CFTR for proteasomal degradation. Nature cell biology 3 , 100-105.

Min, J.N., Whaley, R.A., Sharpless, N.E., Lockyer, P., Portbury, A.L., and Patterson, C. (2008). CHIP deficiency decreases longevity, with accelerated aging phenotypes accompanied by altered protein quality control. Molecular and cellular biology 28, 4018-4025.

Minton, A.P. (2000). Implications of macromolecular crowding for protein assembly. Current opinion in structural biology 10, 34-39.

Misselwitz, B., Staeck, O., and Rapoport, T.A. (1998). J proteins catalytically activate Hsp70 molecules to trap a wide range of peptide sequences. Molecular cell 2, 593-603.

Morishima, Y., Kanelakis, K.C., Silverstein, A.M., Dittmar, K.D., Estrada, L., and Pratt, W.B. (2000). The Hsp organizer protein hop enhances the rate of but is not essential for 
glucocorticoid receptor folding by the multiprotein Hsp90-based chaperone system. The Journal of biological chemistry 275, 6894-6900.

Muchowski, P.J., Schaffar, G., Sittler, A., Wanker, E.E., Hayer-Hartl, M.K., and Hartl, F.U. (2000). Hsp70 and hsp40 chaperones can inhibit self-assembly of polyglutamine proteins into amyloid-like fibrils. Proceedings of the National Academy of Sciences of the United States of America 97, 7841-7846.

Mukai, H., Kuno, T., Tanaka, H., Hirata, D., Miyakawa, T., and Tanaka, C. (1993). Isolation and characterization of SSE1 and SSE2, new members of the yeast HSP70 multigene family. Gene 132, 57-66.

Mumberg, D., Muller, R., and Funk, M. (1995). Yeast vectors for the controlled expression of heterologous proteins in different genetic backgrounds. Gene 156, 119-122.

Murshudov, G.N., Vagin, A.A., and Dodson, E.J. (1997). Refinement of macromolecular structures by the maximum-likelihood method. Acta crystallographica Section D, Biological crystallography 53, 240-255.

Nelson, G.M., Huffman, H., and Smith, D.F. (2003). Comparison of the carboxy-terminal DPrepeat region in the co-chaperones Hop and Hip. Cell stress \& chaperones 8, 125-133.

Nelson, G.M., Prapapanich, V., Carrigan, P.E., Roberts, P.J., Riggs, D.L., and Smith, D.F. (2004). The heat shock protein 70 cochaperone hip enhances functional maturation of glucocorticoid receptor. Molecular endocrinology 18, 1620-1630.

Nelson, R.J., Ziegelhoffer, T., Nicolet, C., Werner-Washburne, M., and Craig, E.A. (1992). The translation machinery and $70 \mathrm{kd}$ heat shock protein cooperate in protein synthesis. Cell 71, 97105 .

Neupert, W., and Brunner, M. (2002). The protein import motor of mitochondria. Nature reviews Molecular cell biology 3, 555-565.

Nicolet, C.M., and Craig, E.A. (1989). Isolation and characterization of STI1, a stress-inducible gene from Saccharomyces cerevisiae. Molecular and cellular biology 9, 3638-3646.

Nollen, E.A., Kabakov, A.E., Brunsting, J.F., Kanon, B., Höhfeld, J., and Kampinga, H.H. (2001). Modulation of in vivo HSP70 chaperone activity by Hip and Bag-1. J Biol Chem 276, 4677-4682.

Oh, H.J., Chen, X., and Subjeck, J.R. (1997). Hsp110 protects heat-denatured proteins and confers cellular thermoresistance. The Journal of biological chemistry 272, 31636-31640.

Oh, H.J., Easton, D., Murawski, M., Kaneko, Y., and Subjeck, J.R. (1999). The chaperoning activity of hsp110. Identification of functional domains by use of targeted deletions. The Journal of biological chemistry 274, 15712-15718. 
Olsen, J.V., Blagoev, B., Gnad, F., Macek, B., Kumar, C., Mortensen, P., and Mann, M. (2006). Global, in vivo, and site-specific phosphorylation dynamics in signaling networks. Cell 127, 635-648.

Olsen, J.V., Vermeulen, M., Santamaria, A., Kumar, C., Miller, M.L., Jensen, L.J., Gnad, F., Cox, J., Jensen, T.S., Nigg, E.A., et al. (2010). Quantitative phosphoproteomics reveals widespread full phosphorylation site occupancy during mitosis. Science signaling 3, ra3.

Olzscha, H., Schermann, S.M., Woerner, A.C., Pinkert, S., Hecht, M.H., Tartaglia, G.G., Vendruscolo, M., Hayer-Hartl, M., Hartl, F.U., and Vabulas, R.M. (2011). Amyloid-like aggregates sequester numerous metastable proteins with essential cellular functions. Cell 144, 67-78.

Patury, S., Miyata, Y., and Gestwicki, J.E. (2009). Pharmacological targeting of the Hsp70 chaperone. Current topics in medicinal chemistry 9, 1337-1351.

Pearl, L.H., and Prodromou, C. (2000). Structure and in vivo function of Hsp90. Current opinion in structural biology $10,46-51$.

Picard, D. (2006). Chaperoning steroid hormone action. Trends in endocrinology and metabolism: TEM 17, 229-235.

Pierpaoli, E.V., Sandmeier, E., Baici, A., Schonfeld, H.J., Gisler, S., and Christen, P. (1997). The power stroke of the DnaK/DnaJ/GrpE molecular chaperone system. Journal of molecular biology 269, 757-768.

Pitsis, A.A., and Visouli, A.N. (2011). Mechanical assistance of the circulation during cardiogenic shock. Current opinion in critical care 17, 425-438.

Place, S.P. (2011). Single-point mutation in a conserved TPR domain of Hip disrupts enhancement of glucocorticoid receptor signaling. Cell stress \& chaperones 16, 469-474.

Polier, S., Dragovic, Z., Hartl, F.U., and Bracher, A. (2008). Structural basis for the cooperation of Hsp70 and Hsp110 chaperones in protein folding. Cell 133, 1068-1079.

Polier, S., Hartl, F.U., and Bracher, A. (2010). Interaction of the Hsp110 molecular chaperones from S. cerevisiae with substrate protein. Journal of molecular biology 401, 696-707.

Powers, M.V., Clarke, P.A., and Workman, P. (2008). Dual targeting of HSC70 and HSP72 inhibits HSP90 function and induces tumor-specific apoptosis. Cancer cell 14, 250-262.

Prapapanich, V., Chen, S., Nair, S.C., Rimerman, R.A., and Smith, D.F. (1996a). Molecular cloning of human $\mathrm{p} 48$, a transient component of progesterone receptor complexes and an Hsp70-binding protein. Molecular endocrinology 10, 420-431.

Prapapanich, V., Chen, S., and Smith, D.F. (1998). Mutation of Hip's carboxy-terminal region inhibits a transitional stage of progesterone receptor assembly. Molecular and cellular biology 18, 944-952. 
Prapapanich, V., Chen, S., Toran, E.J., Rimerman, R.A., and Smith, D.F. (1996b). Mutational analysis of the hsp70-interacting protein Hip. Molecular and cellular biology 16, 6200-6207.

Pratt, W.B., and Toft, D.O. (1997). Steroid receptor interactions with heat shock protein and immunophilin chaperones. Endocrine reviews 18, 306-360.

Pratt, W.B., and Toft, D.O. (2003). Regulation of signaling protein function and trafficking by the hsp90/hsp70-based chaperone machinery. Experimental biology and medicine 228, 111-133.

Qi, R., Sarbeng, E.B., Liu, Q., Le, K.Q., Xu, X., Xu, H., Yang, J., Wong, J.L., Vorvis, C., Hendrickson, W.A., et al. (2013). Allosteric opening of the polypeptide-binding site when an Hsp70 binds ATP. Nature structural \& molecular biology.

Qiu, X.B., Shao, Y.M., Miao, S., and Wang, L. (2006). The diversity of the DnaJ/Hsp40 family, the crucial partners for Hsp70 chaperones. Cellular and molecular life sciences : CMLS 63, 2560-2570.

Rampelt, H., Kirstein-Miles, J., Nillegoda, N.B., Chi, K., Scholz, S.R., Morimoto, R.I., and Bukau, B. (2012). Metazoan Hsp70 machines use Hsp110 to power protein disaggregation. The EMBO journal 31, 4221-4235.

Raviol, H., Sadlish, H., Rodriguez, F., Mayer, M.P., and Bukau, B. (2006). Chaperone network in the yeast cytosol: Hsp110 is revealed as an Hsp70 nucleotide exchange factor. EMBO J 25, 2510-2518.

Raynes, D.A., and Guerriero, V., Jr. (1998). Inhibition of Hsp70 ATPase activity and protein renaturation by a novel Hsp70-binding protein. The Journal of biological chemistry 273, 32883-32888.

Rehm, T., Huber, R., and Holak, T.A. (2002). Application of NMR in structural proteomics: screening for proteins amenable to structural analysis. Structure 10, 1613-1618.

Riggs, D.L., Roberts, P.J., Chirillo, S.C., Cheung-Flynn, J., Prapapanich, V., Ratajczak, T., Gaber, R., Picard, D., and Smith, D.F. (2003). The Hsp90-binding peptidylprolyl isomerase FKBP52 potentiates glucocorticoid signaling in vivo. EMBO J 22, 1158-1167.

Roodveldt, C., Bertoncini, C.W., Andersson, A., van der Goot, A.T., Hsu, S.T., FernandezMontesinos, R., de Jong, J., van Ham, T.J., Nollen, E.A., Pozo, D., et al. (2009). Chaperone proteostasis in Parkinson's disease: stabilization of the Hsp70/alpha-synuclein complex by Hip. The EMBO journal 28, 3758-3770.

Rousaki, A., Miyata, Y., Jinwal, U.K., Dickey, C.A., Gestwicki, J.E., and Zuiderweg, E.R. (2011). Allosteric drugs: the interaction of antitumor compound MKT-077 with human Hsp70 chaperones. Journal of molecular biology 411, 614-632.

Rubinsztein, D.C. (2006). The roles of intracellular protein-degradation pathways in neurodegeneration. Nature 443, 780-786. 
Ryan, M.T., and Pfanner, N. (2001). Hsp70 proteins in protein translocation. Advances in protein chemistry $59,223-242$.

Sambrook, J., Fritsch, E. F., and Maniatis, T. (1989). Molecular Cloning - A laboratory manual. Cold Spring Harbor Laboratory press.

Sanchez, E.R., Toft, D.O., Schlesinger, M.J., and Pratt, W.B. (1985). Evidence that the 90-kDa phosphoprotein associated with the untransformed L-cell glucocorticoid receptor is a murine heat shock protein. The Journal of biological chemistry 260, 12398-12401.

Scherzer, C.R., Eklund, A.C., Morse, L.J., Liao, Z., Locascio, J.J., Fefer, D., Schwarzschild, M.A., Schlossmacher, M.G., Hauser, M.A., Vance, J.M., et al. (2007). Molecular markers of early Parkinson's disease based on gene expression in blood. Proceedings of the National Academy of Sciences of the United States of America 104, 955-960.

Scheufler, C., Brinker, A., Bourenkov, G., Pegoraro, S., Moroder, L., Bartunik, H., Hartl, F.U., and Moarefi, I. (2000). Structure of TPR domain-peptide complexes: critical elements in the assembly of the Hsp70-Hsp90 multichaperone machine. Cell 101, 199-210.

Schiestl, R.H., and Gietz, R.D. (1989). High efficiency transformation of intact yeast cells using single stranded nucleic acids as a carrier. Current genetics 16, 339-346.

Schmid, A.B., Lagleder, S., Grawert, M.A., Rohl, A., Hagn, F., Wandinger, S.K., Cox, M.B., Demmer, O., Richter, K., Groll, M., et al. (2012). The architecture of functional modules in the Hsp90 co-chaperone Sti1/Hop. The EMBO journal 31, 1506-1517.

Schmid, D., Baici, A., Gehring, H., and Christen, P. (1994). Kinetics of molecular chaperone action. Science 263, 971-973.

Schmid, F.X. (1993). Prolyl isomerase: enzymatic catalysis of slow protein-folding reactions. Annual review of biophysics and biomolecular structure 22, 123-142.

Schneider, C., Sepp-Lorenzino, L., Nimmesgern, E., Ouerfelli, O., Danishefsky, S., Rosen, N., and Hartl, F.U. (1996). Pharmacologic shifting of a balance between protein refolding and degradation mediated by Hsp90. Proceedings of the National Academy of Sciences of the United States of America 93, 14536-14541.

Schneider, T.R., and Sheldrick, G.M. (2002). Substructure solution with SHELXD. Acta crystallographica Section D, Biological crystallography 58, 1772-1779.

Schrödinger, L. (2010). The PyMOL Molecular Graphics System. Version 11r2pre.

Schuermann, J.P., Jiang, J., Cuellar, J., Llorca, O., Wang, L., Gimenez, L.E., Jin, S., Taylor, A.B., Demeler, B., Morano, K.A., et al. (2008). Structure of the Hsp110:Hsc70 nucleotide exchange machine. Molecular cell 31, 232-243.

Senderek, J., Krieger, M., Stendel, C., Bergmann, C., Moser, M., Breitbach-Faller, N., RudnikSchoneborn, S., Blaschek, A., Wolf, N.I., Harting, I., et al. (2005). Mutations in SIL1 cause 
Marinesco-Sjogren syndrome, a cerebellar ataxia with cataract and myopathy. Nature genetics 37, 1312-1314.

Shaner, L., and Morano, K.A. (2007). All in the family: atypical Hsp70 chaperones are conserved modulators of Hsp70 activity. Cell stress \& chaperones 12, 1-8.

Sharp, S., and Workman, P. (2006). Inhibitors of the HSP90 molecular chaperone: current status. Advances in cancer research 95, 323-348.

Shiau, A.K., Harris, S.F., Southworth, D.R., and Agard, D.A. (2006). Structural Analysis of E. coli hsp90 reveals dramatic nucleotide-dependent conformational rearrangements. Cell 127, 329-340.

Shomura, Y., Dragovic, Z., Chang, H.C., Tzvetkov, N., Young, J.C., Brodsky, J.L., Guerriero, V., Hartl, F.U., and Bracher, A. (2005). Regulation of Hsp70 function by HspBP1: structural analysis reveals an alternate mechanism for Hsp70 nucleotide exchange. Mol Cell 17, 367-379.

Skarzynski, T. (1988). Contact.

Smith, D.F. (1993). Dynamics of heat shock protein 90-progesterone receptor binding and the disactivation loop model for steroid receptor complexes. Molecular endocrinology 7, 14181429.

Smith, D.F., Sullivan, W.P., Marion, T.N., Zaitsu, K., Madden, B., McCormick, D.J., and Toft, D.O. (1993). Identification of a 60-kilodalton stress-related protein, p60, which interacts with hsp90 and hsp70. Molecular and cellular biology 13, 869-876.

Smith, D.F., Whitesell, L., Nair, S.C., Chen, S., Prapapanich, V., and Rimerman, R.A. (1995). Progesterone receptor structure and function altered by geldanamycin, an hsp90-binding agent. Molecular and cellular biology 15, 6804-6812.

Sondermann, H., Scheufler, C., Schneider, C., Hohfeld, J., Hartl, F.U., and Moarefi, I. (2001). Structure of a Bag/Hsc70 complex: convergent functional evolution of Hsp70 nucleotide exchange factors. Science 291, 1553-1557.

Stankiewicz, M., Nikolay, R., Rybin, V., and Mayer, M.P. (2010). CHIP participates in protein triage decisions by preferentially ubiquitinating Hsp70-bound substrates. The FEBS journal 277, 3353-3367.

Svergun, D.I. (1992). Determination of the Regularization Parameter in Indirect-Transform Methods Using Perceptual Criteria. J Appl Crystallogr 25, 495-503.

Swain, J.F., Dinler, G., Sivendran, R., Montgomery, D.L., Stotz, M., and Gierasch, L.M. (2007). Hsp70 chaperone ligands control domain association via an allosteric mechanism mediated by the interdomain linker. Molecular cell 26, 27-39.

Taipale, M., Jarosz, D.F., and Lindquist, S. (2010). HSP90 at the hub of protein homeostasis: emerging mechanistic insights. Nature reviews Molecular cell biology 11, 515-528. 
Takayama, S., Sato, T., Krajewski, S., Kochel, K., Irie, S., Millan, J.A., and Reed, J.C. (1995). Cloning and functional analysis of BAG-1: a novel Bcl-2-binding protein with anti-cell death activity. Cell 80, 279-284.

Tang, Y.C., Chang, H.C., Hayer-Hartl, M., and Hartl, F.U. (2007). SnapShot: molecular chaperones, Part II. Cell 128, 412.

Terwilliger, T.C. (2000). Maximum-likelihood density modification. Acta crystallographica Section D, Biological crystallography 56, 965-972.

Thompson, J.D., Higgins, D.G., and Gibson, T.J. (1994). CLUSTAL W: improving the sensitivity of progressive multiple sequence alignment through sequence weighting, positionspecific gap penalties and weight matrix choice. Nucleic Acids Res 22, 4673-4680.

Tuite, M.F., and Freedman, R.B. (1994). Improving secretion of recombinant proteins from yeast and mammalian cells: rational or empirical design? Trends in biotechnology 12, 432-434.

Uparanukraw, P., Toyoshima, T., Aikawa, M., and Kumar, N. (1993). Molecular cloning and localization of an abundant novel protein of Plasmodium berghei. Molecular and biochemical parasitology 59, 223-234.

Urushitani, M., Kurisu, J., Tateno, M., Hatakeyama, S., Nakayama, K., Kato, S., and Takahashi, R. (2004). CHIP promotes proteasomal degradation of familial ALS-linked mutant SOD1 by ubiquitinating Hsp/Hsc70. Journal of neurochemistry 90, 231-244.

Vagin, A.A., and Isupov, M.N. (2001). Spherically averaged phased translation function and its application to the search for molecules and fragments in electron-density maps. Acta crystallographica Section D, Biological crystallography 57, 1451-1456.

Velten, M., Gomez-Vrielynck, N., Chaffotte, A., and Ladjimi, M.M. (2002). Domain structure of the HSC70 cochaperone, HIP. The Journal of biological chemistry 277, 259-266.

Velten, M., Villoutreix, B.O., and Ladjimi, M.M. (2000). Quaternary structure of the HSC70 cochaperone HIP. Biochemistry 39, 307-315.

Vogel, M., Mayer, M.P., and Bukau, B. (2006). Allosteric regulation of Hsp70 chaperones involves a conserved interdomain linker. The Journal of biological chemistry 281, 3870538711.

Vonrhein, C., Blanc, E., Roversi, P., and Bricogne, G. (2007). Automated structure solution with autoSHARP. Methods in molecular biology 364, 215-230.

Vos, M.J., Hageman, J., Carra, S., and Kampinga, H.H. (2008). Structural and functional diversities between members of the human HSPB, HSPH, HSPA, and DNAJ chaperone families. Biochemistry 47, 7001-7011.

Wadhwa, R., Sugihara, T., Yoshida, A., Nomura, H., Reddel, R.R., Simpson, R., Maruta, H., and Kaul, S.C. (2000). Selective toxicity of MKT-077 to cancer cells is mediated by its binding 
to the hsp70 family protein mot- 2 and reactivation of p53 function. Cancer research 60,6818 6821.

Wall, D., Zylicz, M., and Georgopoulos, C. (1994). The NH2-terminal 108 amino acids of the Escherichia coli DnaJ protein stimulate the ATPase activity of DnaK and are sufficient for lambda replication. The Journal of biological chemistry 269, 5446-5451.

Wandinger, S.K., Richter, K., and Buchner, J. (2008). The Hsp90 chaperone machinery. The Journal of biological chemistry 283, 18473-18477.

Wang, A.M., Miyata, Y., Klinedinst, S., Peng, H.M., Chua, J.P., Komiyama, T., Li, X., Morishima, Y., Merry, D.E., Pratt, W.B., et al. (2013). Activation of Hsp70 reduces neurotoxicity by promoting polyglutamine protein degradation. Nat Chem Biol 9, 112-118.

Wegele, H., Haslbeck, M., Reinstein, J., and Buchner, J. (2003). Sti1 is a novel activator of the Ssa proteins. The Journal of biological chemistry 278, 25970-25976.

Weiner, M.P., Costa, G.L., Schoettlin, W., Cline, J., Mathur, E., and Bauer, J.C. (1994). Sitedirected mutagenesis of double-stranded DNA by the polymerase chain reaction. Gene 151, 119-123.

Wilbanks, S.M., and McKay, D.B. (1995). How potassium affects the activity of the molecular chaperone Hsc70. II. Potassium binds specifically in the ATPase active site. J Biol Chem 270, 2251-2257.

Workman, P., Burrows, F., Neckers, L., and Rosen, N. (2007). Drugging the cancer chaperone HSP90: combinatorial therapeutic exploitation of oncogene addiction and tumor stress. Annals of the New York Academy of Sciences 1113, 202-216.

Xu, Z., Horwich, A.L., and Sigler, P.B. (1997). The crystal structure of the asymmetric GroELGroES-(ADP)7 chaperonin complex. Nature 388, 741-750.

Xu, Z., Page, R.C., Gomes, M.M., Kohli, E., Nix, J.C., Herr, A.B., Patterson, C., and Misra, S. (2008). Structural basis of nucleotide exchange and client binding by the Hsp70 cochaperone Bag2. Nat Struct Mol Biol 15, 1309-1317.

Yam, A.Y., Xia, Y., Lin, H.T., Burlingame, A., Gerstein, M., and Frydman, J. (2008). Defining the TRiC/CCT interactome links chaperonin function to stabilization of newly made proteins with complex topologies. Nature structural \& molecular biology 15, 1255-1262.

Yamashita, H., Kawamata, J., Okawa, K., Kanki, R., Nakamizo, T., Hatayama, T., Yamanaka, K., Takahashi, R., and Shimohama, S. (2007). Heat-shock protein 105 interacts with and suppresses aggregation of mutant $\mathrm{Cu} / \mathrm{Zn}$ superoxide dismutase: clues to a possible strategy for treating ALS. Journal of neurochemistry 102, 1497-1505.

Young, J.C., Obermann, W.M., and Hartl, F.U. (1998). Specific binding of tetratricopeptide repeat proteins to the C-terminal 12-kDa domain of hsp90. The Journal of biological chemistry 273, 18007-18010. 
Zhang, M., Windheim, M., Roe, S.M., Peggie, M., Cohen, P., Prodromou, C., and Pearl, L.H. (2005). Chaperoned ubiquitylation--crystal structures of the CHIP U box E3 ubiquitin ligase and a CHIP-Ubc13-Uev1a complex. Molecular cell 20, 525-538.

Zhou, X., Xie, G., Wang, S., Wang, Y., Zhang, K., Zheng, S., Chu, L., Xiao, L., Yu, Y., Zhang, Y., et al. (2012). Potent and specific antitumor effect for colorectal cancer by CEA and Rb double regulated oncolytic adenovirus harboring ST13 gene. PLoS One 7, e47566.

Zhu, X., Zhao, X., Burkholder, W.F., Gragerov, A., Ogata, C.M., Gottesman, M.E., and Hendrickson, W.A. (1996). Structural analysis of substrate binding by the molecular chaperone DnaK. Science 272, 1606-1614.

Zhuravleva, A., and Gierasch, L.M. (2011). Allosteric signal transmission in the nucleotidebinding domain of 70-kDa heat shock protein (Hsp70) molecular chaperones. Proceedings of the National Academy of Sciences of the United States of America 108, 6987-6992.

Ziegelhoffer, T., Johnson, J.L., and Craig, E.A. (1996). Chaperones get Hip. Protein folding. Current biology : CB 6, 272-275. 


\section{Appendix}

\subsection{List of primers}

Oligonucleotides utilized in this study are listed here. Oligonucleotides were purchased from Metabion (Martinsried, Germany).

\begin{tabular}{|c|c|c|c|}
\hline Construct & Primer & Sequence 5' $\rightarrow$ 3' & Endonuclease \\
\hline \multirow[t]{2}{*}{$\operatorname{Hip}(1-44)$} & LZ_HIP1f & CATGCCATGGACCCCCGCAAAGTGAGCGAGC & $\underline{N c o I}$ \\
\hline & LZ_HIP2r & CCCAAGCTTAAGGTGGTACTTTACCCCCCATGCTCTCC & $\underline{\text { HindIIII }}$ \\
\hline \multirow[t]{2}{*}{$\operatorname{Hip}(1-267)$} & $\mathrm{AB} 99 \mathrm{f}$ & $\begin{array}{l}\text { phos-GCCCGCGGTGGAATGGACCCCCGCAAAGTGAGCG } \\
\text { AGC }\end{array}$ & $\underline{\text { EheI-SacII }}$ \\
\hline & $\mathrm{AB} 101 \mathrm{r}$ & $\begin{array}{l}\text { CCCAAGCTTATTCTTCCCTTTGGGCTTTTTCATGCTCTT } \\
\text { CTCG }\end{array}$ & $\underline{\text { HindIIII }}$ \\
\hline \multirow[t]{2}{*}{$\operatorname{Hip}(1-368)$} & AB99f & $\begin{array}{l}\text { phos-GCCCGCGGTGGAATGGACCCCCGCAAAGTGAGCG } \\
\text { AGC }\end{array}$ & $\underline{\text { EheI-SacII }}$ \\
\hline & $\mathrm{AB} 102 \mathrm{r}$ & $\begin{array}{l}\text { CCCAAGCTTATGAGTGACCTCCAAACTTGGCTGACAA } \\
\text { TTTACTG }\end{array}$ & HindIII \\
\hline \multirow[t]{2}{*}{$\operatorname{Hip}(45-267)$} & LZ_HIP5f & $\begin{array}{l}\text { CCCCCATGGCTACTCATAAAGCGAAGTCAGAAGAA A } \\
\text { AC }\end{array}$ & $\underline{\mathrm{NcoI}}$ \\
\hline & LZ02r & $\begin{array}{l}\text { CGGGGTACCTTATTCTTCCCTTTGGGCTTTTTCATGCT } \\
\text { CTTCTCG }\end{array}$ & $\underline{K p n I}$ \\
\hline \multirow[t]{2}{*}{ Hip(45-368) } & LZ_HIP5f & $\begin{array}{l}\text { CCCCCATGGCTACTCATAAAGCGAAGTCAGAAGAAA } \\
\text { AC }\end{array}$ & $\underline{N c o I}$ \\
\hline & $\mathrm{AB} 102 \mathrm{r}$ & $\begin{array}{l}\text { CCCAAGCTTATGAGTGACCTCCAAACTTGGCTGACAA } \\
\text { TTTACTG }\end{array}$ & $\underline{\text { Hind IIII }}$ \\
\hline \multirow[t]{2}{*}{$\operatorname{Hip}(78-234)$} & $\mathrm{AB} 100 \mathrm{f}$ & $\begin{array}{l}\text { phos-GCCCGCGGTGGAAGCGATCTAGAAAT } \\
\text { TGACAATGAAGGTGTAATTGAAGC }\end{array}$ & $\underline{\text { EheI-SacII }}$ \\
\hline & LZ_HIP4r & $\begin{array}{l}\text { CCCAAGCTTACCGATGTTCAGCAATTTTTTGAGCCCG } \\
\text { AGG }\end{array}$ & $\underline{\text { HindIIII }}$ \\
\hline \multirow[t]{2}{*}{ Hip(78-247) } & $\mathrm{AB} 100 \mathrm{f}$ & $\begin{array}{l}\text { phos-GCCCGCGGTGGAAGCGATCTAGAAAT } \\
\text { TGACAATGAAGGTGTAATTGAAGC }\end{array}$ & $\underline{\text { EheI-SacII }}$ \\
\hline & LZ20r & $\begin{array}{l}\text { CCCAAGCTTATTTTATCTCTCGCTCTTCACGTTTTCGCT } \\
\text { CATA }\end{array}$ & $\underline{\text { HindIII }}$ \\
\hline \multirow[t]{2}{*}{$\operatorname{Hip}(78-267)$} & AB100f & $\begin{array}{l}\text { phos-GCCCGCGGTGGAAGCGATCTAGAAAT } \\
\text { TGACAATGAAGGTGTAATTGAAGC }\end{array}$ & EheI-SacII \\
\hline & $\mathrm{AB} 101 \mathrm{r}$ & $\begin{array}{l}\text { CCCAAGCTTATTCTTCCCTTTGGGCTTTTTCATGCTCTT } \\
\text { CTCG }\end{array}$ & $\underline{\text { HindIIII }}$ \\
\hline \multirow[t]{2}{*}{$\operatorname{Hip}(78-368)$} & $\mathrm{AB} 100 \mathrm{f}$ & $\begin{array}{l}\text { phos-GCCCGCGGTGGAAGCGATCTAGAAAT } \\
\text { TGACAATGAAGGTGTAATTGAAGC }\end{array}$ & $\underline{E h e \mathrm{I}}-$ SacII \\
\hline & $\mathrm{AB} 102 \mathrm{r}$ & $\begin{array}{l}\text { CCCAAGCTTATGAGTGACCTCCAAACTTGGCTGACAA } \\
\text { TTTACTG }\end{array}$ & $\underline{\text { HindIIII }}$ \\
\hline \multirow[t]{2}{*}{$\operatorname{Hip}(107-267)$} & LZ23f & GCCGAGGCGATGATGGATGAAGCAAATGAAAAG & EheI \\
\hline & $\mathrm{AB} 101 \mathrm{r}$ & $\begin{array}{l}\text { CCCAAGCTTATTCTTCCCTTTGGGCTTTTTCATGCTCTT } \\
\text { CTCG }\end{array}$ & $\underline{\text { HindIIII }}$ \\
\hline \multirow[t]{2}{*}{$\operatorname{Hip}(312-368)$} & LZ_HIP3f & $\begin{array}{l}\text { CATGCCATGGGACTCAACGAAATCCTCAGTGACCCAG } \\
\text { AG }\end{array}$ & $\underline{N c o \mathrm{I}}$ \\
\hline & $\mathrm{AB} 102 \mathrm{r}$ & $\begin{array}{l}\text { CCCAAGCTTATGAGTGACCTCCAAACTTGGCTGACAA } \\
\text { TTTACTG }\end{array}$ & $\underline{\text { HindIII }}$ \\
\hline
\end{tabular}


Table 16: Primer utilized for generation of Hip constructs in this study.

The names of constructs, primers with respective nucleotide sequences and the corresponding restriction endonucleases sites are listed in the respective columns. The underlined residues indicate restriction endonuclease sites introduced for cloning. The 5'- phosphorylation of forward primers is indicated by phos.

\begin{tabular}{|c|c|c|}
\hline Hip Mutant & Primer & Sequence 5' $\rightarrow$ 3' \\
\hline \multirow[t]{2}{*}{ K117A } & LZ89f_K117A & ATGAAGCAAATGAAAAGGCGGGGGCTGCCATCGACGCTC \\
\hline & LZ90r_K117A & GAGCGTCGATGGCAGCCCCCGCCTTTTCATTTGCTTCAT \\
\hline \multirow[t]{2}{*}{ G118V } & LZ34f_HIPFM1 & GAAGCAAATGAAAAGAAGGTGGCTGCCATCGACGCTCTA \\
\hline & LZ35r_HIPFM1 & TAGAGCGTCGATGGCAGCCACCTTCTTTTCATTTGCTTC \\
\hline \multirow[t]{2}{*}{$\mathrm{I} 148 \mathrm{~A}$} & LZ93f_I148A & CTAAACCCTCGCTTGGCCGCCCTGTATGCCAAGAGA \\
\hline & LZ94r_I148A & TCTCTTGGCATACAGGGCGGCCAAGCGAGGGTTTAG \\
\hline \multirow[t]{2}{*}{ N177K } & LZ70f_N177K & GACAGAGCTATTGAAATAAAGCCTGATTCAGCTCAGCCA \\
\hline & LZ71r_N177K & TGGCTGAGCTGAATCAGGCTTTATTTCAATAGCTCTGTC \\
\hline \multirow[t]{2}{*}{ S180E } & LZ96f2_S180E & ATTGAAATAAACCCTGATGAGGCTCAGCCATACAAATGGAGA \\
\hline & LZ97r2_S180E & TCTCCATTTGTATGGCTGAGCCTCATCAGGGTTTATTTCAAT \\
\hline \multirow[t]{2}{*}{ K185E } & LZ72f_K185E & GATTCAGCTCAGCCATACGAATGGAGAGGGAAAGCGCAC \\
\hline & LZ73r_K185E & GTGCGCTTTCCCTCTCCATTCGTATGGCTGAGCTGAATC \\
\hline \multirow[t]{2}{*}{ L210S } & LZ87f_L210S & TTGCCCTGGCCTGTAAATCCGACTATGATGAGGACGC \\
\hline & LZ88r_L210S & GCGTCCTCATCATAGTCGGATTTACAGGCCAGGGCAA \\
\hline \multirow[t]{2}{*}{ N177A/D179A } & LZ85f_ND & AGAGCTATTGAAATAGCCCCTGCCTCAGCTCAGCCATAC \\
\hline & LZ86r_ND & GTATGGCTGAGCTGAGGCAGGGGCTATTTCAATAGCTCT \\
\hline \multirow[t]{2}{*}{ H196S/E199A } & LZ91f_HE & ACAGACTCCTGGGTTCTTGGGAAGCCGCAGCTCGCGAT \\
\hline & LZ92r_HE & ATCGCGAGCTGCGGCTTCCCAAGAACCCAGGAGTCTGT \\
\hline \multirow[t]{2}{*}{ D211K_Y212A } & LZ74f_2M & CTGGCCTGTAAATTGAAGGCCGATGAGGACGCCAGTGCA \\
\hline & LZ75r_2M & TGCACTGGCGTCCTCATCGGCCTTCAATTTACAGGCCAG \\
\hline \multirow[t]{2}{*}{$\mathrm{R} 235 \mathrm{~A} / \mathrm{K} 236 \mathrm{D}$} & LZ83f_RK & $\begin{array}{l}\text { AAATTGCTGAACATCGGGCCGACTATGAGCGAAAACGTGAAG } \\
\text { AGC }\end{array}$ \\
\hline & LZ84r_RK & $\begin{array}{l}\text { GCTCTTCACGTTTTCGCTCATAGTCGGCCCGATGTTCAGCAAT } \\
\text { TT }\end{array}$ \\
\hline \multirow[t]{2}{*}{$\mathrm{S} 74 \mathrm{E} / \mathrm{S} 75 \mathrm{E} / \mathrm{S} 78 \mathrm{E}$} & LZ95f_3E & $\begin{array}{l}\text { GCCGGAGAGGAGGAGGAGGAGGATCTAGAAATTGACAATGA } \\
\text { AGGTGTAATTGAA }\end{array}$ \\
\hline & LZ20r & CCCAAGCTTATTTTATCTCTCGCTCTTCACGTTTTCGCT CATA \\
\hline \multirow[t]{2}{*}{ S74E/S75E/S78E } & LZ100f_3E & $\begin{array}{l}\text { AAGACAGAGGAGCCAGAGGAGGAGGAGGAGGATCTAGAAA } \\
\text { TT }\end{array}$ \\
\hline & LZ101r_3E & AATTTCTAGATCCTCCTCCTCCTCCTCTGGCTCCTCTGTCTT \\
\hline \multirow[t]{2}{*}{ S74N/S75N/S78N } & LZ98f_3N & $\begin{array}{l}\text { AAGACAGAGGAGCCAAACAACGAGGAGAACGATCTAGAAAT } \\
\text { TGAC }\end{array}$ \\
\hline & LZ99r_3N & $\begin{array}{l}\text { GTCAATTTCTAGATCGTTCTCCTCGTTGTTTGGCTCCTCTGTCT } \\
\mathrm{T}\end{array}$ \\
\hline
\end{tabular}


Table 17: Primers utilized for site-directed mutagenesis on Hip.

Point mutations were introduced via a PCR based mutagenesis strategy into Hip. The sites of point mutations are listed on the left column. The respective names of the primer and the corresponding oligonucleotide sequences are listed. The modified sites are highlighted by bold characters.

\begin{tabular}{|c|c|c|c|}
\hline Construct & Primer & Sequence $5^{\prime} \rightarrow 3^{\prime}$ & Endonuclease \\
\hline \multirow[t]{3}{*}{$\begin{array}{l}\text { Hip(78-234)-3aa- } \\
\text { Hsp70N }\end{array}$} & $\mathrm{AB} 100 \mathrm{f}$ & $\begin{array}{l}\text { phos-GCCCGCGGTGGAAGCGATCTAGAAAT } \\
\text { TGACAATGAAGGTGTAATTGAAGC }\end{array}$ & $\underline{\text { EheI-SacII }}$ \\
\hline & LZ43r & $\begin{array}{l}\text { CCCGGATCCACCCCGATGTTCAGCAATTTTT } \\
\text { TGAGCCCGAGG }\end{array}$ & $\underline{B a m H I}$ \\
\hline & LZ51r & 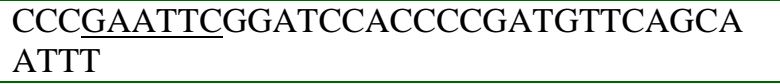 & $\underline{E c o R I}$ \\
\hline \multirow[t]{3}{*}{$\begin{array}{l}\text { Hip(78-234)-6aa- } \\
\text { Hsp70N }\end{array}$} & $\mathrm{AB} 100 \mathrm{f}$ & $\begin{array}{l}\text { phos-GCCCGCGGTGGAAGCGATCTAGAAAT } \\
\text { TGACAATGAAGGTGTAATTGAAGC }\end{array}$ & $\underline{E h e I-S a c I I}$ \\
\hline & LZ44r & $\begin{array}{l}\text { CCCGGATCCACCGGAGCCACCCCGATGTTCA } \\
\text { GCAATTTTTTGAGCCCG }\end{array}$ & $\underline{B a m H I}$ \\
\hline & LZ52r & CCGAATTCGGATCCACCGGAGCCACCCCG & $\underline{E c o R I}$ \\
\hline \multirow[t]{4}{*}{$\begin{array}{l}\text { Hip(78-234)-9aa- } \\
\text { Hsp70N }\end{array}$} & AB100f & $\begin{array}{l}\text { phos-GCCCGCGGTGGAAGCGATCTAGAAAT } \\
\text { TGACAATGAAGGTGTAATTGAAGC }\end{array}$ & $\underline{E h e \mathrm{I}}-\mathrm{SacII}$ \\
\hline & LZ45r & $\begin{array}{l}\text { GGAGCCACCTCCAGCACCCCGATGTTCAGCA } \\
\text { ATTTTTTGAGCCCG }\end{array}$ & - \\
\hline & LZ46r & $\begin{array}{l}\text { CCCGGATCCACCGGAGCCACCTCCAGCACCC } \\
\text { CG }\end{array}$ & $\underline{B a m H I}$ \\
\hline & LZ53r & CCCGAATTCGGATCCACCGGAGCCACCTCC & $\underline{E c o R I}$ \\
\hline \multirow[t]{2}{*}{ HipM-3aa-Hsp70N } & AB100f & $\begin{array}{l}\text { phos-GCCCGCGGTGGAAGCGATCTAGAAAT } \\
\text { TGACAATGAAGGTGTAATTGAAGC }\end{array}$ & $\underline{E h e I-S a c I I}$ \\
\hline & LZ76r_F3aa & $\begin{array}{l}\text { CCCGGATCCACCTTTTATCTCTCGCTCTTCAC- } \\
\text { GTTTTCGCTCA }\end{array}$ & $\underline{B a m H I}$ \\
\hline \multirow[t]{2}{*}{ HipM-6aa-Hsp70N } & $\mathrm{AB} 100 \mathrm{f}$ & $\begin{array}{l}\text { phos-GCCCGCGGTGGAAGCGATCTAGAAAT } \\
\text { TGACAATGAAGGTGTAATTGAAGC }\end{array}$ & $\underline{E h e \mathrm{I}}-\mathrm{SacII}$ \\
\hline & LZ77r_F6aa & $\begin{array}{l}\text { CCCGGATCCACCGGAGCCACCTTTTATCTCTC } \\
\text { GCTCTTCACGTTTTCGCTCA }\end{array}$ & $\underline{B a m H I}$ \\
\hline \multirow[t]{3}{*}{ HipM-9aa-Hsp70N } & $\mathrm{AB} 100 \mathrm{f}$ & $\begin{array}{l}\text { phos-GCCCGCGGTGGAAGCGATCTAGAAAT } \\
\text { TGACAATGAAGGTGTAATTGAAGC }\end{array}$ & $\underline{\text { EheI-SacII }}$ \\
\hline & LZ78r_F9aa & $\begin{array}{l}\text { GGAGCCACCTCCAGCACCTTTTATCTCTCGCT } \\
\text { CTTCACGTTTTCGCTCA }\end{array}$ & - \\
\hline & LZ79r_F9aa & $\begin{array}{l}\text { CCCGGATCCACCGGAGCCACCTCCAGCACCTT } \\
\text { TTAT }\end{array}$ & $\underline{B a m H I}$ \\
\hline \multirow[t]{4}{*}{ Hsp70N-HipM } & LZ80f_Hsp70N & CCCGAATTCATGGCCAAAGCCGCGGCGATCG & $\underline{E c o R I}$ \\
\hline & LZ81r_Hsp70N & CCCTCGAGCCCCATCAGGATGGCCGCCTG & $\underline{X h o I}$ \\
\hline & LZ82f_HIPF & $\begin{array}{l}\text { GGGCTCGAGAGCGATCTAGAAATTGACAATG } \\
\text { AAGGTGTAATTGAA }\end{array}$ & $\underline{X h o I}$ \\
\hline & LZ20r & $\begin{array}{l}\text { CCCAAGCTTATTTTATCTCTCGCTCTTCACGTT } \\
\text { TTCGCTCATA }\end{array}$ & $\underline{\text { HindIII }}$ \\
\hline
\end{tabular}

Table 18: Primers utilized for generation of Hip-Hsp70 fusion protein in this study.

The names of fusion proteins, primers with respective nucleotide sequences and the corresponding restriction endonuclease sites are listed in columns. The underlined residues indicate the restriction sites introduced for cloning. The phosphorylation of forward primers is indicated by phos. 


\begin{tabular}{|c|c|c|c|}
\hline Construct & Primer & Sequence 5' $\rightarrow$ 3' & Endonuclease \\
\hline \multirow[t]{2}{*}{ Hip(1-267) } & LZ102f & $\begin{array}{l}\text { phos-GCCACTAGTATGGATCCCCGCAAAGTGAG } \\
\text { CGAG }\end{array}$ & $\begin{array}{l}\text { EheI-SpeI-ATG } \\
\text { start codon }\end{array}$ \\
\hline & LZ110r & $\begin{array}{l}\text { CCGAATTCTTATTCTTCCCTTTGGGCTTTTTCAT } \\
\text { GCTCTTC TCG }\end{array}$ & $\underline{E c o R I}$ \\
\hline \multirow[t]{2}{*}{ Hip(1-368) } & LZ102f & $\begin{array}{l}\text { phos-GCCACTAGTATGGATCCCCGCAAAGTGAGC } \\
\text { GAG }\end{array}$ & $\begin{array}{l}\text { EheI-SpeI-ATG } \\
\text { start codon }\end{array}$ \\
\hline & LZ109r & $\begin{array}{l}\text { CCGAATTCTTATGAGTGACCTCCAAACTTGGCTG } \\
\text { ACAATTTACTG }\end{array}$ & $\underline{E c o R I}$ \\
\hline \multirow[t]{2}{*}{ Hip(45-368) } & LZ103f & $\begin{array}{l}\text { phos-GCCACTAGTATGGCTACTCATAAAGCGAAG } \\
\text { TCAGAAGAAAACAC }\end{array}$ & $\begin{array}{l}\text { EheI-SpeI-ATG } \\
\text { start codon }\end{array}$ \\
\hline & LZ109r & $\begin{array}{l}\text { CCGAATTCTTATGAGTGACCTCCAAACTTGGCTG } \\
\text { ACAATTTACTG }\end{array}$ & $\underline{E c o R I}$ \\
\hline \multirow[t]{2}{*}{$\begin{array}{l}\text { pProEx-HtB } \\
\text { (SpeI site mutant) }\end{array}$} & $\begin{array}{l}\text { LZ111f_SpeI } \\
\text { mut }\end{array}$ & $\begin{array}{l}\text { CTACGTCGACGAGCTCTATAGTCGCGGCCGCTT } \\
\text { TCGAA }\end{array}$ & - \\
\hline & $\begin{array}{l}\text { LZ112r_SpeI } \\
\text { mut }\end{array}$ & $\begin{array}{l}\text { TTCGAAAGCGGCCGCGACTATAGAGCTCGTCGA } \\
\text { CGTAG }\end{array}$ & - \\
\hline
\end{tabular}

Table 19: Primers utilized for generation of Hip constructs for the in vivo studies in yeast.

The names of constructs, primers with respective nucleotides and the corresponding restriction endonucleases are listed in the respective column. The underlined residues indicate restriction endonuclease sites introduced for cloning purposes. The phosphorylation of forward primers is indicated by phos. Since the yeast vectors p423GPD or p423ADP contains no start codon, ATG start codons were introduced via PCR amplification. ATG start codon and mutated SpeI site in pProEx-HtB vector are also highlighted in bold. The Hip mutants for yeast study were generated using the respective primers in Table 17.

\begin{tabular}{lll}
\hline Hsp70 Mutant & Primer & Sequence $\mathbf{5}^{\prime} \rightarrow \mathbf{3}$ \\
\hline Hsp70_D199A & LZ120f_D199A & AACGTGCTCATCTTTGCCCTGGGCGGGGGCACCT \\
\hline & LZ121r_D199A & AGGTGCCCCCGCCCAGGGCAAAGATGAGCACGTT \\
\hline Hsp70_T204A & T204A_f & TTTGACCTGGGCGGGGGCGCGTTCGACGTGTCCAT \\
\hline & T204A_r & ATGGACACGTCGAACGCGCCCCCGCCCAGGTCAAA \\
\hline
\end{tabular}

Table 20: Primers utilized for site-directed mutagenesis on full length human Hsp70.

Point mutants of Hsp70variants were investigated via a PCR based mutagenesis strategy. The sites of point mutants are listed on the left column. The names of primer and the corresponding nucleotides are listed, respectively. The mutated sites are highlighted in bold. 


\subsection{Hip alignment}

R_norvegicus T-adhaerens S-mansoni D-melanogaster Ehistolytica T-brucei

L_braziliensis Olucimarinus T-gondii P-falciparum A thaliana consensus $>50$ contacts to Hsp70 post-transl. modif. mutation sites

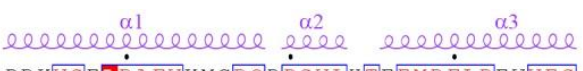

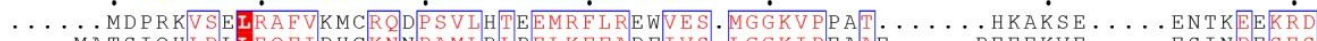

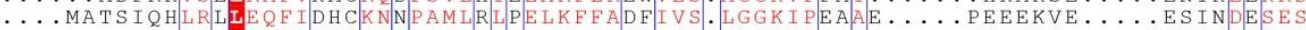

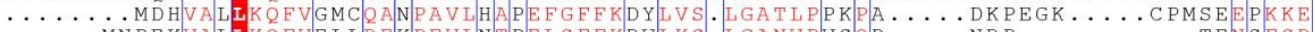

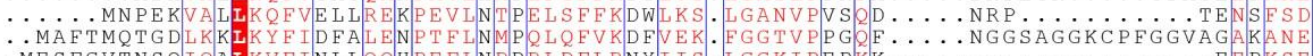

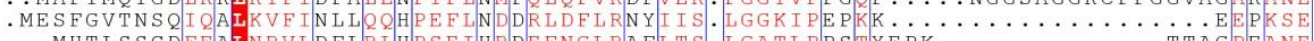

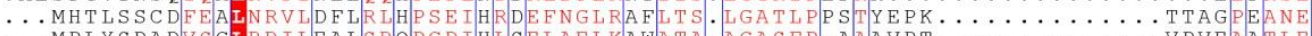

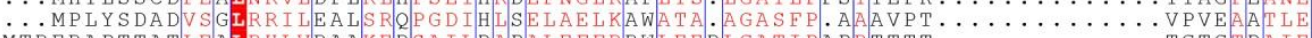
MTRERAPTTATLEALRLVRAAKEDSA I DAPALEFERRLEEDLGATIPAPRTTTT $\ldots \ldots \ldots \ldots \ldots \ldots$

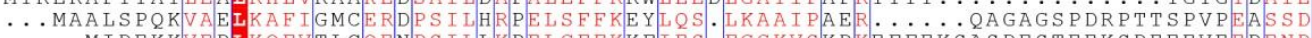
$\ldots . . . M I D E K K V E D L K Q F V T L C Q N$ PS I L KPELGFFKKFIES. FGGKVSKDKEFFEKSASDESTEEKSDEEEVEEDEND

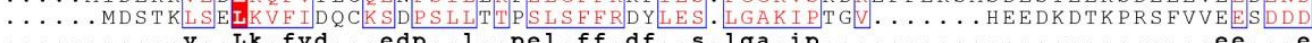
0
0

$\begin{array}{ll}\text { R_norvegicus } & 62 \\ \text { T-adhaerens } & 66 \\ \text { C-clegans } & 62 \\ \text { S-mansoni } & 58 \\ \text { D-melanogaster } & 73 \\ \text { E-histolytica } & 59 \\ \text { T-brucei } & 63 \\ \text { D-braziliensis } & 62 \\ \text { O-lucimarinus } & 67 \\ \text { T_gondii } & 71 \\ \text { P-falciparum } & 75 \\ \text { A-thaliana } & 67 \\ \text { consensus }>50 & \\ \text { contacts to Hsp70 } \\ \text { post-transl. modif. }\end{array}$

mutation sit. modif.

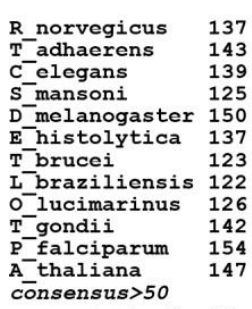

contacts to Hsp70

post-transl. modif.

mutation sites

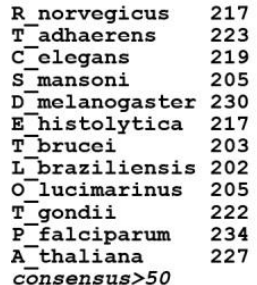

- -

contacts to Hsp70 post-transl. modif. mutation sites eleereeeele

a.4 TIEN..... QVGSAN..... DSGADETS...ES.......ETEF.... DDEVILPE PVPDLAMGDDSTEVT DEMREKAEEKKCEAMAKMSDGDLTGAVDLE

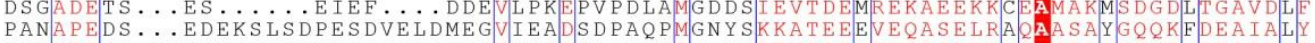

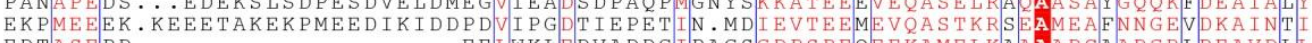

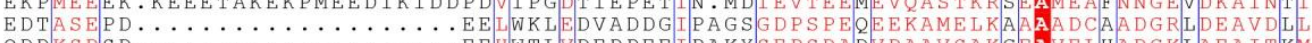
Q D DKS DSD . . . . . . . . .

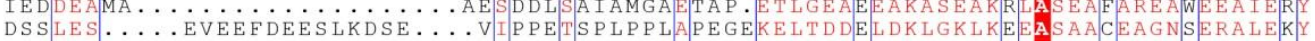

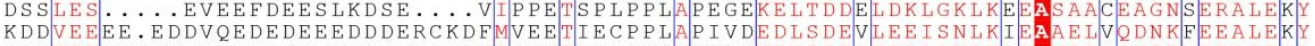
MDETEEVKPKVEEEEEEDEIVESDVELEGDTVED N PQKMGDS $\ldots$ ec...e..........e.vie.d..pq...d..e.teee.e.a.e.k i

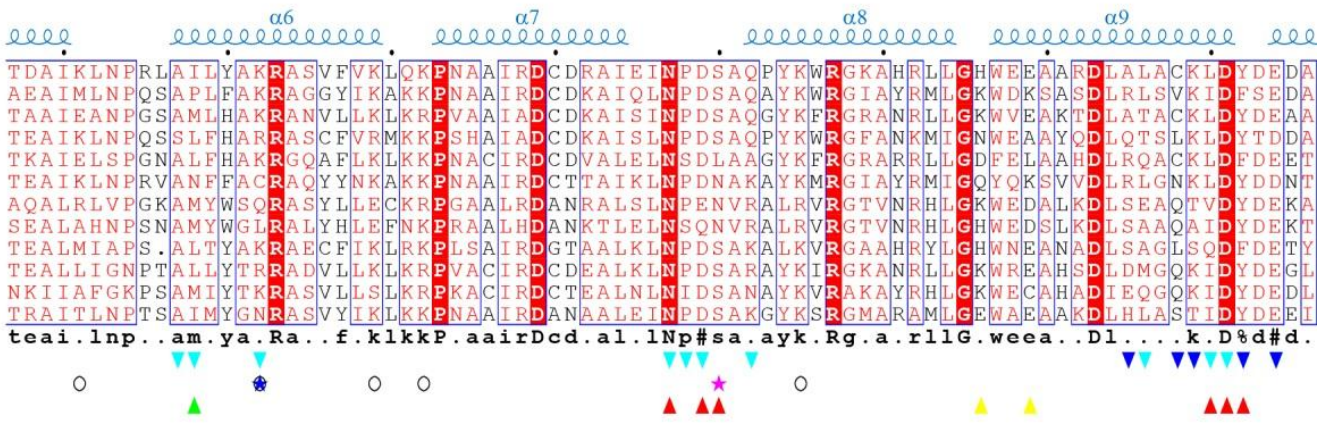

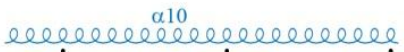

SAMLREVQPRA

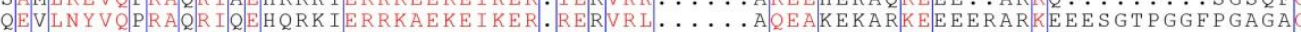

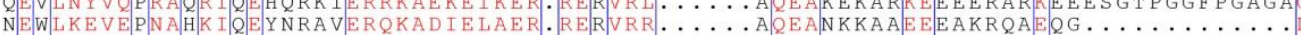

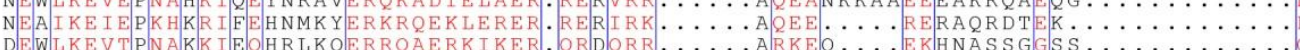

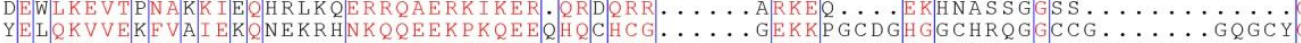

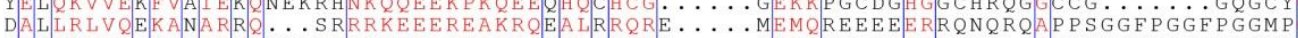
NETLKFVOFRAMORHKRELAROHAEEAAVARROEELRRORO ......REAAEEAAATAEAOAASGGMPGGMPGGFPGGMP

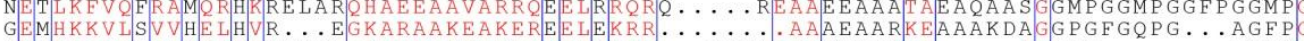

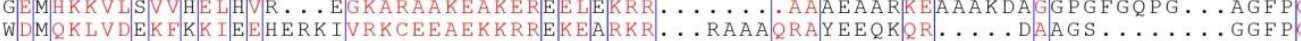
WEMOKLIEEKYKKIYEKRRFKINREEEKKRKKREKELKKRLAARKAAEKAYKANNKRENYDSDSSDSSYSEPDESGDFP

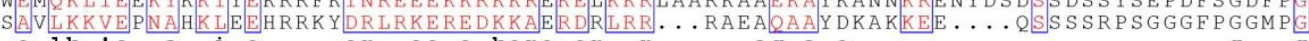

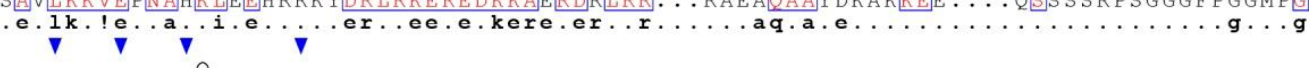
$\mathbf{\Delta} \mathbf{\Delta}$

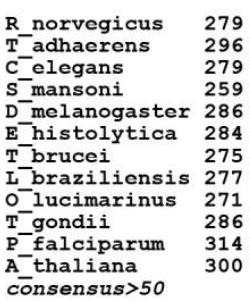

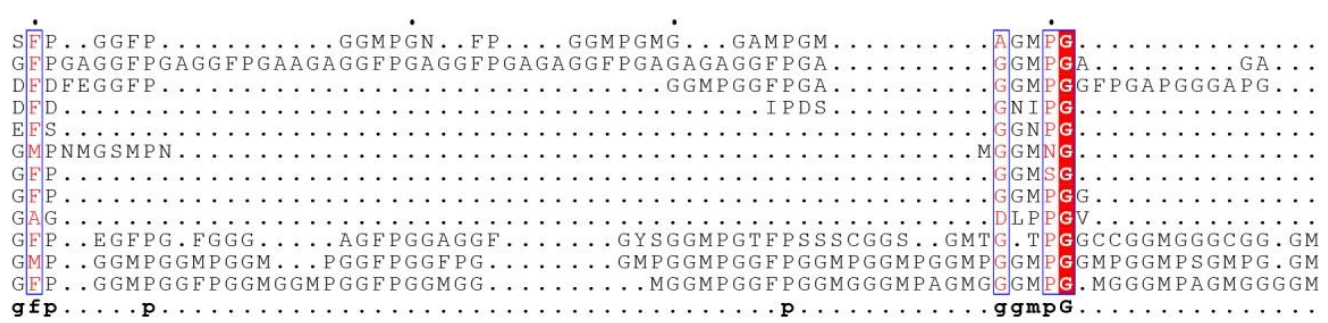



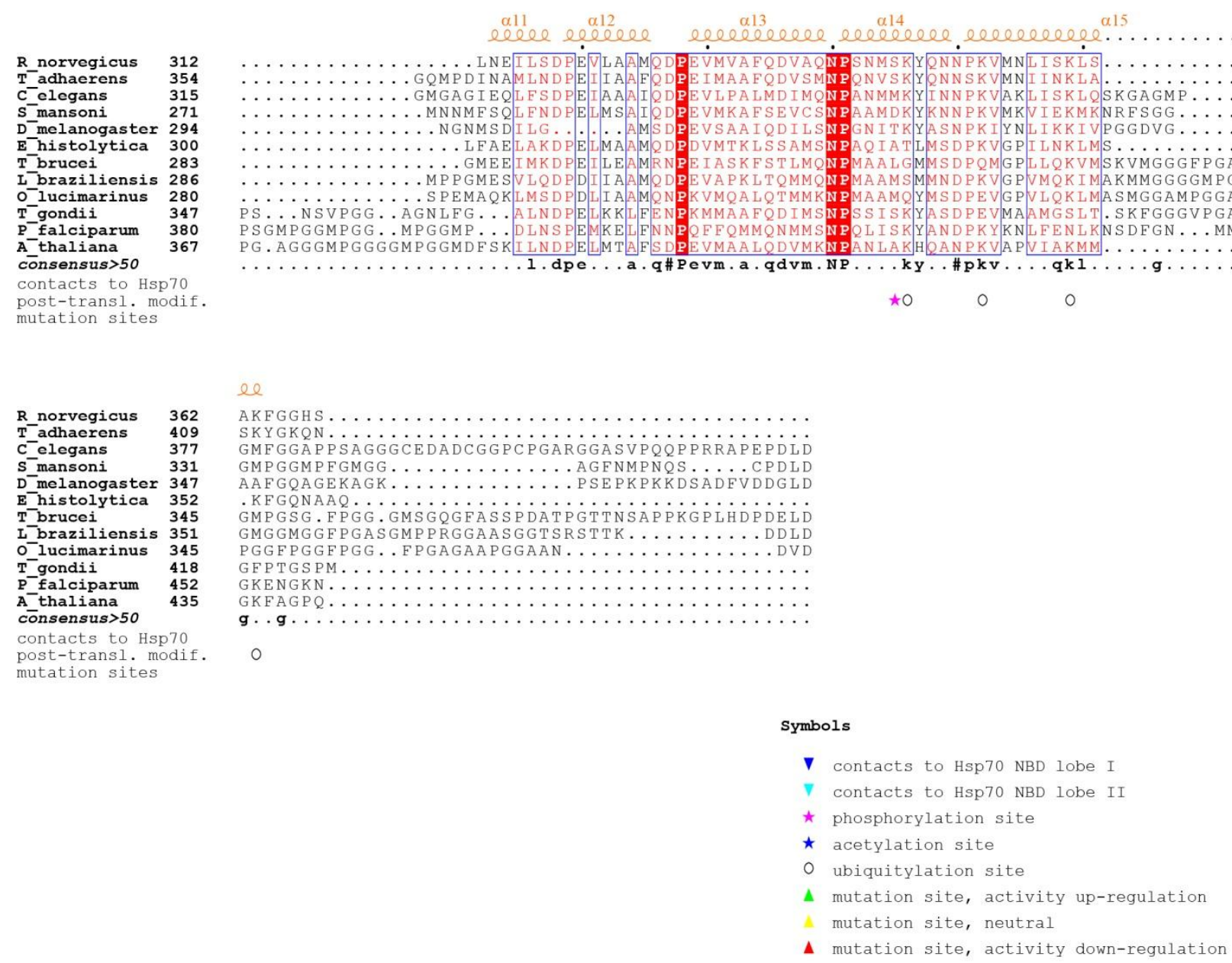

Figure 45: Alignment of Hip sequences.

Amino acid sequences of selected Hip homologs were aligned using the EBI Clustal-X server. Secondary structure elements for Hip from Rattus norvegicus are indicated above the sequences. The Hip domain structure is indicated by purple, blue and orange coloring of secondary structure elements in the dimerization, TPR and DP domains, respectively. Similar residues are shown in red and identical residues in white on a red background. Blue frames indicate homologous regions. The consensus sequence is shown at the bottom. Downward pointing arrowheads indicate interface residues facing the NBD of Hsp70. Blue and cyan colors indicate contacts to subdomains IA/IB and IIB of the Hsp70 NBD, respectively. Known acetylation and phosphorylation sites are indicated by asterisks in dark blue and purple, respectively. Reported ubiquitylation sites are shown as hollow circles. Mutation sites are indicated by upward pointing arrowheads, and colored according to their effect on Hsp70 binding. Green, yellow and red colors indicate decreased, neutral and increased dissociation rates of MABA-ADP from Hsp70N. The Uniprot/TREMBL accession codes for the sequences are: P50503, Rattus norvegicus; B3RY90, Trichoplax adhaerens; G5EE04, Caenorhabditis elegans; G4VJJ1, Schistosoma mansoni; Q86DS1, Drosophila melanogaster; C4M2C4, Entamoeba histolytica; C9ZKP5, Trypanosoma brucei gambiense (strain MHOM/CI/86/DAL972); A4HH33, Leishmania braziliensis; A4S4D2, Ostreococcus lucimarinus (strain CCE9901); B9Q4N1, Toxoplasma gondii; Q8I3J0, Plasmodium falciparum (isolate 3D7); Q93YR3, Arabidopsis thaliana. 


\subsection{Abbreviations}

\begin{tabular}{ll}
\hline AD & Alzheimer's Disease \\
ADP & Adenosine 5'-diphosphate \\
AIP & Aryl hydrocarbon receptor interacting protein \\
AMP & Ampicillin \\
APS & Ammonium peroxydisulfate \\
AR & Androgen receptor \\
ATP & Adenosine 5'-triphosphate \\
BAG & Bcl2-associated athanogene \\
BLAST & Basic Local Alignment Search Tool \\
bp & base pairs \\
BSA & Bovine serum albumin \\
CD & Circular dichroism \\
CD & C-terminal domain \\
CFTR & Cystic-fibrosis transmembrane-conductance regulator \\
CHIP & C-terminus of Hsp70-interacting protein \\
CIP & Calf intestinal phosphatase \\
CV & Column volume \\
CXCR2 & C-X-C-motif chemokine receptors type 2 \\
CXCR4 & C-X-C-motif chemokine receptors type 4 \\
DNA & Deoxyribonucleic acid \\
DnaJ & Bacterial Hsp40 chaperone \\
DnaK & Bacterial Hsp70 chaperone \\
dNTPs & Deoxyribonucleoside triphosphates \\
DOC & Deoxycorticosterone \\
DTT & Dithiothreitol \\
ECL & enhanced chemiluminescence \\
E.coli & Escherichia coli \\
EDTA & Ethylenediaminetetraacetic acid \\
ER & \\
\hline &
\end{tabular}




\begin{tabular}{ll}
\hline ESRF & European Synchrotron Radiation Facility \\
FKBP & FK506-binding protein \\
GR & Glucocorticoid Receptor \\
GRE & Glucocorticoid Responsive Element \\
GRK5 & G protein-coupled receptor Kinase 5 \\
GroEL & Large growth E gene product \\
GroES & Small growth E gene product \\
GrpE & Growth P-like gene E \\
HEPES & N-(2-hydroxyethyl)piperazin-N'-2-ethanesulfonic acid \\
Hip & Hsp70-interacting protein \\
His 6 & Hexa-histidine tagged \\
Hsf1 & Heat shock factor 1 \\
Hop & Hsp70-Hsp90 organizing protein \\
HPD & histidine-proline-aspartate \\
Htt & Huntingtin \\
Hsp & Heat shock protein \\
HspBP1 & Hsp70 binding protein 1 \\
IMAC & Immobilised metal ion affinity chromatography \\
IPTG & Isopropyl- $\beta$-D-1 thiogalactopyranoside \\
ITC & Isothermal titration calorimetry \\
Kan & Kanamycin \\
LB & Luria Bertani medium \\
MD & Middle domain \\
MOPS & 3-(N-morpholino)propanesulfonic acid \\
MW & Molecular weight \\
NAC & Nascent chain-associated complex \\
NBD & Nucleotide binding domain \\
ND & N-terminal domain \\
NEF & \\
NMR & Noteotide exchange factor \\
\hline &
\end{tabular}




\begin{tabular}{ll}
\hline PCR & Polymerase chain reaction \\
PDB & Protein data bank \\
PEG & Polyethylene glycol \\
Pfu & Pyrococcus furiosus \\
PGK & Phosphoglycerate kinase \\
PMSF & Phenylmethylsulfonylfluoride \\
PP5 & Phosphoprotein phosphatase 5 \\
RAC & Ribosome-associated complex \\
rpm & revolutions per minute \\
RT & Room temperature \\
SBD & Substrate binding domain \\
SC & Synthetic complete \\
S.cerevisiae & Saccharomyces cerevisiae \\
SDS-PAGE & Sodiumdodecylsulfate polyacrylamide geleletrophoresis \\
sHsp & Small heat shock protein \\
SIRAS & Single isomorphous replacement with anomalous scattering \\
SLS & Swiss Synchrotron Light Source \\
SOD & Superoxide dismutase 1 \\
TEM & Transmission Electron microscopy \\
TEMED & N, N, N', N'-tetramethylethlylenediamine \\
TEV & Tobacco etch virus \\
TF & Trigger factor \\
TPR & Tetratricopeptide repeat \\
TRiC & TCP-1 ring complex \\
Tris & Tris(hydroxymethyl)aminomethane \\
UPS & Ubiquitin Proteasome System \\
UV & Ultra violet \\
w/v & Volts \\
\hline &
\end{tabular}




\title{
8.4 Curriculum Vitae
}

\author{
Name: $\quad$ Zhuo Li \\ Date of birth: $\quad$ 05.April.1980 \\ Birth of place: Dalian, Liaoning, China \\ Citizenship: Chinese \\ EDUCATION \\ 11/2009 - present \\ 05/2007-10/2009 \\ PhD Dissertation \\ Max Planck Institute of Biochemistry, Martinsried, Germany \\ Cellular Biochemistry Department of Prof. Dr. F. U. Hartl \\ Title: "Structure and Function of Hip, an Attenuator of the Hsp70 \\ Chaperone Cycle" \\ Master of Science (M. Sc.) \\ Ludwig-Maximilians-University Munich (LMU), Germany \\ Master thesis in the Cellular Biochemistry Department of Prof. Dr. \\ F. U. Hartl, Max Planck Institute of Biochemistry, Martinsried \\ Title: „Structural and Functional Characterization of the Hsp70 \\ Co-chaperone Hip" \\ $09 / 2003-04 / 2007$ \\ Bachelor of Science (B. Sc.) \\ Ludwig-Maximilians-University Munich (LMU), Germany \\ Bachelor thesis in the lab Prof. Dr. Paul Knochel at LMU \\ Title: „Towards a New Method for Palladium-catalyzed $N$-arylation“ \\ $09 / 1998-07 / 2002$ \\ Bachelor of Art (B. A.) \\ Liaoning University, Shenyang, China

\section{PUBLICATIONS} \\ Zhuo Li, F. Ulrich Hartl and Andreas Bracher \\ Structure and function of Hip, an attenuator of the Hsp70 chaperone \\ Nature Structural \& Molecular Biology, 20, 929-935 (2013) \\ Hongjun Ren, Zhuo Li and Paul Knochel \\ Chemoselective $\mathrm{C}\left(\mathrm{sp}^{3}\right)$-H-Bond Activation for the Preparation of \\ Condensed $N$-Heterocycles \\ Chem. Asian J. 2007, 2, 416-433.
}

